\title{
Novel Perspectives in Regulation of Chondrogenic Differentiation
}

Citation for published version (APA):

Caron, M. M. J. (2013). Novel Perspectives in Regulation of Chondrogenic Differentiation. [Doctoral Thesis, Maastricht University]. Maastricht University. https://doi.org/10.26481/dis.20130308mc

Document status and date:

Published: 01/01/2013

DOI:

10.26481/dis.20130308mc

Document Version:

Publisher's PDF, also known as Version of record

\section{Please check the document version of this publication:}

- A submitted manuscript is the version of the article upon submission and before peer-review. There can be important differences between the submitted version and the official published version of record.

People interested in the research are advised to contact the author for the final version of the publication, or visit the DOI to the publisher's website.

- The final author version and the galley proof are versions of the publication after peer review.

- The final published version features the final layout of the paper including the volume, issue and page numbers.

Link to publication

\footnotetext{
General rights rights.

- You may freely distribute the URL identifying the publication in the public portal. please follow below link for the End User Agreement:

www.umlib.nl/taverne-license

Take down policy

If you believe that this document breaches copyright please contact us at:

repository@maastrichtuniversity.nl

providing details and we will investigate your claim.
}

Copyright and moral rights for the publications made accessible in the public portal are retained by the authors and/or other copyright owners and it is a condition of accessing publications that users recognise and abide by the legal requirements associated with these

- Users may download and print one copy of any publication from the public portal for the purpose of private study or research.

- You may not further distribute the material or use it for any profit-making activity or commercial gain

If the publication is distributed under the terms of Article $25 \mathrm{fa}$ of the Dutch Copyright Act, indicated by the "Taverne" license above, 
Novel Perspectives in Regulation of Chondrogenic Differentiation 
Cover design: $\quad$ Lindi Bronneberg

Copyright: $\quad$ C Marjolein M.J. Caron, 2013

ISBN: $\quad 978-94-6191-616-7$

Printed by: $\quad$ Ipskamp Drukkers, Enschede, the Netherlands

Sponsors: $\quad$ Reumafonds, Annafonds, Garage Caron 


\section{Novel Perspectives in Regulation of Chondrogenic Differentiation}

\section{PROEFSCHRIFT}

ter verkrijging van de graad van doctor aan de Universiteit Maastricht, op gezag van de Rector Magnificus, Prof dr. L.L.G. Soete volgens het besluit van het College van Decanen, in het openbaar te verdedigen op vrijdag 08 maart 2013 om 12.00 uur door

Marjolein Maria Johanna Caron 
Promotor

Prof. dr. L.W. van Rhijn

\section{Copromotores}

Dr. T.J.M. Welting

Dr. P.J. Emans

Beoordelingscommissie

Prof. dr. ir. L.H. Koole (voorzitter)

Dr. F.A.van Nieuwenhoven

Prof. dr. G.J.V.M. van Osch (Erasmus University Medical Center Rotterdam, Nederland)

Dr. M. Poeze

Prof. dr. B. Zabel (Universitätsklinikum Freiburg, Freiburg, Deutschland)

The research presented in this thesis was conducted at the School for Public Health and Primary Care:

CAPHRI, Department Orthopaedic Surgery, of Maastricht University. CAPHRI participates in the Netherlands School of Primary Care Research CaRe. CAPHRI was classified as 'excellent' by the external evaluation committee of leading international experts that reviewed CAPHRI in December 2010.

This research was supported by the Dutch Arthritis Assiciation and the Dutch Anna Fonds 


\section{Table of Contents}

Chapter 1 General Introduction and Thesis Outline

Chapter 2 Activation of NF-кB Facilitates Early Chondrogenic Differentiation 39 during Endochondral Ossification

PloS ONE. 2012; 7(3): e33467

Chapter 3 Osmolarity Determines the in vitro Chondrogenic Differentiation

Capacity of Progenitor Cells via Nuclear Factor of Activated T-cells 5

Bone 2013; 53(1):p. 94-102

Chapter 4 Hypertrophic Differentiation During Chondrogenic Differentiation of Progenitor Cells is Stimulated by BMP-2 but Suppressed by BMP-7

Osteoarthritis and Cartilage 2013; In press

Chapter 5 Inhibition of Cyclooxygenase-2 Decreases Chondrocyte Hypertrophy in Endochondral Ossification

European Cells and Materials. 2011; 22: p.420-436

Chapter 6 Prostaglandins and COX-enzymes in Chondrogenic Differentiation of Progenitor Cells

In preparation

Chapter 7 Redifferentiation of Dedifferentiated Human Articular Chondrocytes: Comparison of 2D and 3D Cultures

Osteoarthritis and Cartilage. 2012; 20: p. 1170-1178

Chapter 8 General Discussion

Summary

Samenvatting

Dankwoord

Curriculum Vitae

List of Publications 



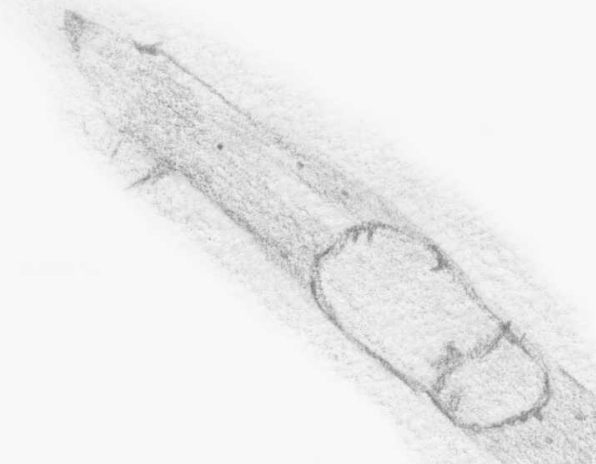

Chapter 1

\section{General Introduction and Thesis $O_{u t l i n e}$}

\section{Adapted from:}

Targeting Inflammatory Processes for Optimization of Cartilage Homeostasis and Repair Techniques Marjolein M.J. Caron, Tim J.M. Welting, Lodewijk W. van Rhijn and Pieter J. Emans

Department of Orthopaedic Surgery, Maastricht University Medical Center, Maastricht, the Netherlands

In: ICRS: Developing Insights in Cartilage Repair

Edited by: Prof. L. Peterson, P.J. Emans, Springer-Verlag London (Accepted for publication)

Endochondral Bone Formation as Blueprint for Regenerative Medicine

Pieter J. Emans, Marjolein M.J. Caron, Lodewijk W. van Rhijn and Tim. J.M. Welting

Department of Orthopaedic Surgery, Maastricht University Medical Center, Maastricht, the Netherlands

In: Tissue Regeneration - From Basic Biology to Clinical Application (2012)

Edited by: Prof. J. Davies, ISBN: 978-953-51-0387-5, InTech 



\section{Cartilage}

During our life moving, walking, sport, etc., are essential for our health and quality of life. Both bones and cartilage enable us to do so. Bones support us, allow muscles to move them, and protect vital internal organs. At their ends, most bones are covered with articular cartilage and form joints. Cartilage is not only present in articulating joints, but also in the rib-cage, ear, nose, bronchial tubes and intervertebral discs. It is essential for functions as breathing, hearing, articulation and locomotion. Based on its ultra-structural morphology and composition cartilage can be divided into three subtypes: hyaline-, elastic- and fibrocartilage. The most abundant of these is hyaline cartilage, which is present in a $2-4 \mathrm{~mm}$ layer on the articulating surfaces of long bones (Figure 1.1A). This hyaline articular cartilage is a truly remarkable material both structurally and functionally ${ }^{1-4}$. Its principal function is to provide a smooth, lubricated surface for articulation and is able to withstand an enormous amount of intensive and repetitive forces combined with low friction. The extracellular matrix (ECM) of hyaline articular cartilage determines these cartilage-specific properties. The hyaline cartilage ECM is mainly composed of water (65-80\%), collagens $(12-21 \%)$, proteoglycans $(6-10 \%)$ and other glycoproteins $(2-3.5 \%)^{5}$. Only $1-5 \%$ of the articular cartilage volume consists of chondrocytes, the main cell type found in articular cartilage $^{6}$ (Figure 1.1B). Typically, cartilage is devoid of blood vessels, lymphatics and nerve fibers. This implicates that cartilage is mainly hypoxic and chondrocytes receive their nutrients and oxygen via diffusion from the synovial fluid, through the surrounding extracellular matrix and from the underlying subchondral bone ${ }^{7}$.
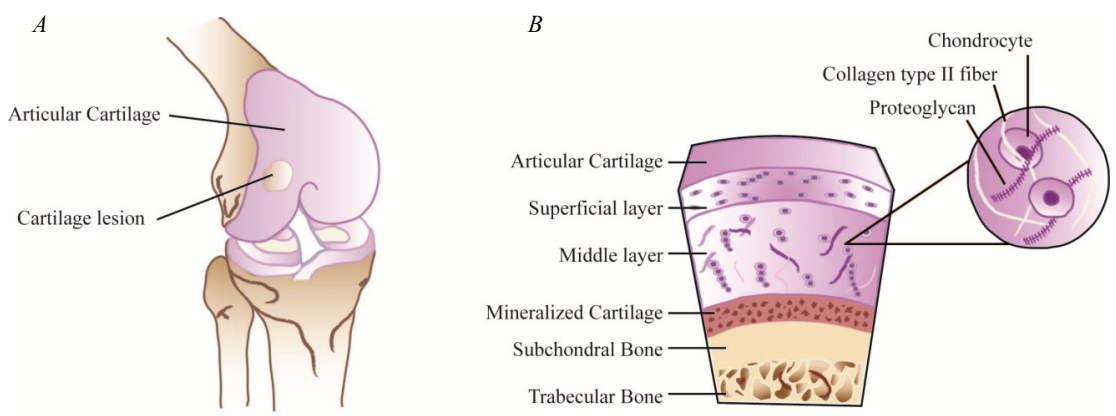

Figure 1.1: Schematic representation of a kneejoint and articular cartilage

(A). Knee articular cartilage with a cartilage lesion. (B). Magnified view of articular cartilage with specific zones indicated and a magnified view of the contents of the middle layer of articular cartilage. 


\subsection{Cartilage Extracellular Matrix}

The articular cartilage ECM consists of a collagen network which is comprised of primarily Collagen type II (Col2a1), and additionally of collagens type IX (Col9a1) and XI (Col11a1) which help to form and stabilize the collagen type II fibril network ${ }^{8-11}$. Minor quantities of Col6a1, Col12a1, Col14a1 and Col27al are also found in cartilage ${ }^{12}$. The collagen network is surrounded by a highly hydrated aggregation of proteoglycans and other glycoproteins (Figure 1.2A). Glycoproteins and proteoglycans as COMP (cartilage oligomeric protein), Matrilin1 (Matn1/Crtm), perlecan (Hspg2), versican (Vcan), decorin (Dcn), biglycan (Bgn) and fibromodulin (Fmod) are characterized by their ability to interact with and support the collagen fibril network and retention and transport of growth factors $^{13,14}$. Aggrecan (Acan) is the main proteoglycan and forms macromolecular complexes by binding to hyaluronan via link proteins and binding of glycosaminoglycans (GAGs), such as chondroitin sulfate and keratan sulfate (Figure 1.2B). The glycosaminoglycan side chains of the proteoglycans are composed of repeating disaccharide units carrying negatively charged sulphate and carboxyl groups. The resulting fixed negative charge density attracts mobile cations and water into the ECM and thus provides in the elastic properties of the tissue ${ }^{15,16}$. In addition to resisting compressive forces and providing lubrication during movement, the high water retention capacity of hyaline cartilage also supports in distributing nutrients to chondrocytes. The proteoglycan aggregations, together with the quality of the collagen network determine the strength and flexibility of the cartilage tissue and ability to withstand repetitive compressive forces for which articular cartilage has been designed $^{17-20}$.
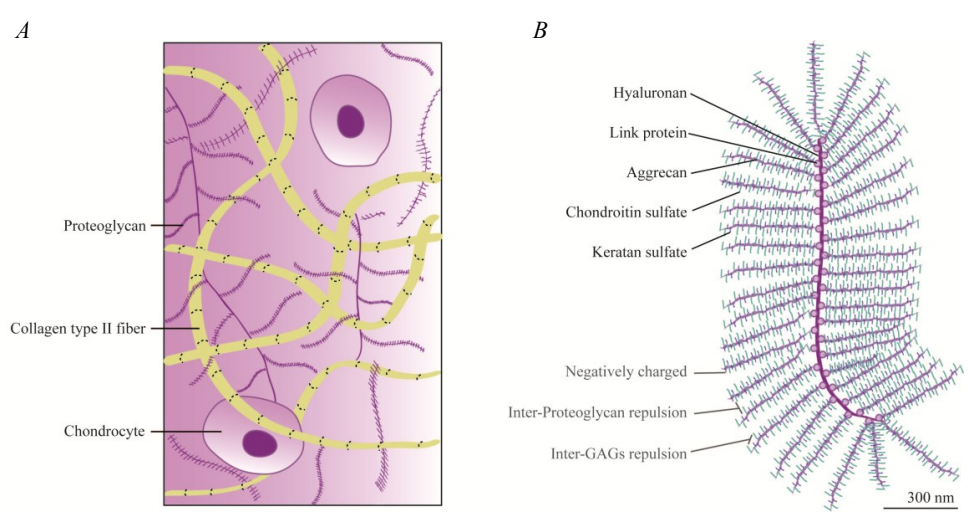

Figure 1.2: Composition of the cartilage extracellular matrix

(A). Representation of different components of the ECM, including the collagen fibril network, proteo-glycans and chondrocytes. $(B)$. Proteoglycans have the appearance of "bottle brush" structure. Typically they contain GAG sidechains of chondroitin and keratan sulfate and are linked to a hyaluronan backbone via link proteins. 


\subsection{Chondrocytes and Macromolecular Structure of the ECM}

Chondrocytes residing in the ECM provide maintenance of the articular cartilage tissue. Each chondrocyte is entrapped in its own excreted matrix and is responsible for the matrixturnover in its own vicinity and its viability depends on an optimal bio-chemical and mechanical environment. Based on collagen fibre orientation, chondrocyte shape and distribution and biochemical composition ${ }^{1,3,4}$, four zones can be distinguished in articular cartilage (Figure 1.3). In the superficial zone, chondrocytes are flattened and are surrounded by a thin layer of ECM, mainly composed of collagen type II -fibres. The fibres are oriented parallel to the articular surface and have a relatively low content of proteoglycans, which results in high tensile stiffness and the ability to distribute load over the surface and protecting the deeper layers. In the transitional middle zone the cells and collagen fibres appear dispersed randomly ${ }^{21,22}$. Here, high concentrations of proteoglycans enable the tissue to bear compressive forces. In the deep zone, chondrocytes are grouped radially in columns and thicker collagen fibres are arranged perpendicular to the articular surface, providing the greatest resistance to compressive forces. In the calcified zone, (hypertrophic) chondrocytes are distributed sparsely and are surrounded by a calcified matrix. The calcified layer plays an integral role in securing the cartilage layer to the subchondral bone by anchoring the collagen fibrils to the subchondral bone tissue. The junction between uncalcified and calcified cartilage is called the "tidemark". At the tidemark shear stresses are converted into compressive forces which are in turn transmitted to the subchondral bone ${ }^{23}$.

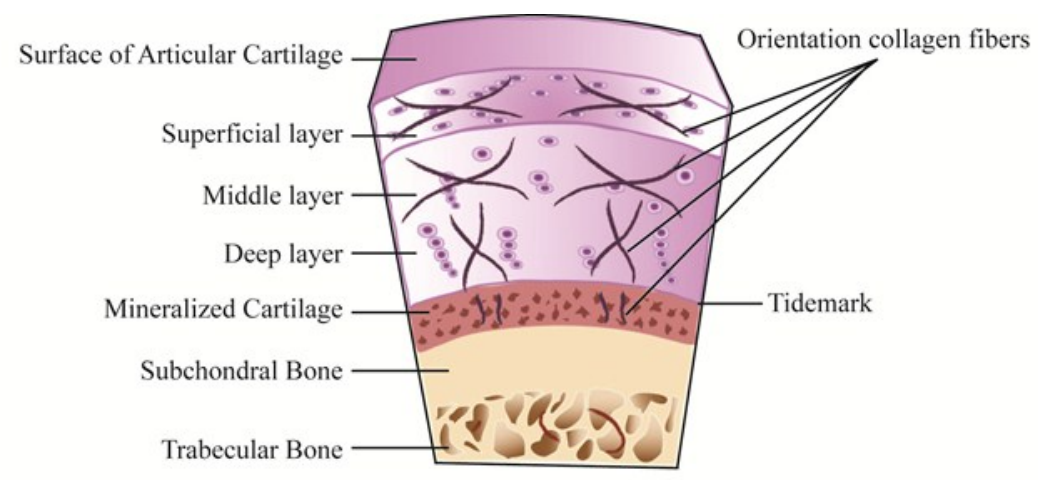

Figure 1.3: Macromolecular structure of the cartilage extracellular matrix

Schematic macroscopic view on the different layers of articular cartilage with the collagen fiber orientation is depicted. 


\section{Cartilage defects and treatment of damaged cartilage}

Cartilage defects can arise due to trauma or cartilage degeneration, but are generally difficult to diagnose ${ }^{24,25}$. Since cartilage has no nerve fibres, cartilage lesions often present with only (minor) effusion of the affected joint or without symptoms at all. Symptoms as joint pain, locking phenomena and reduced or disturbed joint-function may arise from other tissues structures likely to be damaged upon trauma (e.g. subchondral bone, ligaments or menisci). Although the occurrence of chondrocyte progenitor cells has been reported in the superficial layer of articular cartilage ${ }^{26,27}$, cartilage has a limited ability for self-repair ${ }^{28,29}$. This was already recognized in 1743 when the British surgeon William Hunter made the statement: "From Hippocrates to the present age it is universally allowed that ulcerated cartilage is a troublesome thing and that once destroyed it is not repaired" ${ }^{30}$.

Small focal lesions will gradually increase not only in girth but also in length, ultimately leading to deterioration of the entire thickness of the articular cartilage layer. This process is initiated by loss of extracellular matrix eventually followed by loss of the chondrocytes. When unnoticed and/or left untreated, this will lead to destabilization of the joint and ultimately to the onset of osteoarthritis (OA) (Figure 1.4). This consequence is one of the main reasons for clinicians and researchers to find ways for repairing cartilage defects before OA is able to develop.

A

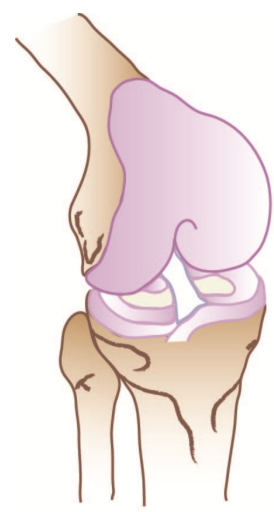

Healthy knee joint
B

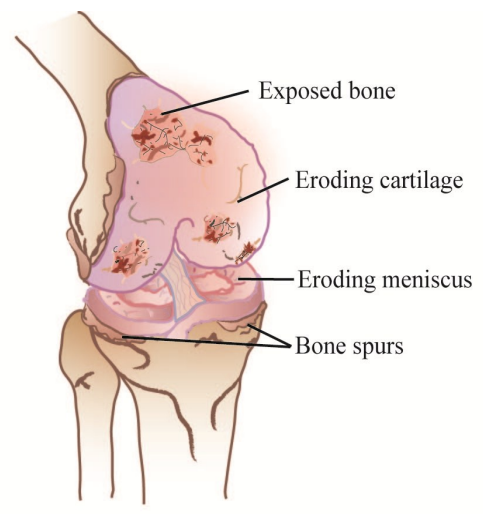

Erosion of cartilage and bone spurring in knee joint

Figure 1.4: Healthy versus Osteoarthritic cartilage

View on healthy articular cartilage of the knee (A) and of a knee with osteoarthritis (B). 
Treatment of damaged cartilage can be grouped to four concepts of principle; the four $\mathrm{R}^{\prime} \mathrm{s}^{31}$. The joint surface can be; (i) replaced, (ii) relieved, (iii) resected or (iv) restored/ repaired. A joint prosthesis is an example of joint replacement, joint distraction and osteotomies can induce joint relieve. Osteotomies are used to re-align the axis of loading in patients with a malalignment of the leg. By transferring the load to the less affected cartilage (e.g. previously less loaded/damaged cartilage) the damaged part is relieved. Arthodesis is an example of joint resection. Cartilage restoration implies methods to heal or regenerate the joint surface, with or without the subchondral bone, into healthy hyaline articular cartilage to restore joint functioning. As such, the definition of cartilage restoration resembles the definition of regenerative medicine (RM): "regenerative medicine replaces or regenerates human cells, tissues or organs, to restore or establish normal function ${ }^{, 32}$. Current cartilage regenerative medicine approaches can be subdivided into clinical approaches and laboratory based approaches (Figure 1.5).

One of the main problems in cartilage regenerative medicine is unwanted mineralization (and formation of intralesional osteophytes) of the cartilage ${ }^{33,34}$. As described above, the properties of the hyaline cartilage matrix are essential to withstand the repetitive compressive forces of movement. Hypertrophic cartilage or mineralized cartilage in the articular surface has inferior properties concerning resisting repetitive mechanical loading and will thereby result in the further deterioration of the joint cartilage and can act as a source of pain $^{35}$. Hypertrophic differentiation of chondrocytes is thus of concern in cartilage repair techniques but also in the onset of osteoarthritis, as markers for hypertrophic differentiation are specifically expressed at early stages of $\mathrm{OA}^{36-38}$.In addition to formation of hypertrophic cartilage, stimulating progenitor cells towards extracellular matrix producing chondrocytes and keeping them in their desired differentiation state is another important factor to consider in cartilage repair techniques ${ }^{33,34}$. 


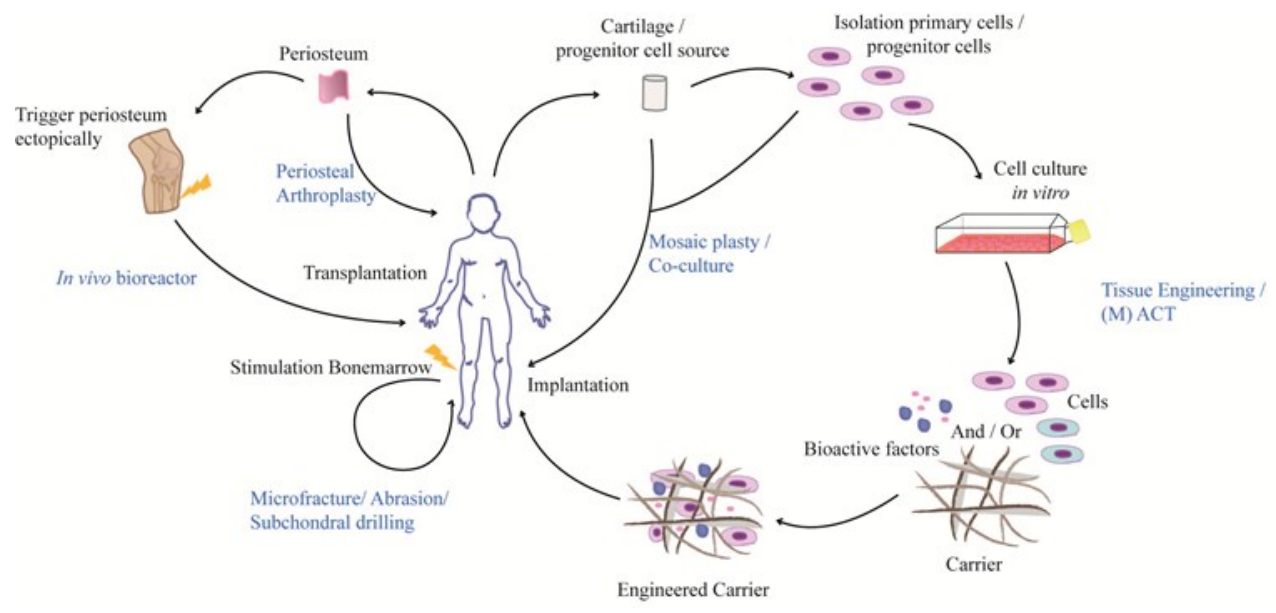

Figure 1.5: Articular cartilage regenerative medicine

Schematic representation of principles of different cartilage regenerative medicine approaches.

\subsection{Clinical Approaches in Cartilage Regenerative Medicine}

Bone marrow stimulating techniques as microfracture, abrasion and subchondral drilling are relatively easy applicable, cheap and reliable methods to approach the functional repair of cartilage defects. These techniques are based on the penetration of the subchondral bone allowing ingress of bone marrow stem cells into the damaged cartilage (Figure 1.6A) ${ }^{39-43}$. These cells are thought to differentiate into the chondrogenic lineage and functional ECMproducing chondrocytes, thereby resulting in repair of the cartilage defect. However, formation of fibrocartilage and calcification of repaired tissue are hampering clinical outcome on the long term ${ }^{34,43}$. Another source of mesenchymal progenitor cells can be found in the cambium layer of the periosteum and in the perichondrium. These cells have been described to have a chondrogenic potential as well ${ }^{44-48}$ Covering cartilage defects with periosteum derived grafts (periosteal arthroplasty) is therefore another explored strategy to treat cartilage defects (Figure 1.6B) (9-54 $^{4 n}$ short term, results were found to be quite promising with regard to cartilage repair ${ }^{50-54}$. Unfortunately, on the long term results were poor and failure was related to calcification of the grafts ${ }^{49}$.

Other techniques imply the transplantation of mature chondrocytes or cartilage such as mosaicplasty (Osteochondral Autograft Transfer System; OATS), allografts and Autologous Chondrocyte Transplantation (ACT). Mosaicplasty or OATS involves harvesting one or more osteochondral plugs from a relatively less weight-bearing region of the joint and subsequent implantation of these plugs into the articular cartilage defect (Figure 1.6C) ${ }^{55-58}$. 


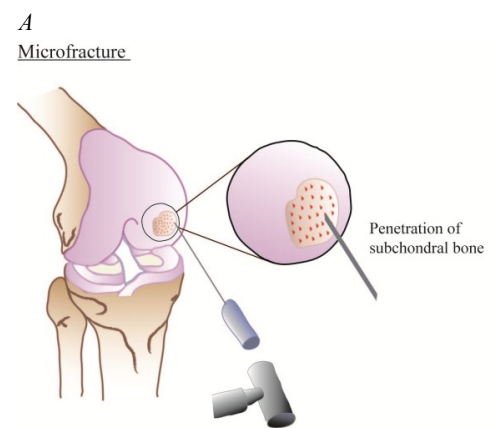

B Periosteal arthroplasty

Mosaic plasty

Autologous Chondrocyte Transplantation
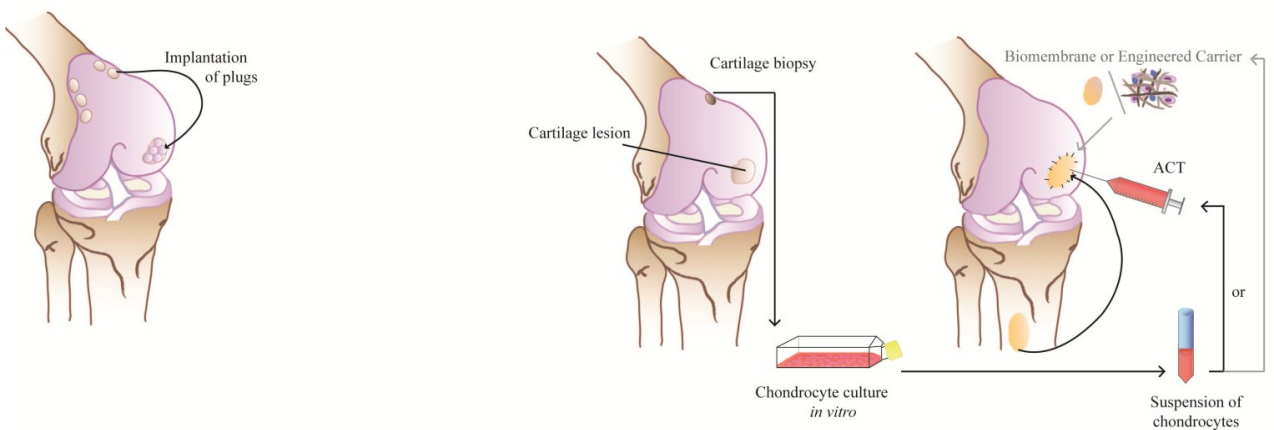

Figure 1.6: Current clinical cartilage repair approaches

(A). Schematic representation of the microfracture procedure. (B). Schematic representation of the periosteal arthroplasty procedure. $(C)$. Schematic representation of the mosaic plasty procedure. $(D)$. Schematic representation of procedure of autologous chondrocyte transplantation (ACT) or chondrocyte transplantation with an engineered carrier.

The use of allografts can overcome possible donor site morbidity ${ }^{57,59-61}$ or shortage of graft material. ACT refers to a cell based cartilage repair procedure where cartilage is harvested arthroscopically from a less weight-bearing region of the joint and transferred to a specialized laboratory where the chondrocytes are enzymatically released from their matrix and expanded in vitro. The patient then undergoes a second operation where the in vitro expanded chondrocytes are re-implanted at the damaged site of the articular cartilage, in combination with a membrane (periosteum or collagen-based membrane) (2-64 $^{\text {or pre-seeded }}$ in a matrix (Matrix Assisted Chondrocyte Transplantation; MACT) (Figure 1.6D) ${ }^{65}$. Nevertheless, the use of these techniques is restricted due to a limited availability of autologous cartilage (mosaicplasty) or donors, possible disease transfer (allografts), or expensive and time consuming logistics and culture methods (ACT). Furthermore, cartilage hyper- 
trophy is also seen after ACT, albeit more in the periosteum covered ACT than in matrixassisted $\mathrm{ACT}^{66,67}$.

\subsection{Cartilage Tissue Engineering / Laboratory-based Regenerative Medicine}

The science of combining cells with carrier materials to reproduce tissues is called Tissue Engineering (TE) (Figure 1.5). Carrier material or scaffold refers to a wide variety of artificial two-dimensional (2D) or three-dimensional (3D) structures that are designed for the purpose of tissue engineering. Carrier materials may be seeded with cells before implantation or are designed to recruit or retain cells at the desired place. Different variables are important parameters for carrier design: pore diameter, shape, kind of material, (bio) degradability, implantation site, functionalization, mechanical stability and others. When applying carrier materials for cartilage regenerative purposes, some authors find enhanced cartilage healing, others conclude that scaffolds have a limited usefulness for chondrocyte grafting in large defects ${ }^{68-71}$. New generation experimental scaffolds are loaded with bioactive factors (e.g. growth and differentiation factors) that directly influence the local implantation environment ${ }^{72-74}$. Although it has already led to fruitful achievements, TE created cartilaginous tissues are not generated on a large scale ${ }^{63,75,76}$ and an ideal material for carrier material for cartilage regeneration has not been identified yet, as the biological processes involved are far more complex than anticipated. In addition, time consuming and expensive culture procedures and logistics, multiple operations and quality of the tissue repair initiated by tissue engineered constructs (e.g.: unwanted calcification and formation of fibrocartilage) remain important drawbacks.

Recently a shift can be observed in the attempts to repair tissues in general. This shift includes more specific natural stimuli which trigger and enhance the regenerative capacity of the tissue itself. Examples are injection/stimulation of stem cells or progenitor cells (cell therapies) and the induction of local regeneration by biologically active molecules. Our group was recently successful in such an approach by injecting an agarose gel into the space between bone and periosteum, which initiated chondrogenic differentiation of the locally residing mesenchymal progenitor cells and resulted in cartilaginous tissue formation. The grown tissue was harvested during the chondrogenic phase and successfully used to repair osteochondral defects (Figure 1.7) ${ }^{77}$. 
$A$

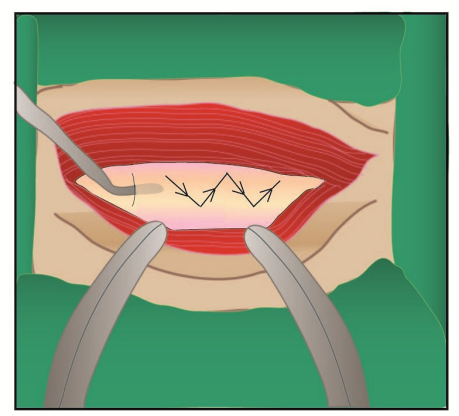

Sub-periosteal administration of biogel
$B$

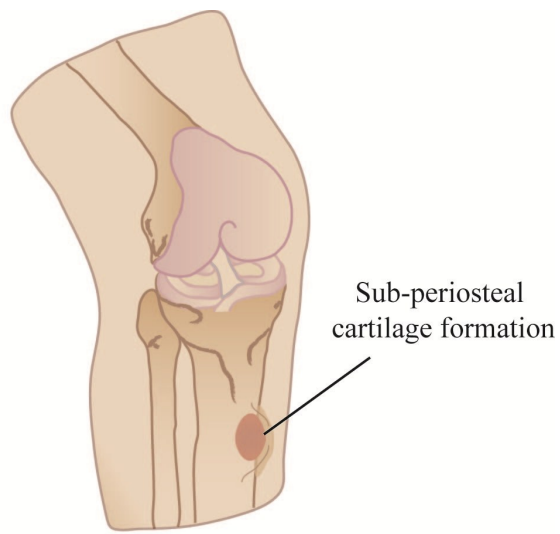

Formation of callus (2 weeks)

C
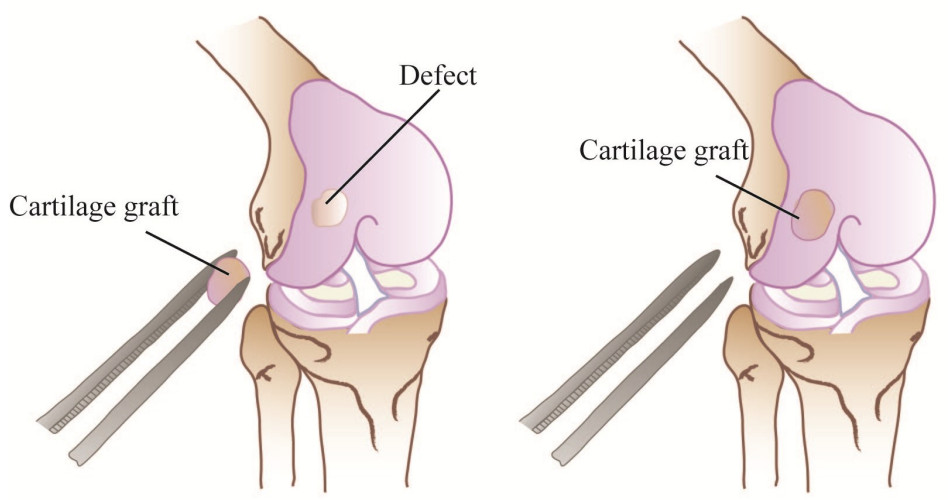

Implantation of cartilage graft in defect

\section{Figure 1.7: The in vivo bioreactor}

The in vivo bioreactor (IVB) is a strategy for de novo engineering of ectopical autologous cartilage within the subperiosteal space. (A). An agarose gel is injected in the subperiosteal space of the upper medial part of the rabbit tibia. (B). After two weeks callus formation becomes visible at the tibia. $(C)$. The IVB-cartilage graft can be press-fit implanted in an osteochondral defect of the medial femur condyle of the same rabbit ${ }^{28,77,78}$ 


\section{Chondrogenic differentiation}

Chondrogenic differentiation encompasses the commitment and differentiation of chondroprogenitor cells towards chondrocytes (see Figure 1.8). In vivo, chondrogenic differentiation is almost exclusively initiated from local mesenchymal progenitor cells that reside in cartilaginous tissue (growth plate resting zone or the articular cartilage superficial layer $^{79,80}$ ) or in surrounding fibrous tissues (e.g. periosteum ${ }^{47,81}$ ). Ex vivo however, chrondrogenic differentiation has been reported from various primary (mesenchymal) progenitor cell sources including synovial fluid/membrane, adipose tissue, induced pluripotent stem cells (iPS ${ }^{82}$ ), bone marrow and many more ${ }^{83}$.

In addition to providing articulating joint surfaces with functional cartilage and maintaining cartilage integrity, chondrogenic differentiation also plays an essential role during endochondral ossification. Endochondral ossification underlies skeletogenesis and bone fracture healing and is a developmental process during which cartilaginous primordia are gradually replaced by bone tissue. Growth plate chondrocytes originating from the resting zone or fracture callus chondrocytes originating from mesenchymal progenitors gradually proliferate and produce a cartilaginous matrix. When exiting the cell cycle they differentiate into mineralized hypertrophic chondrocytes which finally die by apoptosis. The remaining mineralized extracellular matrix provides a molecular scaffold for infiltrating osteoblasts and osteoclasts to adhere to and remodel, setting the stage for de novo bone deposition ${ }^{84,85}$. Notably, chondrocytes in articular cartilage retain their chondrocyte phenotype and, except for chondrocytes near the tidemark, normally do not further differentiate into hypertrophic chondrocytes, probably due to the local microenvironment. Unfortunately, as a natural result of this endochondral ossification process, in vitro chondrogenic differentiation of progenitor cells for cartilage regenerative purposes tends to progress into hypertrophic differentiating chondrocytes. 


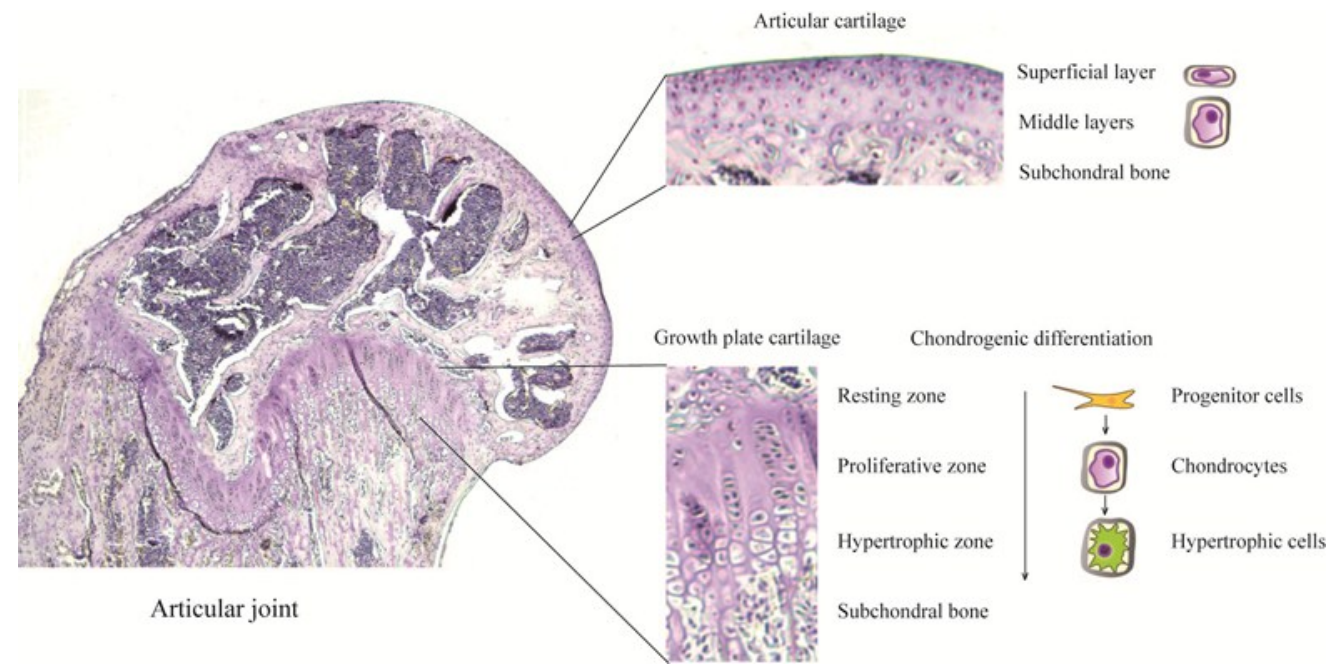

Figure 1.8: Chondrogenic differentiation

Hematoxylin stained section of the growth plate near the articular joint from the proximal tibia of a six week old mouse. Different phases of chondrogenic differentiation are visible and the differnt layers of growth plate and articular cartilage are indicated.

\subsection{Transcriptional Regulators in Chondrogenic Differentiation}

Chondrogenic differentiation starts when mesenchymal progenitor cells are triggered to differentiate into the chondrogenic lineage (Figure 1.9). Chondrogenic progenitor cells express typical ECM and cell adhesion molecules like tenascin c (Tnc), syndecan 3 (Sdc3), $\mathrm{N}$-cadherin (Ncad) and Ncam1 (neural cell adhesion molecule 1). One of the first key important chondrogenic differentiation regulatory events is activation of the Sox-trio transcription factors; Sox9 (SRY-(sex determining region Y)-box9) in combination with L-Sox 5 and Sox 6 are responsible for commitment and differentiation in the chondrogenic lineage ${ }^{14,86,87}$. Sox 9 was originally identified as the causative gene in Campomelic dysplasia, a severe skeletal dysplasia associated with XY sex reversal and disproportionally short stature as well as general lack of cartilaginous tissue formation ${ }^{88,89}$. Sox9 was found to be essential for murine early chondrogenic lineage determination as well ${ }^{90}$. Upon nuclear occupation, Sox 9 binds as a homodimer to its consensus recognition sequence $(A / T)(A / T)$ CAA(A/T)G, which includes the highly conserved AACAAT motif recognized by the high mobility group (HMG)-box domain shared amongst SOX and SRY proteins ${ }^{86,91-93}$. During chondrogenic differentiation Sox 9 drives the transcription of and cooperates with L-Sox 5 and Sox6 for efficient transcription of the important ECM genes Col2al and Acan ${ }^{87,90,94-96}$. 
Other ECM genes have also been shown to be under transcriptional control of Sox9, of which Col9a1, Col27a1 and Matn1 are important ones ${ }^{97-100}$. Another important transcriptional regulator of early chondrogenic differentiation is Bapx1/Nkx3.2 (bagpipe homeobox homolog 1/NK3 homeobox 2). Interestingly, Bapx1/Nkx3.2 and Sox9 are able to induce each other's expression which results in sustained chondrogenic differentiation ${ }^{101}$. Bapx $1 /$ Nkx3.2 functions as a transcriptional repressor of the main hypertrophic transcription factor Runx2 (runt-related transcription factor 2) and is expressed during chondrogenic differentiation but lost upon chondrocyte hypertrophy ${ }^{102-104}$. Sox 9 and Bapx1/Nkx3.2 are thus negative regulators of chondrocyte hypertrophy. The proliferative capacity of the differentiating chondrocytes is maintained by transcription factors, such as members of the activating transcription factor (Atf) and cyclic AMP response element binding protein (CREB) family ${ }^{105,106}$. Subsequently, the proliferative chondrocytes in growth plates exit the cell cycle and subsequently undergo a remodeling of their extracellular matrix and increase in cell volume up to ten times ${ }^{107}$ further differentiating into hypertrophic chondrocytes, which is in contrast to articular chondrocytes which will normally not progress into the hypertrophic phase.

Hypertrophic differentiation is accompanied by increased expression of Runx 2 and Mef2c (Myocyte-specific enhancer factor 2C), which are important transcription factors for collagen type X (Col10a1), the main collagen found in hypertrophic chondrocytes ${ }^{108-111}$. Mice lacking Runx 2 exhibit a disturbance of chondrocyte maturation as demonstrated by an absence of Col10al expression ${ }^{112-114}$. These abnormalities could be reversed by transgenedriven expression of Runx 2 in chondrocytes. Transgenic expression of Runx 2 in wild-type mice not only accelerates hypertrophy in normal chondrocytes but even induced hypertrophy in cartilage that normally never undergoes hypertrophy, such as cartilage of tracheal rings ${ }^{115,116}$, further stressing on the key role of Runx2 in regulating chondrocyte hypertrophic development.

In addition, hypertrophic chondrocytes also express vascular endothelial growth factor A (VEGF-A) to stimulate vascular ingrowth. The transcription of VEGF-A is regulated by Runx2 and Mef2 $\mathrm{c}^{14,117}$. Also several MMPs (matrix metalloproteinases) and ADAMTSs (a disintegrin and metalloproteinase with thrombospondin motifs) for breakdown of the ECM are synthesized ${ }^{14,118}$. At the final stage of hypertrophic differentiation several mineralization proteins are expressed, such as Alp (alkaline phosphatase) and osteopontin (Opn, also known as bone sialoprotein I (Bsp1)), which mineralize the extracellular matrix ${ }^{14,119}$. Finally, the hypertrophic chondrocytes die by apoptosis, leaving their mineralized extracellular 
matrix behind for osteoblasts to adhere, which will eventually remodel the matrix into bone tissue.

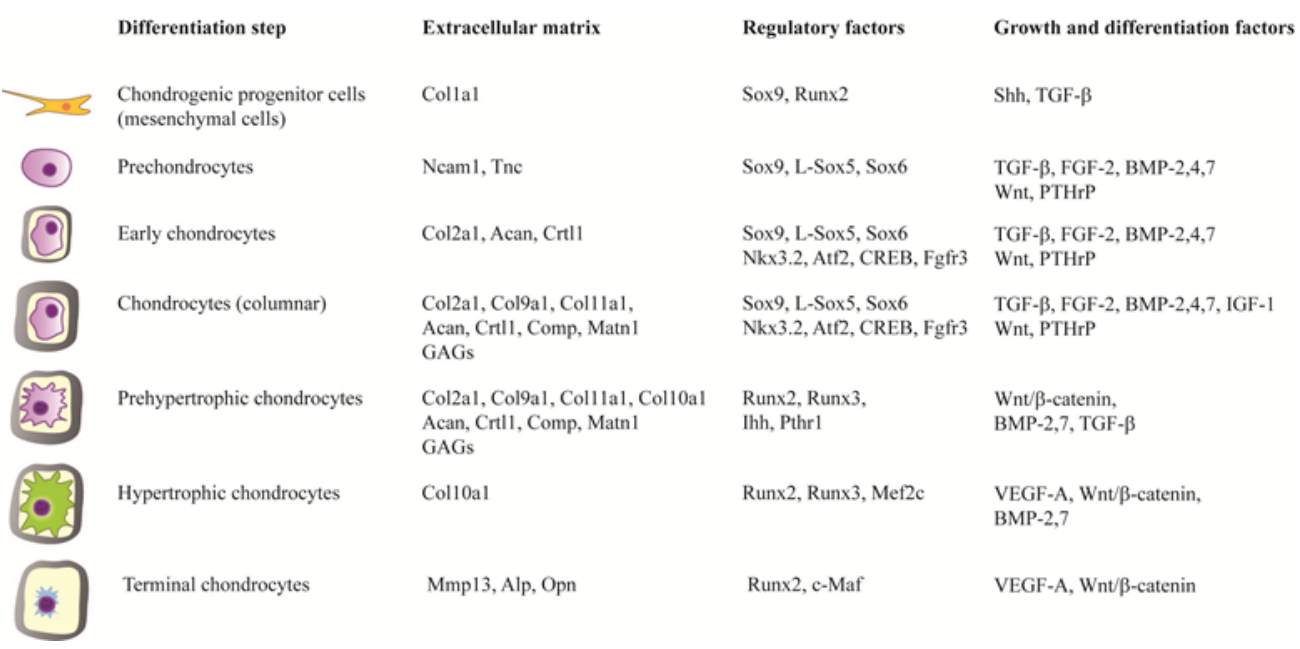

Figure 1.9: Chondrogenic differentiation

Successive steps of chondrogenic differentiation during endochondral ossification with schematic representation of the cells, major extracellular matrix genes, regulatory factors and growth and differentiation factors indicated at each step. (Adapted from: $:^{14}$ )

\subsection{Growth Factors and Paracrine Regulators in Chondrogenic Differentiation}

In growth plate development as well as in the development and homeostasis of articular cartilage several signaling pathways are interacting or shared between the different tissues. Indian hedgehog (Ihh) and parathyroid hormone related peptide (PTHrP) coordinate chondrocyte proliferation and differentiation in the paracrine PTHrP-Ihh feedback loop ${ }^{85}$. PTHrP is synthesized by proliferating chondrocytes and perichondrial cells ${ }^{85}$ and maintains chondrocyte proliferation by activating Cyclin D $1{ }^{120}$ and prevents premature hypertrophy by inducing Cyclin D1-mediated degradation of Runx ${ }^{121}$. Proliferating chondrocytes located at a sufficient distance from the PTHrP source stop proliferating and become hypertrophic, Ihh synthesizing cells ${ }^{122}$. Ihh is expressed by prehypertrophic chondrocytes and accelerates the (hypertrophic) differentiation of proliferative chondrocytes and additionally it increases the expression of PTHrP, resulting in a feedback loop that controls the pace of chondrocyte proliferation and maturation ${ }^{123-125}$. Next to the PTHrP-Ihh loop, fibroblast growth factors (FGFs) crucially regulate chondrocyte proliferation and differentiation possibly by stimulating Sox 9 expression and inhibiting proliferation and Ihh expression ${ }^{85}$. 
FGF signaling is balanced by bone morphogenic protein (BMP)- signaling ${ }^{126}$. BMPs are described to have multiple roles during bone and cartilage formation, as well as growth plate development ${ }^{127}$. Interestingly; BMPs were initially discovered because of their remarkable ability to ectopically induce endochondral bone formation ${ }^{128}$. In a cartilage context, BMPs are involved in stimulating early chondrogenesis, cartilage maintenance and hypertrophic differentiation ${ }^{127}$. Especially BMP-2, BMP-4 and BMP-7 (OP-1) have been demonstrated to promote chondrogenic differentiation in vitro ${ }^{127}$. BMPs belong to the transforming growth factor beta (TGF- $\beta$ ) superfamily, which are important regulators of differentiation, proliferation, tissue homeostasis and -repair in general. TGF- $\beta$ isoforms (TGF- $\beta 1$, TGF- $\beta 2$ and TGF- $\beta 3$ ) support the differentiation of mesenchymal progenitor cells into the chondrogenic lineage ${ }^{129-134}$. The TGF- $\beta$ isoforms mainly signal through phosporylated R-Smads, which in combination with co-(transcriptional) factors regulate specific target-gene expression ${ }^{135,136}$. Related to its chondrogenic properties, TGF- $\beta$ signalling is also involved in the formation of osteophytes during $\mathrm{OA}^{137-140}$. Another important regulator of chondrogenic differentiation is the canonical Wnt (wingless-type MMTV integration site family)/ $\beta$-catenin signalling pathway. Upon binding of a Wnt ligand to its receptor (Frizzled), cytosolic $\beta$-catenin translocates to the nucleus where it forms complexes with transcription factors such as the TCF/LEF (transcription factor/ lymphoid enhancer-binding factor) family and thereby regulates downstream target-gene expression. In absence of the Wnt signal cytosolic $\beta$-catenin is phosphorylated by GSK-3 $\beta$ (glycogen synthase kinase $3 \beta$ ) and subsequently degraded ${ }^{141-143}$. Members of the canonical $\mathrm{Wnt} / \beta$-catenin signalling pathway are generally expressed during hypertrophy and accordingly also promote chondrocyte hypertrophy, presumably via the TCF/LEF binding site in the promoter region of the Runx 2 gene ${ }^{144-146}$. In early chondrogenic differentiation Sox9 interacts with $\beta$-catenin and promotes its phosphorylation and thereby degradation thereby preventing osteoblastic or hypertrophic differentiation ${ }^{144,147-150}$.

\section{Tissue Repair Processes and Chondrogenic Differentiation}

Joint homeostasis is essential during cartilage repair, but methods for improving joint homeostasis in cartilage repair techniques are hardly addressed ${ }^{151,152}$. Addressing the condition of the cartilage microenvironment may not only be the key to a new generation of 
bone marrow-based techniques to regenerate hyaline cartilage ${ }^{153}$, but may also improve cartilage repair in general. While inflammatory processes are generally seen as a negative factor in cartilage and joint homeostasis and are contributing factors in OA and rheumatoid arthritis (RA), inflammation is also known to be the first and essential phase of tissue repair in general. This suggests that inflammatory processes could be relevant pathways for addressing cartilage tissue repair. Supporting data for this notion is found in bone fracture healing processes where haematoma formation and injury-induced inflammatory responses are essential for fracture healing and its accompanying chondrogenic differentiation / endochondral ossification. (Figure 1.10) ${ }^{154-156}$. This essential inflammatory response induces local expression of extracellular signalling molecules like TGF- $\beta 1$, BMPs, insulin-like growth factor (IGF)-1 and platelet derived growth factor (PDGF), which regulate chondrogenic differentiation processes ${ }^{157,158}$. In contrast to their wellknown catabolic effects in pathological conditions such as OA and RA, several inflammatory cytokines and chemokines (e.g. interleukin-1 (Il-1), Il-6, tumor necrosis factor alpha $(\mathrm{TNF} \alpha)$, prostaglandin $\mathrm{E}_{2}\left(\mathrm{PGE}_{2}\right)$ and nitric oxide $\left.(\mathrm{NO})\right)$ are essential for bone fracture repair ${ }^{155,158-160}$. TNF $\alpha$ signalling-deficient mice show impaired fracture healing due to delayed early chondrogenesis and chondrocyte apoptosis ${ }^{161,162}$. Il-6 knock-out (KO) mice show delayed cartilage callus maturation and impaired mineralization after inducing bone fracture ${ }^{163,164}$. Expression of the $\mathrm{PGE}_{2}$ synthesizing enzyme cyclooxygenase-2 (COX-2) has been observed during fracture callus formation as well as in the developing growth plate, pointing towards a role in endochondral ossification ${ }^{165-167}$. COX-2 knock-out mice exhibit impaired fracture healing due to lack of $\mathrm{PGE}_{2}$ synthesis ${ }^{168}$. Similarly, inducible nitric oxide synthase (iNOS), responsible for NO synthesis during acute inflammation, is thought to have a pivotal role in bone fracture repair as well ${ }^{169}$. Expression of iNOS is observed in fracture callus and iNOS -/- mice display impaired fracture healing ${ }^{170-172}$. One of the key players in the transcriptional regulation of these inflammatory genes is nuclear factor kappa-light-chain-enhancer of activated B-cells (NF- $\kappa \mathrm{B})$. NF- $\kappa \mathrm{B}$ signalling can be activated via several receptors-pathways, amongst which classically the activation of Toll-like receptor (TLR)-2/-4 and the TNF $\alpha$ receptor ${ }^{173-176}$. In addition, also the TGF- $\beta$ receptor and IGF receptor are described to induce NF- $\kappa B$ signalling ${ }^{177,178}$. Upon receptor activation, the cytoplasmically localized NF- $\mathrm{KB}$ complex translocates to the nucleus, leading to NF- $\kappa \mathrm{B}$-mediated transcription of inflammatory target genes ${ }^{174,175}$. Besides its functions in transcriptional regulation of catabolic inflammatory processes, NF- $\mathrm{BB}$ has been

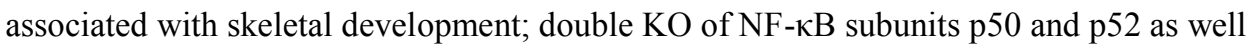


as p65 -/- mice show retarded skeletal development ${ }^{179,180}$. Previous studies have established an important role for inflammatory mediators during endochondral bone formation, however mainly focused on the osteogenic phase or complete process of endochondral ossification. Thusfar a potential involvement of inflammatory signalling in chondrogenic differentiation remains poorly studied. The NF- $\kappa B$ subunit $\mathrm{p} 65$ was recently identified as a transcription factor for Sox9, the major chondrogenic transcription factor ${ }^{181}$. In addition to Sox9, expression of BMP-2 in chondrocytes is co-regulated by NF- $\mathrm{KB}^{182}$. Also, BMP-2 expression is under control of pro-inflammatory cytokines TNF $\alpha$ and Il- $1 \beta^{183,184}$.

Taken together there is a growing body of experimental evidence, showing that inflammatory mediators and their down-stream pathways are not only associated with cartilage degeneration, but are also crucially involved in the chondrogenic differentiation process during endochondral ossification in vivo. These insights could be explored for cartilage repair technologies to increase the differentiation potential of progenitor cells toward cartilaginous tissue and establishing optimal joint homeostasis after cartilage repair to increase the success of the treatment.

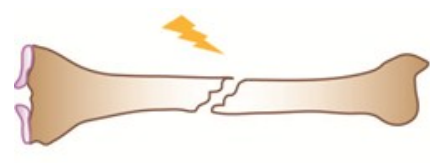

Impact

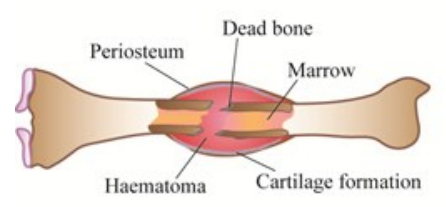

Inflammation

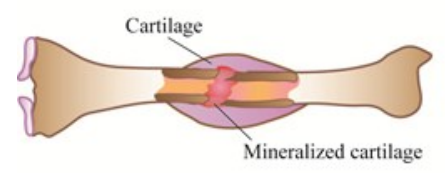

Soft callus

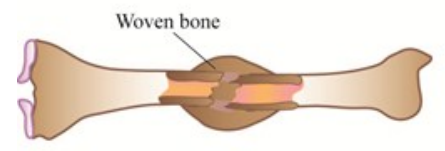

Ossification

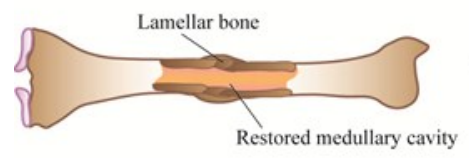

Remodelling
Figure 1.10: Phases of endochondral fracture repair Schematic representation of different phases of bone fracture healing. First an inflammatory haematoma is formed and mesenchymal cell proliferation begins throughout the affected bone almost directly after injury. Secondly a cartilaginous callus is formed from this tissue which differentiates further along the endochondral ossification pathway to woven bone. The woven bone is gradually replaced by lamellar bone and the fracture is united. The final phase is remodeling, where the shape of the bone is gradually returned to the orginal bone. The latter process may take several years. 


\section{Aims and outline of this thesis}

Chondrogenic differentiation is a tightly regulated process in which many factors regulate the discrete stages of the differentiation program. Understanding the mechanisms of cell commitment and further differentiation into the chondrogenic lineage is of great importance to optimize (progenitor) cell-based cartilage repair techniques. The work described in this thesis aimed to investigate how chondrogenic differentiation capacity from progenitor cells can be influenced via pathways that were unprecedented to be involved in determining the fate of the chondrogenic differentiation program.

Chapter 2 focuses on the involvement of key inflammatory complex NF- $\mathrm{kB} / \mathrm{p} 65$ in chondrogenic differentiation of progenitor cells. We questioned if $\mathrm{NF}-\kappa \mathrm{B} / \mathrm{p} 65$ signalling is involved in early chondrogenic differentiation and if modulating the activity of NF- $\mathrm{kB} / \mathrm{p} 65$ alters the chondrogenic outcome via signalling through key chondrogenic transcription factor Sox9.

$\mathrm{NF}-\mathrm{\kappa B}$ is a member of the Rel family of transcription factors, which also consists of Nuclear factor of activated T-cells 5 (Nfat5). Nfat5 was recently shown to be essential in the tonicity-responsiveness of human articular chondrocytes (HACs). HACs increase their matrix synthesis under increased tonicity of the culture medium via an Nfat5-dependent mechanism ${ }^{185}$. However, the effects of increased tonicity and the involvement of Nfat5 in chondrogenic differentiation of progenitor cells are largely unknown. We hypothesized in Chapter 3 that Nfat5 is involved in chondrogenic differentiation of progenitor cells in vitro and that the differentiation process can be enhanced by increasing the tonicity of the culture medium.

Expression of several growth factors, including BMP-2, is co-regulated in differentiating chondrocytes by NF- $\mathrm{KB} / \mathrm{p} 65$ and in turn BMP-2 is able to regulate $\mathrm{NF}-\kappa \mathrm{B} / \mathrm{p} 65$ signalling which could, at least in part, explain the pro-chondrogenic effect of BMP- $2^{182,186}$. The recombinant version of BMP-2 is clinically approved and used to promote osteogenesis at sites of poor fracture healing (non-unions) and as additional factors to enhance integration of bone grafts. Also, BMP-7 (OP-1) is clinically approved to promote bone healing. A comparison of the in vitro chondrogenic characteristics of BMP-2 versus BMP-7 on differentiation of progenitor cells has not been reported and would warrant evidence-based use of one or the other BMP for specific chondrogenic purposes (Chapter 4). Cyclooxygenase-2 (COX-2) is a transcriptional target of the NF- $\mathrm{kB} / \mathrm{p} 65$ complex and is involved in inflammatory processes. In line with a role for COX-2 in endochondral ossifi- 
cation, mice lacking COX-2 show impaired fracture healing. However, the function of COX-2 in chondrogenic differentiation from progenitor cells in the context of endochondral ossification remains unclear at present. In Chapter 5 we tested if inhibition of COX-2 activity by non steroidal anti-inflammatory drugs (NSAIDs) is able to decrease the level of chondrocyte hypertrophic differentiation in different endochondral differentiation models. Also, the effect of systemic inhibition of COX-2 activity in vivo on chondrocyte hypertrophic differentiation in rabbit growth plates was determined. Next to COX-2-specific NSAIDs, other NSAIDs specifically inhibit COX-1 or both COX-enzymes. To determine how COX-1-specific NSAIDs act in chondrogenic differentiation as compared to COX-2specific NSAIDs, we compared the effects of specific COX-1 and COX-2 inhibitors in chondrocyte ECM formation and studied if specific prostaglandins (PG) are involved in chondrogenic differentiation (Chapter 6).

Equally important for obtaining an optimal chondrogenic phenotype is not only to stimulate progenitor cells towards chondrocytes but also prevent them from hypertrophy. Chondrocyte hypertrophy is not only an unwanted phenomenon in chondrogenic differentiation from progenitor cells but is also a hallmark of early OA in mature chondrocytes and hampers the outcome of chondrocyte-based cartilage repair approaches. A cell culture system displaying a chondrocyte hypertrophic phenotype can be of importance when studying this process on a molecular basis. To hypertrophic marker expression and establish an human chondrocyte hypertrophic model we compared different redifferentiation models for human articular chondrocytes (HACs) (Chapter 7). Potential chondrogenic -stimulating and hypertrophic-suppressing factors identified in our previous studies can be further verified in such a model.

In Chapter 8 the data described in this thesis are discussed with regard to fundamentally understanding chondrogenic differentiation and their relevance for optimizing progenitor cell-based cartilage regenerative medicine approaches. 
6. References

1 Buckwalter, J. \& Mankin, H. Articular cartilage: tissue design and chondrocyte matrix interactions. AAOS Instr Cours Lect 47, 487-504 (1998).

2 Hasler, E. M., Herzog, W., Wu, J. Z., Muller, W. \& Wyss, U. Articular cartilage biomechanics: theoretical models, material properties, and biosynthetic response. Crit Rev Biomed Eng 27, 415-488 (1999).

3 Mankin, H., Mow, V. \& Buckwalter, J. Articular cartilage structure, composition, and function. (AAOS, 2000).

4 Poole, A. R. et al. Composition and structure of articular cartilage: a template for tissue repair. Clin Orthop Relat Res, S26-33 (2001).

Moreira-Teixeira, L. S., Georgi, N., Leijten, J., Wu, L. \& Karperien, M. Cartilage tissue engineering. Endocr Dev 21, 102-115, doi:10.1159/000328140 (2011). Aydelotte, M. B., Greenhill, R. R. \& Kuettner, K. E. Differences between subpopulations of cultured bovine articular chondrocytes. II. Proteoglycan metabolism. Connect Tissue Res 18, 223-234 (1988).

Schenk, R., Eggli, P. \& Hunziker, E. Articular cartilage morphology. (Raven Press, 1986).

8 Fassler, R. et al. Mice lacking alpha 1 (IX) collagen develop noninflammatory degenerative joint disease. Proceedings of the National Academy of Sciences of the United States of America 91, 5070-5074 (1994).

9 Li, Y. et al. A fibrillar collagen gene, Col11a1, is essential for skeletal morphogenesis. Cell 80, 423-430 (1995).

10 Nakata, K. et al. Osteoarthritis associated with mild chondrodysplasia in transgenic mice expressing alpha 1(IX) collagen chains with a central deletion. Proceedings of the National Academy of Sciences of the United States of America 90, 2870-2874 (1993).

11 Vikkula, M. et al. Autosomal dominant and recessive osteochondrodysplasias associated with the COL11A2 locus. Cell 80, 431-437 (1995).

12 Eyre, D. Collagen of articular cartilage. Arthritis Res 4, 30-35 (2002).

13 Knudson, C. B. \& Knudson, W. Cartilage proteoglycans. Semin Cell Dev Biol 12, 69-78, doi:10.1006/scdb.2000.0243 (2001).

14 Lefebvre, V. \& Smits, P. Transcriptional control of chondrocyte fate and differentiation. Birth Defects Res C Embryo Today 75, 200-212, doi:10.1002/bdrc.20048 (2005).

15 Lesperance, L. M., Gray, M. L. \& Burstein, D. Determination of fixed charge density in cartilage using nuclear magnetic resonance. J Orthop Res 10, 1-13, doi:10.1002/jor.1100100102 (1992).

16 Venn, M. \& Maroudas, A. Chemical composition and swelling of normal and osteoarthrotic femoral head cartilage. I. Chemical composition. Annals of the rheumatic diseases 36, 121-129 (1977).

17 Buckwalter, J. A. \& Mankin, H. J. Articular cartilage: tissue design and chondrocyte-matrix interactions. Instr Course Lect 47, 477-486 (1998).

18 Hasler, E. M., Herzog, W., Wu, J. Z., Muller, W. \& Wyss, U. Articular cartilage biomechanics: theoretical models, material properties, and biosynthetic response. Crit Rev Biomed Eng 27, 415-488 (1999).

19 Lin, Z., Willers, C., Xu, J. \& Zheng, M. H. The chondrocyte: biology and clinical application. Tissue Eng 12, 1971-1984, doi:10.1089/ten.2006.12.1971 (2006). 
20 Poole, A. R. et al. Composition and structure of articular cartilage: a template for tissue repair. Clin Orthop Relat Res, S26-33 (2001).

21 Hunziker, E. Articular cartilage structure in humans and experimental animals. (Raven Press, 1992).

22 Aydelotte, M. \& Kuettner, K. Heterogeneity of articular chondrocytes and cartilage matrix. (Marcel Dekker, 1992).

23 Radin, E. L. et al. Effects of mechanical loading on the tissues of the rabbit knee. J Orthop Res 2, 221-234 (1984).

24 Curl, W. W. et al. Cartilage injuries: a review of 31,516 knee arthroscopies. Arthroscopy 13, 456-460 (1997).

25 Hjelle, K., Solheim, E., Strand, T., Muri, R. \& Brittberg, M. Articular cartilage defects in 1,000 knee arthroscopies. Arthroscopy 18, 730-734 (2002).

26 Dowthwaite, G. P. et al. The surface of articular cartilage contains a progenitor cell population. J Cell Sci 117, 889-897 (2004).

27 Park, Y., Sugimoto, M., Watrin, A., Chiquet, M. \& Hunziker, E. B. BMP-2 induces the expression of chondrocyte-specific genes in bovine synovium-derived progenitor cells cultured in three-dimensional alginate hydrogel. Osteoarthritis Cartilage 13, 527-536 (2005).

28 Emans, P. J., Surtel, D. A., Frings, E. J., Bulstra, S. K. \& Kuijer, R. In vivo generation of cartilage from periosteum. Tissue Eng 11, 369-377 (2005).

29 Mankin, H., Mow, V. \& Buckwalter, J. Articular cartilage repair and osteoarthritis. (American Academy of Orthopaedic Surgeons, 2000).

30 Hunter, W. Of the structure and disease of articulating cartilages. 1743. Clin Orthop Relat Res, 3-6 (1995).

31 O'Driscoll, S. W. The healing and regeneration of articular cartilage. J Bone Joint Surg Am 80, 1795-1812 (1998).

32 Mason, C. \& Dunnill, P. A brief definition of regenerative medicine. Regen Med 3, 1-5, doi:10.2217/17460751.3.1.1 (2008).

33 Dickhut, A. et al. Calcification or dedifferentiation: requirement to lock mesenchymal stem cells in a desired differentiation stage. J Cell Physiol 219, 219-226 (2009).

34 van Osch, G. J. et al. Cartilage repair: past and future--lessons for regenerative medicine. Journal of cellular and molecular medicine 13, 792-810 (2009).

35 van der Kraan, P. M. \& van den Berg, W. B. Chondrocyte hypertrophy and osteoarthritis: role in initiation and progression of cartilage degeneration? Osteoarthritis and cartilage / OARS, Osteoarthritis Research Society 20, 223-232, doi:10.1016/j.joca.2011.12.003 (2012).

36 Kamekura, S. et al. Contribution of runt-related transcription factor 2 to the pathogenesis of osteoarthritis in mice after induction of knee joint instability. Arthritis Rheum 54, 2462-2470 (2006).

37 Kawaguchi, H. Endochondral ossification signals in cartilage degradation during osteoarthritis progression in experimental mouse models. Mol Cells 25, 1-6 (2008).

38 Saito, T. et al. Transcriptional regulation of endochondral ossification by HIF2alpha during skeletal growth and osteoarthritis development. Nat Med 16, 678686, doi:nm.2146 [pii] 10.1038/nm.2146 (2010).

39 Altman, R. D., Kates, J., Chun, L. E., Dean, D. D. \& Eyre, D. Preliminary observations of chondral abrasion in a canine model. Ann Rheum Dis 51, 1056-1062 (1992).

40 Furukawa, T., Eyre, D. R., Koide, S. \& Glimcher, M. J. Biochemical studies on 
repair cartilage resurfacing experimental defects in the rabbit knee. $J$ Bone Joint Surg Am 62, 79-89 (1980).

Mitchell, N. \& Shepard, N. The resurfacing of adult rabbit articular cartilage by multiple perforations through the subchondral bone. J Bone Joint Surg Am 58, 230-233 (1976).

Rae, P. J. \& Noble, J. Arthroscopic drilling of osteochondral lesions of the knee. $J$ Bone Joint Surg Br 71, 534 (1989).

43 Steinwachs, M. R., Guggi, T. \& Kreuz, P. C. Marrow stimulation techniques. Injury 39 Suppl 1, S26-31 (2008).

44 Emans, P. J. et al. Differential cell viability of chondrocytes and progenitor cells in tissue-engineered constructs following implantation into osteochondral defects. Tissue Eng 12, 1699-1709 (2006).

Emans, P. J., Surtel, D. A., Frings, E. J., Bulstra, S. K. \& Kuijer, R. In vivo generation of cartilage from periosteum. Tissue engineering 11, 369-377, doi:10.1089/ ten.2005.11.369 (2005).

Iwasaki, M. et al. Transforming growth factor-beta 1 stimulates chondrogenesis and inhibits osteogenesis in high density culture of periosteum-derived cells. Endocrinology 132, 1603-1608 (1993).

7 Nakahara, H., Bruder, S. P., Goldberg, V. M. \& Caplan, A. I. In vivo osteochondrogenic potential of cultured cells derived from the periosteum. Clin Orthop Relat Res, 223-232 (1990).

8 O'Driscoll, S. W., Recklies, A. D. \& Poole, A. R. Chondrogenesis in periosteal explants. An organ culture model for in vitro study. J Bone Joint Surg Am 76, 1042-1051 (1994).

Bouwmeester, S. J., Beckers, J. M., Kuijer, R., van der Linden, A. J. \& Bulstra, S. K. Long-term results of rib perichondrial grafts for repair of cartilage defects in the human knee. Int Orthop 21, 313-317 (1997).

0 Homminga, G. N., Bulstra, S. K., Bouwmeester, P. S. \& van der Linden, A. J. Perichondral grafting for cartilage lesions of the knee. J Bone Joint Surg Br 72, 1003-1007 (1990).

1 Homminga, G. N., Bulstra, S. K., Kuijer, R. \& van der Linden, A. J. Repair of sheep articular cartilage defects with a rabbit costal perichondrial graft. Acta Orthop Scand 62, 415-418 (1991).

2 O'Driscoll, S. W., Keeley, F. W. \& Salter, R. B. The chondrogenic potential of free autogenous periosteal grafts for biological resurfacing of major full-thickness defects in joint surfaces under the influence of continuous passive motion. An experimental investigation in the rabbit. J Bone Joint Surg Am 68, 1017-1035 (1986).

53 O'Driscoll, S. W., Keeley, F. W. \& Salter, R. B. Durability of regenerated articular cartilage produced by free autogenous periosteal grafts in major full-thickness defects in joint surfaces under the influence of continuous passive motion. A follow-up report at one year. J Bone Joint Surg Am 70, 595-606 (1988).

54 Skoog, T. \& Johansson, S. H. The formation of articular cartilage from free perichondrial grafts. Plast Reconstr Surg 57, 1-6 (1976).

55 Easley, M. E. \& Scranton, P. E., Jr. Osteochondral autologous transfer system. Foot Ankle Clin 8, 275-290 (2003).

56 Gross, A. E., McKee, N. H., Pritzker, K. P. \& Langer, F. Reconstruction of skeletal deficits at the knee. A comprehensive osteochondral transplant program. Clin Orthop Relat Res, 96-106 (1983).

57 Horas, U., Schnettler, R., Pelinkovic, D., Herr, G. \& Aigner, T. [Osteochondral transplantation versus autogenous chondrocyte transplantation. A prospective 
comparative clinical study]. Chirurg 71, 1090-1097 (2000).

58 Onstott, A. T., Moczo, A. \& Harris, N. L. Osteochondral autotransfer--newer treatment for chondral defects. Aorn J 71, 843-845, 848-851 (2000).

59 Czitrom, A. A., Keating, S. \& Gross, A. E. The viability of articular cartilage in fresh osteochondral allografts after clinical transplantation. J Bone Joint Surg Am 72, 574-581 (1990).

60 Garrett, J. C. Treatment of osteochondral defects of the distal femur with fresh osteochondral allografts: a preliminary report. Arthroscopy 2, 222-226 (1986).

61 Gross, A. E., Aubin, P., Cheah, H. K., Davis, A. M. \& Ghazavi, M. T. A fresh osteochondral allograft alternative. J Arthroplasty 17, 50-53 (2002).

62 Brittberg, M. Autologous chondrocyte implantation--technique and long-term follow-up. Injury 39 Suppl 1, S40-49, doi:S0020-1383(08)00062-4 [pii] 10.1016/j.injury.2008.01.040 (2008).

63 Brittberg, M. et al. Treatment of deep cartilage defects in the knee with autologous chondrocyte transplantation. N Engl J Med 331, 889-895 (1994).

Brittberg, M., Peterson, L., Sjogren-Jansson, E., Tallheden, T. \& Lindahl, A. Articular cartilage engineering with autologous chondrocyte transplantation. A review of recent developments. $J$ Bone Joint Surg Am 85-A Suppl 3, 109-115 (2003).

65 Bartlett, W. et al. Autologous chondrocyte implantation versus matrix-induced autologous chondrocyte implantation for osteochondral defects of the knee: a prospective, randomised study. J Bone Joint Surg Br 87, 640-645 (2005).

66 Harris, J. D. et al. Failures, re-operations, and complications after autologous chondrocyte implantation--a systematic review. Osteoarthritis and cartilage / OARS, Osteoarthritis Research Society 19, 779-791, doi:10.1016/ j.joca.2011.02.010 (2011).

67 Pietschmann, M. F. et al. The incidence and clinical relevance of graft hypertrophy after matrix-based autologous chondrocyte implantation. Am J Sports Med 40, 68-74, doi:10.1177/0363546511424396 (2012).

Nixon, A. J., Sams, A. E., Lust, G., Grande, D. \& Mohammed, H. O. Temporal matrix synthesis and histologic features of a chondrocyte-laden porous collagen cartilage analogue. Am J Vet Res 54, 349-356 (1993).

69 Sams, A. E. \& Nixon, A. J. Chondrocyte-laden collagen scaffolds for resurfacing extensive articular cartilage defects. Osteoarthritis Cartilage 3, 47-59 (1995).

70 Sams, A. E., Minor, R. R., Wootton, J. A., Mohammed, H. \& Nixon, A. J. Local and remote matrix responses to chondrocyte-laden collagen scaffold implantation in extensive articular cartilage defects. Osteoarthritis Cartilage 3, 61-70 (1995).

71 Wakitani, S. et al. Repair of rabbit articular surfaces with allograft chondrocytes embedded in collagen gel. J Bone Joint Surg Br 71, 74-80 (1989).

72 Sellers, R. S., Peluso, D. \& Morris, E. A. The effect of recombinant human bone morphogenetic protein-2 (rhBMP-2) on the healing of full-thickness defects of articular cartilage. J Bone Joint Surg Am 79, 1452-1463 (1997).

73 Sellers, R. S. et al. Repair of articular cartilage defects one year after treatment with recombinant human bone morphogenetic protein-2 (rhBMP-2). J Bone Joint Surg Am 82, 151-160 (2000).

74 Huang, Q., Goh, J. C., Hutmacher, D. W. \& Lee, E. H. In vivo mesenchymal cell recruitment by a scaffold loaded with transforming growth factor beta1 and the potential for in situ chondrogenesis. Tissue Eng 8, 469-482 (2002).

75 Macchiarini, P. et al. Clinical transplantation of a tissue-engineered airway. Lancet 372, 2023-2030, doi:S0140-6736(08)61598-6 [pii] $10.1016 / \mathrm{S} 0140-6736(08) 61598-6$ (2008). 

functional mammalian urinary bladder by tissue engineering. Nat Biotechnol 17, 149-155, doi:10.1038/6146 (1999).

Emans, P. J. et al. Autologous engineering of cartilage. Proc Natl Acad Sci U S A 107, 3418-3423, doi:0907774107 [pii]10.1073/pnas.0907774107 (2010). of osteochondral defects in rabbits with ectopically produced cartilage. Tissue engineering 11, 1789-1796, doi:10.1089/ten.2005.11.1789 (2005).

Abad, V. et al. The role of the resting zone in growth plate chondrogenesis. Endocrinology 143, 1851-1857 (2002).

Karlsson, C. \& Lindahl, A. Articular cartilage stem cell signalling. Arthritis research \& therapy 11, 121, doi:10.1186/ar2753 (2009).

Emans, P. J., Caron, M. M. J., van Rhijn, L. W., Shastri, V. P. \& Welting, T. J. M. Cartilage Tissue Engineering; Lessons Learned From Periosteum. Tissue Science \& Engineering S2:002, doi:doi:10.4172/2157-7552.S2-002 (2011).

Medvedev, S. P. et al. Human induced pluripotent stem cells derived from fetal neural stem cells successfully undergo directed differentiation into cartilage. Stem Cells Dev 20, 1099-1112, doi:10.1089/scd.2010.0249 (2011).

Barry, F. P. \& Murphy, J. M. Mesenchymal stem cells: clinical applications and biological characterization. Int J Biochem Cell Biol 36, 568-584, doi:10.1016/ j.biocel.2003.11.001 (2004).

84 Erlebacher, A., Filvaroff, E. H., Gitelman, S. E. \& Derynck, R. Toward a molecular understanding of skeletal development. Cell 80, 371-378 (1995).

Kronenberg, H. M. Developmental regulation of the growth plate. Nature 423, 332-336 (2003).

86 de Crombrugghe, B., Lefebvre, V. \& Nakashima, K. Regulatory mechanisms in the pathways of cartilage and bone formation. Curr Opin Cell Biol 13, 721-727 (2001).

87 Lefebvre, V., Behringer, R. R. \& de Crombrugghe, B. L-Sox5, Sox6 and Sox9 control essential steps of the chondrocyte differentiation pathway. Osteoarthritis Cartilage 9 Suppl A, S69-75 (2001).

Foster, J. W. et al. Campomelic dysplasia and autosomal sex reversal caused by mutations in an SRY-related gene. Nature 372, 525-530, doi:10.1038/372525a0 (1994).

89 Wagner, T. et al. Autosomal sex reversal and campomelic dysplasia are caused by mutations in and around the SRY-related gene SOX9. Cell 79, 1111-1120 (1994).

90 Akiyama, H., Chaboissier, M. C., Martin, J. F., Schedl, A. \& de Crombrugghe, B. The transcription factor Sox9 has essential roles in successive steps of the chondrocyte differentiation pathway and is required for expression of Sox 5 and Sox6. Genes Dev 16, 2813-2828 (2002).

91 Argentaro, A. et al. A SOX9 defect of calmodulin-dependent nuclear import in campomelic dysplasia/autosomal sex reversal. The Journal of biological chemistry 278, 33839-33847, doi:10.1074/jbc.M302078200 (2003).

92 Haudenschild, D. R., Chen, J., Pang, N., Lotz, M. K. \& D'Lima, D. D. Rho kinase -dependent activation of SOX9 in chondrocytes. Arthritis and rheumatism 62, 191 -200, doi:10.1002/art.25051 (2010).

93 Mertin, S., McDowall, S. G. \& Harley, V. R. The DNA-binding specificity of SOX9 and other SOX proteins. Nucleic acids research 27, 1359-1364 (1999).

94 Han, Y. \& Lefebvre, V. L-Sox5 and Sox6 drive expression of the aggrecan gene in cartilage by securing binding of Sox 9 to a far-upstream enhancer. Mol Cell Biol 28, 4999-5013, doi:MCB.00695-08 [pii] 
10.1128/MCB.00695-08 (2008).

95 Lefebvre, V., Huang, W., Harley, V. R., Goodfellow, P. N. \& de Crombrugghe, B. SOX9 is a potent activator of the chondrocyte-specific enhancer of the pro alpha1(II) collagen gene. Mol Cell Biol 17, 2336-2346 (1997).

96 Lefebvre, V., Li, P. \& de Crombrugghe, B. A new long form of Sox5 (L-Sox5), Sox 6 and Sox 9 are coexpressed in chondrogenesis and cooperatively activate the type II collagen gene. Embo $J$ 17, 5718-5733, doi:10.1093/emboj/17.19.5718 (1998).

97 Genzer, M. A. \& Bridgewater, L. C. A Col9a1 enhancer element activated by two interdependent SOX9 dimers. Nucleic acids research 35, 1178-1186, doi:10.1093/nar/gkm014 (2007).

98 Jenkins, E., Moss, J. B., Pace, J. M. \& Bridgewater, L. C. The new collagen gene COL27A1 contains SOX9-responsive enhancer elements. Matrix biology : journal of the International Society for Matrix Biology 24, 177-184, doi:10.1016/ j.matbio.2005.02.004 (2005).

99 Oh, C. D. et al. Identification of SOX9 interaction sites in the genome of chondrocytes. PLoS One 5, e10113, doi:10.1371/journal.pone.0010113 (2010).

100 Rentsendorj, O. et al. Highly conserved proximal promoter element harbouring paired Sox9-binding sites contributes to the tissue- and developmental stagespecific activity of the matrilin-1 gene. Biochem $J$ 389, 705-716, doi:10.1042/ BJ20050214 (2005).

101 Zeng, L., Kempf, H., Murtaugh, L. C., Sato, M. E. \& Lassar, A. B. Shh establishes an Nkx3.2/Sox9 autoregulatory loop that is maintained by BMP signals to induce somitic chondrogenesis. Genes Dev 16, 1990-2005, doi:10.1101/ gad.1008002 (2002).

102 Church, V. et al. Expression and function of Bapx1 during chick limb development. Anat Embryol (Berl) 209, 461-469, doi:10.1007/s00429-005-0464-z (2005).

103 Provot, S. et al. Nkx3.2/Bapx1 acts as a negative regulator of chondrocyte maturation. Development 133, 651-662, doi:10.1242/dev.02258 (2006).

104 Yamashita, S. et al. Sox9 directly promotes Bapx1 gene expression to repress Runx2 in chondrocytes. Experimental cell research 315, 2231-2240, doi:10.1016/ j.yexcr.2009.03.008 (2009).

105 Long, F., Schipani, E., Asahara, H., Kronenberg, H. \& Montminy, M. The CREB family of activators is required for endochondral bone development. Development 128, 541-550 (2001).

106 Wang, Z. Q. et al. Bone and haematopoietic defects in mice lacking c-fos. Nature 360, 741-745, doi:10.1038/360741a0 (1992).

107 Hunziker, E. B. Mechanism of longitudinal bone growth and its regulation by growth plate chondrocytes. Microsc Res Tech 28, 505-519, doi:10.1002/ jemt.1070280606 (1994).

108 Arnold, M. A. et al. MEF2C transcription factor controls chondrocyte hypertrophy and bone development. Dev Cell 12, 377-389, doi:S1534-5807(07)00054-8 [pii]

10.1016/j.devcel.2007.02.004 (2007).

109 Drissi, M. H. et al. Runx2/Cbfa1 stimulation by retinoic acid is potentiated by BMP2 signaling through interaction with Smad1 on the collagen X promoter in chondrocytes. Journal of cellular biochemistry 90, 1287-1298 (2003).

110 Linsenmayer, T. F., Eavey, R. D. \& Schmid, T. M. Type X collagen: a hypertrophic cartilage-specific molecule. Pathol Immunopathol Res 7, 14-19 (1988).

111 Zheng, Q. et al. Type X collagen gene regulation by Runx2 contributes directly to 
its hypertrophic chondrocyte-specific expression in vivo. J Cell Biol 162, 833842, doi: $10.1083 /$ jcb.200211089

jcb.200211089 [pii] (2003).

112 Inada, M. et al. Maturational disturbance of chondrocytes in Cbfa1-deficient mice. Developmental dynamics : an official publication of the American Association of Anatomists 214, 279-290, doi:10.1002/(SICI)1097-0177(199904) 214:4<279::AID-AJA1>3.0.CO;2-W (1999).

113 Komori, T. et al. Targeted disruption of Cbfa1 results in a complete lack of bone formation owing to maturational arrest of osteoblasts. Cell 89, 755-764 (1997).

114 Otto, F. et al. Cbfa1, a candidate gene for cleidocranial dysplasia syndrome, is essential for osteoblast differentiation and bone development. Cell 89, 765-771 (1997).

115 Takeda, S., Bonnamy, J. P., Owen, M. J., Ducy, P. \& Karsenty, G. Continuous expression of Cbfa1 in nonhypertrophic chondrocytes uncovers its ability to induce hypertrophic chondrocyte differentiation and partially rescues Cbfa1deficient mice. Genes Dev 15, 467-481, doi:10.1101/gad.845101 (2001).

116 Ueta, C. et al. Skeletal malformations caused by overexpression of Cbfa1 or its dominant negative form in chondrocytes. J Cell Biol 153, 87-100 (2001).

117 Gerber, H. P. et al. VEGF couples hypertrophic cartilage remodeling, ossification and angiogenesis during endochondral bone formation. Nat Med 5, 623-628 (1999).

118 Hess, J., Porte, D., Munz, C. \& Angel, P. AP-1 and Cbfa/runt physically interact and regulate parathyroid hormone-dependent MMP13 expression in osteoblasts through a new osteoblast-specific element 2/AP-1 composite element. The Journal of biological chemistry 276, 20029-20038, doi:10.1074/jbc.M010601200 (2001).

119 Sato, M. et al. Transcriptional regulation of osteopontin gene in vivo by PEBP2alphaA/CBFA1 and ETS1 in the skeletal tissues. Oncogene 17, 15171525, doi:10.1038/sj.onc.1202064 (1998).

120 Beier, F. et al. TGFbeta and PTHrP control chondrocyte proliferation by activating cyclin D1 expression. Mol Biol Cell 12, 3852-3863 (2001).

121 Zhang, M. et al. PTHrP prevents chondrocyte premature hypertrophy by inducing cyclin-D1-dependent Runx2 and Runx3 phosphorylation, ubiquitylation and proteasomal degradation. Journal of cell science 122, 1382-1389, doi:10.1242/ jes.040709 (2009).

122 St-Jacques, B., Hammerschmidt, M. \& McMahon, A. P. Indian hedgehog signaling regulates proliferation and differentiation of chondrocytes and is essential for bone formation. Genes Dev 13, 2072-2086 (1999).

123 Bitgood, M. J. \& McMahon, A. P. Hedgehog and Bmp genes are coexpressed at many diverse sites of cell-cell interaction in the mouse embryo. Dev Biol 172, 126 -138, doi:10.1006/dbio.1995.0010 (1995).

124 Long, F., Zhang, X. M., Karp, S., Yang, Y. \& McMahon, A. P. Genetic manipulation of hedgehog signaling in the endochondral skeleton reveals a direct role in the regulation of chondrocyte proliferation. Development 128, 5099-5108 (2001).

125 Vortkamp, A. et al. Regulation of rate of cartilage differentiation by Indian hedgehog and PTH-related protein. Science 273, 613-622 (1996).

126 Yoon, B. S. et al. BMPs regulate multiple aspects of growth-plate chondrogenesis through opposing actions on FGF pathways. Development 133, 4667-4678, doi:dev.02680 [pii] 10.1242/dev.02680 (2006).

127 Yoon, B. S. \& Lyons, K. M. Multiple functions of BMPs in chondrogenesis. 
Journal of cellular biochemistry 93, 93-103 (2004).

128 Urist, M. R. Bone: formation by autoinduction. Science 150, 893-899 (1965).

129 Chimal-Monroy, J., Bravo-Ruiz, M. T. \& Diaz de Leon, L. Regulation of chondrocyte differentiation by transforming growth factors beta 1 , beta 2 , beta 3 , and beta 5. Ann N Y Acad Sci 785, 241-244 (1996).

130 Chimal-Monroy, J. \& Diaz de Leon, L. Differential effects of transforming growth factors beta 1 , beta 2 , beta 3 and beta 5 on chondrogenesis in mouse limb bud mesenchymal cells. Int J Dev Biol 41, 91-102 (1997).

131 Ferguson, C. M. et al. Transforming growth factor-beta1 induced alteration of skeletal morphogenesis in vivo. J Orthop Res 22, 687-696, doi:10.1016/ j.orthres.2003.10.023 (2004).

132 Ferguson, C. M. et al. Smad2 and 3 mediate transforming growth factor-beta1induced inhibition of chondrocyte maturation. Endocrinology 141, 4728-4735 (2000).

133 Johnstone, B., Hering, T. M., Caplan, A. I., Goldberg, V. M. \& Yoo, J. U. In vitro chondrogenesis of bone marrow-derived mesenchymal progenitor cells. Experimental cell research 238, 265-272, doi:10.1006/excr.1997.3858 (1998).

134 Lorda-Diez, C. I., Montero, J. A., Martinez-Cue, C., Garcia-Porrero, J. A. \& Hurle, J. M. Transforming growth factors beta coordinate cartilage and tendon differentiation in the developing limb mesenchyme. The Journal of biological chemistry 284, 29988-29996, doi:10.1074/jbc.M109.014811 (2009).

135 Feng, X. H., Zhang, Y., Wu, R. Y. \& Derynck, R. The tumor suppressor Smad4/ DPC4 and transcriptional adaptor $\mathrm{CBP} / \mathrm{p} 300$ are coactivators for smad3 in TGFbeta-induced transcriptional activation. Genes Dev 12, 2153-2163 (1998).

136 Lagna, G., Hata, A., Hemmati-Brivanlou, A. \& Massague, J. Partnership between DPC4 and SMAD proteins in TGF-beta signalling pathways. Nature 383, 832836, doi:10.1038/383832a0 (1996).

137 Elford, P. R. et al. Induction of swelling, synovial hyperplasia and cartilage proteoglycan loss upon intra-articular injection of transforming growth factor beta-2 in the rabbit. Cytokine 4, 232-238 (1992).

138 van Beuningen, H. M., van der Kraan, P. M., Arntz, O. J. \& van den Berg, W. B. Does TGF-beta protect articular cartilage in vivo? Agents Actions Suppl 39, $127-$ 131 (1993).

139 van Beuningen, H. M., van der Kraan, P. M., Arntz, O. J. \& van den Berg, W. B. Transforming growth factor-beta 1 stimulates articular chondrocyte proteoglycan synthesis and induces osteophyte formation in the murine knee joint. Lab Invest 71, 279-290 (1994).

140 Hunziker, E. B. Growth-factor-induced healing of partial-thickness defects in adult articular cartilage. Osteoarthritis Cartilage 9, 22-32 (2001).

141 Aberle, H., Bauer, A., Stappert, J., Kispert, A. \& Kemler, R. beta-catenin is a target for the ubiquitin-proteasome pathway. Embo J 16, 3797-3804, doi:10.1093/ emboj/16.13.3797 (1997).

142 Behrens, J. et al. Functional interaction of beta-catenin with the transcription factor LEF-1. Nature 382, 638-642, doi:10.1038/382638a0 (1996).

143 Reya, T. \& Clevers, H. Wnt signalling in stem cells and cancer. Nature 434, 843850, doi:10.1038/nature03319 (2005).

144 Day, T. F., Guo, X., Garrett-Beal, L. \& Yang, Y. Wnt/beta-catenin signaling in mesenchymal progenitors controls osteoblast and chondrocyte differentiation during vertebrate skeletogenesis. Dev Cell 8, 739-750, doi:10.1016/ j.devcel.2005.03.016 (2005).

145 Dong, Y. F., Soung do, Y., Schwarz, E. M., O'Keefe, R. J. \& Drissi, H. Wnt in- 
duction of chondrocyte hypertrophy through the Runx2 transcription factor. $J$ Cell Physiol 208, 77-86, doi:10.1002/jcp.20656 (2006).

146 Hill, T. P., Spater, D., Taketo, M. M., Birchmeier, W. \& Hartmann, C. Canonical Wnt/beta-catenin signaling prevents osteoblasts from differentiating into chondrocytes. Dev Cell 8, 727-738, doi:10.1016/j.devcel.2005.02.013 (2005).

147 Akiyama, H. Control of chondrogenesis by the transcription factor Sox9. Mod Rheumatol 18, 213-219, doi:10.1007/s10165-008-0048-x (2008).

148 Akiyama, H. et al. Essential role of Sox9 in the pathway that controls formation of cardiac valves and septa. Proceedings of the National Academy of Sciences of the United States of America 101, 6502-6507, doi:10.1073/pnas.0401711101 (2004).

149 Ryu, J. H. et al. Regulation of the chondrocyte phenotype by beta-catenin. Development 129, 5541-5550 (2002).

150 Topol, L., Chen, W., Song, H., Day, T. F. \& Yang, Y. Sox9 inhibits Wnt signaling by promoting beta-catenin phosphorylation in the nucleus. The Journal of biological chemistry 284, 3323-3333, doi:10.1074/jbc.M808048200 (2009).

151 Yang, K. G., Saris, D. B., Verbout, A. J., Creemers, L. B. \& Dhert, W. J. The effect of synovial fluid from injured knee joints on in vitro chondrogenesis. Tissue Eng 12, 2957-2964 (2006).

152 Saris, D. B., Dhert, W. J. \& Verbout, A. J. Joint homeostasis. The discrepancy between old and fresh defects in cartilage repair. J Bone Joint Surg Br 85, 10671076 (2003).

153 Richter, W. Mesenchymal stem cells and cartilage in situ regeneration. Journal of internal medicine 266, 390-405 (2009).

154 Einhorn, T. A. The science of fracture healing. Journal of orthopaedic trauma 19, S4-6 (2005).

155 Gerstenfeld, L. C., Cullinane, D. M., Barnes, G. L., Graves, D. T. \& Einhorn, T. A. Fracture healing as a post-natal developmental process: molecular, spatial, and temporal aspects of its regulation. Journal of cellular biochemistry 88, 873-884 (2003).

156 Grundnes, O. \& Reikeras, O. The importance of the hematoma for fracture healing in rats. Acta Orthop Scand 64, 340-342 (1993).

157 Lieberman, J. R., Daluiski, A. \& Einhorn, T. A. The role of growth factors in the repair of bone. Biology and clinical applications. J Bone Joint Surg Am 84-A, 1032-1044 (2002).

158 Mountziaris, P. M. \& Mikos, A. G. Modulation of the Inflammatory Response for Enhanced Bone Tissue Regeneration. Tissue Eng Part B Rev (2008).

159 Einhorn, T. A., Majeska, R. J., Rush, E. B., Levine, P. M. \& Horowitz, M. C. The expression of cytokine activity by fracture callus. $J$ Bone Miner Res 10, 12721281, doi:10.1002/jbmr.5650100818 (1995).

160 Rundle, C. H. et al. Microarray analysis of gene expression during the inflammation and endochondral bone formation stages of rat femur fracture repair. Bone 38, 521-529 (2006).

161 Gerstenfeld, L. C. et al. Impaired intramembranous bone formation during bone repair in the absence of tumor necrosis factor-alpha signaling. Cells Tissues Organs 169, 285-294 (2001).

162 Gerstenfeld, L. C. et al. Impaired fracture healing in the absence of TNF-alpha signaling: the role of TNF-alpha in endochondral cartilage resorption. $J$ Bone Miner Res 18, 1584-1592 (2003).

163 Franchimont, N., Rydziel, S. \& Canalis, E. Interleukin 6 is autoregulated by transcriptional mechanisms in cultures of rat osteoblastic cells. J Clin Invest 100, 
1797-1803 (1997).

164 Kudo, O. et al. Interleukin-6 and interleukin-11 support human osteoclast formation by a RANKL-independent mechanism. Bone 32, 1-7 (2003).

165 Brochhausen, C., Neuland, P., Kirkpatrick, C. J., Nusing, R. M. \& Klaus, G. Cyclooxygenases and prostaglandin E2 receptors in growth plate chondrocytes in vitro and in situ--prostaglandin E2 dependent proliferation of growth plate chondrocytes. Arthritis Res Ther 8, R78 (2006).

166 Naik, A. A. et al. Reduced COX-2 expression in aged mice is associated with impaired fracture healing. J Bone Miner Res 24, 251-264 (2009).

167 Simon, A. M. \& O'Connor, J. P. Dose and time-dependent effects of cyclooxygenase-2 inhibition on fracture-healing. J Bone Joint Surg Am 89, 500-511 (2007).

168 Zhang, X. et al. Cyclooxygenase-2 regulates mesenchymal cell differentiation into the osteoblast lineage and is critically involved in bone repair. J Clin Invest 109, 1405-1415 (2002).

169 Diwan, A. D., Wang, M. X., Jang, D., Zhu, W. \& Murrell, G. A. Nitric oxide modulates fracture healing. J Bone Miner Res 15, 342-351 (2000).

170 Arasapam, G., Scherer, M., Cool, J. C., Foster, B. K. \& Xian, C. J. Roles of COX2 and iNOS in the bony repair of the injured growth plate cartilage. Journal of cellular biochemistry 99, 450-461 (2006).

171 Baldik, Y. et al. Deletion of iNOS gene impairs mouse fracture healing. Bone 37, 32-36 (2005).

172 Zhu, W., Murrell, G. A., Lin, J., Gardiner, E. M. \& Diwan, A. D. Localization of nitric oxide synthases during fracture healing. J Bone Miner Res 17, 1470-1477 (2002).

173 Croitoru-Lamoury, J., Lamoury, F. M., Zaunders, J. J., Veas, L. A. \& Brew, B. J. Human mesenchymal stem cells constitutively express chemokines and chemokine receptors that can be upregulated by cytokines, IFN-beta, and Copaxone. $J$ Interferon Cytokine Res 27, 53-64, doi:10.1089/jir.2007.0037 (2007).

174 Gilmore, T. D. \& Herscovitch, M. Inhibitors of NF-kappaB signaling: 785 and counting. Oncogene 25, 6887-6899 (2006).

175 Hayden, M. S. \& Ghosh, S. Shared principles in NF-kappaB signaling. Cell 132, 344-362 (2008).

176 Pevsner-Fischer, M. et al. Toll-like receptors and their ligands control mesenchymal stem cell functions. Blood 109, 1422-1432, doi:10.1182/blood-2006-06028704 (2007).

177 Grau, A. M., Datta, P. K., Zi, J., Halder, S. K. \& Beauchamp, R. D. Role of Smad proteins in the regulation of NF-kappaB by TGF-beta in colon cancer cells. Cell Signal 18, 1041-1050, doi:S0898-6568(05)00235-4 [pii] 10.1016/j.cellsig.2005.08.021 (2006).

178 Wu, S., Fadoju, D., Rezvani, G. \& De Luca, F. Stimulatory effects of insulin-like growth factor-I on growth plate chondrogenesis are mediated by nuclear factorkappaB p65. J Biol Chem 283, 34037-34044 (2008).

179 Franzoso, G. et al. Requirement for NF-kappaB in osteoclast and B-cell development. Genes Dev 11, 3482-3496 (1997).

180 OARSI. World Congress on Osteoarthritis (Montreal,Quebec, Canada). Osteoarthritis Cartilage 17 (2009).

181 Ushita, M. et al. Transcriptional induction of SOX9 by NF-kappaB family member RelA in chondrogenic cells. Osteoarthritis Cartilage (2009).

182 Feng, J. Q. et al. NF-kappaB specifically activates BMP-2 gene expression in growth plate chondrocytes in vivo and in a chondrocyte cell line in vitro. $J$ Biol 
Chem 278, 29130-29135 (2003).

183 Fukui, N. et al. Pro-inflammatory cytokine tumor necrosis factor-alpha induces bone morphogenetic protein-2 in chondrocytes via mRNA stabilization and transcriptional up-regulation. J Biol Chem 281, 27229-27241 (2006).

184 Fukui, N., Zhu, Y., Maloney, W. J., Clohisy, J. \& Sandell, L. J. Stimulation of BMP-2 expression by pro-inflammatory cytokines IL-1 and TNF-alpha in normal and osteoarthritic chondrocytes. J Bone Joint Surg Am 85-A Suppl 3, 59-66 (2003).

185 van der Windt, A. E. et al. Physiological tonicity improves human chondrogenic marker expression through nuclear factor of activated T-cells 5 in vitro. Arthritis research \& therapy 12, R100, doi:10.1186/ar3031 (2010).

186 Caron, M. M. et al. Activation of NF-kappaB/p65 Facilitates Early Chondrogenic Differentiation during Endochondral Ossification. PLoS One 7, e33467, doi:10.1371/journal.pone.0033467 (2012). 



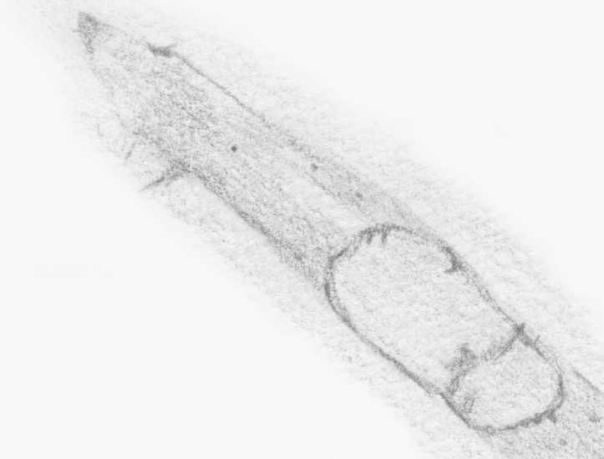

Chapter 2

Activation of NF-kappa B/p65 Facilitates Early

Chondrogenic Differentiation during Endochondral Ossification

Adapted from:

Activation of $N F-\kappa B / p 65$ Facilitates Early Chondrogenic Differentiation during Endochondral Ossification

Marjolein M.J. Caron ${ }^{1}$, Pieter J.Emans ${ }^{1}$, Don A.M. Surtel ${ }^{1}$, Andy Cremers ${ }^{1}$, Jan-Willem Voncken ${ }^{2}$, Tim J. M.

Welting $^{1^{*}}$ and Lodewijk W. van Rhijn ${ }^{1 *}$

${ }^{1}$ Department of Orthopaedic Surgery, Maastricht University Medical Center, Maastricht, the Netherlands

${ }^{2}$ Department of Molecular Genetics, Maastricht University Medical Center, Maastricht, the Netherlands

*Contributed equally

PLoS ONE. (2012); 7(3):e33467 


\section{Alestract}

Introduction: $\mathrm{NF}-\mathrm{kB} / \mathrm{p} 65$ has been reported to be involved in regulation of chondrogenic differentiation. However, its function in relation to key chondrogenic factor Sox 9 and onset of chondrogenesis during endochondral ossification is poorly understood. We hypothesized that the early onset of chondrogenic differentiation is initiated by transient $\mathrm{NF}-\kappa \mathrm{B} / \mathrm{p} 65$ signaling.

Methodology: The role of NF-kB/p65 in early chondrogenesis was investigated in different in vitro, ex vivo and in vivo endochondral models: ATDC5 cells, hBMSCs, chicken periosteal explants and growth plates of six weeks old mice. NF- $\mathrm{BB} / \mathrm{p} 65$ activation was manipulated using pharmacological inhibitors, RNAi and activating agents. Gene expression and protein expression analysis, and (immuno)histochemical stainings were employed to determine the role of $\mathrm{NF}-\mathrm{\kappa B} / \mathrm{p} 65$ in the chondrogenic phase of endochondral development.

Results: Our data show that chondrogenic differentiation is facilitated by early transient activation of $\mathrm{NF}-\kappa \mathrm{B} / \mathrm{p} 65$. NF- $\mathrm{\kappa B} / \mathrm{p} 65$-mediated signaling determines early expression of Sox9 and facilitates the subsequent chondrogenic differentiation programming by signaling through key chondrogenic pathways.

Conclusions: The presented data demonstrate that NF-KB/p65 signaling, as well as its intensity and timing, represents one of the transcriptional regulatory mechanisms of the chondrogenic developmental program of chondroprogenitor cells during endochondral ossification. Importantly, these results provide novel possibilities to improve the success of cartilage and bone regenerative techniques. 


\section{Introduction}

Chondrogenic differentiation encompasses the commitment and differentiation of chondroprogenitor cells to chondrocytes. In addition to providing articulating joint surfaces with functional cartilage and maintaining cartilage integrity, chondrogenic differentiation plays an essential role during endochondral ossification. Skeletal growth and bone fracture healing depend on endochondral ossification; growth plate chondrocytes or fracture callus chondrocytes originating from mesenchymal progenitors gradually differentiate into mineralized hypertrophic chondrocytes and finally die by apoptosis. The remaining mineralized extracellular matrix provides a molecular scaffold for infiltrating osteoblasts and osteoclasts to adhere to and remodel, setting the stage for de novo bone deposition ${ }^{1-3}$. Transcriptional targets of NF-KB (nuclear factor kappa-light-chain-enhancer of activated B cells) have been recognized as key developmental signaling mediators that regulate endochondral ossification. Early bone fracture healing by endochondral ossification depends on a haematoma-induced inflammatory environment ${ }^{4}$ and several NF-KB-target genes (e.g. interleukin (Il)-6, tumor necrosis factor alpha (TNF $\alpha)$, cyclooxygenase (COX)2 and inducible nitric oxide synthase (iNOS)) are involved in bone fracture repair ${ }^{5,6}$. Besides its functions in transcriptional regulation of general catabolic inflammatory processes, $\mathrm{NF}-\mathrm{kB}$ has been linked to skeletal development ${ }^{7}$. Double knockout (KO) of NF- $\mathrm{B}$ subunits p50 and p52 shows abnormal skeletal development in mice, which was attributed to impaired growth plate function ${ }^{8}$. Recently, NF-кB subunit RelA (V-rel reticuloendotheliosis viral oncogene homolog $\mathrm{A}$ or p65) was reported to be activated by Nkx3.2/Bapx1 (NK3 homeobox 2/Bagpipe homeobox homolog1) to control chondrocyte viability ${ }^{9}$. Moreover, RelA was identified as a transcription factor for bone morphogenic protein (BMP) $2^{8,10}$ and Sox9 (SRY (sex determining region Y)-box 9) in mature chondrocytes during endochondral ossification ${ }^{11}$. Sox 9 is expressed by chondroprogenitor cells and is indispensable for chondrogenic differentiation ${ }^{12-14}$. Sox 9 drives the expression of cartilage matrix genes Collagen type II (Col2a1) and Aggrecan (Acan) cooperatively with L-Sox 5 and Sox $6^{15-17}$ and as such maintains chondrocyte phenotype. The involvement NF-KB/p65 as indispensable factor during chondrogenic development has been studied in the context of mature chondrocytes. However, the mechanism by which NF-KB/p65 signaling influences early differentiation of chondroprogenitors remains elusive. We hypothesized that the initiation of chondrogenic differentiation is regulated by transient NF-KB/ p65 signaling. 
Our data show that during the very first hours of chondroprogenitor differentiation a transient activation of NF-kB/p65 occurs which, in part, regulates the transient expression of key chondrogenic controller Sox9 at the early phase of chondrogenesis. This early transient Sox9 induction precedes the induction of Sox 9 that is described to be related to late cartilage matrix synthesis ${ }^{15,16}$, revealing a novel bi-phasic induction for Sox9 during chondrogenic differentiation. We found indications that through the early Sox9 induction the transient NF-KB/p65 activation determines, at least in part, the late stage fate of the chondrogenic differentiation process. Inhibition of $\mathrm{NF}-\mathrm{kB} / \mathrm{p} 65$ mediated signaling is accompanied by inhibition of early Sox9 expression and subsequent inhibition of late stage chondrogenesis. In line with these findings, brief early NF- $\kappa \mathrm{B}$ stimulation using different NF- $\kappa \mathrm{B}$ activating molecules (lipopolysaccharide (LPS), tumor necrosis factor $\alpha(\mathrm{TNF} \alpha)$ or BMP-2), enhanced chondrogenesis in our in vitro and ex vivo endochondral models. Our findings demonstrate that NF-KB/p65 signaling, as well as its intensity and timing, is an important factor in the regulation of the early chondrogenic developmental program of chondroprogenitor cells and thereby in part determines endochondral ossification.

\section{Materials and Methods}

\subsection{ATDC5 cell culture}

ATDC5 cells ${ }^{18}$ were cultured in proliferation medium (DMEM(Dulbecco's modified eagle medium)/F12 (Invitrogen, Carlsbad, CA, USA), 5\% fetal calf serum (FCS) (PAA, Pasching, Austria), 1\% antibiotic/antimycotic (Invitrogen) and 1\% NEAA (non-essential amino acids) (Invitrogen)). Differentiation medium comprised proliferation medium supplemented with $10 \mu \mathrm{g} / \mathrm{ml}$ insulin (Sigma, St. Louis, MO, USA), $10 \mu \mathrm{g} / \mathrm{ml}$ transferrin (Roche Applied Science) and $30 \mathrm{nM}$ sodium selenite (Sigma). Cells were plated at 6,400 cells $/ \mathrm{cm}^{2}$ and the following day chondrogenesis was initiated by changing the proliferation medium to differentiation medium (or proliferation medium in case of data presented in Figure 2.7). Medium was changed every two days and every day from day ten onwards. To inhibit NF-KB, TLCK (Acros), Parthenolide (Sigma) and Sulfasalazine (Sigma) were used. LPS (lipopolysaccharide) (Sigma) or TNF $\alpha$ (R\&D) were used as NF- $\mathrm{B} / \mathrm{p} 65$ activators. BMP-2 was used at $30 \mathrm{ng} / \mathrm{ml}$ (Sigma). For RNAi-experiments a p65 small interfering RNA (siRNA) duplex (sense: 5'-AGAGGACAUUGAGGUGUAUTT-3', anti-sense: 5'- 
AUACACCUCAAUGUCCUCUTT-3'), a Sox9 siRNA duplex (sense: 5' GACUCACAUCUCUCCUAAUTT-3', anti-sense: 5'- AUUAGGAGAGAUGUGAGUCTT-3') and a scrambled siRNA-duplex (indicated by "Control") were used (Eurogentec, Seraing, Belgium). ATDC5 cells were seeded at 25,000 cells $/ \mathrm{cm}^{2}$ and transfection with siRNAs (100 $\mathrm{nM}$ for p65 and $50 \mathrm{nM}$ for Sox9) was performed using ICAfectin 442 (Eurogentec) according to manufacturers' protocol. Cells were cultured for two days before chondrogenesis was initiated.

\section{2 hBMSC isolation and culture}

Human bone marrow stem cells (hBMSCs) were obtained from residual iliac crest bone marrow aspirate from young, genetically healthy individuals undergoing spinal surgery. The Maastricht University Medical Centre institutional policy on the use of residual human surgical material specifically states that no informed consent is needed in the case of residual surgical material. However an approval from the institutional Medical Ethical Committee (MEC) for the use of this material is required. The MEC approved this study and assigned approval ID: MEC 08-4-056. Human BMSCs were isolated from the aspirate using Ficoll Paque (Amersham Pharmacia, Diegem, Belgium). Proliferation medium consisted of DMEM high-glucose (Invitrogen,), 10\% FCS (ES-grade), 1\% antibiotic/ antimycotic and $1 \%$ NEAA. Passage 5 cells were plated at 30,000 cells $/ \mathrm{cm}^{2}$ and chondrogenesis was initiated the next day by changing to differentiation medium (proliferation medium supplemented with $1 \%$ ITS (insulin-transferrin-sodium selenite media supplement) (Invitrogen), $50 \mu \mathrm{g} / \mathrm{ml}_{\mathrm{L}}$-ascorbic acid-2-phosphate (Sigma) and $1 \mathrm{ng} / \mathrm{ml}$ transforming growth factor $\beta 3$ (TGF- $\beta 3)$ (R\&D, Minneapolis, MN, USA) $)^{19}$. In experiments for Figure 2.7B the proliferation medium was changed with proliferation medium. Medium was changed every two days. TLCK (Acros, Geel, Belgium) was used to inhibit NF- $\kappa$ B/p65 activation and LPS (Sigma) was used as NF- $\mathrm{B} / \mathrm{p} 65$ activator.

\subsection{Chicken periosteum agarose culture (cPAC)}

Fertilized eggs of Dekalb white chickens ('t Anker, Ochten, the Netherlands) were placed in a polyhatch incubator (Brisnea, Sandford, UK) at $39.2^{\circ} \mathrm{C}$ and at relative humidity of $40 \%$. At embryonic day 16 , embryos were removed and sacrificed by rapid decapitation. Incubation period corresponded to embyos at Hamburger and Hamilton stage 42. Periosteum was dissected from tibiae using aseptic techniques. Periostea were embedded in $1 \%$ low-melting agarose $/ 0.9 \% \mathrm{NaCl}$ using procedures described before ${ }^{20,21}$. Proliferation 
medium (DMEM/F12, 10\% FCS, 1\% antibiotic/antimycotic, 1\% NEAA) was added and incubated overnight at $37^{\circ} \mathrm{C} / 5 \% \mathrm{CO}_{2}$. The next day, medium was changed with proliferation medium supplemented with or without LPS (Sigma). For the differentiation control, medium was changed to differentiation medium (proliferation medium supplemented with 1\% ITS (Invitrogen), $50 \mu \mathrm{g} / \mathrm{ml}_{\mathrm{L}}$-ascorbic acid-2-phosphate (Sigma), $10 \mathrm{ng} / \mathrm{ml}$ TGF- $\beta 3$ (R\&D)). Medium was changed every two days. Ethical approval by the institutional animal ethical committee was waived for these experiments as institutional regulations state that no approval from an animal ethical committee is needed to perform embryonic chicken experiments.

\subsection{Mouse growth plates}

The growth plates were isolated from tibias of six weeks old C57BL/6 mice. These were surplus wildtype mice from another unrelated experiment. This experiment was approved by the Maastricht University animal ethical committee (DEC) and assigned approval ID: DEC 2008-042. The tibia's were isolated and fixed in formalin. The growth plates were separated from the rest of the tibia and decalcified in 0.5 M EDTA pH 7.8 for two weeks. EDTA was refreshed every two days. Growth plates were dehydrated and embedded in paraffin. Five micrometer sections were cut and positioned on Superfrost Plus slides for immunohistochemistry (IHC).

\section{$\underline{2.5 R T-q P C R}$}

Total RNA was extracted with TRIzol (Invitrogen). Quantity and purity of extracted RNA were determined by UV-spectrometry (Nanodrop, Thermo Scientific, the Netherlands). DNA-free total RNA was reverse transcribed to cDNA using standard procedures and random hexamer priming. Real time quantitative PCR (RT-qPCR) was performed using Mesagreen qPCR mastermix plus for SYBR Green (Eurogentec) and an Applied Biosystems ABI PRISM 7300 Sequence Detection System for amplification with the following profile: initial denaturation 10 minutes at $95^{\circ} \mathrm{C}$, followed by 40 cycles of amplification (15 seconds at $95^{\circ} \mathrm{C}$ and 1 minute at $60^{\circ} \mathrm{C}$ ), followed by a dissociation curve. Data were analyzed using the standard curve method and relative quantification of mRNA expression was normalized to a housekeeping mRNA. Primer sequences are depicted in Table 2.1.

\section{$\underline{2.6 \text { Immunoblotting }}$}

Cells were lysed in RIPA buffer $(150 \mathrm{mM} \mathrm{NaCl}, 1 \% \mathrm{NP}-40,0.5 \%$ Sodium deoxycho- 
late, $0.1 \%$ SDS, $50 \mathrm{mM}$ Tris $\mathrm{pH}$ 8.0, $5.0 \mathrm{mM}$ EDTA $\mathrm{pH}$ 8.0, $0.5 \mathrm{mM}$ dithiothreitol (DTT) and $1 \mathrm{mM}$ phenylmethylsulfonylfluoride (PMSF). Nuclear extracts were prepared by lysing cells in a buffer containing $20 \mathrm{mM}$ HEPES (pH 7.8), $20 \mathrm{mM} \mathrm{KCl}, 4 \mathrm{mM} \mathrm{MgCl} 2,0.2$ mM EDTA (pH 8.0), $1 \mathrm{mM}$ DTT, $0.2 \mathrm{mM}$ sodiumvanadate, $0.4 \mathrm{mM}$ PMSF, $0.3 \mathrm{mg} / \mathrm{ml}$ leupeptin and $0.2 \mathrm{mM}$ sodiumfluoride). Nuclei were separated from cytoplasm by centrifugation $(16.000 \mathrm{x} \mathrm{g})$ and the nuclear pellet was lysed in RIPA buffer. Extracts were sonicated and protein concentrations were determined using the BCA method (Sigma). Polypeptides were separated by SDS-PAGE and transferred to nitrocellulose membranes by electroblotting. Primary antibodies for immunodetection were anti-Col2a1 (Southern Biotech, Birmingham, AL, USA), anti-Col10a1 (Calbiochem, Darmstadt, Germany), antiSox9 (Abcam, Cambridge, UK), anti-COX-2 (Cayman, Ann Arbor, US), anti-iNOS (Abcam), anti-p65 (Santa Cruz Biotechnologies, CA, USA), anti-Runx2 (MBL, Woburn, MA, USA) and anti- $\alpha$-Tubulin (Sigma). Bound primary antibodies were detected with immunoglobulins conjugated with HRP (DakoCytomation, Glostrup, Denmark) and visualized by enhanced chemoluminescence (ECL). ECL signals were quantified using Biorad Quantity One 4.6.7 software and relative differences, corrected for background and housekeeper, were determined as compared to control conditions or $\mathrm{t}=0$.

\section{7 (Immuno)histochemistry}

Chicken PAC samples were dehydrated following standard procedures and embedded in paraffin. Tissue sections were cut at $5 \mu \mathrm{m}$, deparaffinized and rehydrated using standard protocols. Proteoglycans were stained with Safranin-O (0.1\%) (Sigma) and counterstained with Fast Green (0.1\%) (Sigma). Stained sections were dehydrated and mounted in Histomount (Thermo Shandon). For Sox9, p65, iNOS and COX-2 expression in 6-weeks old mouse growth plate and cPAC, sections were deparaffinized and antigen retrieval was performed by incubation in boiling citrate buffer $(1.8 \mathrm{mM}$ citric acid and $8.2 \mathrm{mM}$ tri-sodium citrate) for 30 minutes. For Col2al detection in $\mathrm{cPAC}$, sections were digested with $0.4 \%$ hyaluronidase (Sigma) for 30 minutes at $37^{\circ} \mathrm{C}$. Endogenous peroxidase activity was inactivated and sections were blocked with $10 \%$ normal sheep serum. Primary antibodies were: anti-Sox9 (sc-166505; Santa Cruz Biotechnology), anti-p65 (sc-372; Santa Cruz Biotechnology), anti-COX-2 (610203; BD Transduction Laboratories, the Netherlands), anti-iNOS (ab3523; Abcam) and anti-Col2a1 (II-II6B3; Developmental Studies Hybridoma Bank). Similar antibody concentrations were used for negative controls; mouse IgG1 (Dako) for COX-2 and Col2a1, mouse IgG2a (Dako) for Sox9, normal rabbit serum 
for iNOS and anti-p65 blocking peptide (sc-372P; Santa Cruz Biotechnologies) for p65. After washing in PBS-T, bound antibodies were detected with HRP-labelled secondary antibodies (Dako, EnVision+ System-HRP labelled Polymer). For visualisation, DAB substrate (Dako) was used. Stained sections were counterstained with Mayer's Hematoxylin (Dako), dehydrated and mounted in Histomount as described above.

\begin{tabular}{|c|c|c|}
\hline Oligo sets mouse & Forward & $\underline{\text { Reverse }}$ \\
\hline Col2al & '5-TGGGTGTTCTATTTATTTATTGTCTTCC-3’ & '5-GCGTTGGACTCACACCAGTTAGT-3' \\
\hline Col10a1 & '5-CATGCCTGATGGCTTCATAAA-3' & '5-AAGCAGACACGGGCATACCT-3' \\
\hline Runx2 & '5-CGATGAAGACCCCAACCCTAA-3' & '5-ACTGGTAATGGCATCAAGGGATA-3' \\
\hline Sox 9 & '5-AGTACCCGCACCTGCACAAC-3' & '5-TACTTGTAGTCCGGGTGGTCTTTC-3' \\
\hline L-Sox 5 & '5-CCCAGCCACTGAACCTATCAG-3' & '5-TGTGAGGAGAGGTGGGTGATG-3' \\
\hline Sox6 & '5-AAATCCACCTAGAGAAGTACCCAAAC-3' & '5-CCCAATCCGGAGCTTCTTG-3' \\
\hline $\mathrm{COX}-2$ & '5-GGCCATGGAGTGGACTTAAA-3' & '5-AAGTGGTAACCGCTCAGGTG-3' \\
\hline iNOS & '5-CAGCTGGGGCTGTACAACCCTT-3' & '5-CATTGGAAGTGAAGCGTTTCG-3' \\
\hline Il-6 & '5-GCTACCAAACTGGATATAATCAGGAAA-3' & '5-CTTGTTATCTTTTAAGTTGTTCTTCATGTACTC-3' \\
\hline TNF $\alpha$ & '5-CATCTTCTCAAAATTCGAGTGACAA-3' & '5-TGGGAGTAGACAAGGTACAACCC-3' \\
\hline p65 & '5-ACAGACCCAGGAGTGTTCACAGA-3' & '5-CATGGAACACACCCTGGTTCAG-3' \\
\hline FGF-3 & '5-AGCGGCCGCGTGAAC-3' & '5-CCACTTCCACCGCAGTAATCTC-3' \\
\hline IGF-1 & '5-AACAAGCCCACAGGCTATGG-3' & '5-AAGCAACACTCATCCACAATGC-3' \\
\hline TGF- $\beta 3$ & '5-TCGACATGGAGCTGGTGAAA-3' & '5-GAGCCTTAGTTTGGACAGGATCTG-3' \\
\hline BMP-2 & '5-AGAAAAGCGTCAAGCGAAACA-3' & '5-GTCCACGTACAAAGGGTGTCTCT-3' \\
\hline BMP-4 & '5-ACCCGCAGCCTAGCAAGA-3' & '5-CTGACTGGAGCCGGTAAAGATC-3' \\
\hline$\beta$-Actin & '5-GACAGGATGCAGAAGGAGATTACTG-3' & '5-CCACCGATCCACACAGAGTACTT-3' \\
\hline Oligo sets human & Forward & $\underline{\text { Reverse }}$ \\
\hline COL2A1 & '5-TGGGTGTTCTATTTATTTATTGTCTTCCT-3' & '5-GCGTTGGACTCACACCAGTTAGT-3' \\
\hline COL10A1 & '5-ATGATGAATACACCAAAGGCTACCT-3' & '5-ACGCACACCTGGTCATTTTCTG-3' \\
\hline SOX9 & '5-AGTACCCGCACCTGCACAAC-3' & '5-CGCTTCTCGCTCTCGTTCAG-3' \\
\hline $\mathrm{COX}-2$ & '5-ACCAACATGATGTTTGCATTCTTT-3' & '5-GGTCCCCGCTTAAGATCTGTCT-3' \\
\hline IL-6 & '5-GCTACCAAACTGGATATAATCAGGAAA-3' & '5-CTTGTTATCTTTTAAGTTGTTCTTCATGTACTC-3' \\
\hline $28 \mathrm{~S}$ rRNA & '5-GCCATGGTAATCCTGCTCAGTAC-3' & '5-GCTCCTCAGCCAAGCACATAC-3' \\
\hline Oligo sets chicken & Forward & $\underline{\text { Reverse }}$ \\
\hline Col2a1 & ‘5-AAGAAAGCCATCCTCATCCAG-3’ & '5-CCATTTGCCAGTGTGTTTCG-3' \\
\hline Aggrecan & '5-AAGTGAAGATACCTGAGCAGTC-3' & '5-CAGCAGAGCATTGGTGTCC-3' \\
\hline Sox 9 & '5-AGTACCCGCACCTGCACAAC-3' & '5-CGCTTCTCGCTCTCGTTCAG-3' \\
\hline Cyclophillin A & '5-CCTGCTTCCACCGGATCAT-3' & '5-CGTTGTGGCGCGTAAAGTC-3' \\
\hline
\end{tabular}

Table 2.1. DNA oligo sequences for RT-qPCR.

Forward and reverse oligo sequences ( $\left.5^{\prime}-3^{\prime}\right)$ used for RT-qPCR are listed for mouse, human and chicken.

\section{$\underline{2.8 \text { Statistics }}$}

In the Figures, bars represent average value of 3 individual experiments (performed in triplicate; $3 \times 3$ samples) and error bars represent mean \pm s.e.m.. Statistical significance ( $<$ 0.05) was determined by unpaired two-tailed student t-tests using Graphpad PRISM 5.0 (La Jolla, CA, USA). 


\section{Results}

\subsection{Early ATDC5 differentiation is accompanied by a transient activation of $N F-k B / p 65$} and expression of $\operatorname{Sox} 9$

As a model for endochondral ossification, the murine chondroprogenitor ATDC5 cell line was used ${ }^{18,22}$. Early involvement of NF-KB/p65 signaling was assessed by examining subcellular localization of the NF- $\kappa \mathrm{B}$ subunit $\mathrm{p} 65^{23}$ (Figure 2.1A). In proliferating cells $(t=0)$ p65 was not detectable in the nucleus. However, upon initiation of chondrogenesis a fraction of cytoplasmically localized p65 translocated to the nucleus, which was readily detectable at 30 minutes post-induction of differentiation. The nuclear occupation of p65 peaked between one half and four hours and was not detectable anymore after eight hours (Figure S2.1). To further verify overall activation of NF- $\mathrm{BB} / \mathrm{p} 65$, expression of NF-кBtarget genes was measured (Figure 2.1B). Induction of COX-2, iNOS, Il-6 and TNF $\alpha$ mRNAs was detectable between one and four hours in differentiation and returned to baseline levels around eight hours. COX-2 and iNOS proteins showed a similar transient expression (Figure 2.1C). To verify potential cross-talk between the differentiation program and the early NF-кB/p65 response, expression of Sox9 was determined. During ATDC5 differentiation Sox9 expression was transiently induced at one to four hours in differentiation and steadily increased again from day seven in differentiation (Figure 2.1D). Remarkably, these data imply that the expression of Sox9 during chondrogenic differentiation in ATDC5 is bi-phasic. Confirming completion of the chondrogenic differentiation program in the ATDC5 cells chondrogenic markers Col2a1, collagen type X (Col10a1), and runt-related transcription factor 2 (Runx2) increased in expression from day seven in differentiation (Figure 2.1E).

Previously it was reported that an NF- $\mathrm{KB} / \mathrm{p} 65$ transcription factor binding site is located in the Sox 9 gene $^{11}$. In silico screening of Sox 9 promoter regions detected two other putative evolutionary conserved NF- $\mathrm{kB} / \mathrm{p} 65$ transcription factor binding sites in various mammals (Figure 2.2).

Post-natal growth plates contain a pool of dedicated chondroprogenitor cells in the so-called resting zone. During growth plate development, these resting zone cells differentiate into proliferating chondrocytes and are thus responsible for cartilage generation in the growth plate ${ }^{24}$. To verify whether activation of NF-KB/p65 can also be detected in early chondrogenesis during endochondral ossification in vivo, the resting- and proliferative 
zones of six week old mice growth plates were analyzed for expression of Sox9, p65 and NF-KB/p65-target genes (COX-2 and iNOS) (Figure 2.1F). As described previously ${ }^{25}$, Sox9 expression was detected in the resting zone (RZ) cells as well as in proliferative zone chondrocytes (PZ). Interestingly, Sox9 seems to be more abundantly expressed in the resting zone. Expression of p65 was found in the cytoplasm of RZ cells and was not detectable in the PZ. Also, p65 was found to be localized in the nuclei of several RZ cells. Both the expression of the NF-KB/p65-target genes COX-2 and iNOS was found in the RZ. Overall, these results indicate that an NF-KB/p65 signaling response occurs very early in chondrogenic differentiation and correlates with a thusfar unknown early transient induction of Sox9 in ATDC5 cells.

$\mathrm{C}$
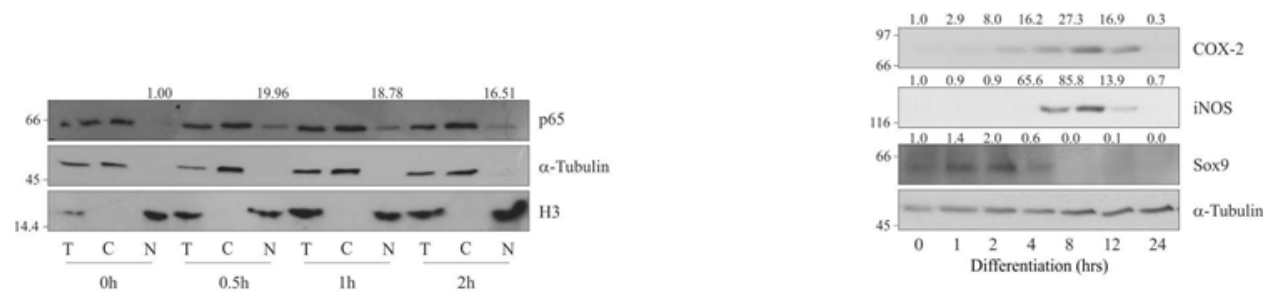

B

$\operatorname{cox}-2$

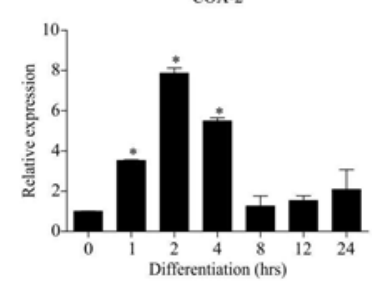

II-6

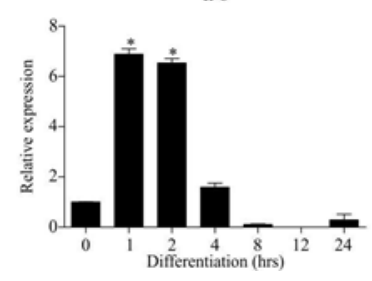

iNOS

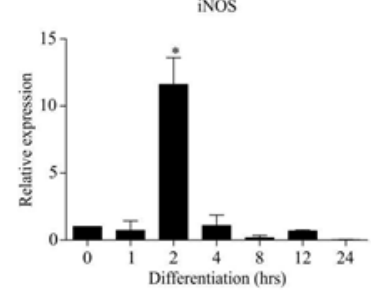

TNF $\alpha$

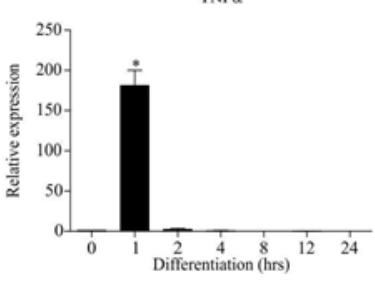

D

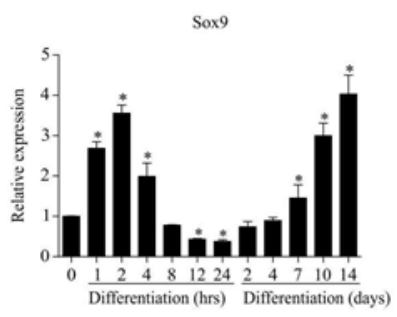


E
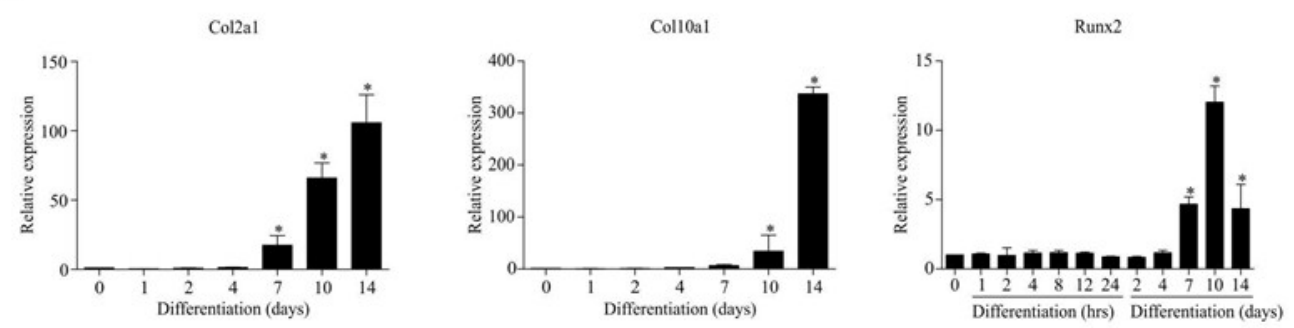

F

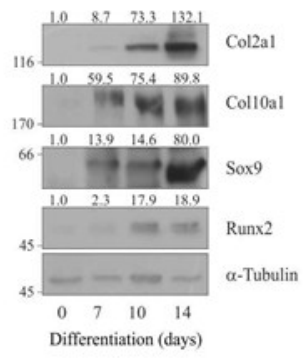

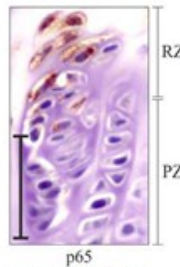

p65

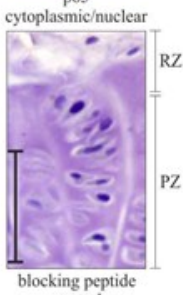

blocking peptide

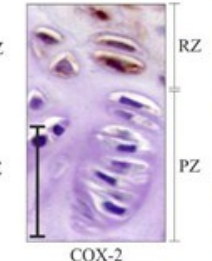

$\mathrm{COX}-2$

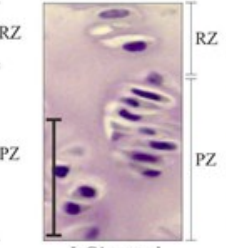

IgGl control

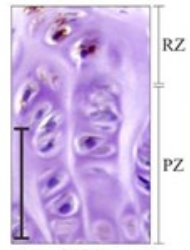

iNOS

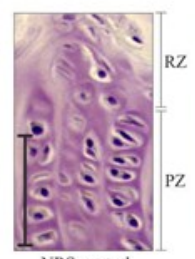

NRS control

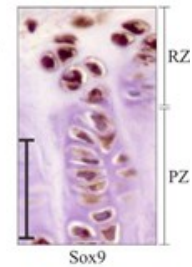

Sox 9

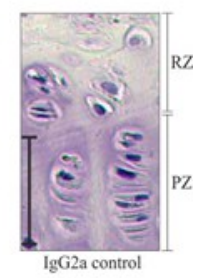

Figure 2.1: Early chondrogenic differentiation is accompanied by $N F-\kappa B / p 65$ activation and transient Sox 9 expression

(A). Nuclear translocation of the NF- $\mathrm{kB}$ subunit $\mathrm{p} 65$. Total extract $(\mathrm{T})$, cytoplasmic $(\mathrm{C})$ and nuclear $(\mathrm{N})$ fractions were isolated at 0, 0.5, 1 and 2 hours in ATDC5 differentiation. Cytoplasmic marker: $\alpha$-Tubulin, nuclear marker: Histone H3. (B). COX-2, iNOS, Il-6 and TNF $\alpha$ mRNA expression at 0-24 hours in differentiation (relative to $\mathrm{t}=0$ and corrected for $\beta$-actin). ${ }^{*}=\mathrm{p}<0.05$. (C). Protein expression of COX-2, iNOS and Sox9 at 0-24 hours in differentiation. Molecular weight markers $(\mathrm{kDa})$ are depicted on the left of immunoblots and relative quantifications are depicted on top of immunoblots. (D). Sox9 mRNA expression during chondrogenic differentiation. $(E)$. mRNA and protein expression of Col2a1, Col10a1, Runx2 and Sox9 (only protein) at day 0, 7, 10 and 14 in ATDC5 differentiation. $(F)$. Sections from 6 weeks old mouse growth plates (resting (RZ) and proliferative (PZ) zones) stained for p65, COX-2, iNOS and Sox9. Lower panels show appropriate negative controls. Bars $=50 \mu \mathrm{m}$. 


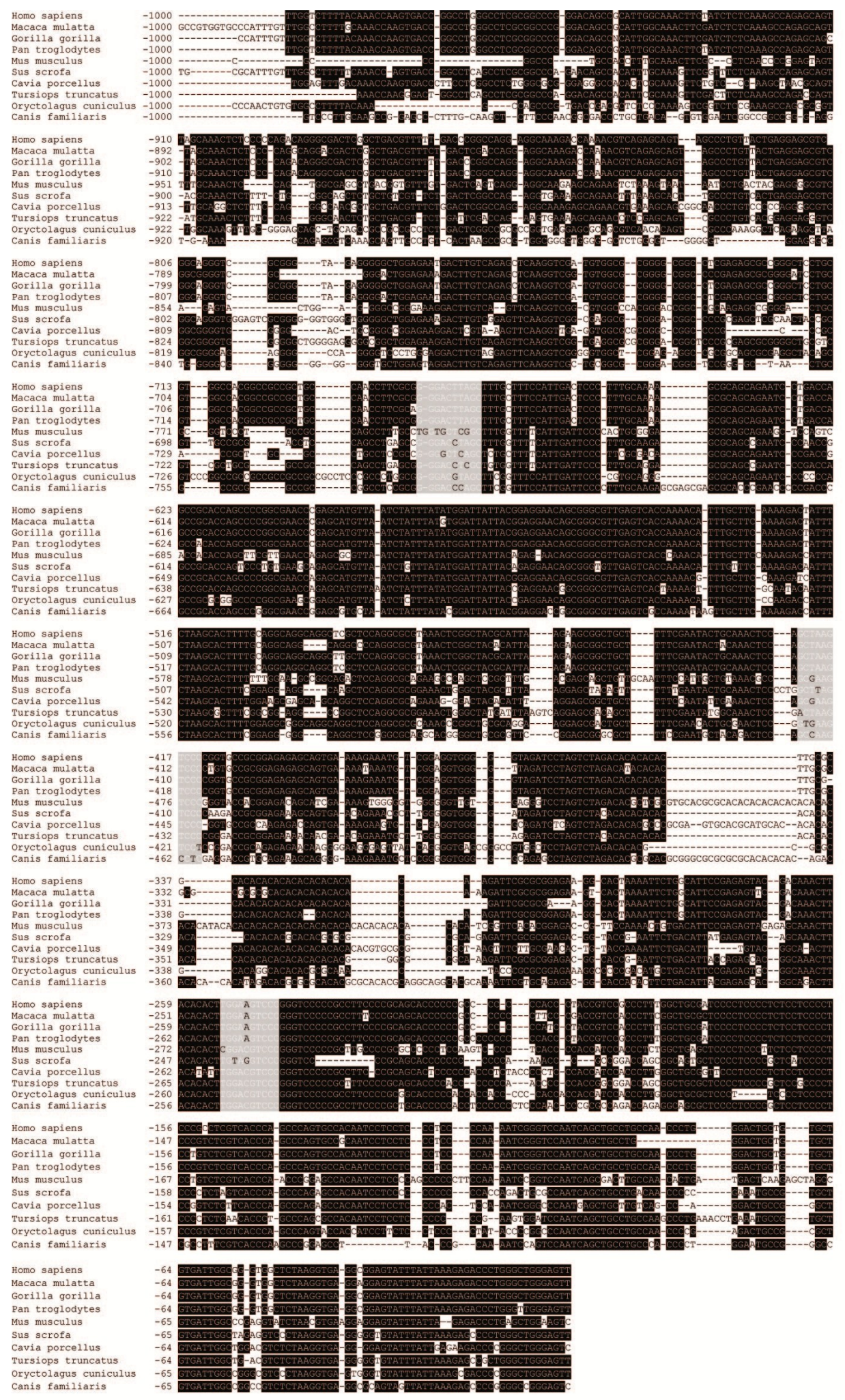


Figure 2.2: In silico scan for $N F-\kappa B / p 65$ transcription factor binding sites in the Sox 9 promoter.

Genomatix's Gene2Promoter software (www.genomatix.de) was used to screen Sox9-promoters (-1000 bp region) from ten different vertebrates for putative NF- $\mathrm{BB}$ transcription factor binding sites, based on the consensus binding sequence for NF- $\mathrm{BB}$ : GGGRNNYYCC (in which $\mathrm{R}=$ purine, $\mathrm{N}=$ any nucleotide and $\mathrm{Y}=$ pyrimidine $)^{26}$. A multiple nucleotide sequence alignment was generated by the T-Coffee multiple sequence alignment program (http://www.tcoffee.org/) and $>50 \%$ nucleotide homology is depicted in black boxes generated by the Boxshade server v3.21 (http://www.ch.embnet.org/software/BOX_form.html). Evolutionary conserved putative $\mathrm{NF}-\kappa \mathrm{B}$ transcription factor binding sites are marked in grey boxes. In addition to the functional NF- $\mathrm{B}$ transcription factor binding sites found by Ushita and colleagues ${ }^{27}$, two other putative evolutionary conserved NF- $\mathrm{KB}$ transcription factor binding sites were detected. Ensembl references to used promoter sequences are Homo sapiens: GRCh37:17:70116161:70117161:1, Macaca mulatta: MMUL_1:16:67356426:67357426:1, Gorilla gorilla: gorGor3:5:1129374:11298374:-1, Pan troglodytes: CHIMP2.1:17:71547493:71548493:1, Mus musculus: NCBIM37:11:112642524:112643524:1, Sus scrofa: Sscrofa9:12:6872241:6871241:-1, Cavia porcellus: cavPor3:scaffold_3:74364652:74365652:1, Tursiops truncates: turTru1:scaffold_92737:81033:82033:1, Oryctolagus caniculus: oryCun2:19:55819664:55820664:1, Canis familiaris: BROADD2:9:11347542:11348542:1

\subsection{Inhibition of early $N F-\kappa B / p 65$ activation leads to impaired chondrogenic differentia-}

$\underline{\text { tion }}$

To functionally determine the role of the early NF-KB/p65 activation in relation to initiation of chondrogenic differentiation, nuclear translocation of NF- $\mathrm{KB}$ was inhibited by TLCK or Parthenolide. Dose-response experiments (data not shown) revealed optimal inhibitor concentrations: $100 \mu \mathrm{M}$ TLCK or $10 \mu \mathrm{M}$ Parthenolide efficiently inhibited NF- $\mathrm{BB}$ nuclear translocation during early ATDC5 chondrogenesis (Figure 2.3A). In agreement with efficient NF- $\kappa$ B inhibition, both TLCK and Parthenolide inhibited COX-2, iNOS and Il-6 mRNA expression at two hours in differentiation (Figure 2.3B).

We next tested the functional relationship between the early $\mathrm{NF}-\mathrm{KB} / \mathrm{p} 65$ response and chondrogenic differentiation. Early Sox9 levels were reduced by both TLCK and Parthenolide (Figure 2.3B and Figure 2.5A). Inhibition of NF- $\kappa \mathrm{B}$ activation by TLCK or Parthenolide resulted in a similar dose-dependent inhibition of late phase (day 14) Col2a1, Col10a1, Runx2 and Sox9 protein expression (Figure 2.3C). Results were confirmed using the clinically used NF- $\mathrm{BB}$ inhibitor Sulfasalazine (Figure 2.3C).

To independently verify the effect of pharmacological NF-kB/p65 inhibition on Sox 9 expression during early chondrogenic differentiation, we genetically targeted NF- $\mathrm{kB} / \mathrm{p} 65$ by RNAi. Transient transfection of a p65 siRNA duplex in ATDC5 reduced p65 mRNA and protein expression at two hours in differentiation by $\sim 50 \%$ (Figure $2.3 \mathrm{D}$, upper left 
set). Expression of COX-2, iNOS and Il-6 were also reduced as compared to control transfection (data not shown). In good agreement with the results described above, early expression of Sox9 mRNA and protein was significantly reduced by p65 knock-down (Figure 2.3D, upper right set). To further validate how p65 knock-down affects long term chondrogenic differentiation, p65 mRNA expression was targeted for a longer timeframe by re-transfection of the siRNA duplex. We confirmed efficient knock-down of p65 mRNA at ten days in differentiation (Figure 2.3D, upper left and lower left sets). Functionally the p65 knock-down resulted in impaired chondrogenic differentiation (Figure 2.3D, lower left and lower right). Finally, to test whether the early transient Sox 9 induction influences late chondrogenic differentiation we targeted the expression of Sox 9 only in the early phase of differentiation by one single Sox9 siRNA transfection, followed by seven days of differentiation follow-up under normal differentiation conditions. Efficient knock-down of Sox9 mRNA was confirmed at the start of differentiation. At two hours in differentiation the early induction of Sox 9 was almost completely abolished (Figure 2.3E, upper panel). At day four, Sox9 siRNA treatment was not effective anymore, as no difference in Sox9 expression was detectable between conditions. To verify the consequence of this early Sox9 knock-down, the expression of Col2a1 was determined (Figure 2.3E, lower panel). Col2a1 expression was detectable from day four onwards. At day four a significant difference was found between control and the early Sox9 knockdown. This difference was even bigger at day seven, where early Sox9 knock-down lead to an almost abolished expression of Col2a1.

Taken together, these results indicate that inhibition of $\mathrm{NF}-\kappa \mathrm{B} / \mathrm{p} 65$ nuclear translocation suppresses the initiation of chondrogenic differentiation, by inhibiting early Sox9 induction and subsequent expression of late chondrogenic markers. This indicates that late phase chondrogenic development and endochondral ossification are, at least in part, regulated by early NF-kB/p65 and Sox 9 signaling events. 
A

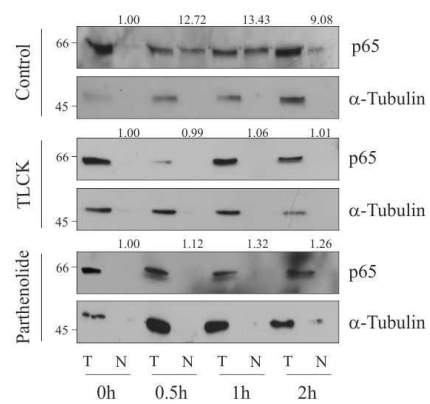

B
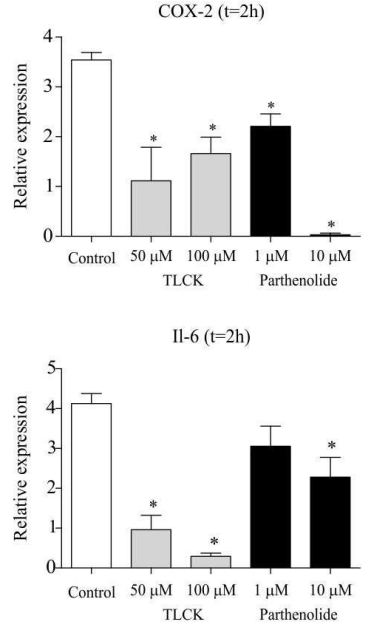

D
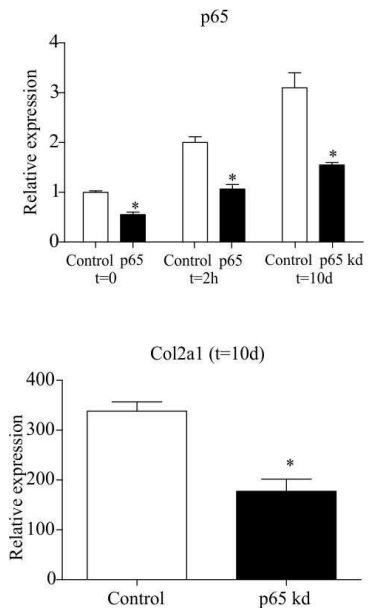

C
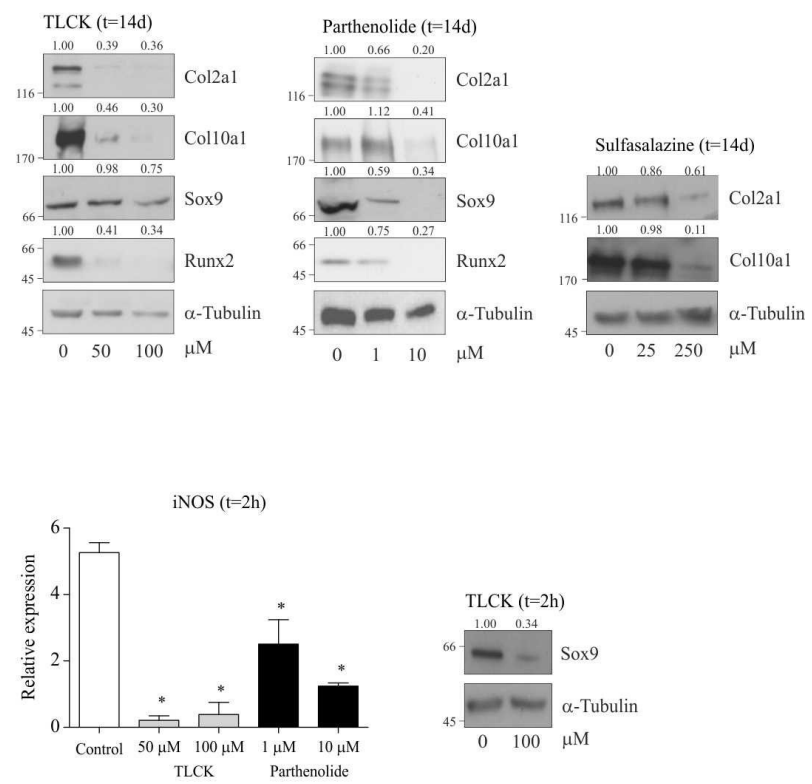

Sox9 $(\mathrm{t}=2 \mathrm{~h})$
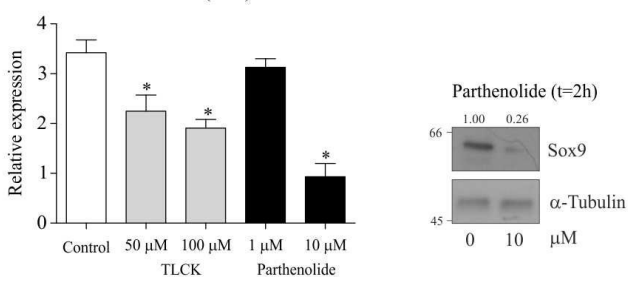
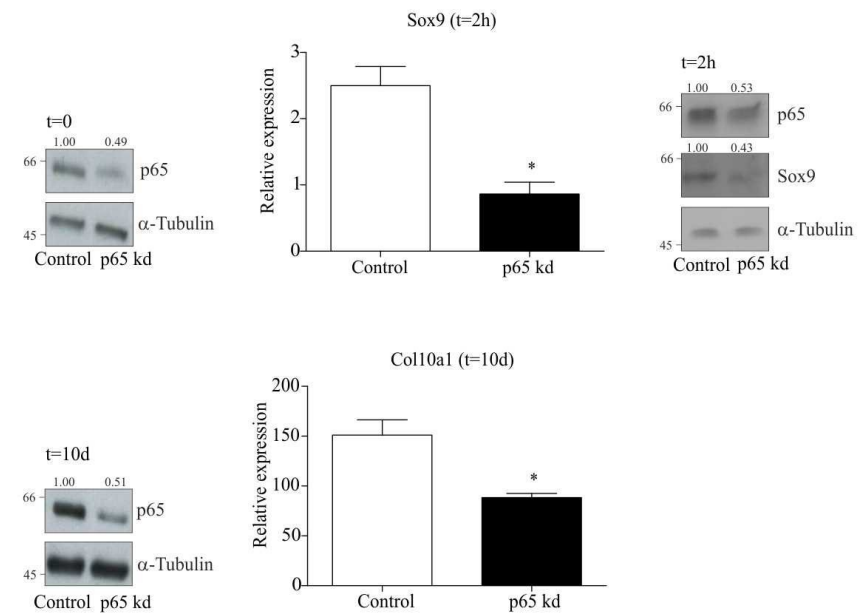
$\mathrm{E}$
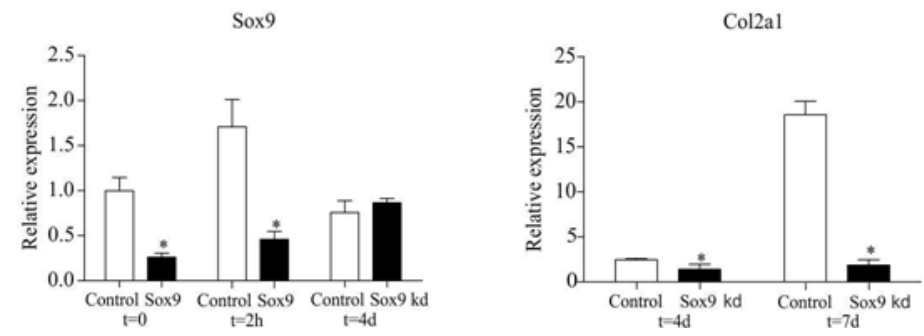

Figure 2.3: Inhibition of early $N F-\kappa B / p 65$-activation leads to impaired ATDC5 differentiation

(A). Cells were differentiated in the absence (control) or presence of TLCK $(100 \mu \mathrm{M})$ or Parthenolide $(10 \mu \mathrm{M})$ and total $(\mathrm{T})$ and nuclear $(\mathrm{N})$ fractions were prepared. NF- $\mathrm{\kappa B}$ was detected as p65. (B). Expression of COX-2, iNOS, Il-6 and Sox 9 mRNAs at 2 hours in differentiation in the presence of TLCK or Parthenolide. $*=\mathrm{p}<0.05$. Sox9 protein expression was determined from similar samples (right panel set). (C). Protein expression at day 14 in differentiation of Col2a1, Col10a1, Sox9 and Runx2 with TLCK (left panels), Parthenolide (right panels) or Sulfasalazine (lower left panels). (D). Knock-down (kd) of p65 mRNA and protein at 0, 2 hours and 10 days in differentiation ("Control" below figures indicates scrambled siRNA and "p65" below figures indicates p65 kd). Upper right set: expression of Sox 9 mRNA and protein in p65 kd cells at 2 hours in differentiation. Lower sets: messenger RNA expression of Col2al and Col10a1 at 10 days in differentiation of p65 kd cells. (E). Sox 9 mRNA expression at 0,2 hours and 4 days in differentiation of cells transfected one day prior to differentiation with scrambled (indicated by "Control") or Sox9 siRNA (indicated by "Sox9") (upper panel). Col2a1 mRNA expression was determined at day 4 and 7 in differentiation (lower panel).

\subsection{Late phase ATDC5 differentiation is enhanced by stimulation of early $N F-\kappa B / p 65$ ac-} tivity

The association between early NF- $\mathrm{BB} / \mathrm{p} 65$ activation and initiation of chondrogenic differentiation prompted us to test whether stimulation of NF-KB/p65 enhances chondrogenic differentiation. We enforced NF-kB/p65 signaling by supplementing differentiation medium with the NF- $\kappa \mathrm{B} / \mathrm{p} 65$-activating molecular tools LPS or TNF $\alpha$. These activating agents were added to the differentiating culture during the first 24 hours of differentiation only (the timeframe in which the early NF-KB/p65 activation takes place). At low LPS concentrations $(0.1 \mathrm{ng} / \mathrm{ml})$ and $\mathrm{TNF} \alpha(10 \mathrm{ng} / \mathrm{ml}), \mathrm{NF}-\kappa \mathrm{B} / \mathrm{p} 65$ nuclear translocation was slightly enhanced during early chondrogenic differentiation (Figure 2.4A) and resulted in increased early expression of COX-2, Il-6 and iNOS mRNAs (Figure 2.4B). Interestingly, increasing NF- $\mathrm{KB} / \mathrm{p} 65$ activity enhanced the magnitude of transient Sox9 (as well as L-Sox5 and Sox6) expression early in chondrogenic differentiation (Figure 2.4B and Figure 2.5A). Interference with NF-KB-signaling by siRNA mediated p65 knock-down attenuated the LPS- and TNF $\alpha$-induced increased Sox9 expression back to control-treated 
differentiation levels (Figure 2.4C). Moreover, increased Col2a1, Col10a1, Sox9 and Runx2 expression in 24 hour LPS- and TNF $\alpha$-exposed cells was detected at day ten and 14 in differentiation (Figure 2.4D and Figure 2.5B). These data show that early and short chondrogenic $\mathrm{NF}-\mathrm{kB} / \mathrm{p} 65$-activation positively responds to environmental stimulation of $\mathrm{NF}-\kappa \mathrm{B} / \mathrm{p} 65$, resulting in overall increased chondrogenic potential late in differentiation.

A

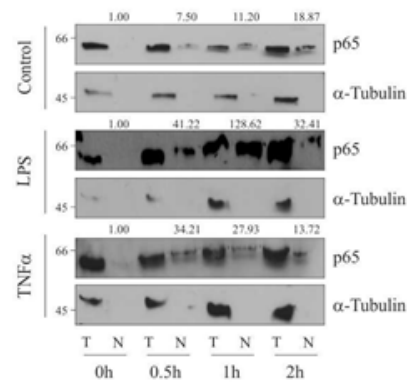

B
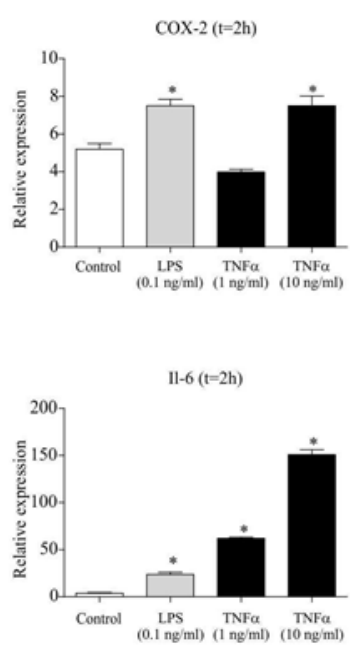

$\mathrm{C}$
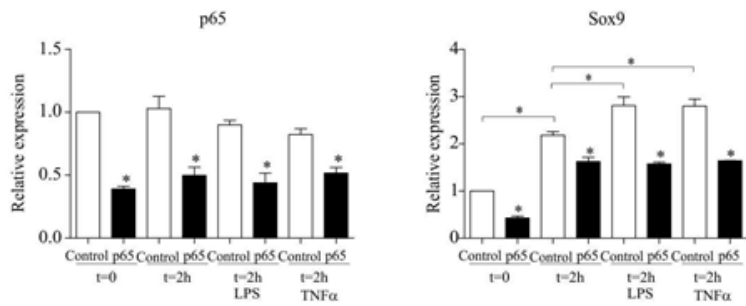

D

iNOS ( $(\mathrm{t}=2 \mathrm{~h})$
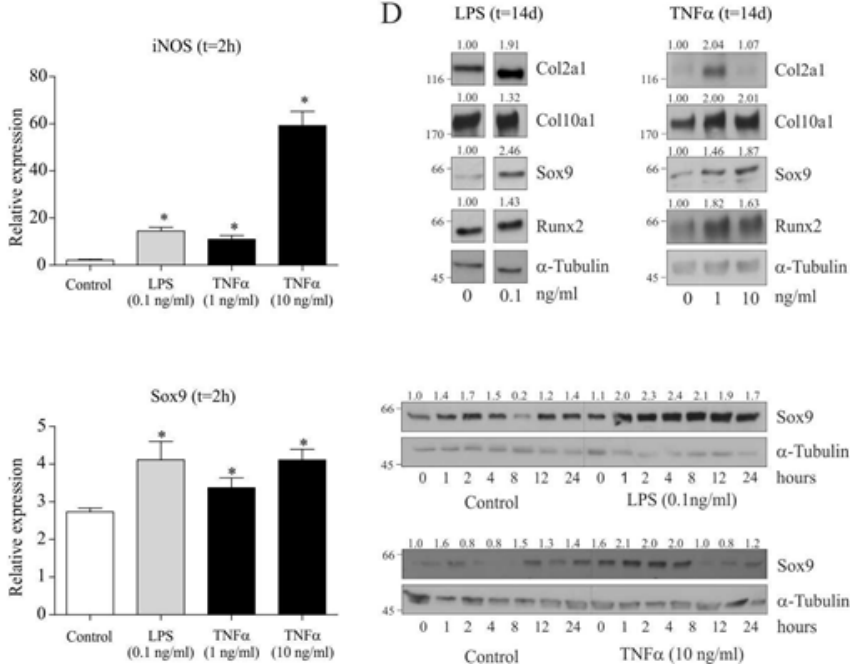

Figure 2.4: Late phase ATDC5 differentiation is enhanced by stimulation of early $\mathrm{NF- \kappa B/p} 65$ activity

(A). Cells were differentiated in the absence (control) or presence of LPS $(0.1 \mathrm{ng} / \mathrm{ml})$ or TNF $\alpha(10 \mathrm{ng} / \mathrm{ml})$ and total $(\mathrm{T})$ and nuclear $(\mathrm{N})$ fractions were prepared. NF- $\mathrm{kB}$ was detected as p65. (B). Expression of COX-2, iNOS, Il- 6 and Sox 9 mRNAs at 2 hours in differentiation in LPS or TNF $\alpha$ treated cells. Far right panel set: early Sox 9 protein expression in LPS or TNF $\alpha$ treated cells. $(C)$. ATDC5 cells were transfected with a p65 siRNA duplex or scrambled ("Control") siRNA duplex and differentiated in the absence or presence of LPS ( $0.1 \mathrm{ng} / \mathrm{ml})$ or TNF $\alpha$ $(10 \mathrm{ng} / \mathrm{ml})$ for 2 hours. Knock-down of p65 mRNA was confirmed at 0 and 2 hours in differentiation (left panel). Right panel shows Sox9 mRNA expression. (D). Protein expression of Col2a1, Col10a1, Sox9 and Runx2 in differentiated ATDC5 cells (14 days) in the presence of LPS (left panel set) or TNF $\alpha$ (right panel set), only during the first 24 hours of differentiation. $*=p<0.05$. 
A
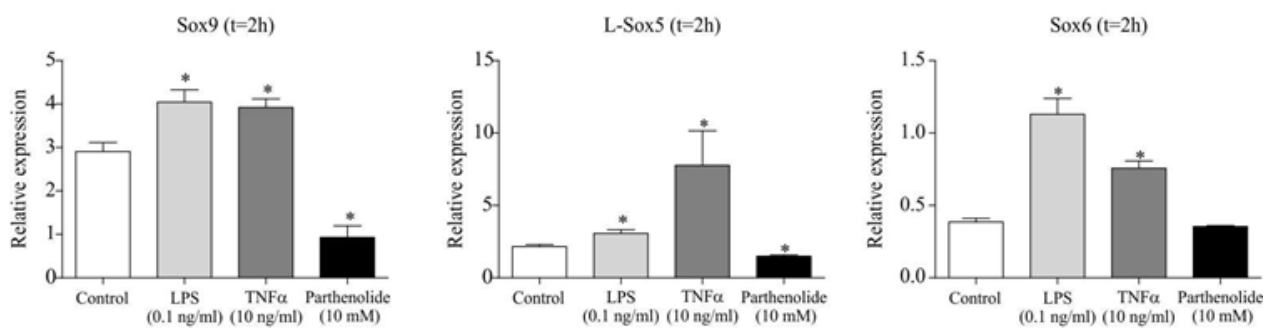

B
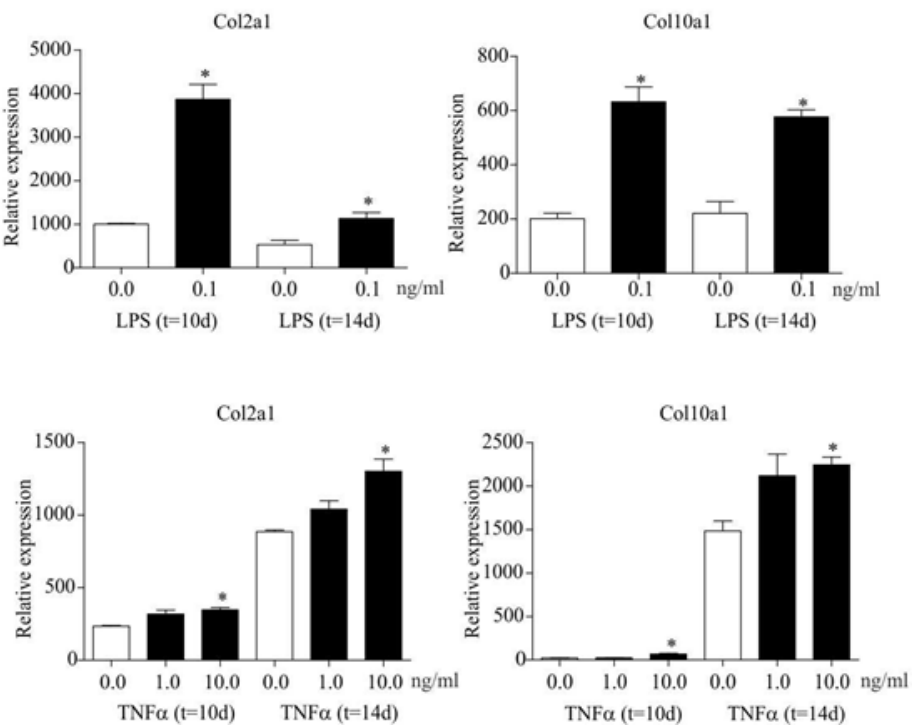

Figure 2.5: Chondrogenic differentiation is enhanced by stimulation of $\mathrm{NF}-\kappa \mathrm{B} / \mathrm{p} 65$ activity.

(A). ATDC5 was cultured for 2 hours in the absence or presence of LPS $(0.1 \mathrm{ng} / \mathrm{ml}), \mathrm{TNF} \alpha(10 \mathrm{ng} / \mathrm{ml})$ or Parthenolide $(10 \mu \mathrm{M})$ and mRNA expression of the "Sox-trio"; Sox9, L-Sox5 and Sox6 was determined. Although Sox6 expression was generally low, similar responsivity to NF- $\mathrm{kB}$ activation or inhibition were obtained for LSox 5 and Sox6 expression, supporting the suggestion that Sox 9 might function in early chondrogenic differentiation in conjunction with L-Sox5 and Sox6. (B). Col2a1 and Col10a1 gene expression data from Figure 2.4D. * $=$ $\mathrm{p}<0.05$.

\subsection{Early transient $N F-\kappa B / p 65$ signaling during endochondral differentiation of primary} mesenchymal progenitor cells

To verify the results obtained with the ATDC5 system, similar endochondral differentiation experiments were performed using human bone marrow stem cells (hBMSC). In hBMSCs, COL2A1 and COL10A1 mRNA and protein expression was evident from day 721 in chondrogenic differentiation (Figure 2.6D). Expression of COL1A1 and PPAR $\gamma$ was 
not upregulated, ruling out osteogenic or adipogenic differentiation from this multipotent cell source during chondrogenic induction (data not shown).

In concordance with ATDC5, p65 translocated to the nucleus at one hour in hBMSC differentiation and nuclear p65 levels further sustained up to four hours (Figure 2.6A). Analyses showed that hBMSC chondrogenic differentiation of three independent isolates is accompanied by transient expression of COX-2 and IL-6 early in differentiation (Figure 2.6B). Furthermore, as in ATDC5, induction of SOX9 expression in hBMSC differentiation was also bi-phasic: from one and two hours onward, as well as late (day 1428 ) in differentiation (Figure 2.6C). In agreement with reduced NF- $\kappa \mathrm{B} / \mathrm{p} 65$ activity, COX2, SOX9 and IL-6 expression levels decreased in the presence of TLCK (Figure 2.6E; grey

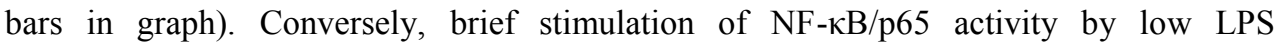
concentrations during the first 24 hours only, enhanced NF- $\mathrm{kB} / \mathrm{p} 65$-target gene expression, as well as early SOX9 expression (Figure 2.6E; black bars in graph). Coherent with the murine model, human chondroprogenitor cells clearly showed increased expression of COL2A1 at day 21 when exposed to LPS during the first 24 hours in differentiation (Figure 2.6F; lanes 2) and lower COL2A1 levels in the presence of TLCK (Figure 2.6F; lanes 3). Taken together, these data show that hBMSC endochondral differentiation also integrates a transient NF- $\mathrm{KB} / \mathrm{p} 65$ activation during the early initiation of differentiation, ultimately contributing to the outcome of the chondrogenic cell fate.

\section{$3.5 \mathrm{NF- \kappa B} / \mathrm{p} 65$ signaling induces chondrogenic marker gene expression in mesenchymal}

\section{progenitor cells without the addition of chondrogenic growth factors}

The progenitor cell intrinsic NF- $\mathrm{kB} / \mathrm{p} 65$ activation occurs as a result of environmental

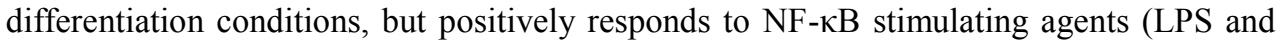
$\mathrm{TNF} \alpha$ ) early in chondrogenic differentiation. We therefore tested whether an NF- $\kappa \mathrm{B} / \mathrm{p} 65$ activating stimulus alone would be able to facilitate chondrogenic signaling in mesenchymal progenitors, without the addition of other differentiation factors like insulin or TGF- $\beta 3$. To this end, ATDC5 proliferation medium was supplemented with only LPS (first 24 hours alone) and subsequently cells were cultured in proliferation medium for ten

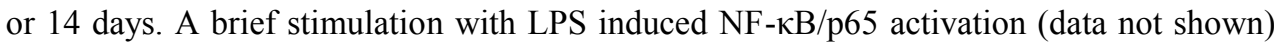
and, in contrast to the control condition, resulted in a transient Sox9 expression at two hours after LPS exposure (Figure 2.7A, lower left panel) and equal Sox9, Col2a1 and Col10a1 mRNA expression levels (at day ten and 14) as normal differentiation conditions do in ATDC5 (Figure 2.7A). However Col10a1 expression in the proliferation condition at 
$\mathrm{A}^{-}$

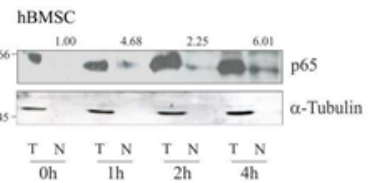

$\mathrm{C}$

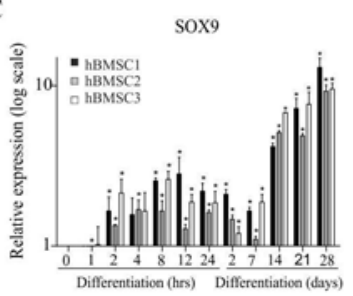

D

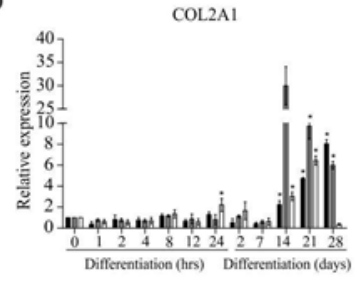

E

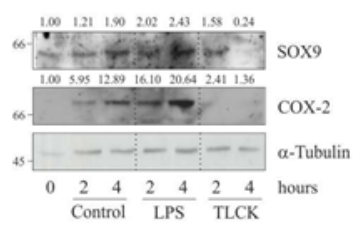

B
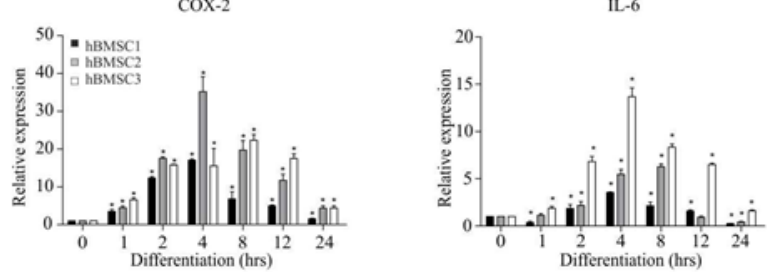

F
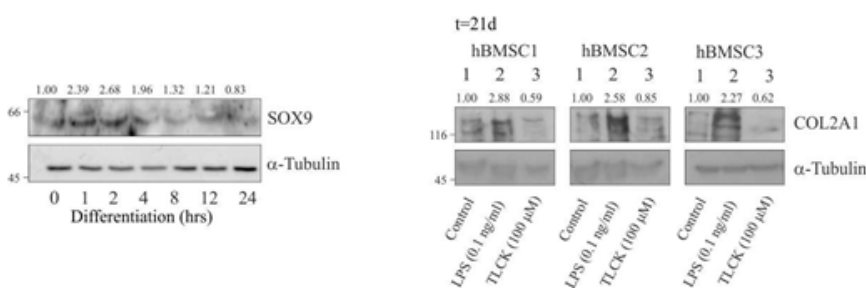

COL10A1
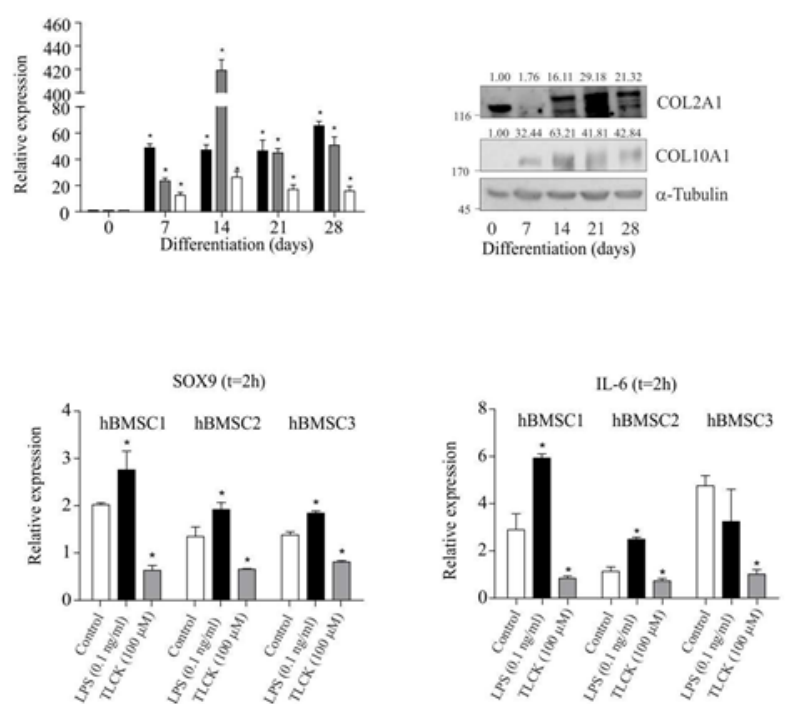

Figure 2.6: Transient $N F-\kappa B / p 65$ signaling during early chondrogenic differentiation of human bone marrow stem cells

Human bone marrow stem cells from three individuals (hBMSC1/2/3) were differentiated into the chondrocyte lineage using monolayer culture. $(A)$. Nuclear $(\mathrm{N})$ and total $(\mathrm{T})$ fractions were isolated from 0, 1, 2, 4 hours samples. NF- $\mathrm{BB}$ was detected as p65, cytoplasmic marker: $\alpha$-tubulin. (B). Expression of COX-2 and IL-6 mRNAs at 0 -24 hours in hBMSC differentiation. $(C)$. Left; SOX9 mRNA expression during hBMSC differentiation, SOX9 protein expression during 0-24 hours in hBMSC differentiation (right) (D). COL2A1 and COL10A1 mRNA and protein expression during hBMSC differentiation. (E). Left; SOX9 and COX-2 protein expression at 0, 2, 4 hours in differentiation in the presence of LPS $(0.1 \mathrm{ng} / \mathrm{ml})$ or TLCK $(100 \mu \mathrm{M})$. Right; SOX9 and IL-6 mRNA expression at 4 hours in differentiation in the presence of LPS (black bars) or TLCK (grey bars). $(F)$. COL2A1 protein expression in day 21-samples of differentiated hBMSCs. Lanes 1: control condition, Lanes 2: $0.1 \mathrm{ng} / \mathrm{ml}$ LPS (only first 24 hours) and lanes 3: $100 \mu \mathrm{M}$ TLCK. $*=p<0.05$. 
day 14 appeared unexpectedly high as compared to the LPS and differentiation conditions. Briefly, findings were verified in hBMSCs. After 21 days culture in proliferation medium (without insulin and TGF- $\beta 3$ ), hBMSCs expressed SOX9 very lowly and COL2A1 and COL10A1 were not expressed (Figure 2.8B), whereas brief stimulation with LPS (0.1 and $0.01 \mathrm{ng} / \mathrm{ml}$ ) during the first 24 hours only, resulted in robust expression of SOX9, COL2A1 and COL10A1 protein at 21 days (Figure 2.7B).

To further establish the relevance of our findings in tissue involved in endochondral ossification, we adopted an ex vivo periosteal tissue differentiation model for chondrogenesis using chicken embryonal periosteum ${ }^{20}$. Importantly, as a source for mesenchymal progenitor cells, periosteal tissue is directly relevant for endochondral ossification processes and fracture healing. Harvested periosteal tissue from the chicken tibia was cultured between agarose layers (chicken periosteum agarose culture: cPAC). After one week of culturing in proliferation medium chicken periosteal explants did not acquire any chondrogenic properties (Figure 2.7C; black control bars and Figure 2.7D; left micrographs). In contrast, supplementation of the culture medium with LPS for the first 48 hours only, resulted in the formation of cartilaginous tissue after one week, as determined by upregulation of Col2a1, Col10a1, Sox9 and aggrecan mRNA expression (Figure 2.7C; second black bars), as well as positive Safranin O staining, immunohistochemical detection of Sox9 and Col2a1 as well as typical chondrocyte morphology (Figure 2.7D). For comparative purposes, same chondrogenic markers were measured in cPACs that were differentiated in standard differentiation medium (containing insulin and TGF- $\beta 3$ ) (Figure 2.7C; grey bars). These data indicate that in mesenchymal progenitor cells a short exogenous NF- $\mathrm{kB} / \mathrm{p} 65$-activating stimulus may result in cellular signaling through chondrogenic pathways which can explain the expression of chondrocyte marker molecules.

\subsection{BMP-2 activates $N F-\kappa B / p 65$ in early ATDC5 chondrogenic differentiation}

LPS and TNF $\alpha$ were used as tools to activate NF-kB/p65. Exceptions left alone (e.g. TNF $\alpha$ in osteoarthritis (OA) and rheumatoid arthritis (RA)), these activators are not known to be present in the cartilaginous environment. We therefore asked whether growth factors may support the initiation of an early NF- $\kappa \mathrm{B} / \mathrm{p} 65$ activation in the way described herein. BMP's are known to play crucial roles in early mesenchymal condensation by regulating Sox 9 expression $^{28}$ and contributing to other phases of the endochondral ossification processes. Also, BMP-2 has been described to be able to activate NF- $\mathrm{KB} / \mathrm{p} 65$ in chondrocytes ${ }^{29}$. As shown in Figure 2.8A, $30 \mathrm{ng} / \mathrm{ml}$ BMP-2 resulted in increased expression of Col2a1 and 
Chapter 2

A

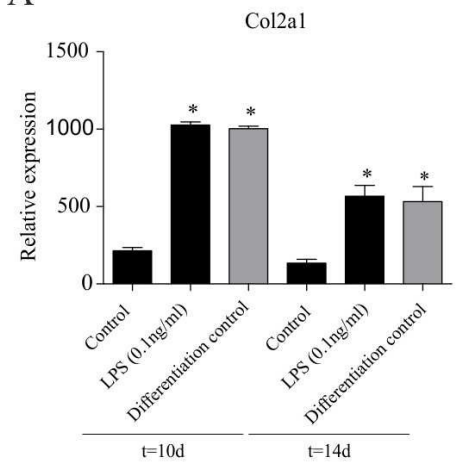

$\operatorname{Sox} 9(t=2 h)$

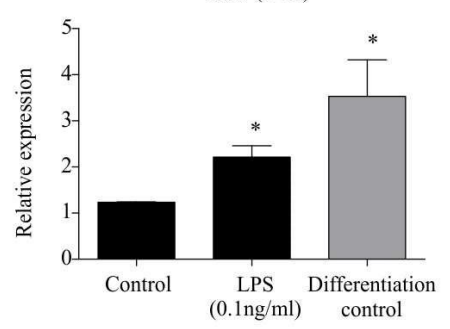

C
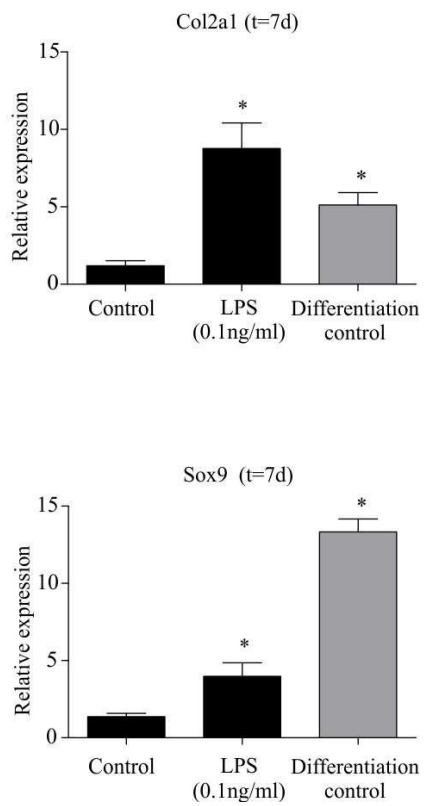

Colloal

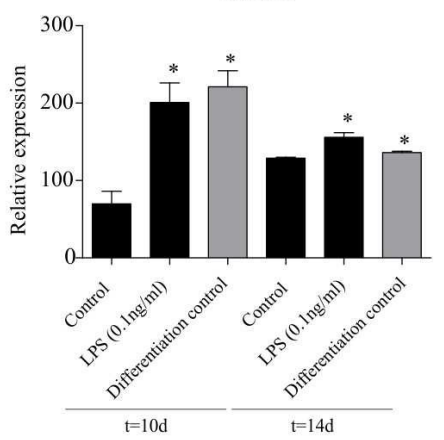

Sox 9

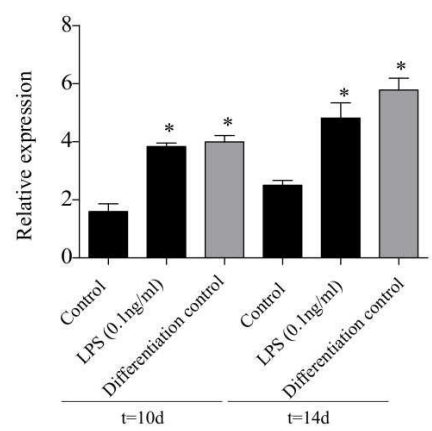

Col10al $(t=7 d)$

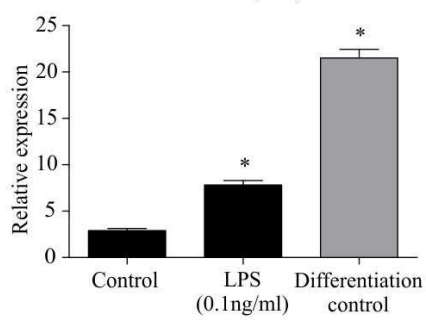

Aggrecan $(t=7 d)$

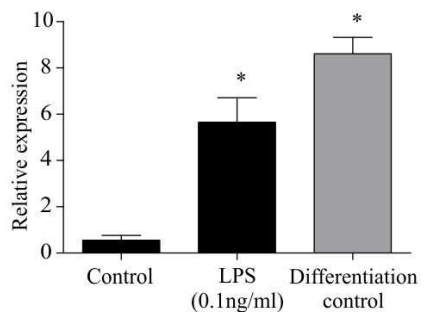

B

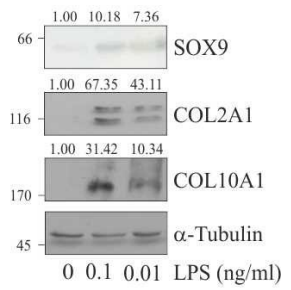


D

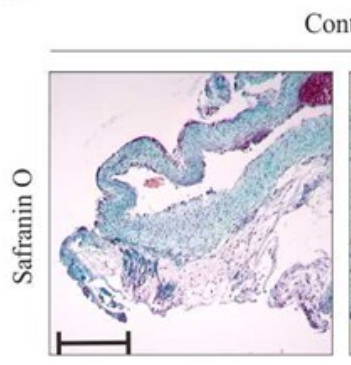

Control

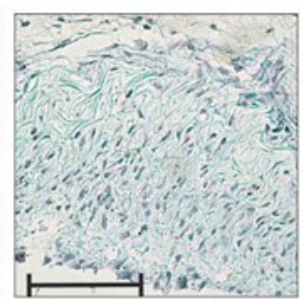

Control
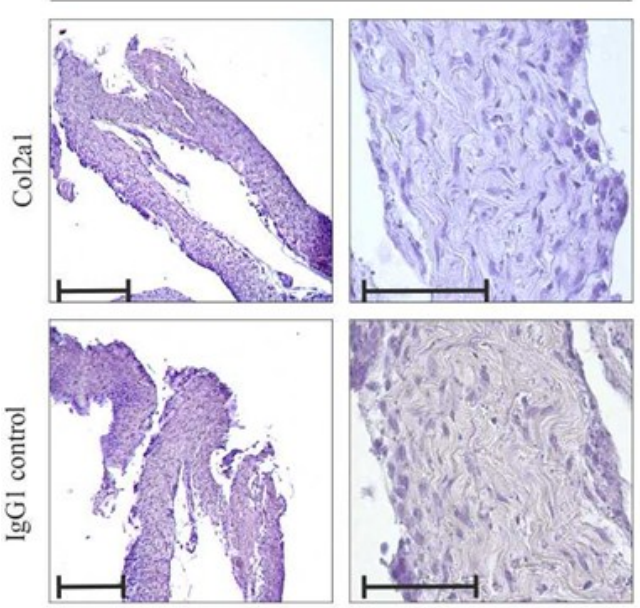

Control
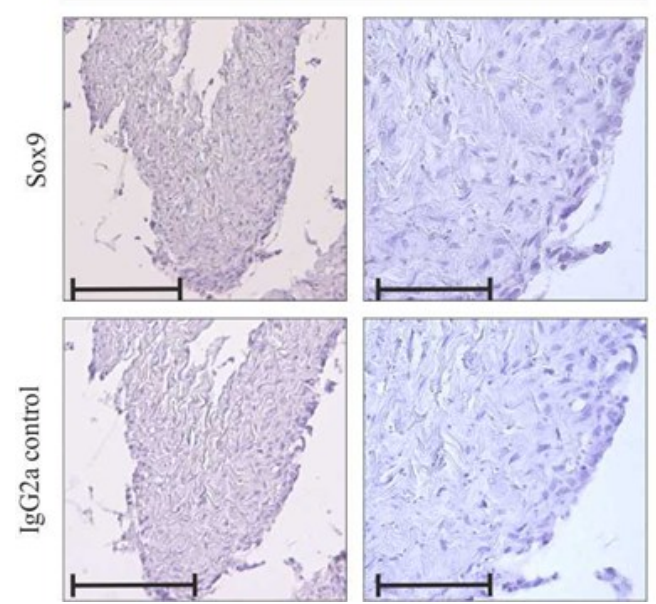

LPS (1 ng/ml)
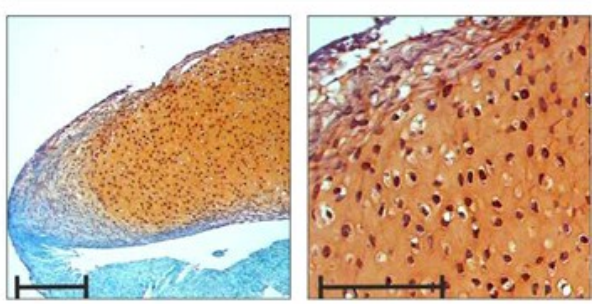

LPS $(1 \mathrm{ng} / \mathrm{ml})$
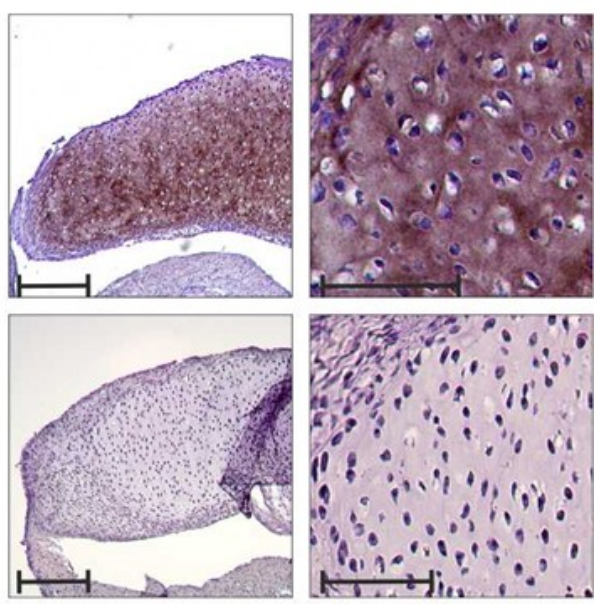

LPS ( $1 \mathrm{ng} / \mathrm{ml})$
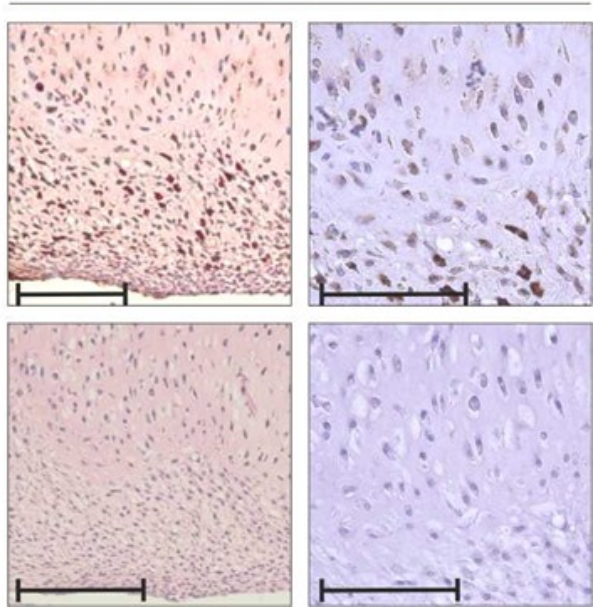


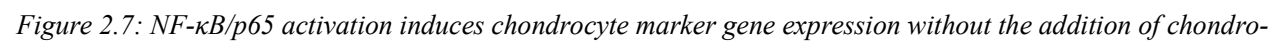
genic growth factors

(A). Col2a1, Col10a1 and Sox9 mRNA expression at 2 hours (Sox9 only), 10 and 14 days in ATDC5 cells, cultured in proliferation medium in the absence ("control”) or presence (first 24 hours) of LPS (black bars). Col2a1, Col10a1 and Sox9 mRNA expression of standard differentiated ATDC5 is shown for comparative purposes (grey bars; "differentiation control"). (B). SOX9, COL2A1 and COL10A1 protein expression in a representative hBMSC sample cultured for 21 days in proliferation medium with 0.1 or $0.01 \mathrm{ng} / \mathrm{ml} \mathrm{LPS} \mathrm{(first} 24$ hours). (C). Col2a1, Col10a1, Sox9 and aggrecan mRNA expression in cPACs (chicken Periosteum Agarose Culture) cultured in proliferation medium for 7 days in the absence or presence of LPS during the first 48 hours (black bars). Col2a1, Col10a1, Sox9 and aggrecan mRNA expression of cPACs differentiated in standard differentiation medium (containing TGF- $\beta 3$ and insulin, see also Materials and Methods) is shown for comparative purposes (grey bars). $*=\mathrm{p}<0.05$. (D). In similar samples from $(C)$ sections $(5 \mu \mathrm{m})$ from cPACs were stained by Safranin $\mathrm{O} /$ Fast green (upper set), for Col2a1 (middle set) and Sox9 (lower set). For Safranin O and Col2a1 stainings: bars = $200 \mu \mathrm{m}$ for first and third column micrographs and $100 \mu \mathrm{m}$ for second and fourth column of micrographs. For Sox9 staining, bars $=150 \mu \mathrm{m}$ for first and third column of micrographs and $100 \mu \mathrm{m}$ for second and fourth column of micrographs.

Col10a1 in differentiating ATDC5 cells. To verify whether a similar early NF-kB/p65 activation might involve this BMP-2 action, we analyzed p65 nuclear translocation. We found that p65 nuclear translocation at two hours in differentiation was more increased in the presence of BMP-2 as compared to control (Figure 2.8B). Increased and more prolonged expression of Sox 9, COX-2 and iNOS in the first 24 hours of differentiation confirmed downstream NF- $\mathrm{kB} / \mathrm{p} 65$-activated pathways (Figure 2.8C).

To further establish a role for p65 in this process, ATDC5 cells were transfected with a p65 siRNA duplex or scrambled siRNA duplex and differentiated in the absence or presence of BMP-2 (30 ng/ml) (Figure 2.8D). Knockdown of p65 mRNA was confirmed at start and two hours in differentiation (left panel). Middle and right panels show Sox9 and COX-2 mRNA expression, respectively. As described above, Sox9 and COX-2 mRNA expression increased at two hours in differentiation and increased further with BMP-2 stimulation (see also Figure 2.8C). The BMP-2-initiated increased Sox9 and COX-2 mRNA upregulation is inhibited to equal levels as the differentiated control p65 knockdown condition without BMP-2 supplementation, supporting a role for p65 in this mechanism.

Finally we addressed whether BMP-2 might exert its pro-chondrogenic action early in differentiation through the NF-kB/p65 induced early Sox 9 expression. The early Sox 9 mRNA expression was targeted by a single Sox9 siRNA transfection (see also Figure 2.3E) and differentiation was initiated in the presence of BMP-2 (first 24 hours alone). As 
shown in Figure 2.8E, in the presence of BMP-2, Sox9 siRNA transfection resulted in an efficient knock-down of Sox9 mRNA and protein expression at two hours in differentiation. The early knock-down of Sox9 under BMP-2 treatment at two hours in differentiation resulted in impaired Col2a1 expression at seven days into differentiation.

Overall, these results suggest that BMP-2 action in the early chondrogenic phase of endochondral ossification may, in part, be explained via the herein described early transient $\mathrm{NF}-\kappa \mathrm{B} / \mathrm{p} 65$ activation and Sox 9 expression. These findings may provide a possible in vivo context for the herein described NF- $\mathrm{NB} / \mathrm{p} 65$ pathway.

A

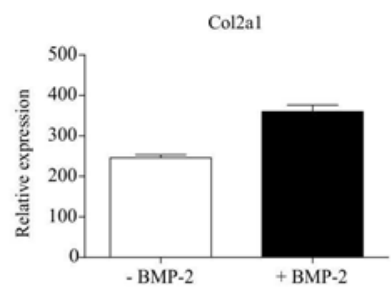

$\mathrm{C}$

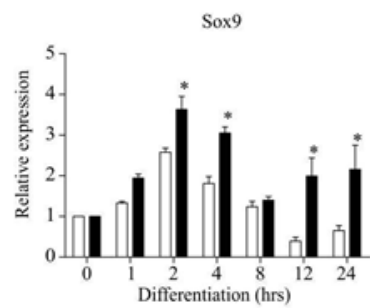

$\mathrm{D}$

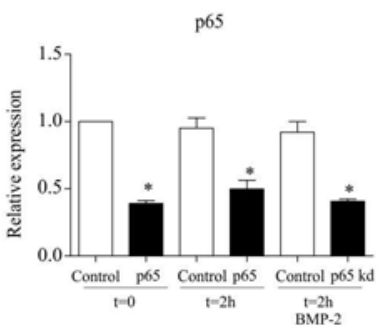

E

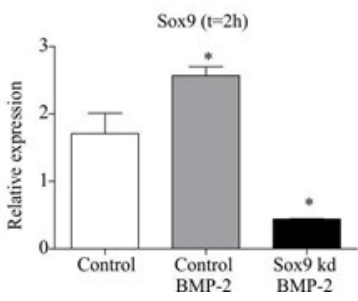

B
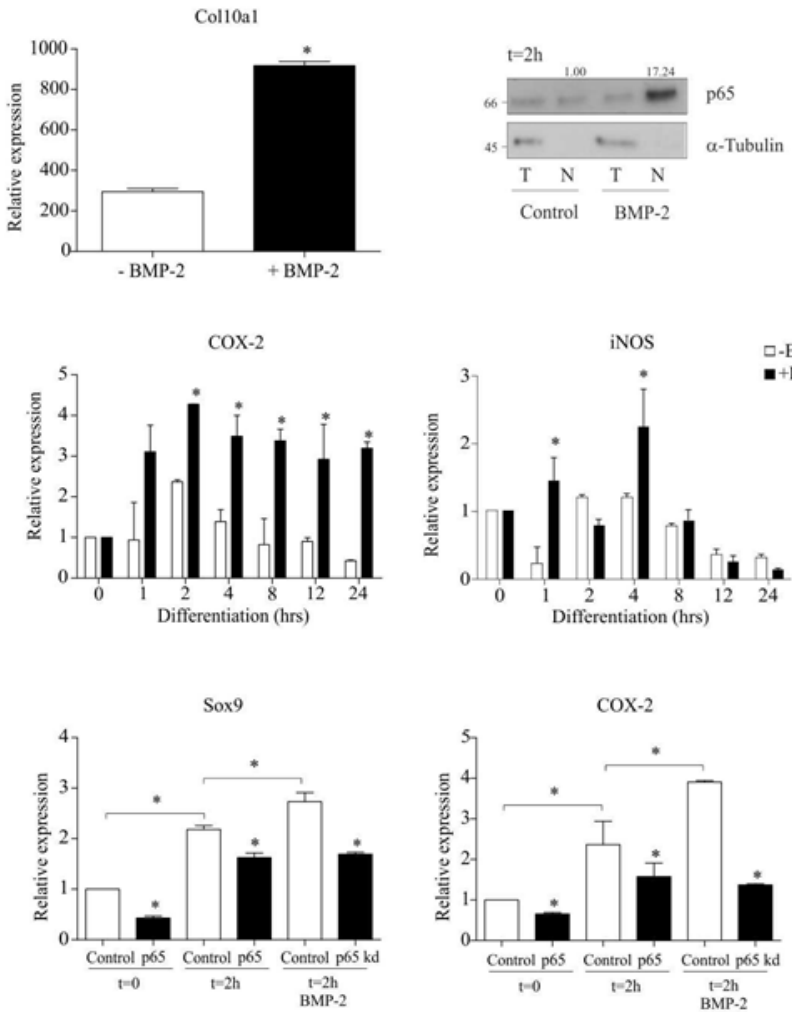

Col2al $(\mathrm{t}=7 \mathrm{~d})$
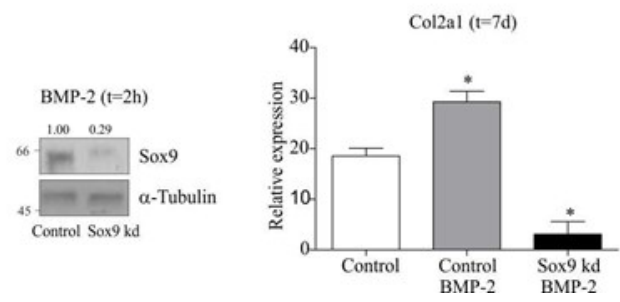
Figure 2.8: BMP-2 activates $N F-\kappa B / p 65$ in early ATDC5 chondrogenic differentiation

(A). ATDC5 was differentiated for 14 days in the absence or presence of BMP-2 $(30 \mathrm{ng} / \mathrm{ml})$. Relative mRNA expression of Col2a1 and Col10a1 was determined. (B). Cells were differentiated in the absence (control) or presence of BMP-2 $(30 \mathrm{ng} / \mathrm{ml})$ and total $(\mathrm{T})$ and nuclear $(\mathrm{N})$ fractions were prepared at 2 hours in differentiation. $\mathrm{NF}-\kappa \mathrm{B}$ was detected as p65. $(C)$. Cells were differentiated for 0-24 hours in the absence or presence of BMP-2

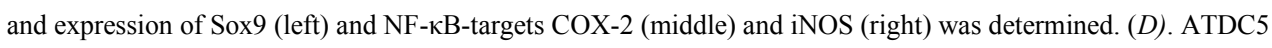
cells were transfected with a p65 siRNA duplex or control siRNA duplex and differentiated in the absence or presence of BMP-2 for 2 hours. Knock-down of p65 mRNA was confirmed at 0 and 2 hours in differentiation (left). Middle and right graphs show Sox9 and COX-2 mRNA expression, respectively. (E). Left panel set: Sox9 $\mathrm{KD}$ at mRNA and protein level at 2 hours in differentiation in cells transfected with scrambled (indicated as "Control") siRNAs, scrambled siRNAs in the presence of BMP-2, or Sox9 siRNAs (indicated as "Sox9") in the presence of BMP-2. Transfection was done the day prior to differentiation and BMP-2 treatment was for the first 24 hours only. Right panel: Col2a1 mRNA expression was determined in same experiment at day 7 in differentiation. $*=\mathrm{p}<0.05$.

\section{Discussion}

We here report that chondrogenic differentiation of chondroprogenitor cells is, at least in part, determined by early activation of NF- $\mathrm{kB} / \mathrm{p} 65$ which subsequently contributes to the initiation of chondrogenic differentiation by regulating the early expression of key chondrogenic factor Sox9.

Inflammatory mediators play crucial roles in cartilage degenerative conditions such as RA and $\mathrm{OA}^{30-32}$. Most, if not all of these inflammatory mediators are regulated via activated

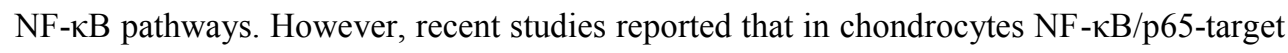
genes are not exclusively associated with cartilage degenerative conditions ${ }^{33}$. TNF $\alpha$ was recently described to regulate expression of BMP-2 via an NF- $\mathrm{BB} / \mathrm{p} 65$ dependent mechanism $^{8,34,35}$. Involvement of NF- $\kappa B$ was reported during development of the growth plate ${ }^{8,10}$, as well as in preventing apoptosis of maturating chondrocytes via interaction with $\mathrm{Nkx} 3.2^{9}$. $\mathrm{NF}-\kappa \mathrm{B} / \mathrm{p} 65$ has been reported to function as a transcription factor for Sox9 in mature chondrocytes $^{11}$ and finally, Aung and colleagues provided evidence that OA chondrocytes excrete soluble factors that initiate chondrogenic differentiation of human mesenchymal stem cells ${ }^{36}$. Except for the last study, most of these previous investigations were limited by the use of maturated chondrocytes, thereby leaving the question at which chondrogenic stage an imperative nuclear NF- $\mathrm{B} / \mathrm{p} 65$ presence would be required for differentiation. As we made use of cellular differentiation models that initiate chondrogenic differentiation 
from a progenitor stage onward, our experiments provided the possibility to address the role of NF- $\mathrm{kB} / \mathrm{p} 65$ activity in a specific chondrogenic phase, while at the same time determining the consequences of $\mathrm{NF}-\kappa \mathrm{B} / \mathrm{p} 65$ activity during subsequent later chondrogenic phases. We found that Sox9 induction during chondrogenic differentiation of chondroprogenitor cells is bi-phasic and is evident during the first hours of differentiation and induced for a second time later on in differentiation. The late Sox9 induction follows chondrocyte matrix expression and is thereby expected to transcriptionally regulate the induction of cartilage matrix genes such as Col2al and aggrecan ${ }^{15,16}$. In addition to late Sox9 expression, upon early chondrogenic differentiation NF- $\mathrm{BB} / \mathrm{p} 65$ transiently translocates to the nucleus, thereby enabling NF- $\mathrm{BB} / \mathrm{p} 65$-driven early Sox 9 transcription. Although the function of the novel short Sox9 pulse during the early start of chondrogenesis remains to be elucidated, our data indicate that it might function in the context of the Sox-trio $^{13,17}$ and is important in determining the chondrogenic outcome, possibly by priming the early differentiating cell for chondrogenic commitment by yet unknown (epigenetic) mechanisms.

Next to transcriptional induction of Sox 9 , the early chondrogenesis-associated NF- $\mathrm{BB} / \mathrm{p} 65$ activation results in additional transient expression of inflammatory target genes, such as COX-2, iNOS, Il-6 and TNF $\alpha$. Expression of these inflammatory NF- $\kappa B / p 65$ target genes may be an aspecific result of the transient activation of $\mathrm{NF}-\kappa \mathrm{B} / \mathrm{p} 65$. However ample experimental evidence supports a critical role for these inflammatory mediators in cellular fate determination in the context of the endochondral ossification during fracture healing, as the respective knock-out mice display a severely impaired fracture healing capacity ${ }^{6,37-46}$. Human mesenchymal stem cells (MSCs) have been reported to excrete several chondrogenic growth factors (e.g. insulin-like growth factor 1 (IGF-1) and fibroblast growth factor 2 (FGF-2)) upon inflammatory LPS or TNF $\alpha$ stimulation $^{47,48}$ and our data (Figure 2.9) show that treatment of differentiating ATDC5 cells with TNF $\alpha$, for the first 24 hours only, also resulted in significantly higher expression of chondrogenic growth factors (IGF-1, TGF- $\beta 1$, FGF-3, BMP-2, BMP-4) from seven days on in differentiation. It is therefore tempting to speculate that the expression of $\mathrm{NF}-\kappa \mathrm{B} / \mathrm{p} 65$-targets during the onset of chondrogenic differentiation may have an additional function in the paracrine signaling for later stages during endochondral ossification. Also, despite the degenerative environment, the endochondral formation of cartilaginous osteophytes is a hall mark of $\mathrm{OA}^{49}$. An aspect of their formation may also be found in NF- $\mathrm{BB} / \mathrm{p} 65$-driven chondrogenic differentiation of synovial or periosteal progenitors, initiated from the degenerating OA cartilage. As OA- 
IGF-1

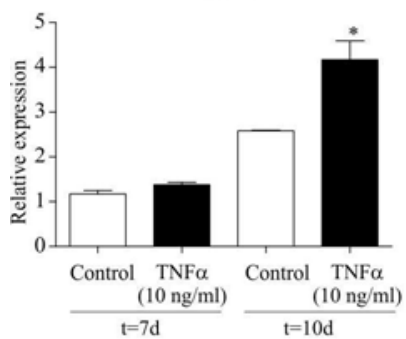

BMP-2

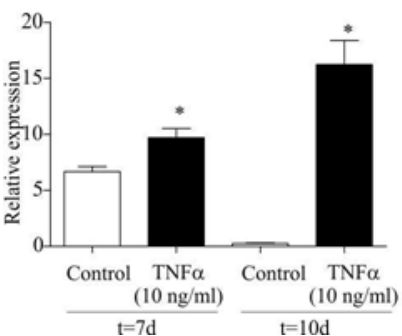

TGF- $\beta 1$

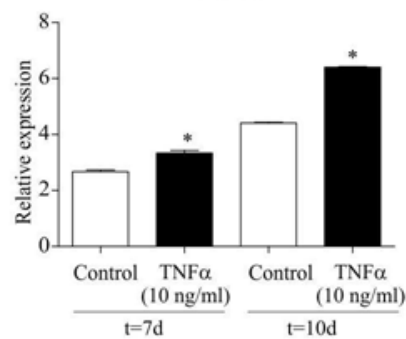

BMP-4

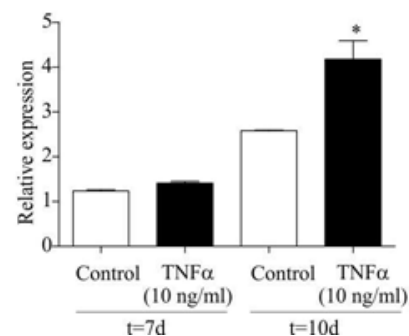

FGF-3

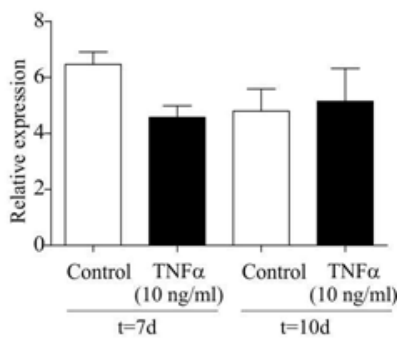

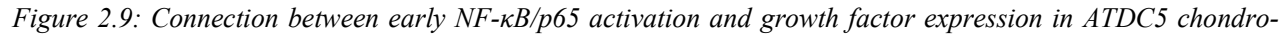
genic differentiation.

To investigate the connection between the early NF- $\mathrm{kB} / \mathrm{p} 65$ activation and expression of chondrogenic differentiation markers (see Figure 2.3D) at day 10 and 14, we determined the expression of multiple important chondrogenic growth factors. Addition of NF-kB-activator TNF $\alpha(10 \mathrm{ng} / \mathrm{ml})$ for the first 24 hours resulted in increased expression of the chondrogenic growth factors at day 7 and/or 10 in differentiation (except for FGF3), consistent with the data from Figure 2.3.

like conditions are absent in the developing growth plate, expression of $\mathrm{NF}-\kappa \mathrm{B} / \mathrm{p} 65$-targets and growth factors by differentiating growth plate chondrocytes may maintain growth plate chondrogenic differentiation of local resting zone progenitor cells in a similar paracrine fashion. Although in early chondrogenic differentiation $\mathrm{NF}-\kappa \mathrm{B} / \mathrm{p} 65$ is clearly activated during the first hours in the differentiation process, we do not yet fully understand how the chondrogenic culture environment triggers this inflammatory response. Several chondrogenic growth factors are associated with $\mathrm{NF}-\kappa \mathrm{B} / \mathrm{p} 65$ signaling. It is known that TGF- $\beta$-receptor (TGFR) and IGF-receptor (IGFR) signaling activate NF- $\kappa \mathrm{B}$ and expression of chondrogenic markers in chondrocytes ${ }^{50,51}$. Key extracellular signaling molecules triggering chondrogenesis in vitro are insulin and TGF- $\beta$. Hence, insulin/IGFRand TGF- $\beta /$ TGFR-activation likely initiate signaling through $\mathrm{NF}-\kappa \mathrm{B} / \mathrm{p} 65$, resulting in initiation of early transient Sox9 induction. Our observation that stimulation of NF- $\mathrm{BB}$ activity by LPS or TNF $\alpha$ under chondrogenic conditions or even under proliferation 
conditions (in the absence of standard chondrogenic stimuli) enhances or triggers Sox9 expression and eventually contributes to the chondrogenic potential, is well in line with this notion. Although mesenchymal progenitor cells express TNFR and TLR-2/4 ${ }^{52,53}$, the use of LPS and TNF $\alpha$ to activate NF-kB might be contradictory in the context of chondrogenic differentiation. However, these agents were solely used as NF-KB-activating tools in the herein described work. More relevant to the in vivo context of early chondrogenic differentiation and endochondral ossification BMP-2 has previously been described to be able to translocate NF-KB/p65 to the nucleus ${ }^{29}$ and to be involved in Sox9 regulation during early mesenchymal condensation ${ }^{28}$. These previous findings may provide an in vivo context in which the herein described BMP-2 mediated NF-KB/p65 driven early Sox 9 expression may function. In addition, other studies ${ }^{8,10}$ have shown that BMP-2 expression itself can be regulated by $\mathrm{NF}-\mathrm{KB} / \mathrm{p} 65$ during late chondrogenesis in maturated chondrocytes, thereby contributing to longitudinal bone growth and preventing apoptosis of these chondrocytes. Therefore, our and previous findings indicate that BMP-2 action and regulation might depend on the chondrogenic differentiation status ${ }^{34}$.

In conclusion, our data indicate that initiation of chondrogenic differentiation during endochondral development, at least in part, depends on an early activation of $\mathrm{NF}-\kappa \mathrm{B} / \mathrm{p} 65$. The early NF- $\mathrm{kB} / \mathrm{p} 65$ activation evokes a novel early and transient expression of Sox 9 , which, together with a late Sox9 induction, contributes to the outcome of the chondrogenic differentiation program of mesenchymal progenitor cells. Our findings complement previously reported NF- $\mathrm{KB} / \mathrm{p} 65$ involvement in chondrogenic differentiation and provide novel insight into the origin, timing and dynamics of NF- $\mathrm{kB} / \mathrm{p} 65$-induced gene expression in early chondrogenic differentiation. These data add to an emerging and growing concept ${ }^{7}$ where differentiating chondrocytes and endochondral development are regulated by $\mathrm{NF}-\mathrm{\kappa B} / \mathrm{p} 65$-mediated processes and may be used as new leads to modulate chondrogenic differentiation in cartilage and bone regenerative medicine approaches such as the ACT technique ${ }^{54}$ and the in vivo bioreactor technique ${ }^{55,56}$. 


\section{Acknouledgements}

This work is financially supported the Dutch Arthritis Association (grant LLP14) and the Dutch Stichting Annafonds|NOREF (grants 07/07 and 08/42). LPS and surplus mice were provided by Dr. M. Poeze and Drs. N. Wijnands (Dept. of General Surgery, MUMC, the Netherlands) and Parthenolide by Dr. R. Langen (Dept. of Pulmonology, MUMC, the Netherlands). P.T. Oostveen (Dept. of Orthopaedic Surgery, MUMC, the Netherlands) for performing cPAC experiments. Helpful suggestions about the $\mathrm{cPAC}$ model were provided by Dr. C.C. van Donkelaar (Dept. of Orthopaedic Biomechanics, Eindhoven University of Technology, Eindhoven, the Netherlands).

\section{Supplemental Figures}

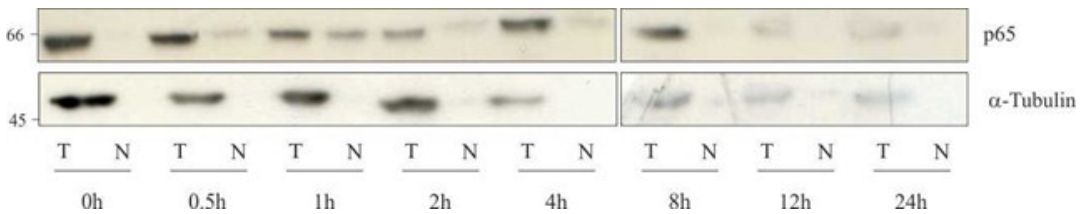

Figure S2.1: $N F-\kappa B / p 65$ nuclear translocation during early ATDC5 differentiation. In addition to Figure 2.1A, nuclear translocation of p65 was besides at 0, 0.5, 1 and 2 hours in differentiation also determined at 4, 8, 12 and 24 hours in differentiation of ATDC5 cells. Total extract (T) and nuclear (N) fractions were isolated. Cytoplasmic marker: $\alpha$-tubulin. $\mathrm{NF}-\kappa \mathrm{B} / \mathrm{p} 65$ is transiently activated at $0.5-4$ hours in differentiation. 


\section{References}

1 Kronenberg, H. M. Developmental regulation of the growth plate. Nature 423, 332-336 (2003).

2 Mackie, E. J., Ahmed, Y. A., Tatarczuch, L., Chen, K. S. \& Mirams, M. Endochondral ossification: how cartilage is converted into bone in the developing skeleton. Int J Biochem Cell Biol 40, 46-62 (2008).

3 Zuscik, M. J., Hilton, M. J., Zhang, X., Chen, D. \& O'Keefe, R. J. Regulation of chondrogenesis and chondrocyte differentiation by stress. $J$ Clin Invest 118, 429438 (2008).

4 Grundnes, O. \& Reikeras, O. The importance of the hematoma for fracture healing in rats. Acta Orthop Scand 64, 340-342 (1993).

5 Einhorn, T. A., Majeska, R. J., Rush, E. B., Levine, P. M. \& Horowitz, M. C. The expression of cytokine activity by fracture callus. $J$ Bone Miner Res 10, 12721281, doi:10.1002/jbmr.5650100818 (1995).

6 Mountziaris, P. M. \& Mikos, A. G. Modulation of the Inflammatory Response for Enhanced Bone Tissue Regeneration. Tissue Eng Part B Rev (2008).

7 Novack, D. V. Role of NF-kappaB in the skeleton. Cell Res 21, 169-182, doi:cr2010159 [pii] 10.1038/cr.2010.159.

8 Feng, J. Q. et al. NF-kappaB specifically activates BMP-2 gene expression in growth plate chondrocytes in vivo and in a chondrocyte cell line in vitro. $J$ Biol Chem 278, 29130-29135 (2003).

9 Park, M. et al. Constitutive RelA activation mediated by Nkx3.2 controls chondrocyte viability. Nat Cell Biol 9, 287-298 (2007).

10 Wu, S., Flint, J. K., Rezvani, G. \& De Luca, F. Nuclear factor-kappaB p65 facilitates longitudinal bone growth by inducing growth plate chondrocyte proliferation and differentiation and by preventing apoptosis. J Biol Chem 282, 33698-33706 (2007).

11 Ushita, M. et al. Transcriptional induction of SOX9 by NF-kappaB family member RelA in chondrogenic cells. Osteoarthritis and cartilage / OARS, Osteoarthritis Research Society 17, 1065-1075, doi:10.1016/j.joca.2009.02.003 (2009).

12 Akiyama, H., Chaboissier, M. C., Martin, J. F., Schedl, A. \& de Crombrugghe, B. The transcription factor Sox9 has essential roles in successive steps of the chondrocyte differentiation pathway and is required for expression of Sox 5 and Sox6. Genes Dev 16, 2813-2828 (2002).

13 Lefebvre, V., Behringer, R. R. \& de Crombrugghe, B. L-Sox5, Sox6 and Sox9 control essential steps of the chondrocyte differentiation pathway. Osteoarthritis Cartilage 9 Suppl A, S69-75 (2001).

14 Lefebvre, V. \& de Crombrugghe, B. Toward understanding SOX9 function in chondrocyte differentiation. Matrix Biol 16, 529-540 (1998).

15 Han, Y. \& Lefebvre, V. L-Sox 5 and Sox6 drive expression of the aggrecan gene in cartilage by securing binding of Sox 9 to a far-upstream enhancer. Mol Cell Biol 28, 4999-5013, doi:MCB.00695-08 [pii] 10.1128/MCB.00695-08 (2008).

16 Lefebvre, V., Huang, W., Harley, V. R., Goodfellow, P. N. \& de Crombrugghe, B. SOX9 is a potent activator of the chondrocyte-specific enhancer of the pro alpha1(II) collagen gene. Mol Cell Biol 17, 2336-2346 (1997). 
17 Lefebvre, V., Li, P. \& de Crombrugghe, B. A new long form of Sox5 (L-Sox5), Sox6 and Sox9 are coexpressed in chondrogenesis and cooperatively activate the type II collagen gene. Embo $J$ 17, 5718-5733, doi:10.1093/emboj/17.19.5718 (1998).

18 Atsumi, T., Miwa, Y., Kimata, K. \& Ikawa, Y. A chondrogenic cell line derived from a differentiating culture of AT805 teratocarcinoma cells. Cell Differ Dev 30, 109-116 (1990).

19 Schallmoser, K. et al. Rapid large-scale expansion of functional mesenchymal stem cells from unmanipulated bone marrow without animal serum. Tissue engineering 14, 185-196 (2008).

20 Kock, L. M. et al. Tuning the differentiation of periosteum-derived cartilage using biochemical and mechanical stimulations. Osteoarthritis Cartilage 18, 15281535, doi:S1063-4584(10)00298-0 [pii]

10.1016/j.joca.2010.09.001 (2010).

21 O'Driscoll, S. W., Recklies, A. D. \& Poole, A. R. Chondrogenesis in periosteal explants. An organ culture model for in vitro study. J Bone Joint Surg Am 76, 1042-1051 (1994).

22 Chen, L., Fink, T., Zhang, X. Y., Ebbesen, P. \& Zachar, V. Quantitative transcriptional profiling of ATDC5 mouse progenitor cells during chondrogenesis. Differentiation 73, 350-363 (2005).

23 Hayden, M. S. \& Ghosh, S. Shared principles in NF-kappaB signaling. Cell 132, 344-362 (2008).

24 Abad, V. et al. The role of the resting zone in growth plate chondrogenesis. Endocrinology 143, 1851-1857 (2002).

25 Huang, W., Zhou, X., Lefebvre, V. \& de Crombrugghe, B. Phosphorylation of SOX9 by cyclic AMP-dependent protein kinase A enhances SOX9's ability to transactivate a Col2a1 chondrocyte-specific enhancer. Mol Cell Biol 20, 41494158 (2000).

26 Quandt, K., Frech, K., Karas, H., Wingender, E. \& Werner, T. MatInd and MatInspector: new fast and versatile tools for detection of consensus matches in nucleotide sequence data. Nucleic Acids Res 23, 4878-4884, doi:5s0483 [pii] (1995).

27 Ushita, M. et al. Transcriptional induction of SOX9 by NF-kappaB family member RelA in chondrogenic cells. Osteoarthritis Cartilage (2009).

28 Yoon, B. S. \& Lyons, K. M. Multiple functions of BMPs in chondrogenesis. Journal of cellular biochemistry 93, 93-103 (2004).

29 Sugimori, K. et al. BMP-2 prevents apoptosis of the N1511 chondrocytic cell line through PI3K/Akt-mediated NF-kappaB activation. $J$ Bone Miner Metab 23, 411419, doi:10.1007/s00774-005-0622-7 (2005).

30 Glasson, S. S. et al. Deletion of active ADAMTS5 prevents cartilage degradation in a murine model of osteoarthritis. Nature 434, 644-648, doi:nature03369 [pii] 10.1038/nature03369 (2005).

31 Romas, E., Gillespie, M. T. \& Martin, T. J. Involvement of receptor activator of NFkappaB ligand and tumor necrosis factor-alpha in bone destruction in rheumatoid arthritis. Bone 30, 340-346, doi:S8756328201006822 [pii] (2002).

32 Wehling, N. et al. Interleukin-1beta and tumor necrosis factor alpha inhibit chondrogenesis by human mesenchymal stem cells through NF-kappaB-dependent pathways. Arthritis Rheum 60, 801-812 (2009).

33 Ulivi, V., Giannoni, P., Gentili, C., Cancedda, R. \& Descalzi, F. p38/NF-kBdependent expression of COX-2 during differentiation and inflammatory response of chondrocytes. Journal of cellular biochemistry 104, 1393-1406 (2008).

34 Fukui, N. et al. Pro-inflammatory cytokine tumor necrosis factor-alpha induces 
bone morphogenetic protein-2 in chondrocytes via mRNA stabilization and transcriptional up-regulation. J Biol Chem 281, 27229-27241 (2006).

Fukui, N., Zhu, Y., Maloney, W. J., Clohisy, J. \& Sandell, L. J. Stimulation of BMP-2 expression by pro-inflammatory cytokines IL-1 and TNF-alpha in normal and osteoarthritic chondrocytes. J Bone Joint Surg Am 85-A Suppl 3, 59-66 (2003).

36 Aung, A., Gupta, G., Majid, G. \& Varghese, S. Osteoarthritic chondrocytesecreted morphogens induce chondrogenic differentiation of human mesenchymal stem cells. Arthritis Rheum 63, 148-158, doi:10.1002/art.30086 (2011).

Baldik, Y. et al. Deletion of iNOS gene impairs mouse fracture healing. Bone 37, 32-36 (2005).

Diwan, A. D., Wang, M. X., Jang, D., Zhu, W. \& Murrell, G. A. Nitric oxide modulates fracture healing. J Bone Miner Res 15, 342-351 (2000).

Franchimont, N., Durant, D. \& Canalis, E. Interleukin-6 and its soluble receptor regulate the expression of insulin-like growth factor binding protein-5 in osteoblast cultures. Endocrinology 138, 3380-3386 (1997).

Gerstenfeld, L. C. et al. Impaired intramembranous bone formation during bone repair in the absence of tumor necrosis factor-alpha signaling. Cells Tissues Organs 169, 285-294 (2001).

1 Gerstenfeld, L. C. et al. Impaired fracture healing in the absence of TNF-alpha signaling: the role of TNF-alpha in endochondral cartilage resorption. $J$ Bone Miner Res 18, 1584-1592 (2003).

Naik, A. A. et al. Reduced COX-2 expression in aged mice is associated with impaired fracture healing. J Bone Miner Res 24, 251-264 (2009).

Rundle, C. H. et al. Microarray analysis of gene expression during the inflammation and endochondral bone formation stages of rat femur fracture repair. Bone 38, 521-529 (2006).

44 Simon, A. M. \& O'Connor, J. P. Dose and time-dependent effects of cyclooxygenase-2 inhibition on fracture-healing. J Bone Joint Surg Am 89, 500-511 (2007).

45 Zhang, X. et al. Cyclooxygenase-2 regulates mesenchymal cell differentiation into the osteoblast lineage and is critically involved in bone repair. $J$ Clin Invest 109, 1405-1415 (2002).

46 Zhu, W., Murrell, G. A., Lin, J., Gardiner, E. M. \& Diwan, A. D. Localization of nitric oxide synthases during fracture healing. J Bone Miner Res 17, 1470-1477 (2002).

47 Crisostomo, P. R. et al. Human mesenchymal stem cells stimulated by TNFalpha, LPS, or hypoxia produce growth factors by an NF kappa B- but not JNKdependent mechanism. American journal of physiology 294, C675-682 (2008).

Wang, M., Crisostomo, P. R., Herring, C., Meldrum, K. K. \& Meldrum, D. R. Human progenitor cells from bone marrow or adipose tissue produce VEGF, HGF, and IGF-I in response to TNF by a p38 MAPK-dependent mechanism. American journal of physiology 291, R880-884 (2006).

49 van der Kraan, P. M. \& van den Berg, W. B. Osteophytes: relevance and biology. Osteoarthritis Cartilage 15, 237-244, doi:S1063-4584(06)00327-X [pii] 10.1016/j.joca.2006.11.006 (2007).

50 Grau, A. M., Datta, P. K., Zi, J., Halder, S. K. \& Beauchamp, R. D. Role of Smad proteins in the regulation of NF-kappaB by TGF-beta in colon cancer cells. Cell Signal 18, 1041-1050, doi:S0898-6568(05)00235-4 [pii] 10.1016/j.cellsig.2005.08.021 (2006).

51 Wu, S., Fadoju, D., Rezvani, G. \& De Luca, F. Stimulatory effects of insulin-like 
growth factor-I on growth plate chondrogenesis are mediated by nuclear factorkappaB p65. J Biol Chem 283, 34037-34044 (2008).

52 Croitoru-Lamoury, J., Lamoury, F. M., Zaunders, J. J., Veas, L. A. \& Brew, B. J. Human mesenchymal stem cells constitutively express chemokines and chemokine receptors that can be upregulated by cytokines, IFN-beta, and Copaxone. $J$ Interferon Cytokine Res 27, 53-64, doi:10.1089/jir.2007.0037 (2007).

53 Pevsner-Fischer, M. et al. Toll-like receptors and their ligands control mesenchymal stem cell functions. Blood 109, 1422-1432, doi:10.1182/blood-2006-06028704 (2007).

54 Brittberg, M. et al. Treatment of deep cartilage defects in the knee with autologous chondrocyte transplantation. N Engl J Med 331, 889-895 (1994).

55 Emans, P. J. et al. Autologous engineering of cartilage. Proc Natl Acad Sci U S A 107, 3418-3423, doi:0907774107 [pii]10.1073/pnas.0907774107 (2010).

56 Stevens, M. M. et al. In vivo engineering of organs: the bone bioreactor. Proc Natl Acad Sci U S A 102, 11450-11455 (2005). 


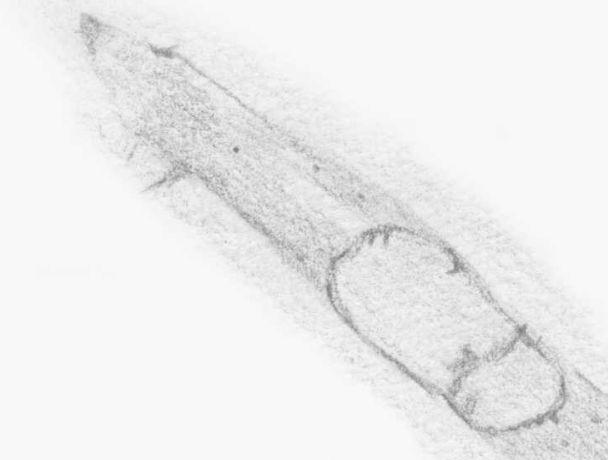

Chapter 3

\section{Osmolarity Determines the in vitro Chondrogenic Differentiation}

\section{Capacity of Progenitor Cells via Nuclear Factor of Activated}

\section{$\tau_{\text {-cells } 5}$}

\section{Adapted from:}

Osmolarity Determines the in vitro Chondrogenic Differentiation Capacity of Progenitor Cells via Nuclear Factor of Activated T-cells 5

Marjolein M.J. Caron ${ }^{1}$, Anna E. van der Windt ${ }^{2}$, Pieter JEmans ${ }^{1}$, Lodewijk W. van Rhijn ${ }^{1}$, Holger Jahr ${ }^{2 \#}$ and Tim $^{2}$ J. M. Welting ${ }^{1 \#}$

${ }^{1}$ Department of Orthopaedic Surgery, Maastricht University Medical Center, Maastricht, the Netherlands ${ }^{2}$ Department of Orthopaedics, Erasmus MC, University Medical Center Rotterdam, Rotterdam, the Netherlands \#Contributed equally

Bone. (2013); 53(1): p.94-102 


\section{Abstract}

Introduction: Previous studies have shown that human articular chondrocytes in vitro are osmolarity-responsive and increase matrix synthesis under cartilage-specific physiological osmolarity. The effects of increased osmolarity on chondrogenesis of progenitor cells in vitro are largely unknown. We therefore aimed to elucidate whether hyperosmolarity facilitates their chondrogenic differentiation and whether Nfat5 is involved.

Methodology: ATDC5 cells and human bone marrow stem cells (hBMSCs) were differentiated in the chondrogenic lineage in control and increased osmolarity conditions. Chondrogenic outcome was measured by gene- and protein expression analysis. RNAi was used to determine the role of Nfat5 in chondrogenic differentiation under normal and increased osmolarity.

Results: Increasing the osmolarity of differentiation medium with $100 \mathrm{mOsm}$ resulted in significantly increased chondrogenic marker expression (Col2a1, Col10a1, Acan, Sox9, Runx2 and GAGs) during chondrogenic differentiation of the two chondroprogenitors, ATDC5 and hBMSCs. Nfat5 knockdown under both control and increased osmolarity affected chondrogenic differentiation and suppressed the osmolarity-induced chondrogenic induction. Knockdown of Nfat5 in early differentiation significantly decreased early Sox 9 expression, whereas knockdown of Sox9 in early differentiation did not affect early Nfat5 expression.

Conclusions: Increasing the osmolarity of chondrogenic culture media by $100 \mathrm{mOsm}$ significantly increased chondrogenic gene expression during the course of chondrogenic differentiation of progenitor cells. Nfat5 may be involved in regulating chondrogenic differentiation of these cells under both normal and increased osmolarities and might regulate chondrogenic differentiation through influencing early Sox9 expression. 


\section{Introduction}

Chondrogenic differentiation of progenitor cells plays an essential role during endochondral ossification for skeletal growth and bone fracture healing ${ }^{1,2}$. Moreover, progenitor cell -based cartilage regeneration is a rapidly evolving field and methodologies promoting chondrogenic differentiation of mesenchymal progenitor and stem cells to chondrocytes are of interest to improve cartilage and endochondral bone regenerative medicine approaches.

Chondrogenic differentiation during endochondral ossification is a multi-step developmental process during which mesenchymal progenitor cells condensate, differentiate into extracellular matrix (ECM) producing chondrocytes and ultimately terminally differentiate into hypertrophic chondrocytes ${ }^{1,3}$. This process is accompanied by a stage-dependent expression of chondrogenic makers: while for example Sox9 (SRY (sex determining region Y)-box 9) is a primary determinant of differentiation from early stages onwards ${ }^{4,5}$, its transcriptional targets collagen type II (Col2a1) and aggrecan (Acan) are prominently expressed by more mature, ECM producing chondrocytes. Collagen type X (Col10a1) and its main transcription factor Runx2 (Runt-related transcription factor 2) are specifically expressed by hypertrophic differentiating chondrocytes ${ }^{4,5}$.

The large amount of ECM produced by chondrocytes is mainly composed of proteoglycans (PG; like aggrecan) which are entangled in a network of collagens (mainly Col2a1) and further contains a lot of matrix-retained water ${ }^{6}$. The glycosaminoglycan (GAG) side chains of the PGs are sulphated and responsible for a high fixed negative charge density, which attracts mobile cations and water from the ECM-environment ${ }^{7,8}$. This, together with the quality of the collagen network, determines the osmolarity of the extracellular fluid and provides strength and flexibility to the tissue. The extracellular osmolarity of healthy articular cartilage ranges between 350 and $480 \mathrm{mOsm}$ and is thus markedly higher than that of standard culture medium ${ }^{9,10}$, which ranges around plasma levels $(280 \mathrm{mOsm})^{11,12}$. Several studies have shown that articular chondrocytes in vitro are osmolarity-responsive and increase their ECM synthesis under chondrocyte-physiological osmolarity ${ }^{10,13-15}$. The nuclear factor of activated T-cells 5 (Nfat5/TonEBP) plays an important role in this response $^{14}$. Nfat5 is a member of the Rel family of transcription factors ${ }^{16}$ and mediates transcriptional activation of ion transporters like the sodium/myo-inositol transporter encoded by Slc5a3 $3^{17,18}$ and calcium binding proteins like $\mathrm{S} 100 \mathrm{a} 4^{19,20}$ upon hypertonic stress in several cell types, including human articular chondrocytes ${ }^{14}$. Collectively, the 
Nfat5-activated transporters exchange intracellular ions for compatible osmolytes and thereby regulate intracellular ionic strength and cell volume upon hypertonic stress ${ }^{21}$. However, a growing body of evidence indicates that Nfat5 can also be regulated independently of osmolarity ${ }^{22}$ and has diverse osmolarity-independent transcriptional functions in cell proliferation/survival ${ }^{23}$, carcinogenesis $^{24}$ and many others. To the best of our knowledge, no literature is available describing the effects of increased osmolarity on chondrogenic differentiation of progenitor cells in vitro. In addition, the expression and a potential (osmolarity-independent) function of Nfat5 during chondrogenic differentiation remains elusive. We therefore hypothesized that Nfat5 is involved in in vitro chondrogenic differentiation of progenitor cells during endochondral ossification and that the differentiation process can be enhanced by increasing the osmolarity of the culture medium possibly via an Nfat5 dependent mechanism.

In the present study we used ATDC5 cells and human bone marrow stem cells (hBMSCs) as in vitro models for chondrogenic differentiation and provide evidence that Nfat5 expression is indeed regulated during chondrogenic differentiation independently of osmolarity status, but also responds to increased osmolarity during chondrogenic differentiation. Furthermore, we show that increased osmolarity improves chondrogenic differentiation of progenitor cells in vitro and that, at least in part, this is regulated via Nfat5-mediated transcriptional regulation of Sox9.

\section{Materials and Methods}

\subsection{ATDC5 cell culture}

ATDC5 cells were cultured in proliferation medium under a humidified atmosphere at $37^{\circ} \mathrm{C}, 5 \% \mathrm{CO}_{2}$. Proliferation medium consisted of DMEM/F12 (Invitrogen, Carlsbad, CA, USA), 5\% FCS (PAA, Pasching, Austria), 1\% antibiotic/antimycotic (mixture of 10,000 U/ $\mathrm{ml}$ penicillin, 10,000 $\mu \mathrm{g} / \mathrm{ml}$ streptomycin and $25 \mu \mathrm{g} / \mathrm{ml}$ amphotericin B; Invitrogen) and 1\% NEAA (non-essential amino acids; Invitrogen) and was changed every two days. Differentiation medium comprised proliferation medium supplemented with $10 \mu \mathrm{g} / \mathrm{ml}$ insulin (Sigma, St. Louis, MO, USA), $10 \mu \mathrm{g} / \mathrm{ml}$ transferrin (Roche Applied Science, Indianapolis, IN, USA) and $30 \mathrm{nM}$ sodium selenite (Sigma, St. Louis, MO, USA). Cells were plated at approximately 6400 cells $/ \mathrm{cm}^{2}$ in cell culture dishes, allowed to adhere over- 
night and the following day chondrogenesis was initiated by changing the proliferation medium to differentiation medium. Differentiation medium was changed every two days (day 0-10) and daily (from day 10 on). The osmolarity of the ATDC5 differentiation medium was determined to $310 \pm 5$ mOsm using an Osmomat 030 (Gonotec GmbH, Germany). The osmolarity of this medium was increased with 100 or $200 \mathrm{mOsm}$, respectively, by adding sterile $\mathrm{NaCl}$ from day 0 in differentiation. For RNAi-experiments an Nfat5 siRNA-duplex (sense: 5'-CCAGUUCCUACAAUGAUAACACU-3', antisense: 5'-AGUGUU AUCAUUGUAGGAACUGG-3'), a Sox9 siRNA duplex (sense: 5'- GACUCACAUCUCUCCUAAUTT-3', anti-sense: 5'- AUUAGGAGAGAUGUGAGUCTT-3') and a scrambled siRNA-duplex (indicated by "Mock") were used (Eurogentec). ATDC5 cells were seeded at approximately 25,000 cells $/ \mathrm{cm}^{2}$ and transfection with siRNAs $(30 \mathrm{nM})$ was performed using ICAfectin 442 (Eurogentec) according to manufacturers' protocol. Knockdown was allowed for 1 day prior to the initiation of chondrogenic differentiation and knockdown for longer timeframes was accomplished by retransfection of the respective siRNA duplex.

\section{2 hBMSC isolation and culture}

Human bone marrow mesenchymal stem cells were obtained from iliac crest bone marrow aspirate from young, genetically healthy individuals (Medical ethical committee MUMC approval 08-4-056). BMSCs were isolated using Ficoll-Paque PLUS (Amersham Pharmacia, Diegem, Belgium) and plated on culture plates. Unbound cells were removed after 48 hours, and bound fraction was expanded in culture medium to five passages under a humidified atmosphere at $37^{\circ} \mathrm{C}, 5 \% \mathrm{CO}_{2}$. Culture medium consisted of DMEM high glucose (Invitrogen), 10\% FCS (PAA), 1\% antibiotic/antimycotic and 1\% NEAA. Chondrogenesis was performed in monolayer by plating the cells at approximately 30,000 cells/ $\mathrm{cm}^{2}$ one day prior to initiating chondrogenesis with differentiation medium (proliferation medium supplemented with 1\% ITS (Invitrogen), $50 \mathrm{mg} / \mathrm{ml} \mathrm{L-ascorbic} \mathrm{acid-2-phosphate}$ (Sigma) and $1 \mathrm{ng} / \mathrm{ml}$ TGF $\beta 3(\mathrm{R} \& \mathrm{D}))^{25}$. The osmolarity of the differentiation medium was determined using an Osmomat 030 (Gonotec GmbH, Germany) and was measured to be $285 \pm 5$ mOsm. When indicated, osmolarity of the differentiation medium was increased with $100 \mathrm{mOsm}$ by adding sterile $\mathrm{NaCl}$ from day 0 in differentiation. Throughout the hBMSC differentiation, medium was changed every two days. 


\subsection{HAC isolation and culture}

Chondrocytes were obtained from the unaffected regions of osteoarthritic (OA) cartilage from total knee arthroplasty (MEC approval 08-4-028). Cartilage was separated from the subchondral bone and cut into small pieces using a sterile surgical blade. Cartilage pieces were digested overnight at $37^{\circ} \mathrm{C}$ in collagenase type II solution (300 U/ml in HEPES buffered DMEM/F12 (Invitrogen, Carlsbad, CA, USA) supplemented with antibiotics) under continuous agitation. The preparation was rinsed with $0.9 \% \mathrm{NaCl}$ over a $70 \mu \mathrm{m}$ cell strainer and plated in culture flasks. Cells were cultured in a humidified atmosphere at $37^{\circ} \mathrm{C}, 5 \%$ $\mathrm{CO}_{2}$ and after reaching confluency the cells were continuously passaged 1:2 until passage 5. Culture medium consisted of DMEM/F12 (Invitrogen), 10\% FCS (PAA; Pasching, Austria), $1 \%$ antibiotic/antimycotic (Invitrogen) and 1\% non-essential amino acids (NEAA; Invitrogen). Chondrogenic redifferentiation was performed with passage five cells from six individuals and each isolate was redifferentiated in monolayer culture. The cells were plated 1 day prior to start of differentiation at a density close to confluency $(30,000$ cells $/ \mathrm{cm}^{2}$ ) to mimic the high cell density known from 3D cultures. Chondrogenic differentiation was initiated by changing to differentiation medium (DMEM/F12, $1 \%$ antibiotic/ antimycotic and 1\% NEAA supplemented with 1\% ITS (insulin-transferrin-sodium selenite media supplement, Invitrogen), 1\% L-ascorbic acid-2-phosphate (Sigma Aldrich, St. Louis, MO, USA) and $10 \mathrm{ng} / \mathrm{ml}$ TGF- $\beta 3$ (R\&D, Minneapolis, MN, USA)). Differentiation medium was changed every two days. Cells were harvested for RNA and protein analysis at day 7 in redifferentiation (see below).

\section{$\underline{2.4 R T-q P C R}$}

For RNA isolation, cells were washed 3 times with $0.9 \% \mathrm{NaCl} w / \mathrm{v}$ and disrupted with 500 $\mu 1$ Trizol (Invitrogen). RNA isolation, RNA quantification by UV-spectrometry (Nanodrop, Thermo Scientific), and cDNA synthesis were performed as described before ${ }^{26,27}$. Real time quantitative PCR (RT-qPCR) was performed using Mesagreen qPCR mastermix plus for SYBR ${ }^{\circledR}$ Green (Eurogentec, Seraing, Belgium). An Applied Biosystems ABI PRISM 7700 Sequence Detection System was used for amplification using the following protocol: initial denaturation at $95^{\circ} \mathrm{C}$ for 10 minutes, followed by 40 cycles of DNA amplification (denaturing for 15 seconds at $95^{\circ} \mathrm{C}$ and annealing for 1 minute at $60^{\circ} \mathrm{C}$ ) followed by a dissociation curve. Data were analysed using the standard curve method, mRNA expression was normalized to a reference gene ( $\beta$-actin for ATDC5 and 28S rRNA for hBMSCs) and gene expression was calculated as fold change as com- 
pared to day 0 ( $\mathrm{t}=0$ in graphs). Primer sequences are depicted in Table 3.1.

Table 3.1. Primer sequences for RTqPCR

\begin{tabular}{lll}
\hline Oligo sets mouse & $\underline{\text { Forward }}$ & $\underline{\text { Reverse }}$ \\
\hline Col2a1 & '5-TGGGTGTTCTATTTATTTATTGTCTTCC-3' & '5-GCGTTGGACTCACACCAGTTAGT-3' \\
Col10a1 & '5-CATGCCTGATGGCTTCATAAA-3' & '5-AAGCAGACACGGGCATACCT-3' \\
Runx2 & '5-CGATGAAGACCCCAACCCTAA-3' & '5-ACTGGTAATGGCATCAAGGGATA-3' \\
Sox9 & '5-AGTACCCGCACCTGCACAAC-3' & '5-TACTTGTAGTCCGGGTGGTCTTTC-3' \\
Acan & '5-CATGAGAGAGGCGAATGGAA-3 & '5-TGATCTCGTAGCGATCTTTCTTCT-3' \\
Nfat5 & '5-GGGTCAAACGACGAGATTGTG-3 & '5-TTGTCCGTGGTAAGCTGAGAA-3' \\
Scl5a3 & '5-CACTTCTGTCATTGGAGCGCT-3' & '5-ATGGCAATGTCTGCTGTGTCC-3' \\
S100a4 & '5-GTCCACCTTCCACAAGTACTCG-3' & '5-TCATCTGTCCTTTTCCCCAAG-3' \\
B-Actin & '5-GACAGGATGCAGAAGGAGATTACTG-3' & '5-CCACCGATCCACACAGAGTACTT-3' \\
\hline Oligo sets human & Forward & Reverse \\
\hline COL2A1 & '5-TGGGTGTTCTATTTATTTATTGTCTTCCT-3' & '5-GCGTTGGACTCACACCAGTTAGT-3' \\
COL10A1 & '5-ATGATGAATACACCAAAGGCTACCT-3' & '5-ACGCACACCTGGTCATTTTCTG-3' \\
RUNX2 & '5-TGATGACACTGCCACCTCTTGA-3' & '5-GCACCTGCCTGGCTCTTCT-3' \\
SOX9 & '5-AGTACCCGCACCTGCACAAC-3' & '5-CGCTTCTCGCTCTCGTTCAG-3' \\
ACAN & '5-GCAGCTGGGCGTTGTCA-3' & '5-TGAGTACAGGAGGCTTGAGGACT-3' \\
NFAT5 & '5-GGGTCAAACGACGAGATTGTG-3' & '5-TTGTCCGTGGTAAGCTGAGAA-3' \\
28S rRNA & '5-GCCATGGTAATCCTGCTCAGTAC-3' & '5-GCTCCTCAGCCAAGCACATAC-3'
\end{tabular}

The 5' to 3' forward and reverse oligonucleotide sequences used for RT-qPCR are listed in the Table.

\subsection{Immunoblotting}

Cells were washed three times with $0.9 \% \mathrm{NaCl}$ and lysed in RIPA buffer $(150 \mathrm{mM} \mathrm{NaCl}$, $1 \%$ NP-40, 0.5\% Sodium deoxycholate, 0.1\% SDS, $50 \mathrm{mM}$ Tris $\mathrm{pH}$ 8.0, $5.0 \mathrm{mM}$ EDTA $\mathrm{pH} 8.0,0.5 \mathrm{mM}$ dithiothreitol and $1 \mathrm{mM}$ phenylmethylsulfonylfluoride). Extracts were sonicated on ice using the Soniprep 150 (MSE, London, UK) at amplitude 10 for 14 cycles (1 second sonication and 1 second pause). Insoluble material was removed by centrifugation $\left(13.000 \mathrm{x} \mathrm{g}, 4^{\circ} \mathrm{C}\right)$. Protein concentration was determined using the $\mathrm{BCA}$ protein assay (Sigma). Polypeptides were separated by SDS-PAGE (samples were equally loaded) and subsequently transferred to nitrocellulose membranes by electroblotting. Primary antibodies for immunodetection were polyclonal goat anti-Col2a1 (Southern Biotech, Birmingham, AL, USA), polyclonal goat anti-Collal (Southern Biotech, Birmingham, AL, USA), polyclonal rabbit anti-Col10a1 (Calbiochem, Darmstadt, Germany), polyclonal rabbit anti-Sox9 (Abcam), mouse monoclonal anti- $\alpha$-Tubulin (Sigma, Saint Louis, MO, USA), polyclonal rabbit anti-Gapdh (Cell Signaling) and polyclonal rabbit anti $-\mathrm{Nfat} 5{ }^{16}$. Bound primary antibodies were detected with rabbit anti-goat, swine anti-rabbit or rabbit anti-mouse immunoglobulins conjugated with horseradish peroxidase (DakoCytomation, Glostrup, Denmark) and visualized by enhanced chemiluminescence 
(ECL). ECL signals were quantified using ImageJ 1.46f software, and relative differences, corrected for background and housekeeper, were determined as compared to control conditions.

\subsection{Cell proliferation}

Cell proliferation was assessed by crystal-violet (Sigma, Saint Louis, MO, USA) staining. Cells were washed two times with $0.9 \% \mathrm{NaCl}$ and subsequently fixed with $4 \%$ paraformaldehyde in phosphate buffered saline for 10 minutes at room temperature. Fixed cells were washed 6 times with water and air dried for storage. Cells were incubated with $0.1 \%$ crystal-violet for 30 minutes at room temperature. Cells were washed six times with water to remove excess crystal-violet and allowed to air-dry in the dark overnight. crystalviolet was extracted from the cells by incubation with $10 \%$ acetic acid for 15 minutes under continuous agitation. Extracted crystal-violet was determined spectrophotometrically at $590 \mathrm{~nm}$ using a plate reader (Biorad, Hemel Hempstead, UK).

\section{$\underline{2.7 \text { Alcian blue staining }}$}

GAG deposition was detected by Alcian blue staining. Cells were washed two times with $0.9 \% \mathrm{NaCl}$ and subsequently fixed with $4 \%$ paraformaldehyde in phosphate buffered saline for 10 minutes at room temperature. Fixed cells were washed 6 times with water and air dried for storage. Fixed cells were incubated overnight with $1 \%$ Alcian blue (Acros Organics, Geel, Belgium) in $0.1 \mathrm{M} \mathrm{HCl}$ at room temperature. Cells were washed six times with water to remove excess Alcian blue and allowed to air-dry in the dark overnight. Alcian blue was extracted from the cells by incubation with Guanidine- $\mathrm{HCl}(6 \mathrm{M})$ for 2 hours under continuous agitation. Extracted Alcian blue was determined spectrophotometrically at $645 \mathrm{~nm}$ using a plate reader (Biorad, Hemel Hempstead, UK).

\section{$\underline{2.8 \text { Statistics }}$}

Statistical significance was determined by two-way ANOVA (with Bonferroni post hoc test) for ATDC5 experiments and two-tailed student t-tests for hBMSC experiments using Graphpad PRISM 5.0 (La Jolla, CA, USA). To test for normal distribution of the input data, D'Agostino-Pearson omnibus normality tests were performed. All quantitative data sets presented passed the normality tests. 


\section{Results}

\subsection{Increased osmolarity improves chondrogenic marker expression in differentiating pro-} genitor cells

To determine whether increased osmolarity improves chondrogenic marker expression in differentiating progenitor cells ${ }^{14}$, the chondroprogenitor cell line ATDC $5^{28,29}$ was differentiated under control conditions and increased osmolarity ( $+100 \mathrm{mOsm}$ and $+200 \mathrm{mOsm})$. Under control conditions ATDC5 cells acquired a chondrogenic phenotype from day 7 in differentiation as determined by increased expression of collagen type II (Col2a1), aggrecan (Acan) and Sox9 (Figure 3.1A). From day 14 in differentiation these cells also express increased levels of the hypertrophic markers collagen type X (Col10a1) and Runx2. Collagen type I (Col1a1) is only slightly induced during ATDC5 differentiation. Protein expression of the above collagens at day 14 in differentiation was confirmed by immunoblotting (Figure 3.1B). Increased osmolarity (+100 mOsm) significantly increased mRNA expression of Sox9 (1.9 fold, p=0.0005), Col2a1 (2 fold, $\mathrm{p}=0.0001)$, Acan (1.4 fold, $\mathrm{p}=0.0018$ ), Runx2 (1.5 fold, $\mathrm{p}=0.0061)$ and Col10a1 (2.5 fold, $\mathrm{p}=0.0001)$ during the course of differentiation when compared to the control condition (Figure 3.1A). Differentiation in +100 mOsm conditions further clearly increased Col2al protein expression. Expression of Col10a1 did not increase as much at protein level as at mRNA level (Figure 3.1B). The decreased Collal mRNA ( $\mathrm{p}=0.0035$ at $\mathrm{t}=14$ days) and protein expression (Figure 3.1B) is in line with our previous findings ${ }^{14}$. Chondrogenic differentiation was inhibited when osmolarity of the differentiation medium was further increased to +200 mOsm, as the expression of all markers was significantly lower when compared to the control condition (at day 14 Sox9: $p=0.0004$, Col2a1: $p=0.0184$, Acan: $p=0.0002$ ), Runx2: $\mathrm{p}=0.0014$ and Col10a1: $\mathrm{p}=0.0004$ ) (Figure 3.1A/B).

Besides mRNA and protein expression of important differentiation markers, glycosaminoglycan (GAG) content is another hallmark of chondrogenic differentiation. At 14 days in differentiation, ATDC5 cells increased total GAG content by 3.2 fold (Figure 3.1C). Similar to mRNA and protein expression of chondrogenic marker genes, $+100 \mathrm{mOsm}$ significantly increased GAG content by another 2.7 fold (control versus +100 mOsm, $\mathrm{p}=0.0018$ ), whereas $+200 \mathrm{mOsm}$ did not increase GAG content compared to $\mathrm{t}=0$. As cell proliferation is another important element in chondrogenic differentiation of $\operatorname{ATDC}^{28}$ and e.g. in cartilage fracture callus development, differences in cell numbers between the con 
A

Sox 9

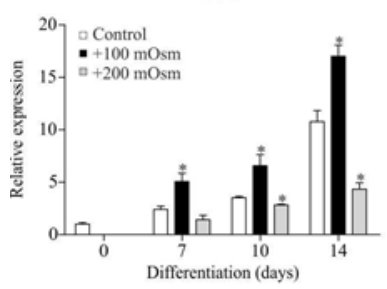

Runx2

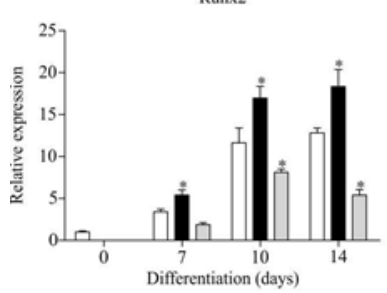

B

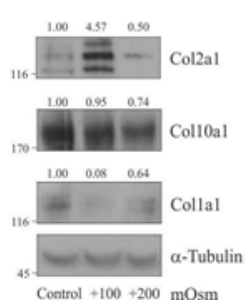

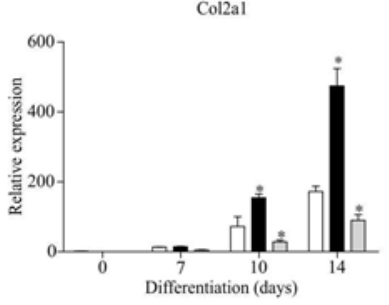

Coll0al

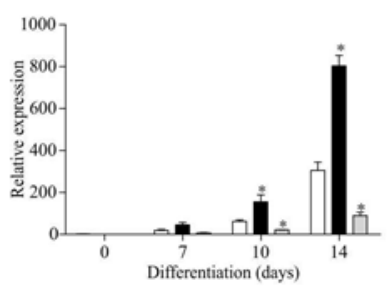

$\mathrm{C}$

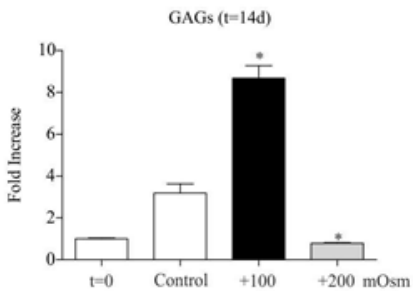

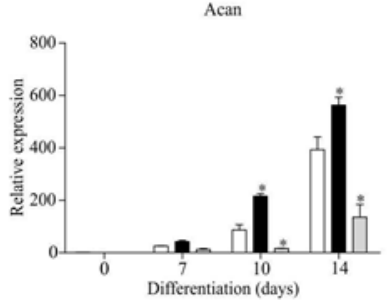

Collal

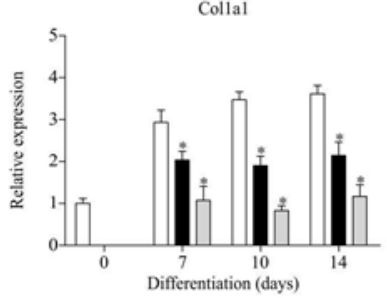

$\mathrm{D}$

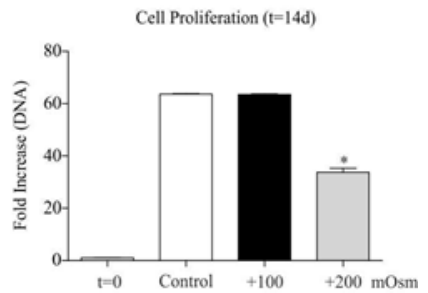

Figure 3.1: Increased osmolarity improves chondrogenic differentiation of ATDC5 cells

ADTC5 cells were differentiated under control osmolarity conditions (control, white bars), +100 mOsm (black bars) and +200 mOsm (grey bars). (A). Induction of Sox9, Col2a1, Aggrecan, Runx2, Col10a1 and Col1a1 mRNA expression was determined by RT-qPCR at day $0,7,10$ and 14 in differentiation. $(B)$. Protein expression of Col2a1, Col10a1 and Collal in day 14 samples, $\alpha$-tubulin was used as loading control. Molecular weight markers $(\mathrm{kDa})$ are depicted on the left of immunoblots. Quantifications of ECL signals (corrected for the complementary $\alpha$-tubulin signals and set relatively to control condition) are depicted on top of all immunoblots. $(C)$. Glycosaminoglycans (GAGs) were stained by Alcian Blue and fold change of $\mathrm{t}=14$ samples was calculated as compared to $\mathrm{t}=0$ samples. $(D)$. Differences in proliferation speed between conditions were determined by crystalviolet staining. Fold change (DNA) from samples from $\mathrm{t}=14$ was calculated relatively to day 0 . In graphs, error bars represent mean \pm s.e.m.. $*$ indicates $p<0.05$, and is determined for control compared to +100 or +200 mOsm. 
ditions were determined. Increasing the osmolarity with 100 mOsm did not influence the increasing cell numbers during differentiation (Figure 3.1D), while culturing under +200 mOsm conditions lead to significantly lower cell numbers (reduction by $\pm 50 \%, p=0.0001$ ) compared to the control condition. Overall, increasing osmolarity with $100 \mathrm{mOsm}$ resulted in an enhanced chondrogenic marker expression during differentiation of ATDC5 cells, while further increasing the osmolarity with another $100 \mathrm{mOsm}$ inhibited overall ATDC5 differentiation.

To further substantiate the pro-chondrogenic effect of increased osmolarity during chondrogenic differentiation of progenitor cells, we differentiated human bone marrow derived mesenchymal stem cells (hBMSCs) from three individuals towards the chondrogenic lineage in control medium and +100 mOsm conditions. As described earlier ${ }^{26}$, chondrogenic/hypertrophic marker expression in these cells is detected around day 21 in differentiation. Increased osmolarity $(+100 \mathrm{mOsm})$ resulted in increased COL2A1 $(\mathrm{p}=0.0005), \operatorname{ACAN}(\mathrm{p}=0.0012)$ and COL10A1 $(\mathrm{p}=0.0001)$ mRNA and protein expression when compared to control conditions (Figure 3.2A/B). Gene expression of their respective transcription factors SOX9 and RUNX2 was also increased in +100 mOsm conditions: SOX9 1.5 fold $(p=0.0449)$ and RUNX2 2.1 fold higher $(p=0.0010)$ (Figure 3.2A). COL1A1 mRNA expression at day 21 in differentiation was only marginally induced in the control condition, but was found to be suppressed under increased osmolarity $(\mathrm{p}=0.0021)$ (Figure 3.2A). Decreased COL1A1 protein expression was also observed under increased osmolarity conditions (Figure 3.2B). Therefore, hBMSC responded similarly to increased osmolarity as ATDC5 cells.

Taken together, we found that the magnitude of chondrogenic differentiation of chondroprogenitor cells is sensitive for the osmolarity of the culture environment, providing the overall indication that chondrogenic marker expression by these cells benefits from osmolarity values in the range of that of articular cartilage.

\subsection{Nfat5 is involved in gene expression under normal and increased osmolarity}

Nfat5/TonEBP is a key regulator of the cellular response to hypertonic conditions and its expression increases during human articular chondrocyte expansion culture in $380 \mathrm{mOsm}$ conditions $^{14}$. We determined the expression of Nfat5 during chondrogenic differentiation of ATDC5 cells under control and +100 mOsm conditions. Nfat5 mRNA expression was found to be upregulated during normal differentiation under control conditions (3.4 fold at day 7 and 5 fold at day 10 and day 14, respectively) (Figure 3.3A). When differentiated in 
A
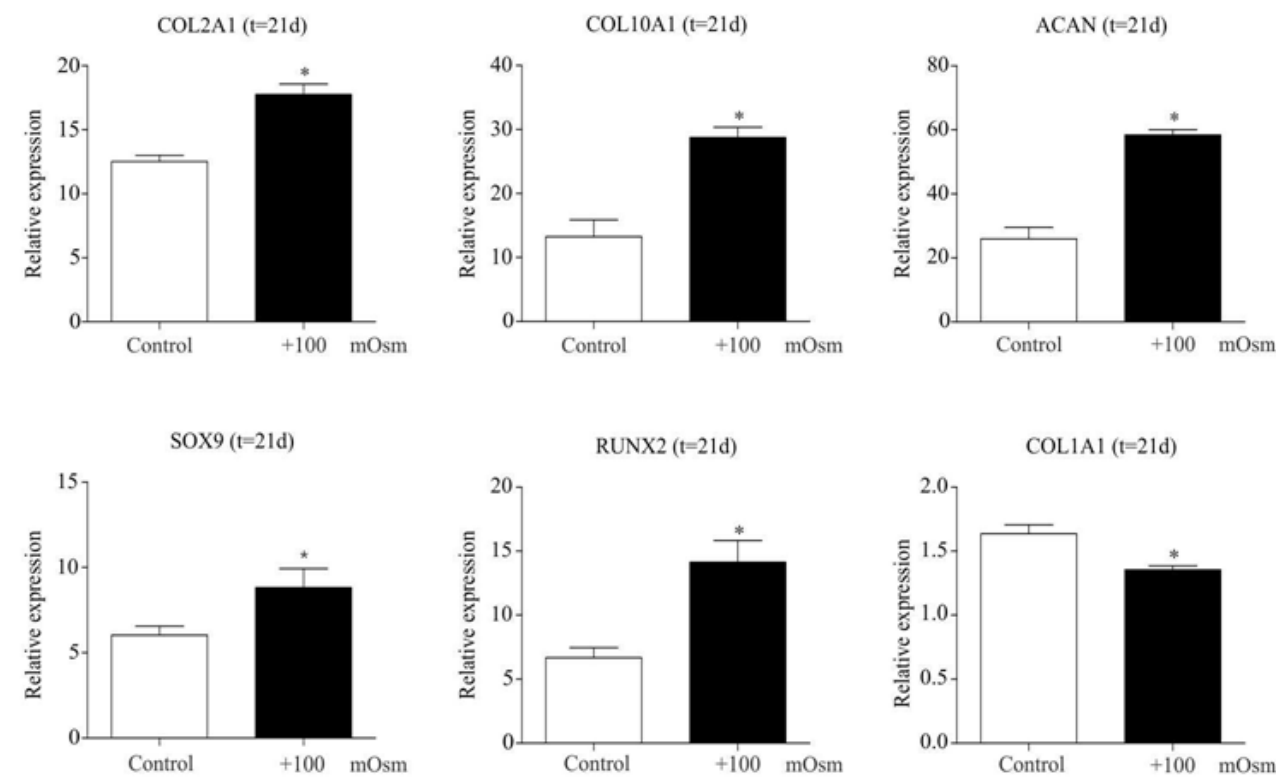

B
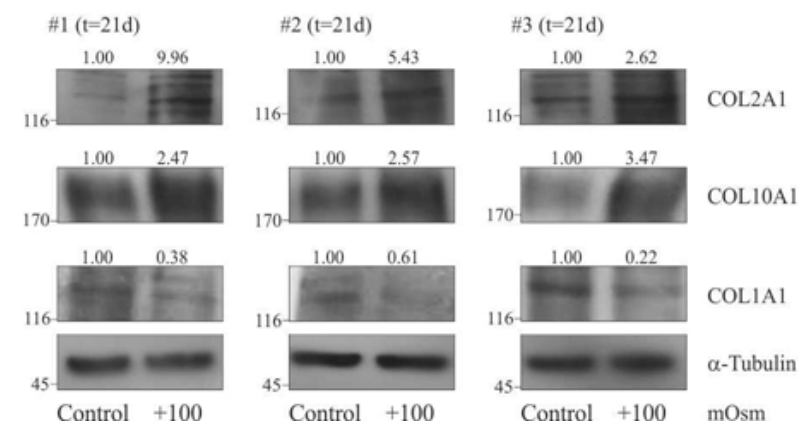

Figure 3.2: Increased osmolarity improves chondrogenic differentiation of hBMSCs

hBMSCs from 3 individual donors were differentiated in the chondrogenic lineage under increased osmolarity. (A). Induction of SOX9, RUNX2, COL2A1, COL10A1, ACAN and COL1A1 mRNA expression was determined by RT-qPCR at day 0 and 21 in differentiation (control, white bars and +100 mOsm black bars). (B). Protein expression of COL2A1, COL10A1 and COL1A1 in day 21 samples. $\alpha$-Tubulin was used as loading control. Molecular weight markers $(\mathrm{kDa})$ are depicted on the left of the immunoblots.Quantifications of ECL signals (corrected for the complementary $\alpha$-tubulin signals and set relatively to control condition) are depicted on top of all immunoblots. In graphs, bars represent the average value of 3 individually averaged values of 3 individual samples per hBMSC isolate and error bars represent mean \pm s.e.m.. * indicates $p<0.05$, and is determined for control versus $+100 \mathrm{mOsm}$. 
+100 mOsm conditions, the expression of Nfat5 was significantly higher as compared to the control condition $(\mathrm{p}=0.0445$ for $\mathrm{t}=7, \mathrm{p}=0.0474$ for $\mathrm{t}=10$ and $\mathrm{p}=0.0195$ for $\mathrm{t}=14$ days) (Figure 3.3A), indicating the osmolarity-responsiveness of Nfat5 also during chondrogenic differentiation. Similarly, NFAT5 mRNA expression in hBMSCs was significantly increased after 21 days of chondrogenic differentiation and also further enhanced under +100 mOsm conditions at day 21 in differentiation $(\mathrm{p}=0.0100)$ as compared to control conditions (Figure 3.3B). Confirming Nfat5 activation upon hyperosmolarity in ATDC5, the expression of the established Nfat5 target genes Slc5a3 and S100a4 specifically increased in +100 mOsm cultures as compared to control cultures $(\mathrm{p}=0.0360$ for Slc5a3 at $\mathrm{t}=14$ days and $\mathrm{p}=0.0175$ for $\mathrm{S} 100 \mathrm{a} 4$ at day 14 ) (Figure $3.3 \mathrm{C})^{14}$.

A

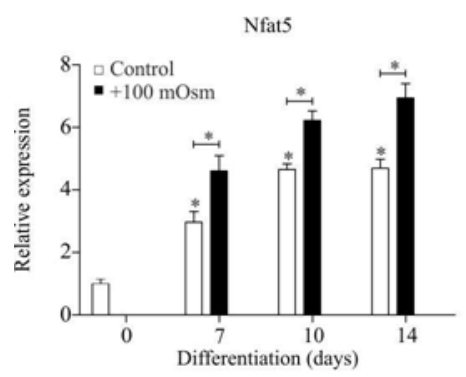

$\mathrm{C}$

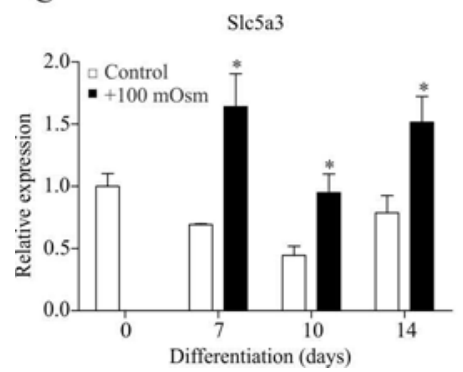

B
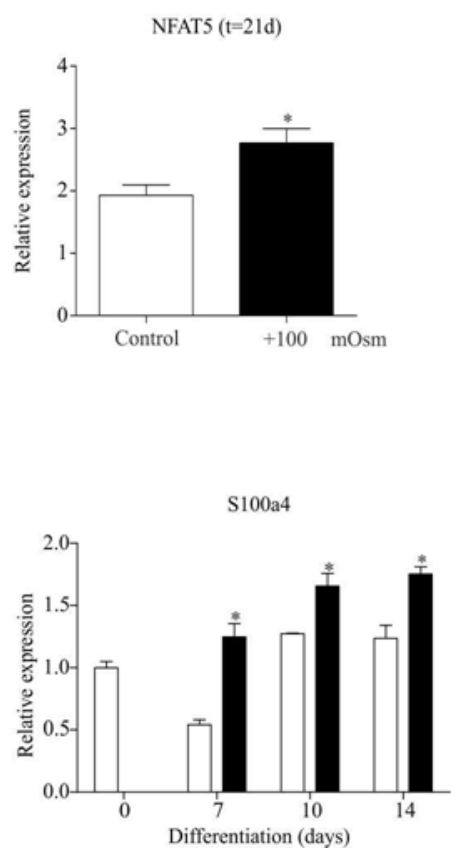

Figure 3.3: Nfat5 expression during chondrogenic differentiation

(A). In similar ATDC5 samples from Figure 3.1A, Nfat5 mRNA expression was determined under control (white bars) and +100 mOsm (black bars) conditions. (B). In similar hBMSC samples from Figure 3.2A, NFAT5 mRNA expression was determined under control (white bars) and +100 mOsm (black bars) conditions. In graphs, bars represent the average value of 3 individually averaged values of 3 individual samples per hBMSC isolate and error bars represent mean \pm s.e.m.. (C). In samples from (A) expression of established Nfat5 target genes (Slc5a3 and S100a4) was determined. In graphs, error bars represent mean \pm s.e.m.* indicates $p<0.05$, and is determined for control versus $+100 \mathrm{mOsm}$. 
To functionally study the involvement of Nfat5 in chondrogenic differentiation under control and +100 mOsm conditions, Nfat5 expression was targeted using RNAi. Transfection of a target-specific siRNA duplex $(30 \mathrm{nM})$ resulted in a 50\% knockdown of Nfat5 mRNA expression in both osmolarity conditions at day $0(p=0.0287), 7(p=0.0415$ for control and $\mathrm{p}=0.0252$ for $+100 \mathrm{mOsm})$ and $10(\mathrm{p}=0.0275$ for control and $\mathrm{p}=0.0225$ for $+100 \mathrm{mOsm}$ ) in differentiation (Figure 3.4A; black versus white bars and dark grey versus light grey bars). Functional Nfat5 knockdown under +100 mOsm conditions was confirmed by immunoblotting (Figure 3.4B), as well as prevented the upregulation of Nfat5 target genes Slc5a3 ( $\mathrm{p}=0.0334$ at day 14) and S100a4 $(\mathrm{p}=0.0091$ at day 14$)$ under increased osmolarity (Figure 3.4C; dark grey versus light grey bars). Knockdown of Nfat5 under control conditions resulted in a significantly suppressed upregulation of chondrogenic markers genes Sox9 (63\%, p=0.0047), Col2a1 (59\%, p=0.0038), Acan (51\%, $\mathrm{p}=0.0033)$, Runx2 $(36 \%, \mathrm{p}=0.0132)$ and Col10a1 (53\%, $\mathrm{p}=0.0350)$ as compared to Mock conditions (scrambled siRNA) during chondrogenic differentiation (Figure 3.4D; black versus white bars and Figure 3.4E). As increased osmolarity not only induced Nfat5 expression (Figure 3.3A/B), but also correlated with increased chondrogenic marker expression (Figure 3.1), we further tested the possibility whether this osmolarity-induced chondrogenic induction may be mediated by Nfat5. Knocking down the expression of Nfat5 under +100 mOsm conditions indeed suppressed the osmolarity effect on chondrogenic gene expression back to normal osmolarity conditions for most of the measured chondrogenic genes (at day 10 Sox9: $p=0.0134$, Col2a1: $p=0.0056$, Acan: $p=0.0065$, Runx2: $\mathrm{p}=0.0324$ and Col10a1: $\mathrm{p}=0.0328$ ) (Figure 3.4D; dark grey versus light grey bars and Figure $3.4 \mathrm{~F}$ ). The osmolarity-induced suppression of Collal mRNA and protein expression was also abrogated by Nfat5 knockdown, where it was expressed even higher than in normal osmolarity conditions (Figure 3.4D; dark grey versus white bars and Figure $3.4 \mathrm{E} / \mathrm{F})$. Together, these findings indicate that Nfat5 is involved in chondrogenic differentiation of ATDC5 cells and that the increased expression of chondrogenic markers during chondrogenic differentiation under +100 mOsm conditions is, at least in part, dependent on Nfat5. 
A

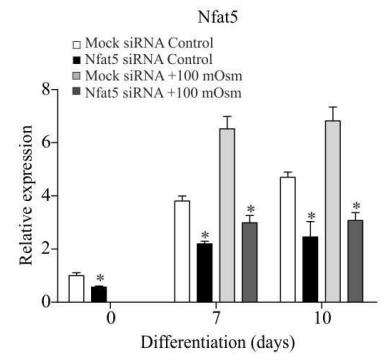

C

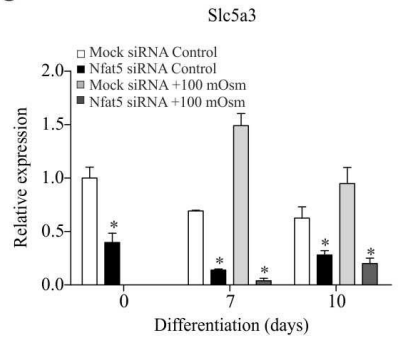

D
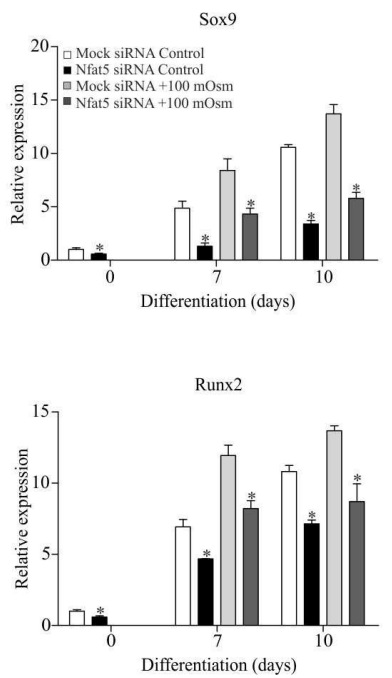

B

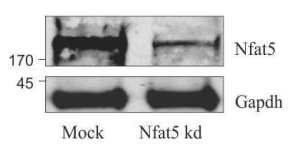

$\mathrm{S} 100 \mathrm{a} 4$

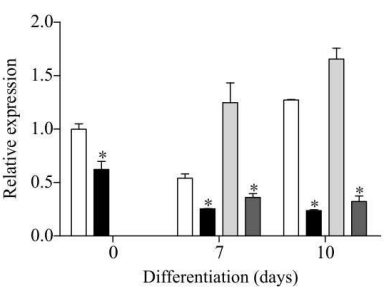

Col2a1

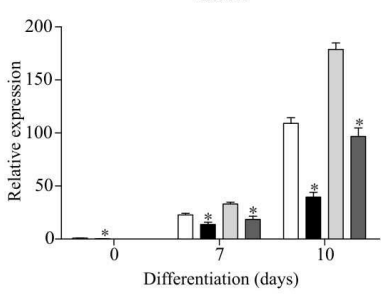

Colloal

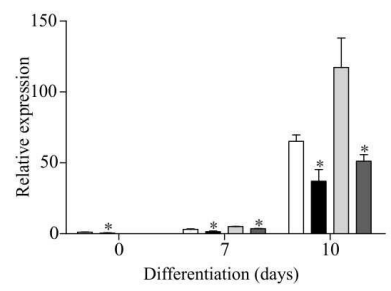

Acan

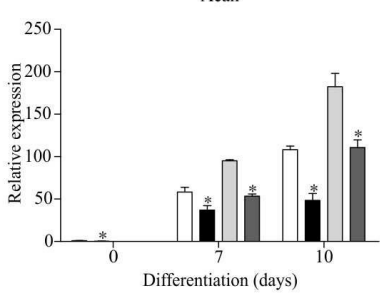

Colla1

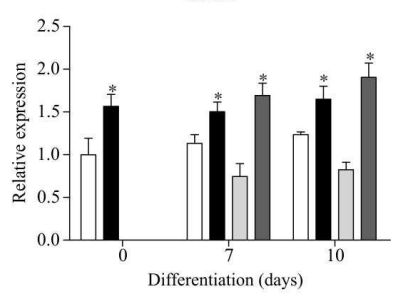


$\mathrm{E}$

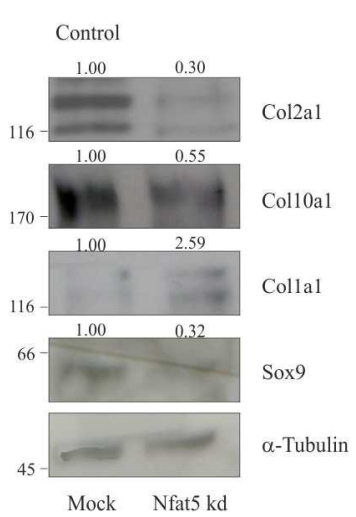

F

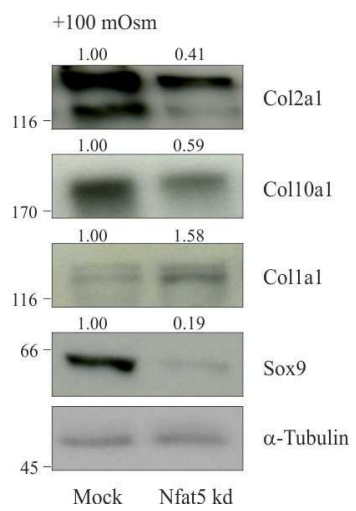

Figure 3.4: Nfat5 may regulate chondrogenic differentiation under normal and increased osmolarity

To determine how Nfat5 is involved in chondrogenic differentiation of ATDC5 cells, expression of Nfat5 was targeted during the course of differentiation by retransfection of an Nfat5 specific siRNA duplex. $(A)$. Knockdown of Nfat5 expression was confirmed at mRNA level at day 0, 7 and 10 in differentiation. White bars represent normal osmolarity conditions (control) transfected with a scrambled siRNA ("Mock") and black bars normal osmolarity conditions transfected with the Nfat5 siRNA. Light grey bars represent increased osmolarity conditions (+100 mOsm) transfected with a scrambled siRNA ("Mock") and black bars increased osmolarity conditions transfected with the Nfat5 siRNA. (B). Knockdown of Nfat5 expression was confirmed by immunoblotting of protein samples from $+100 \mathrm{mOsm}$ conditions at day 10. (C). Knockdown of Nfat5 expression was functionally confirmed by decreased Nfat5 target gene expression of Slc5a3 and S100a4. (D). In similar samples from (A) Sox9, Col2a1, Aggrecan, Runx2, Col10a1 and Col1a1 mRNA expression was determined. (E). Protein expression of Col2a1, Col10a1, Col1a1 and Sox9 from day 10 samples in Mock and Nfat5 knockdown cells under control conditions. (F). Protein expression of Col2a1, Col10a1, Col1a1 and Sox9 from day 10 samples in Mock and Nfat5 knockdown cells under +100 mOsm conditions. In graphs, error bars represent mean \pm s.e.m.. * indicates $\mathrm{p}$ $<0.05$, and is determined for Mock versus Nfat5 siRNA under control osmolarity and under +100 mOsm conditions, respectively.

\subsection{Nfat5 is involved in Sox9 function during chondrogenic differentiation}

To further explore the possibility whether osmolarity induces the increased chondrogenic marker expression via the key chondrogenic transcription factor Sox9 and to explore potential cross-talk between Nfat5 and Sox9, we determined whether osmolarity-driven chondrogenic marker expression can be abolished by interfering with Sox9 expression. Expression of Sox9 was targeted by siRNA transfection ( $30 \mathrm{nM})$ in ATDC5 and resulted in a knockdown efficiency of 58\% at mRNA level at day 0,7 and 10 in differentiation under both control and $+100 \mathrm{mOsm}$ conditions $(\mathrm{p}=0.0398$ at $\mathrm{t}=0, \mathrm{p}=0.0025$ at $\mathrm{t}=10$ control and $\mathrm{p}=0.0123$ at $+100 \mathrm{mOsm}$ ) (Figure $3.5 \mathrm{~A} / \mathrm{C}$; black versus white bars and dark grey versus 
light grey bars). Upon Sox9 knockdown under control conditions, expression of the important Sox9 targets, Col2a1 and aggrecan, was significantly decreased $(p=0.0046$ for Col2a1 and $\mathrm{p}=0.0076$ for Acan at $\mathrm{t}=10$ days) (Figure3.5B; black versus white bars and Figure 3.5C; left panel), showing that expression of these genes under control culture conditions is regulated through Sox9. The osmolarity-induced chondrogenic marker expression under +100 mOsm was also significantly affected by the Sox 9 knockdown $(\mathrm{p}=0.0023$ for $\mathrm{Co} 2 \mathrm{a} 1$ and $\mathrm{p}=0.0092$ for Acan at $\mathrm{t}=10$ days) (Figure 3.5B; dark grey versus light grey bars and Figure 3.5C; right panel), showing that the osmolarity-induced chondrogenic marker expression functionally depends on Sox9.

A

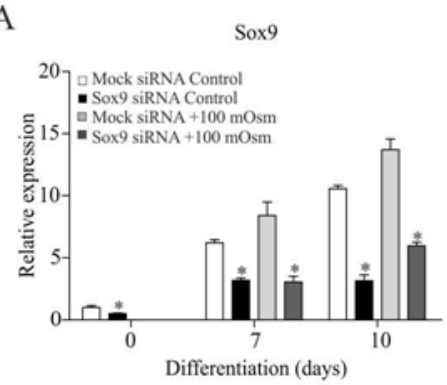

B

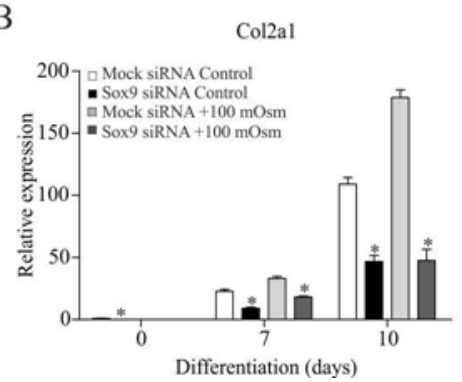

$\mathrm{C}$
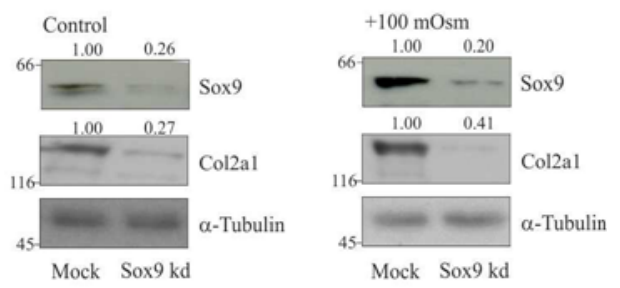

Acan

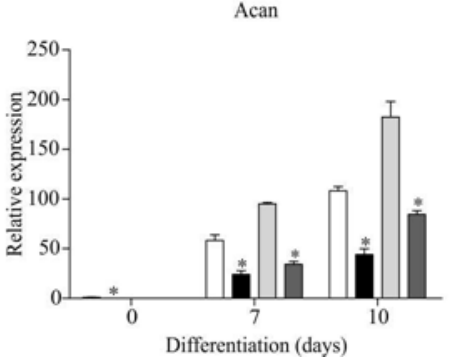

Figure 3.5: Osmolarity-enhanced chondrogenic differentiation is Sox9 dependent

Sox9 siRNA duplexes were retransfected under control and +100 mOsm conditions to investigate whether the osmolarity effect on chondrogenic differentiation is depending on Sox9. (A). Knockdown of Sox9 was confirmed at mRNA level at day 0, 7 and 10 in differentiation. White bars represent normal osmolarity conditions (control) transfected with a scrambled siRNA ("Mock") and black bars normal osmolarity conditions transfected with the Sox9 siRNA. Light grey bars represent increased osmolarity conditions (+100 mOsm) transfected with a scrambled siRNA ("Mock") and black bars increased osmolarity conditions transfected with the Sox9 siRNA. (B). The effect of the Sox 9 knockdown under control and +100 mOsm conditions on chondrogenic differentiation was established by measuring Col2a1 and Acan mRNA expression. $(C)$. Protein expression of Sox9 and Col2a1 from day 10 samples in Mock and Sox 9 knockdown cells under control and +100 mOsm. In graphs, error bars represent mean \pm s.e.m.. * indicates $p<0.05$, and is determined for Mock versus Sox9 siRNA under control osmolarity conditions and under +100 mOsm conditions, respectively. 
We next aimed at elucidating potential cross-talk and pathway hierarchy between Nfat5 and Sox9, which might explain how Nfat5 influences chondrogenic differentiation. We analyzed Sox 9 expression early in ATDC5 differentiation ${ }^{26}$ at 24 hours after Nfat5 knockdown and Nfat5 expression upon Sox9 knockdown at the same moment in ATDC5 differentiation as well. Functional interference with Nfat5 expression was confirmed by decreased expression of the Nfat5 targets Slc5a3 and S100a4 (Figure 3.6A; 68\% $(\mathrm{p}=0.0202)$ and $44 \%(\mathrm{p}=0.0293)$, respectively). In addition, knockdown of Nfat5 efficiently decreased Sox9 mRNA ( $\mathrm{p}=0.0404)$ and protein expression (Figure 3.6A/C). In contrast, knockdown of Sox9 solely decreased Sox9 mRNA ( $\mathrm{p}=0.0339)$ and protein expression, without further affecting Nfat5 mRNA levels or those of its target genes (Figure 3.6B/C). These data suggest that Nfat5 may determine the outcome of ATDC5 chondrogenic differentiation by transcriptionally influencing early expression of Sox9.

A

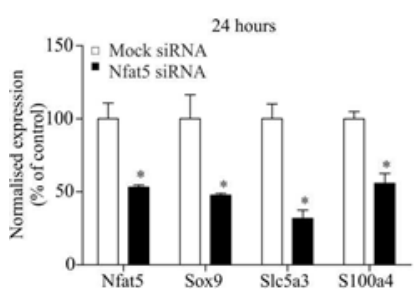

B

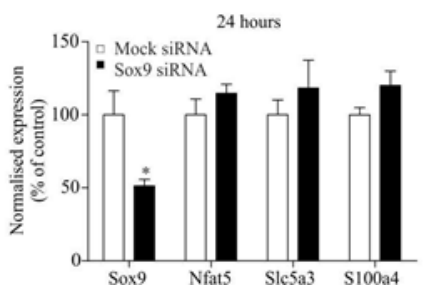

$\mathrm{C}$

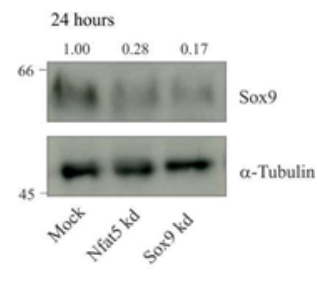

Figure 3.6: Nfat5 influences Sox9 expression

Signalling hierarchy between Nfat5 and Sox9 was determined early (24 hours) in ATDC5 differentiation by siRNA transfections. (A). Expression of Nfat5, Sox9, Slc5a3 and S100a4 mRNAs in differentiating ATDC5 transfected with Mock or Nfat5 siRNAs in control osmolarity conditions. (B). Same as (A) but with Mock or Sox9 siRNAs. Data is presented as percentage to control condition. $(C)$. Sox9 protein expression at 24 hours in ATDC5 differentiation after Mock, Nfat5 or Sox9 siRNA transfection. In graphs, error bars represent mean \pm s.e.m.. * indicates $p<0.05$, and is determined for Mock versus Nfat5 or Sox9 siRNA.

In conclusion, increasing culture medium osmolarity from control levels (i.e. 285-310 mOsm) with 100 mOsm significantly increased chondrogenic marker expression during the course of chondrogenic differentiation of progenitor cells (ATDC5 and hBMSCs). We further found evidence that Nfat5 may play a regulatory role during (osmolarity-induced) chondrogenic differentiation of these cells, at least in part, through influencing Sox9 expression. 


\section{Discussion}

Several studies have shown that human articular chondrocytes in vitro are osmolarityresponsive and increase matrix synthesis under cartilage-physiological osmolarity with Nfat5 being involved in this response. The potentially beneficial effects of increased osmolarity on chondrogenic differentiation of progenitor cells are largely unknown. In addition, the expression and function of Nfat5 during this process have never been described. We here report that chondrogenic marker expression and chondrogenic extracellular matrix synthesis during differentiation of the chondroprogenitor cell line ATDC5 and in human bone marrow stem cells (both sequentially expressing a chondrocyte and hypertrophic chondrocyte phenotype, respectively) positively respond to increased osmolarity. Expression of Nfat5 is upregulated during chondrogenic differentiation and its expression is further upregulated under increased osmolarity. Nfat5 is involved in regulating chondrogenic marker expression under normal and increased osmolarity and may regulate the osmolarity-induced beneficial effects on Col2al and Acan through keychondrogenic transcription factor Sox9.

In human articular cartilage the extracellular osmolarity ranges between 350-480 mOsm $^{9,10}$, due to the high fixed negative charge density of the sulfated GAG side chains in the proteoglycan network. It may therefore not be surprising that chondrocyte phenotypic preservation during isolation and culturing of articular chondrocytes in vitro benefits from increased osmolarity in the culture medium ${ }^{14}$. Following up on these results we determined whether improved expression of chondrogenic marker genes under increased osmolarity could also be found when fully dedifferentiated human articular chondrocytes (HACs) are redifferentiated under increased osmolarity. In agreement with our previous findings ${ }^{12}$ and the data described above, COL2A1 and COL10A1 protein expression, as well as GAG content were increased in the +100 mOsm condition when compared to control condition (Figure 3.7A and B). Similar to above findings, increased osmolarity had no effect on the proliferation capacity of these redifferentiating cells (Figure 3.7C).

However, the osmolarity levels in the direct environment of chondroprogenitor cells in vivo are poorly investigated. Amongst others, mesenchymal stem cells are found in the bone marrow, adipose tissue, muscle, blood and periosteum ${ }^{30}$ and most of these tissues have a normal plasma level osmolarity of around $280 \mathrm{mOsm}$. It is therefore likely that progenitor cells residing in these tissues experience an environment that is close to plasma 
A

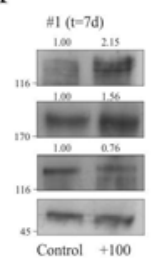

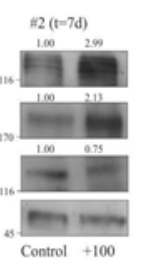

B

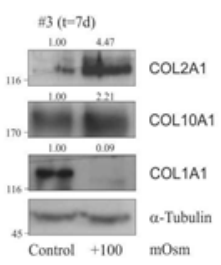

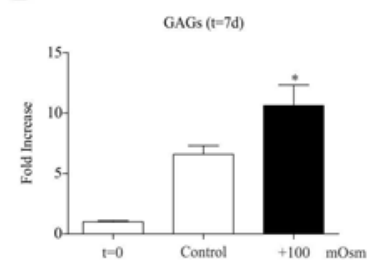

$\mathrm{C}$

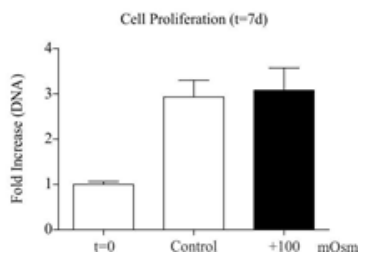

Figure 3.7: Increased osmolarity improves chondrocyte re-differentiation

HACs from 3 individual donors were redifferentiated in the chondrogenic lineage under control and increased osmolarity (+100 mOsm). (A). Protein expression of COL2A1, COL10A1 and COL1A1 from day7 samples was determined by immunoblotting, $\alpha$-tubulin was used as housekeeper. Molecular weight markers (kDa) are depicted on the left. Quantifications of ECL signals (corrected for the complementary $\alpha$-tubulin signals and set relatively to control condition) are depicted on top of all immunoblots. (B). Proteoglycans were stained by alcian blue and fold increase of $\mathrm{t}=7$ samples was calculated from $\mathrm{t}=0$ samples. $(C)$. Differences in proliferation speed between conditions were determined by crystal-violet staining. Fold increase (DNA) from samples from $\mathrm{t}=7$ was calculated relatively to day 0 . In graphs, bars represent the average value of 3 individually averaged values of 3 individual samples per HAC isolate and error bars represent mean \pm s.e.m.. * indicates $p<0.05$.

osmolarity in vivo. Yet, progenitor cells express similar chondrogenic markers (e.g. Sox9, Col2a1, Acan etc.) during chondrogenic differentiation as found in mature articular chondrocytes and may create a local self-sustaining hypertonic microenvironment by excretion of ECM components during their differentiation process. It seems therefore likely that the response of mesenchymal progenitor cells to increased osmolarity may be regulated through similar molecular mechanisms that are also responsible for increased chondrogenic marker expression in mature chondrocytes.

We used established culture protocols for ATDC5 and hBMSC differentiation and thus their corresponding differentiation media had slightly different baseline osmolarities (310 and $285 \mathrm{mOsm}$, respectively), within the range of plasma level osmolarity ${ }^{11,12}$. The relative cell type-specific quantity of the osmotic challenge was, however, identical for both cell types. Noteworthy, for ATDC5 we found that, as opposed to $+100 \mathrm{mOsm}$, an osmotic challenge of $+200 \mathrm{mOsm}$ had deleterious consequences for the chondrogenic differentiation process. This suggests the existence of an optimal osmolarity-window for the outcome of ATDC5 differentiation. The +100 mOsm condition (i.e. $\sim 410 \mathrm{mOsm}$ ) is within the range of physiological articular cartilage osmolarity ${ }^{9,10}$. Higher osmotic pressures, as in the +200 mOsm condition, are rather high end physiological and may exceed membrane transport capacities. 
The molecular mechanism by which osmolarity is sensed by mammalian cells is only partially understood. Nfat5 is accepted as key transcription factor participating in the response to the deleterious effects of increased osmolarity ${ }^{21,31}$. The hypertonic stressinduced increase in intracellular ionic strength is potentially genotoxic, inducing doublestrand DNA breaks, growth arrest and apoptosis ${ }^{32,33}$. Nfat5 is activated by hypertonic stress to compensate for these deleterious effects through activation of compensatory mechanisms like e.g. activating osmolyte transporter gene transcription (e.g. Slc5a3 and S100a4) in order to reduce the intracellular ionic strengths ${ }^{21,34}$. However, little is known about the osmolarity threshold required to activate Nfat5 in specific cell types and several observations suggest that Nfat5 is active already at plasma level osmolarity in tissues that normally are not exposed to a hypertonic environment ${ }^{16}$. In addition, in our experiments knockdown of Nfat5 under plasma level osmolarity resulted in downregulation of Nfat5 target gene expression (Figure 3.4C), indicating that Nfat5 is functionally active under these conditions in the tested progenitor cells. Also, it is becoming increasingly evident that Nfat5 is involved in cellular processes unrelated to hypertonic stress, such as cell proliferation, differentiation or integrin-mediated cell migration ${ }^{31,35,36}$. Our present study shows that Nfat5 expression follows the course of normal chondrogenic differentiation and may play a regulating role during chondrogenic differentiation of progenitor cells. While osmolarity may enhance chondrogenic differentiation through Nfat5, its knockdown during chondrogenic differentiation under both plasma (control) osmolarity and increased osmolarity $(+100 \mathrm{mOsm})$ resulted in suppressed expression of chondrogenic markers Col2a1, Acan, Sox9, Col10a1 and Runx2 (Figure 3.4D-F), suggesting a regulatory function of Nfat5 during chondrogenic differentiation under both control culture conditions and increased osmolarity conditions.

The mode of action by which Nfat5 may regulate chondrogenic differentiation of progenitor cells remains to be elucidated. Our data suggest that Nfat5 might act in this context through influencing the expression of key chondrogenic transcription factor Sox9. Regulation of Sox9 expression by osmolarity has been shown before. Stimulation of human chondrocytes with supraphysiological osmolarity not only induced SOX9 mRNA levels, but also increased SOX9 mRNA half-life ${ }^{37}$. Posttranscriptional stabilization of SOX9 mRNA is activated by p38 MAPK signalling ${ }^{38}$, which is also known to be involved in the hypertonic activation and nuclear translocation of NFAT5 $5^{39-41}$. Alternatively, we previously found that Sox9 expression during chondrogenic differentiation of progenitor cells in vitro is bi-phasic, with a first induction during the first hours of differentiation and 
a second peak expression later on in differentiation. Next to the well documented crucial role of Sox9 during ECM synthesis (late expression peak), we showed that the early and transient induction of Sox 9 crucially determines the chondrogenic outcome of mesenchymal progenitor cell differentiation ${ }^{26}$. Knockdown of Nfat5 resulted in decreased Sox 9 expression (Figure 3.4D) on the long term, which could explain the decreased Col2a1 and Acan expression seen in these cultures. In addition, and relevant to the early Sox 9 induction, Nfat5 knockdown during early chondrogenic differentiation (24 hours) affected early Sox9 expression, as well as known Nfat5 target genes. Reciprocally, knockdown of early Sox9 expression did not influence the expression of Nfat5 or its target genes (Figure 3.6B/ C), suggesting that Nfat5 may act upstream of Sox 9 , through yet unknown (transcriptional) mechanisms.

We have here shown that the magnitude of chondrogenic gene expression of differentiating chondroprogenitor cells in vitro can be enhanced by increasing the osmolarity levels of the culture medium. The osmolarity responsive gene Nfat5 seems to be part of the mechanism that underlies this effect and might influence chondrogenic differentiation via controlling the expression of key chondrogenic transcription factor Sox9. Future studies will focus on further elucidating the relation between $\mathrm{Nfat} 5$ and chondrogenic regulators. Increasing the osmolarity may be used as a relatively simple tool to modulate the formation of cartilaginous tissues for progenitor cell-based cartilage and endochondral bone regenerative medicine approaches ${ }^{42}$.

\section{Acknouledgements}

The authors thank the Dutch Arthritis Association (grant LLP14) and the Dutch Stichting Annafonds|NOREF (grants 07/07 and 08/42) for their financial support and Ing. A. Cremers and Ing. D. Surtel for their technical assistance. Dr. H. Moo Kwon (Department of Medicine, University of Maryland, Baltimore, Maryland, USA) is gratefully acknowledged for providing the TonEBP-specific antibody. 


\section{References}

1 Kronenberg, H. M. Developmental regulation of the growth plate. Nature 423, 332-336 (2003).

2 Mackie, E. J., Ahmed, Y. A., Tatarczuch, L., Chen, K. S. \& Mirams, M. Endochondral ossification: how cartilage is converted into bone in the developing skeleton. Int J Biochem Cell Biol 40, 46-62 (2008).

3 Zuscik, M. J., Hilton, M. J., Zhang, X., Chen, D. \& O'Keefe, R. J. Regulation of chondrogenesis and chondrocyte differentiation by stress. J Clin Invest 118, 429438 (2008).

4 de Crombrugghe, B., Lefebvre, V. \& Nakashima, K. Regulatory mechanisms in the pathways of cartilage and bone formation. Curr Opin Cell Biol 13, 721-727 (2001).

5 Lefebvre, V. \& Smits, P. Transcriptional control of chondrocyte fate and differentiation. Birth Defects Res C Embryo Today 75, 200-212, doi:10.1002/bdrc.20048 (2005).

6 Mow, V. C. \& Atheshian, G. A. in Basic Orthopaedic Biomechanics (eds V.C. Mow \& W.C. Hayes) 275-315 (Lippincott-Raven, Philidelphia (PA), 1997).

7 Lesperance, L. M., Gray, M. L. \& Burstein, D. Determination of fixed charge density in cartilage using nuclear magnetic resonance. J Orthop Res 10, 1-13, doi:10.1002/jor.1100100102 (1992).

8 Venn, M. \& Maroudas, A. Chemical composition and swelling of normal and osteoarthrotic femoral head cartilage. I. Chemical composition. Annals of the rheumatic diseases 36, 121-129 (1977).

9 Urban, J. P. The chondrocyte: a cell under pressure. Br J Rheumatol 33, 901-908 (1994).

10 Urban, J. P., Hall, A. C. \& Gehl, K. A. Regulation of matrix synthesis rates by the ionic and osmotic environment of articular chondrocytes. J Cell Physiol 154, 262270, doi:10.1002/jcp.1041540208 (1993).

11 Bhalla, A. et al. Influence of raised plasma osmolality on clinical outcome after acute stroke. Stroke 31, 2043-2048 (2000).

12 Charkoudian, N., Eisenach, J. H., Joyner, M. J., Roberts, S. K. \& Wick, D. E. Interactions of plasma osmolality with arterial and central venous pressures in control of sympathetic activity and heart rate in humans. Am J Physiol Heart Circ Physiol 289, H2456-2460, doi:10.1152/ajpheart.00601.2005 (2005).

13 Palmer, G. D. et al. Time-dependent aggrecan gene expression of articular chondrocytes in response to hyperosmotic loading. Osteoarthritis Cartilage 9, 761770, doi:10.1053/joca.2001.0473 S106345840190473X [pii] (2001).

14 van der Windt, A. E. et al. Physiological tonicity improves human chondrogenic marker expression through nuclear factor of activated T-cells 5 in vitro. Arthritis research \& therapy 12, R100, doi:10.1186/ar3031 (2010).

15 van der Windt, A. E. et al. Inhibiting calcineurin activity under physiologic tonicity elevates anabolic but suppresses catabolic chondrocyte markers. Arthritis and rheumatism 64, 1929-1939, doi:10.1002/art.34369 (2012).

16 Miyakawa, H., Woo, S. K., Dahl, S. C., Handler, J. S. \& Kwon, H. M. Tonicityresponsive enhancer binding protein, a rel-like protein that stimulates transcription in response to hypertonicity. Proc Natl Acad Sci U S A 96, 2538-2542 
(1999).

17 Lopez-Rodriguez, C. et al. Loss of NFAT5 results in renal atrophy and lack of tonicity-responsive gene expression. Proc Natl Acad Sci U S A 101, 2392-2397, doi:101/8/2392 [pii] (2004).

18 Na, K. Y., Woo, S. K., Lee, S. D. \& Kwon, H. M. Silencing of TonEBP/NFAT5 transcriptional activator by RNA interference. J Am Soc Nephrol 14, 283-288 (2003).

19 Chen, M., Sastry, S. K. \& O'Connor, K. L. Src kinase pathway is involved in NFAT5-mediated S100A4 induction by hyperosmotic stress in colon cancer cells. American journal of physiology. Cell physiology 300, C1155-1163, doi:10.1152/ ajpcell.00407.2010 (2011).

20 Chen, M., Sinha, M., Luxon, B. A., Bresnick, A. R. \& O'Connor, K. L. Integrin alpha6beta4 controls the expression of genes associated with cell motility, invasion, and metastasis, including S100A4/metastasin. J Biol Chem 284, 1484-1494, doi:M803997200 [pii] 10.1074/jbc.M803997200 (2009).

21 Woo, S. K., Lee, S. D. \& Kwon, H. M. TonEBP transcriptional activator in the cellular response to increased osmolality. Pflugers Arch 444, 579-585, doi:10.1007/s00424-002-0849-2 (2002).

22 Halterman, J. A., Kwon, H. M. \& Wamhoff, B. R. Tonicity-independent regulation of the osmosensitive transcription factor TonEBP (NFAT5). American journal of physiology. Cell physiology 302, C1-8, doi:10.1152/ajpcell.00327.2011 (2012).

23 Yoon, H. J. et al. NFAT5 is a critical regulator of inflammatory arthritis. Arthritis and rheumatism 63, 1843-1852, doi:10.1002/art.30229 (2011).

24 Jauliac, S. et al. The role of NFAT transcription factors in integrin-mediated carcinoma invasion. Nat Cell Biol 4, 540-544, doi:10.1038/ncb816 (2002).

25 Schallmoser, K. et al. Rapid large-scale expansion of functional mesenchymal stem cells from unmanipulated bone marrow without animal serum. Tissue engineering 14, 185-196 (2008).

26 Caron, M. M. et al. Activation of NF-kappaB/p65 Facilitates Early Chondrogenic Differentiation during Endochondral Ossification. PLoS One 7, e33467, doi:10.1371/journal.pone.0033467 (2012).

27 Welting, T. J. et al. Inhibition of cyclooxygenase-2 impacts chondrocyte hypertrophic differentiation during endochondral ossification. European cells \& materials 22, 420-436; discussion 436-427 (2011).

28 Atsumi, T., Miwa, Y., Kimata, K. \& Ikawa, Y. A chondrogenic cell line derived from a differentiating culture of AT805 teratocarcinoma cells. Cell Differ Dev 30, 109-116 (1990).

29 Chen, L., Fink, T., Zhang, X. Y., Ebbesen, P. \& Zachar, V. Quantitative transcriptional profiling of ATDC5 mouse progenitor cells during chondrogenesis. Differentiation 73, 350-363 (2005).

30 Barry, F. P. \& Murphy, J. M. Mesenchymal stem cells: clinical applications and biological characterization. Int J Biochem Cell Biol 36, 568-584, doi:10.1016/ j.biocel.2003.11.001 (2004).

31 Aramburu, J. et al. Regulation of the hypertonic stress response and other cellular functions by the Rel-like transcription factor NFAT5. Biochem Pharmacol 72, 1597-1604, doi:10.1016/j.bcp.2006.07.002 (2006).

32 Christoph, K., Beck, F. X. \& Neuhofer, W. Osmoadaptation of Mammalian cells an orchestrated network of protective genes. Curr Genomics 8, 209-218 (2007).

33 Racz, B. et al. Hyperosmotic stress-induced apoptotic signaling pathways in 
chondrocytes. Bone 40, 1536-1543, doi:S8756-3282(07)00065-8 [pii] 10.1016/j.bone.2007.02.011 (2007).

34 Ho, S. N. The role of NFAT5/TonEBP in establishing an optimal intracellular environment. Arch Biochem Biophys 413, 151-157 (2003).

35 Maouyo, D. et al. Mouse TonEBP-NFAT5: expression in early development and alternative splicing. American journal of physiology. Renal physiology 282, F802809, doi:10.1152/ajprenal.00123.2001 (2002).

36 O'Connor, R. S., Mills, S. T., Jones, K. A., Ho, S. N. \& Pavlath, G. K. A combinatorial role for NFAT5 in both myoblast migration and differentiation during skeletal muscle myogenesis. Journal of cell science 120, 149-159, doi:10.1242/ jes.03307 (2007).

37 Tew, S. et al. Hyperosmolarity regulates SOX9 mRNA post transcriptionally in human articular chondrocytes. American journal of physiology 297, C898 - C906, doi:00571.2008 [pii] 10.1152/ajpcell.00571.2008 (2009).

38 Tew, S. R. \& Hardingham, T. E. Regulation of SOX9 mRNA in human articular chondrocytes involving p38 MAPK activation and mRNA stabilization. The Journal of biological chemistry 281, 39471-39479, doi:10.1074/jbc.M604322200 (2006).

39 Ko, B. C. et al. Fyn and p38 signaling are both required for maximal hypertonic activation of the osmotic response element-binding protein/tonicity-responsive enhancer-binding protein (OREBP/TonEBP). The Journal of biological chemistry 277, 46085-46092, doi:10.1074/jbc.M208138200 (2002).

40 Lee, J. H. et al. NFAT5 induction and its role in hyperosmolar stressed human limbal epithelial cells. Invest Ophthalmol Vis Sci 49, 1827-1835, doi:10.1167/ iovs.07-1142 (2008).

41 Morancho, B., Minguillon, J., Molkentin, J. D., Lopez-Rodriguez, C. \& Arambu$\mathrm{ru}$, J. Analysis of the transcriptional activity of endogenous NFAT5 in primary cells using transgenic NFAT-luciferase reporter mice. BMC $\mathrm{Mol}$ Biol 9, 13, doi:10.1186/1471-2199-9-13 (2008).

42 Emans, P. J. et al. Autologous engineering of cartilage. Proc Natl Acad Sci U S A 107, 3418-3423, doi:0907774107 [pii]10.1073/pnas.0907774107 (2010). 



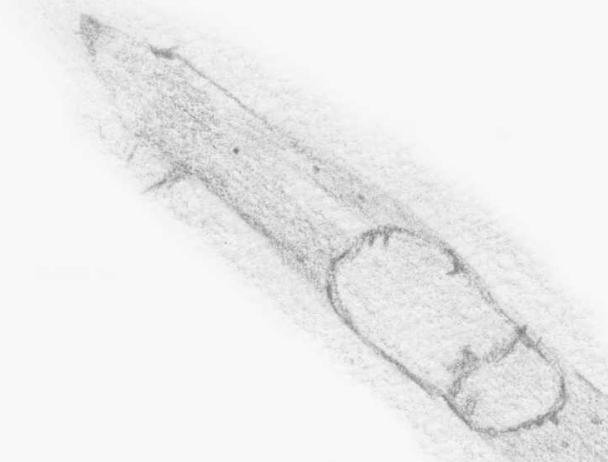

Chapter 4

\section{Hypertrophic Differentiation During Chondrogenic Differentiation}

of Progenitor Cells is Stimulated by Bone Morphogenic Protein-2

but Suppressed by Bone Morphogenic Orotein-7

Adapted from:

Hypertrophic Differentiation During Chondrogenic Differentiation of Progenitor cells is stimulated by BMP-2 but suppressed by BMP-7

Marjolein M.J. Caron, Pieter J.Emans, Andy Cremers, Don A.M. Surtel, Mariëlle M.E. Coolsen, Lodewijk W. van Rhijn and Tim J. M. Welting

Department of Orthopaedic Surgery, Maastricht University Medical Center, Maastricht, the Netherlands Osteoarthritis and Cartilage 2013; In press 


\section{Alstract}

Introduction: Bone morphogenic protein (BMP)-2 and BMP-7 are clinically approved and their recombinant proteins are used for bone tissue regenerative purposes and widely evaluated for cartilage regeneration. Previous comparison of the in vitro chondrogenic characteristics of BMP-2 versus BMP-7 did not address hypertrophic differentiation and characterizing their chondrogenic properties with a focus in on chondrocyte hypertrophy was topic of investigation in this study.

Methodology: Equimolar concentrations of BMP-2 or BMP-7 were added to chondrogenic differentiating ATDC5, human bone marrow stem cells or rabbit periosteal explants. Expression of Col2a1, Sox9, Acan, Col10a1, Runx2, ALP, Mmp13, Mef2c and Bapx1/ Nkx3.2 was determined by RT-qPCR and immunoblotting. Glycosaminoglycan content, cell proliferation capacity and ALP activity were analysed by colorimetric analyses. Expression of Bapx1/Nkx3.2 and Sox9 was targeted by transfection of target specific siRNA duplexes.

Results: BMP-2 dose-dependently increased chondrocyte hypertrophy during chondrogenic differentiation of progenitor cells, whereas BMP-7 acted hypertrophy suppressive and chondro-promotive. Both BMPs did not influence cell proliferation, but they did increase total glycosaminoglycan content. In a candidate approach Bapx1/Nkx3.2 was found to be involved in the BMP-7 mediated suppression of chondrocyte hypertrophy in ATDC5 cells.

Conclusions: BMP-2 and BMP-7 display opposing actions on the chondrogenic outcome of differentiating progenitor cells: BMP-2 acts a specific inducer of chondrocyte hypertrophy, while BMP-7 appears to increase or maintain chondrogenic potential and prevent chondrocyte hypertrophy. Our results pave the way for an application-dependent differential use of BMP-2 or BMP-7. 


\section{Introduction}

Methodologies controlling chondrogenic differentiation of mesenchymal progenitor cells towards chondrocytes are of interest to improve the outcome of skeletal regenerative medicine approaches. During chondrogenic differentiation mesenchymal progenitor cells condensate and differentiate into proliferating and extracellular matrix synthesizing chondrocytes which, in the case of endochondral ossification and different cartilage pathologies, ultimately terminally differentiate into hypertrophic mineralized chondrocytes $^{1,2}$. For progenitor cell-based cartilage regeneration it is therefore crucial to find optimal strategies to stimulate progenitor cells to differentiate into chondrocytes and preventing them from hypertrophy and mineralization ${ }^{3,4}$. On the contrary, for bone tissue engineering, promoting terminal differentiation through endochondral ossification may be advantageous ${ }^{5}$.

Growth factors are important soluble mediators for tuning a variety of cellular processes including cell proliferation and differentiation. In this respect, members of the transforming growth factor (TGF)- $\beta$ superfamily are frequently used to promote chondrogenic differentiation processes in vitro ${ }^{6,7}$. A separate class of TGF- $\beta$ superfamily-members are the bone morphogenic proteins (BMPs). These proteins were discovered in extracts of demineralized bone and were demonstrated to induce de novo endochondral bone formation in vivo when administered ectopically ${ }^{8}$. Especially BMP-2 and BMP-7 (also known as osteogenic protein-1 (OP-1)) are clinically approved and their recombinant proteins are used to promote healing at sites of poor bone fracture healing (non-unions), as additional factors to enhance integration of bone grafts and are widely evaluated for cartilage and bone tissue regenerative purposes ${ }^{9-11}$. BMPs are involved in all phases of chondrogenic differentiation and are able to directly regulate expression of several chondrocyte specific genes ${ }^{12,13}$. BMP-2 is described to promote cell proliferation and matrix synthesis in human articular chondrocytes, growth plate chondrocytes and developing mouse limbs ${ }^{14-16}$. Moreover, BMP-2 has been demonstrated to regulate the expression and activity of key-chondrogenic transcription factor Sox9 (SRY (sex determining region Y) box9 $)^{17,18}$. BMP-7 is described to promote in vitro chondrogenic extracellular matrix synthesis in normal and osteoarthritic human articular chondrocytes ${ }^{14,19-21}$ and can counteract retinoic acid-induced dedifferentiation of bovine chondrocytes ${ }^{22}$. In addition, BMP-7 also promotes chondrogenic differentiation of human and goat perichondrium 
cells, developing mouse limbs and human chondrocytes ${ }^{23}$.

Although these BMPs are clinically used and their potential is widely explored in cartilage/ bone regenerative approaches, the hypertrophic properties of BMP-2 versus BMP-7 are not well documented. We aimed to compare the action of BMP-2 and BMP-7 on chondrogenic differentiation of progenitor cells, focusing on hypertrophic differentiation, using ATDC5 cells and human bone-marrow-derived mesenchymal stem cells. These both cell models are known to eventually show chondrocyte hypertrophy in vitro. Our results demonstrate that BMP-2 and BMP-7 display opposing effects on the chondrogenic outcome of differentiating progenitor cells: BMP-2 acts a specific inducer of chondrocyte hypertrophy, while BMP-7 appears to increase or maintain chondrogenic potential and suppress chondrocyte hypertrophy. Further focussing of BMP-7 we found that Bapx1/Nkx3.2 (Bagpipe homeobox gene 1 homolog/NK3 homeobox 2) is involved in the hypertrophysuppressive action of this BMP. Together our results may provide a basis for differential use of BMP-2 or BMP-7, depending on a chondrogenic or endochondral/bone application purpose.

\section{Materials and Methods}

\section{$\underline{2.1 \text { ATDC5 cell culture }}$}

ATDC5 were cultured (as described before ${ }^{24}$ ) in proliferation medium consisting of DMEM/F12 (Invitrogen, Carlsbad, CA, USA), 5\% FCS (PAA, Pasching, Austria), 1\% antibiotic/antimycotic (Invitrogen) and 1\% NEAA (non-essential amino acids; Invitrogen). ATDC5 were differentiated in differentiation medium comprising proliferation medium supplemented with $10 \mu \mathrm{g} / \mathrm{ml}$ insulin (Sigma, St. Louis, MO, USA), $10 \mu \mathrm{g} / \mathrm{ml}$ transferrin (Roche Applied Science) and $30 \mathrm{nM}$ sodium selenite (Sigma). Cells were plated at 6400 cells $/ \mathrm{cm}^{2}$ and chondrogenic differentiation was initiated by adding differentiation medium. BMP-2 (Sigma) or BMP-7 (R\&D Systems, Minneapolis, MN, USA) were used in equimolar concentrations. For calculation of equimolar concentrations, disulphide-linked homodimers of both BMPs were regarded as the functional ligands. For comparison, $1 \mathrm{nM}$ BMP-2 corresponds to $31 \mathrm{ng} / \mathrm{ml}$ and $1 \mathrm{nM}$ BMP-7 corresponds to $26 \mathrm{ng} / \mathrm{ml}$ ). For RNAiexperiments a Bapx1/Nkx3.2 siRNA duplex (sense: 5'-CAGAGACGCAAGUGAAGAUT T-3', anti-sense: 5'-AUCUUCACUUGCGUCUCUGTT-3'), Sox9 siRNA duplex (sense: 
5'- GACUCACAUCUCUCCUAAUTT-3', anti-sense: 5'- AUUAGGAGAGAUGUGAGU CTT-3') and scrambled siRNA-duplex (indicated by "Mock") were used (Eurogentec, Liège, Belgium). ATDC5 cells were seeded at 25,000 cells $/ \mathrm{cm}^{2}$ and transfection $(50 \mathrm{nM})$ was performed using ICAfectin 442 (Eurogentec) according to manufacturers' protocol. This resulted in an average transfection efficiency of $75 \%$ in these experiments (determined by fluorescence microscopy of 5' FITC labeled variants of the respective siRNAs). Transfected cells were cultured for 1 day before chondrogenic differentiation was initiated and retransfection of siRNAs was carried out at 2 and 5 days.

\section{$\underline{2.2 \mathrm{hBMSC} \text { isolation and cell culture }}$}

Human bone marrow stem cells (hBMSCs) were obtained from iliac crest bone marrow aspirate from young, genetically healthy individuals (MEC MUMC approval 08-4-056). hBMSCs were isolated using Ficoll-Paque PLUS (Amersham Pharmacia, Diegem, Belgium). Culture medium consisted of DMEM high glucose (Invitrogen), 10\% FCS, 1\% antibiotic/antimycotic and 1\% NEAA. Chondrogenic differentiation was performed in monolayer by plating passage 5 cells at 30,000 cells $/ \mathrm{cm}^{2}$. Differentiation medium consisted of proliferation medium supplemented with $1 \%$ ITS (Invitrogen), $50 \mathrm{mg} / \mathrm{ml}$ L-ascorbic acid-2-phosphate (Sigma) and $1 \mathrm{ng} / \mathrm{ml}$ TGF $\beta 3$ (R\&D) ${ }^{24,25}$. BMP-2 or BMP-7 were used in equimolar concentrations as described above.

\subsection{Periosteum Agarose Culture (PAC) chondrogenic differentiation}

As previously described ${ }^{26}$, periosteum was harvested from the proximal tibia of skeletally mature NZW-rabbits. Post-mortem animals were obtained from an unrelated study, no ethical approval was necessary. Periosteum was prepared as a $3 \times 3 \mathrm{~mm}$ piece and embedded between a layer of high melting agarose (1\%) and low melting agarose (1\%) according to O'Driscoll et al. ${ }^{27}$, and proliferation medium (DMEM/F12, 10\% FCS, 1\% P/ $\mathrm{S}, 1 \%$ NEAA) was added. After overnight incubation, medium was changed to differentiation medium (proliferation medium supplemented with $1 \%$ ITS, $50 \mathrm{mg} / \mathrm{ml} \mathrm{L}$-ascorbic acid2-phosphate, $10 \mathrm{ng} / \mathrm{ml} \mathrm{TGFb3)} \mathrm{and} \mathrm{PACs} \mathrm{were} \mathrm{differentiated} \mathrm{for} 20$ days with medium changes every 3 days. BMP-2 or BMP-7 were used in equimolar concentrations as described above.

\section{$\underline{2.4 R T-q P C R}$}

For RNA isolation, cells were washed 3 times with $0.9 \% \mathrm{NaCl}$ and disrupted with $500 \mu \mathrm{l}$ 
Trizol (Invitrogen). To isolate total RNA from PAC cultures, tissue was cut in pieces and soaked overnight in $500 \mathrm{ml}$ Trizol at $4^{\circ} \mathrm{C}$. RNA isolation, RNA quantification by ultraviolet (UV)-spectrometry (Nanodrop, Thermo Scientific), and cDNA synthesis were performed as described before ${ }^{24}$. Real time quantitative PCR (RT-qPCR) was performed using Mesagreen qPCR mastermix plus for SYBR ${ }^{\circledR}$ Green (Eurogentec). An Applied Biosystems ABI PRISM 7700 Sequence Detection System was used for amplification using the following protocol: initial denaturation at $95^{\circ} \mathrm{C}$ for $10 \mathrm{~min}$, followed by 40 cycles of DNA amplification (denaturing for $15 \mathrm{~s}$ at $95^{\circ} \mathrm{C}$ and annealing for $1 \mathrm{~min}$ at $60^{\circ} \mathrm{C}$ ) followed by a dissociation curve. Data were analysed using the standard curve method, mRNA expression was normalized to a reference gene ( $\beta$-actin (ATDC5, rPACs) and 28S rRNA (hBMSCs)) and gene expression was calculated as fold increase as compared to day 0 . Validated primer sequences are depicted in Table 4.1 .

Table 4.1: DNA oligo sequences for RT-qPCR.

\begin{tabular}{|c|c|c|}
\hline$\underline{\text { Oligo sets mouse }}$ & $\underline{\text { Forward }}$ & $\underline{\text { Reverse }}$ \\
\hline $\mathrm{Col} 2 \mathrm{a} 1$ & '5-TGGGTGTTCTATTTATTTATTGTCTTCC-3’ & '5-GCGTTGGACTCACACCAGTTAGT-3' \\
\hline Runx2 & '5-CGATGAAGACCCCAACCCTAA-3’ & '5-ACTGGTAATGGCATCAAGGGATA-3' \\
\hline Acan & '5-CATGAGAGAGGCGAATGGAA-3 & '5-TGATCTCGTAGCGATCTTTCTTCT-3' \\
\hline $\operatorname{Mef} 2 \mathrm{c}$ & '5-GGGCCTCAATGGCTGTGA-3' & '5-CTCAGACTCAGGGCTGTGACCTA-3' \\
\hline Mmp13 & '5-CGATGAAGACCCCAACCCTAA-3’ & '5-ACTGGTAATGGCATCAAGGGATA-3' \\
\hline Oligo sets human & $\underline{\text { Forward }}$ & $\underline{\text { Reverse }}$ \\
\hline COL $2 \mathrm{~A} 1$ & '5-TGGGTGTTCTATTTATTTATTGTCTTCCT-3' & '5-GCGTTGGACTCACACCAGTTAGT-3' \\
\hline COL10A1 & '5-ATGATGAATACACCAAAGGCTACCT-3’ & '5-ACGCACACCTGGTCATTTTCTG-3' \\
\hline RUNX2 & '5-TGATGACACTGCCACCTCTTGA-3' & '5-GCACCTGCCTGGCTCTTCT-3' \\
\hline 28S rRNA & '5-GCCATGGTAATCCTGCTCAGTAC-3' & '5-GCTCCTCAGCCAAGCACATAC-3' \\
\hline$\underline{\text { Oligo sets rabbit }}$ & Forward & $\underline{\text { Reverse }}$ \\
\hline
\end{tabular}

Forward and reverse oligo sequences ( $\left.5^{\prime}-3^{\prime}\right)$ used for RT-qPCR are listed for mouse, rabbit and human. 


\subsection{Immunoblotting}

Cells were washed with $0.9 \% \mathrm{NaCl}$ and lysed in RIPA buffer. Extracts were sonicated on ice using the Soniprep 150 (MSE) at amplitude 10 for 14 cycles ( 1 second sonication and 1 second pause). Insoluble material was removed by centrifugation $\left(13.000 \mathrm{x} \mathrm{g}, 4^{\circ} \mathrm{C}\right)$. Protein concentration was determined using the bicinchoninic acid (BCA) protein assay (Sigma). Polypeptides were separated by SDS-PAGE (samples were equally loaded) and transferred to nitrocellulose membranes by electroblotting. Primary antibodies for immunodetection were polyclonal goat anti-Col2a1 (Southern Biotech, Birmingham, AL, USA), polyclonal rabbit anti-Col10a1 (Calbiochem, Darmstadt, Germany), polyclonal goat antiSox9 (Abcam, Cambridge, UK), mouse monoclonal anti-Runx2 (MBL, Woburn, MA, USA), polyclonal rabbit anti-Bapx1 (Abcam, Cambridge, UK) and mouse monoclonal anti $-\alpha$-Tubulin (Sigma). Bound primary antibodies were detected with rabbit anti-goat, swine anti-rabbit or rabbit anti-mouse immunoglobulins conjugated with horseradish peroxidase (DakoCytomation, Glostrup, Denmark) and visualized by enhanced chemiluminescence (ECL). ECL signals were quantified using ImageJ 1.46f software. Relative differences, corrected for background and housekeeper, were determined as compared to control conditions.

\section{$\underline{\text { 2.6 Alcian blue staining }}$}

Glycosaminoglycan content was detected by Alcian blue staining. Cells were washed with $0.9 \% \mathrm{NaCl}$ and fixed with $4 \%$ paraformaldehyde (Merck) in phosphate buffered saline for $10 \mathrm{~min}$ at room temperature, washed with water and air dried. After overnight incubation with 1\% Alcian blue (Acros VWR, Belgium) in $0.1 \mathrm{M} \mathrm{HCl}$ at room temperature, cells were washed six times and allowed to air-dry in the dark overnight. Alcian blue was extracted from the cells by incubation with Guanidine-HCl (6 M) (Sigma) for 2 hours under continuous agitation. Extracted Alcian blue was determined spectrophotometrically at 645 $\mathrm{nm}$ using a plate reader (Multiskan FC, Thermo Scientific).

\subsection{Alkaline Phosphatase activity}

Cells were lysed in freshly prepared collection buffer (1.5 M Tris-HCl pH 9.0; $2 \%(\mathrm{v} / \mathrm{v})$ Triton X-100) and homogenized by sonication (Soniprep 150 MSE). Insoluble material was removed by centrifugation $\left(5\right.$ minutes; $\left.13,000 \mathrm{x} \mathrm{g} ; 4^{\circ} \mathrm{C}\right)$. Total protein concentration was determined (see above). ALP activity was determined by in time measuring ALPdepend enzymatic conversion of p-nitrophenyl phosphate to nitrophenyl phosphate in 
buffer containing $1.5 \mathrm{M}$ Tris- $\mathrm{HCl}$; $\mathrm{pH} 9.0,1 \mathrm{mM} \mathrm{ZnCl}_{2}, 1 \mathrm{mM} \mathrm{MgCl}_{2}$ and $7.5 \mathrm{mM}$ p-nitrophenyl phosphate. Substrate conversion was spectrophotometrically quantified at $405 \mathrm{~nm}$. A calibration curve consisted of an increasing concentration of nitrophenyl phosphate to determine the absolute amount of ALP-generated nitrophenyl phosphate in time. Values were normalized to total protein and expressed as $\mu \mathrm{mol}$ nitrophenyl phosphate/minute/gram (=1U).

\subsection{Cell proliferation}

Cells were washed two times with $0.9 \% \mathrm{NaCl}$ and fixated with $4 \%$ paraformaldehyde (Merck) in phosphate buffered saline for $10 \mathrm{~min}$ at room temperature, washed with water and air dried for storage. Cells were incubated with $0.1 \% \mathrm{X}$-violet (Sigma) for $30 \mathrm{~min}$ at room temperature and washed six times with water and allowed to air-dry in the dark overnight. X-violet was extracted from the cells by incubation with $10 \%$ acetic acid for 15 min under continuous agitation. Extracted X-violet was determined spectrophotometrically at $590 \mathrm{~nm}$ using a plate reader (Multiskan FC, Thermo Scientific).

\subsection{Histochemistry}

PACs were decalcified in formalin/EDTA, dehydrated following standard procedures and embedded in paraffin. Before histochemistry, tissue sections $(5 \mu \mathrm{m})$ were deparafinized and rehydrated using standard protocols. Proteoglycans were stained with Safranin-O $(0.1 \%)$ and counterstained with Fast Green $(0.1 \%)$. Stained sections were dehydrated and mounted in Histomount (Thermo Shandon) for microscopic analysis.

\subsection{Statistics}

In the Figures based on ATDC5, bars represent average value of 3 individual experiments (performed in triplicate; $3 \times 3$ samples) and error bars represent mean $\pm 95 \%$ confidence interval (CI). In the experiments using hBMSCs or rPACs, all experiments were performed in triplicate with hBMSCs from 3 independent donors $(n=3)$ or rabbit periosteal explants from 5 independent donors $(n=5)$. Statistical significance $(\mathrm{p}<0.05)$ was determined by unpaired two-tailed student t-tests (paired two-tailed student t-test for data derived from hBMSC experiments (Figure 4.3A)) using GraphPad PRISM 5.0 (La Jolla, CA, USA). To test for normal distribution of the input data, D'Agostino-Pearson omnibus normality tests were performed. All quantitative data sets presented here passed the normality tests. Using an F-test homogeneous variance of the input data was confirmed. 


\section{Results}

\subsection{Differential effects of BMP-2 and BMP-7 on chondrogenic differentiation of progenitor cells}

To determine whether BMP-2 and BMP-7 differently influence the outcome of chondrogenic differentiation of progenitor cells we performed dose-response experiments to establish the minimal BMP concentrations that lead to detectable changes in the chondrogenic outcome. Throughout a 14 day differentiation time course, ATDC5 cells were exposed to increasing equimolar concentrations of either BMP-2 or BMP-7. As presented in Figure 4.1A, 0.01 and $0.1 \mathrm{nM}$ of BMP-2 (grey bars) did not influence the mRNA expression of Collagen type II (Col2a1), although a slight increase was detected at $1.0 \mathrm{nM}$. On the other hand, BMP-7 (black bars) dose-dependently increased Col2al expression. Collagen type X (Col10a1) mRNA expression (Figure 4.1B) revealed a robust dosedependent increase upon BMP-2 exposure, whereas the presence of BMP-7 led to an opposite, Col10a1 suppressing action. From these dose-response experiments we decided to use $1 \mathrm{nM}$ of both BMPs throughout the rest of our experiments, as this dose was the minimal concentration needed to detect BMP-induced changes in chondrogenic gene expression.

A

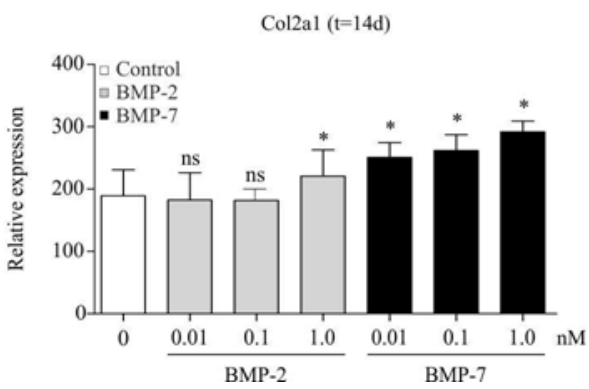

B

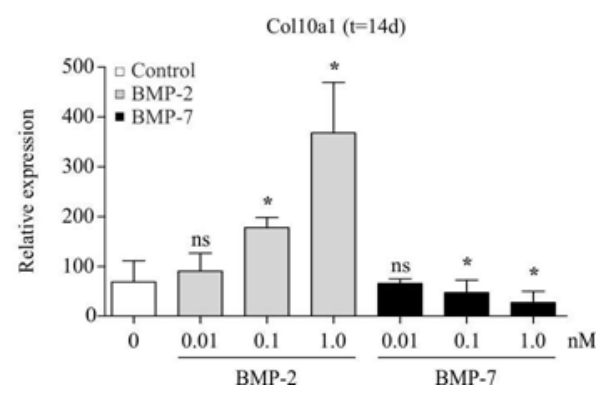

Figure 4.1: Effects of increasing BMP-2 or BMP-7 concentrations on chondrogenic differentiation of ATDC5 cells

Equimolar concentrations $(0,0.01 ; 0.1$ or $1 \mathrm{nM})$ of BMP- 2 or BMP- 7 were added from the start of chondrogenic differentiation of ATDC5. (A). Col2a1 mRNA expression at day 14 in differentiation, relatively to $\mathrm{t}=0$. White bars represent control $(0 \mathrm{nM})$ condition, grey bars the different BMP-2 concentrations and black bars the different BMP-7 concentrations. (B). Same as in (A) but for Col10a1. In graphs, error bars represent mean $\pm 95 \%$ CI. Statistics are calculated with respect to the control condition and $*=p<0.05$ (statistical significant), ns $=$ not stastistical significant. 
To obtain further insight into the action of the different BMPs during the course of chondrogenic differentiation we determined the expression of Col2a1 and Col10a1 at different time points $(0,7,10$ and 14 days) in ATDC5 differentiation. In the course of ATDC5 chondrogenic differentiation a gradual increase was found in Col2a1 and Col10a1 mRNA levels throughout the 14 day timeframe (Figure 4.2A; white bars). Addition of $1 \mathrm{nM}$ BMP-2 (grey bars) resulted in a slight increase in both Col2a1 mRNA and protein synthesis during chondrogenic differentiation (Figure 4.2A/C). Coll0al mRNA and protein levels were higher increased in the BMP-2 condition, as compared to the control condition (Figure 4.2B/C). Addition of an equimolar concentration of BMP-7 (black bars) resulted in increased Col2a1 mRNA and protein levels (Figure 4.2A/C ), whereas Col10a1 mRNA and protein levels were less upregulated under influence of BMP-7 from day 10 on, as compared to control (Figure $4.2 \mathrm{~B} / \mathrm{C}$ ) and as compared to the BMP-2 condition (Figure $4.2 \mathrm{~B} / \mathrm{C}$; black versus grey bars). To further corroborate on the observed pro-chondrogenic / hypertrophy-suppressing action of BMP-7 and the pro-hypertrophic effect of BMP-2 we measured the expression of other important chondrogenic and chondrocyte hypertrophy associated genes. Expression of key chondrogenic transcription factor Sox9 reacted mildly to BMP-2 exposure, whereas challenging the cells with BMP-7 led to higher upregulated Sox9 levels (Figure 4.2C/D). Aggrecan (Acan) gene expression also reacted very similar (to Sox9 and Col2a1) upon exposure to both individual BMPs.

Analogous to our above findings on Col10a1 expression, the expression of other chondrocyte hypertrophy genes as Runx2 (Runt related transcription factor 2), ALP (Alkaline phosphatase) and Mmp13 (Matrix metalloproteinase 13) was found to be increased in the BMP-2 condition (grey bars) as compared to control differentiation conditions (white bars) and suppressed in the BMP-7 condition (black bars) (Figure 4.2C/E). Expression of these hypertrophy-associated genes was also found to be significantly different between the BMP-2 and the BMP-7 condition (Figure 4.2E). A hypertrophy-inducing action of BMP-2 and hypertrophy-suppressive action by BMP-7 was further confirmed by measuring ALP enzymatic activity in these cultures (Figure 4.2F). As an overall indicator for glycosaminoglycan content, alcian blue stainings revealed that both BMPs caused increased glycosaminoglycan content in differentiating ATDC5 cells at day 14, as compared to control differentiation conditions (Figure 4.2G). No differences were detected between the glycosaminoglycan contents of BMP-2 versus BMP-7 treated cells. Interestingly, no significant differences between control, BMP-2 and BMP-7 were found when cell proliferation capacity was determined (Figure $4.2 \mathrm{H}$ ), indicating that both BMPs do not influence 
A

Col2al

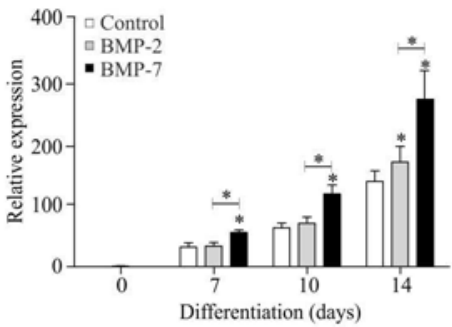

B

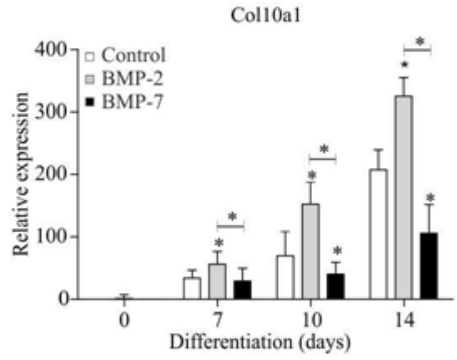

C

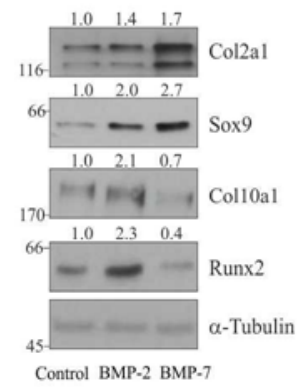

E

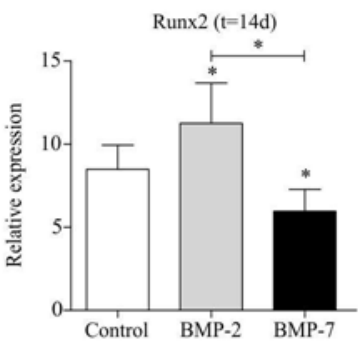

F

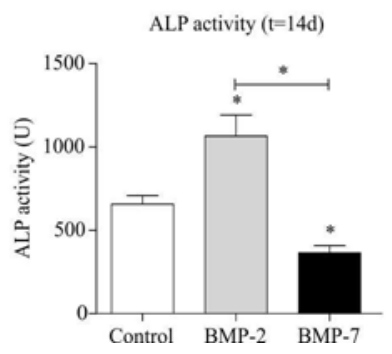

D
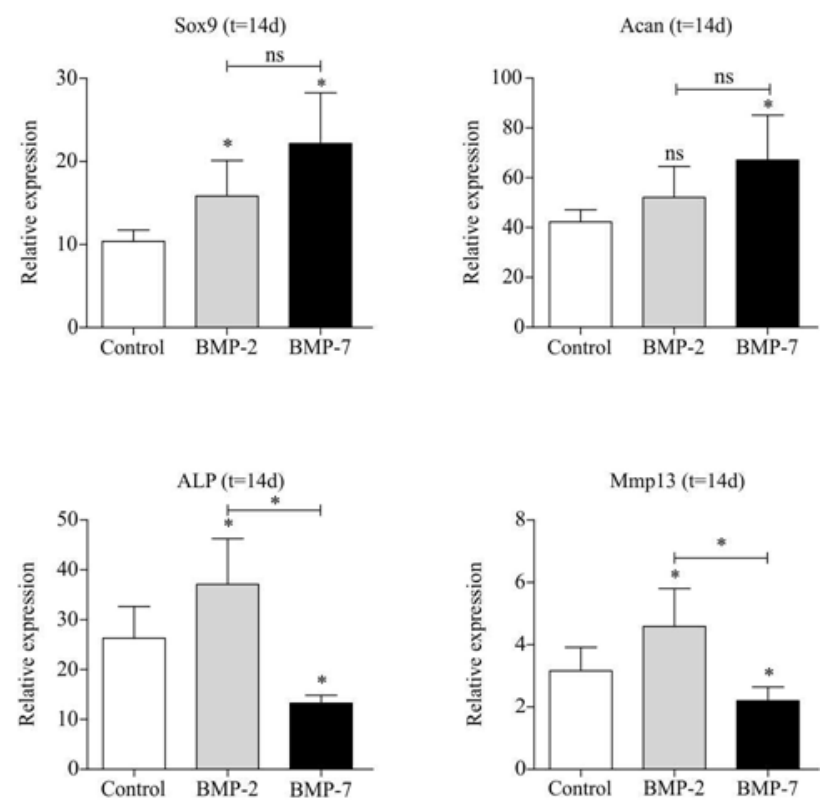

G

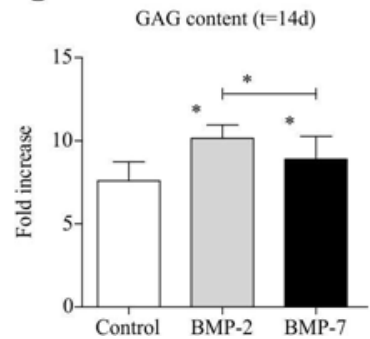

$\mathrm{H}$

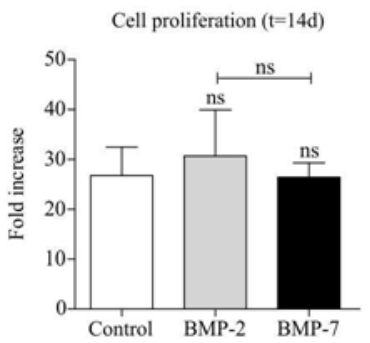


Figure 4.2: Differential effects of BMP-2 and BMP-7 on the chondrogenic outcome of ATDC5 cells Equimolar concentrations (1 nM) of BMP-2 or BMP-7 were added from the start of chondrogenic differentiation of ATDC5. (A). Col2a1 mRNA expression at day 7, 10 and 14 in differentiation, relatively to $\mathrm{t}=0$. White bars represent control condition, grey bars the BMP-2 condition and black bars the BMP-7 condition. (B). Same as in (A) but for Col10a1. (C). Protein expression of Col2a1, Sox9, Col10a1 and Runx2 at day 14 in differentiation. $\alpha$ Tubulin is used as loading control. $(D)$. Induction of Sox9 and Acan mRNA expression was determined at day 14 in differentiation. (E). Same as (D) but for Runx2, ALP and Mmp13 mRNA expression. (F). ALP enzymatic activity $((\mathrm{U}) \mu \mathrm{mol} / \mathrm{g} / \mathrm{min})$ of $\mathrm{t}=14$ samples was determined and normalized to total protein content. $(G)$. Glycosaminoglycan content was determined by Alcian Blue staining and fold increase of $\mathrm{t}=14$ samples was calculated relatively from $\mathrm{t}=0$ samples. $(H)$. Differences in cell proliferation speed between control and BMP conditions in ATDC5 differentiation was determined by X-Violet staining. Fold increase (DNA) from samples from $\mathrm{t}=14$ was calculated relatively to day 0 . In graphs, error bars represent mean $\pm 95 \%$ CI. Statistics are calculated with respect to the control condition and between BMP-2 and BMP-7 conditions. Statistical significant p-values $(\mathrm{p}<0.05)$ are indicated by an *, ns = not stastistical significant. Molecular weight markers $(\mathrm{kDa})$ are depicted on the left of immunoblots and relative quantifications are depicted on top of blots.

the cell proliferation rate in the herein tested setting.

Finally, to verify our ATDC5 findings in primary human mesenchymal progenitor cells, we supplemented the differentiation medium of hBMSCs with equimolar concentrations $(1 \mathrm{nM})$ of either two BMPs. Chondrogenic differentiation of three individual hBMSC isolates led to upregulation of COL2A1, COL10A1 and RUNX2 mRNAs at day 14 and 21 in differentiation (Figure 4.3A; white bars). The addition of $1 \mathrm{nM}$ BMP-2 increased the mRNA expression of COL10A1 and RUNX2, while leaving COL2A1 mRNA levels almost unchanged (Figure 4.3A; grey bars). However, addition of $1 \mathrm{nM} \mathrm{BMP-7}$ to the differentiation medium resulted in significantly increased COL2A1 mRNA expression, while COL10A1 and RUNX2 mRNA expression was suppressed at day 21 (Figure 4.3A; black bars). Immunoblot analysis of protein lysates from day 21 samples overall confirmed the above findings (Figure 4.3B). To establish the opposing actions of BMP-2 and BMP-7 in cartilage tissue formation we used an ex vivo periosteal organ culture system able to differentiate into cartilaginous tissue ${ }^{26,27}$. The mesenchymal progenitor cell-containing periosteal tissue was differentiated in between agarose layers (periosteum agarose culture (PAC) under influence of TGF $\beta 3$ and generated cartilaginous tissue after 20 days in differentiation, as visualized by safranin-O staining of tissue sections (Figure 4.3C). The cartilaginous tissue expressed Col2a1, Col10a1 and Runx2 mRNAs (Figure 4.3D). BMP-2 supplementation showed an increased hypertrophic phenotype (Figure 4.3C) as well as increased Col2a1, Col10a1 and Runx2 gene expression (grey bars). On the contrary, BMP- 
7 specifically increased Col2a1 mRNA expression, but showed a decreased Col10a1 and Runx2 mRNA expression as opposed to the control and BMP-2 conditions (black bars).

Together these data show that exposure to equimolar concentrations of BMP-2 or BMP-7 evokes differential chondrogenic outcomes during differentiation of ATDC5 cells, hBMSCs and rabbit periosteal explants; BMP-2 stimulates chondrocyte hypertrophy, whereas BMP-7 suppresses chondrocyte hypertrophy in the herein tested equimolar concentrations.

A
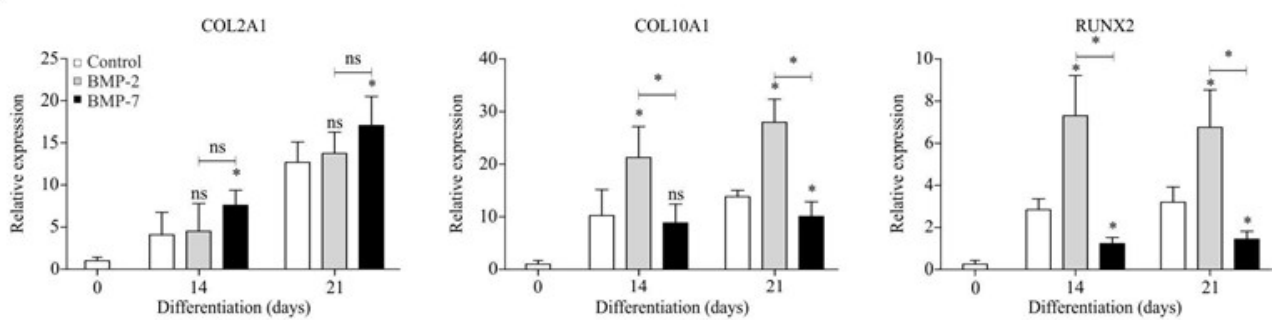

B

$\mathrm{C}$

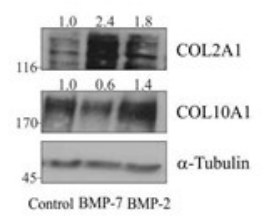

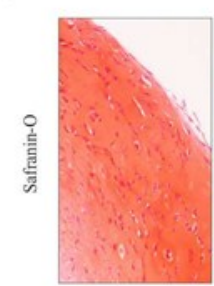

Control

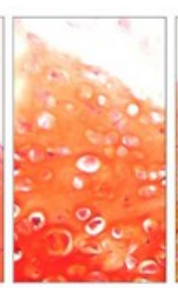

BMP-2

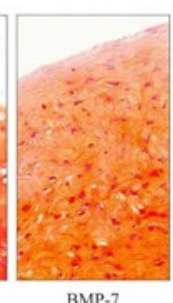

BMP-7

$\mathrm{D}$
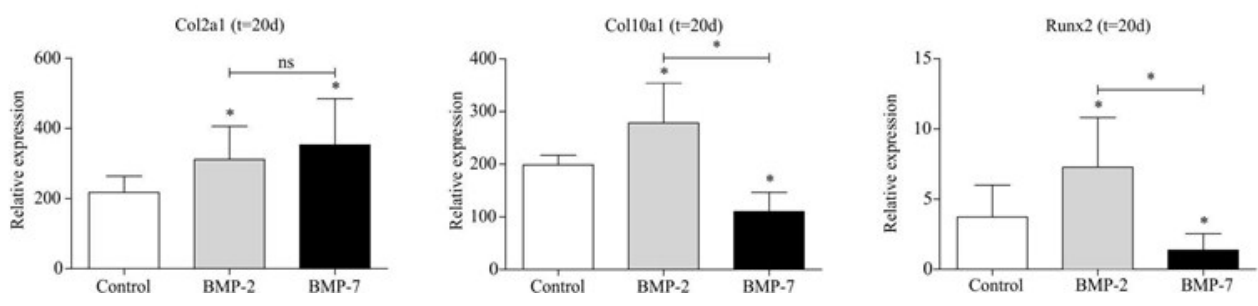

Figure 4.3: Differential effects of BMP-2 and BMP-7 on chondrogenic differentiation of hBMSCs and rabbit periosteal explants

Equimolar concentrations (1 nM) of BMP-2 or BMP-7 were added from the start of chondrogenic differentiation of hBMSCs ( $\mathrm{n}=3$ individual donors) or rPACs ( $\mathrm{n}=5$ individual donors). (A). COL2A1, COL10A1 and RUNX2 mRNA expression at day 14 and 21 in hBMSC chondrogenic differentiation, relatively to $\mathrm{t}=0$. White bars represent control condition, grey bars the BMP-2 treated condition and black bars the BMP-7 condition. (B). Protein expression of COL2A1 and COL10A1 at day 21 in chondrogenic differentiation of hBMSCs. $\alpha$-Tubulin is used as loading control and molecular weight markers $(\mathrm{kDa})$ are depicted on the left. $(C)$. rPAC's were harvested after 20 days of chondrogenic differentiation. The panel shows representative examples of rPAC tissue sections 
stained with Safranin-O/Fast Green. Magnification is 200x. (D). Col2a1, Col10a1 and Runx2 mRNA expression at day 20 in chondrogenic differentiation or rPACs, relatively to $\mathrm{t}=0$. White bars represent control condition, grey bars the BMP-2 treated condition and black bars the BMP-7 condition. In graphs, bars represent the average value of triplicate samples of 3 individual BMSC donors $(n=3)$ or triplicate samples of 5 individual rPAC donors $(n=5)$ and error bars represent mean $\pm 95 \%$ CI. Statistics are calculated with respect to the control condition and between BMP-2 and BMP-7 conditions. Statistical significant $\mathrm{p}$-values $(\mathrm{p}<0.05)$ are indicated by an $*$, ns $=$ not stastistical significant.

\subsection{Involvement of Bapx1/Nkx3.2 in mediating the hypertrophy suppressing action of BMP}

\section{-7 in ATDC5 cells}

As BMP-2 and BMP-7 display differential effects on the chondrogenic outcome of progenitor cells it is expected that both BMPs differently direct pathways that balance the chondrogenic/hypertrophic fate of these cells. An important regulator in determining the chondrogenic fate is $\operatorname{Bapx} 1 / \mathrm{Nkx} 3.2^{28,29}$. This transcription factor has been linked to preventing chondrocyte hypertrophy and maintaining a chondrogenic phenotype $\mathrm{e}^{28,30,31}$. To determine whether Bapx1/Nkx3.2 may play a role as a downstream effector in the differential actions of BMP-2 and BMP-7 during chondrogenic differentiation, we measured Bapx1/Nkx3.2 expression levels in similar samples from Figure 4.2. As previously reported $^{30}$, upregulation of Bapx1/Nkx3.2 expression was inversely correlated with progression in chondrogenic differentiation (Figure 4.4A; white bars). Expression of Bapx1/Nkx3.2 hardly responded to the presence of $1 \mathrm{nM}$ BMP-2 (Figure 4.4A; grey bars and immunoblot). In contrast, an equimolar concentration of BMP-7 caused a significantly increased expression of Bapx1/Nkx3.2 at every time point measured in chondrogenic differentiation (Figure 4.4A; black bars and immunoblot), indeed suggesting an involvement in the hypertrophy-suppressing action of BMP-7. To further investigate a potential functional involvement of Bapx1/Nkx3.2 in the BMP-7 action, Bapx1/Nkx3.2 expression was targeted in ATDC5 cells by RNAi. Transfection of a Bapx1/Nkx3.2 siRNA duplex during the course of chondrogenic differentiation (in the absence of added BMP-7) showed knockdown of Bapx1/Nkx3.2 mRNA as well as its protein expression at ten days in ATDC5 chondrogenic differentiation (Figure 4.4B; black bar and immunoblot). Previously, transcription of Bapx1/Nkx3.2 was reported to be driven by $\operatorname{Sox} 9^{28}$. In accordance with this we found that Sox 9 expression only slightly, but not significantly, responded to Bapx1/Nkx3.2 knockdown (Figure 4.4B; black bar and immunoblot), as did 
A Bapx1/ Nkx3.2

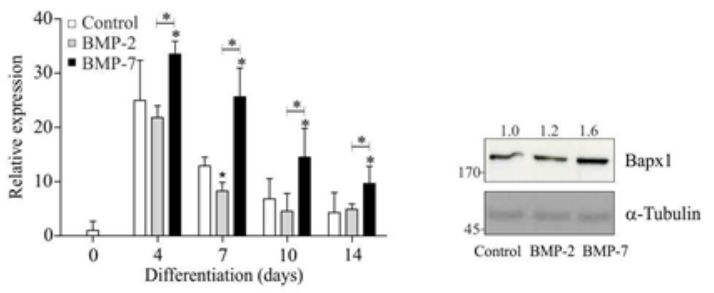

B
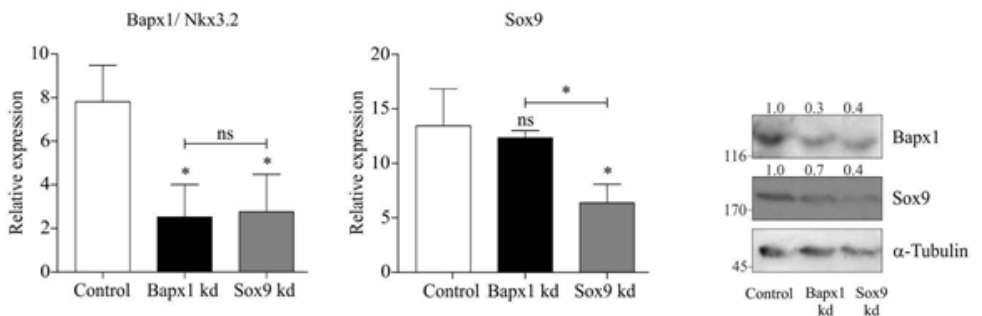

$\mathrm{C}$
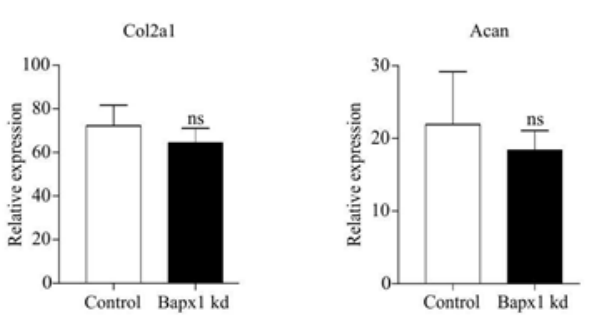

E

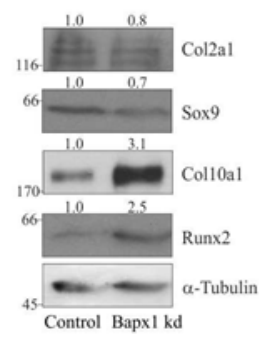

D
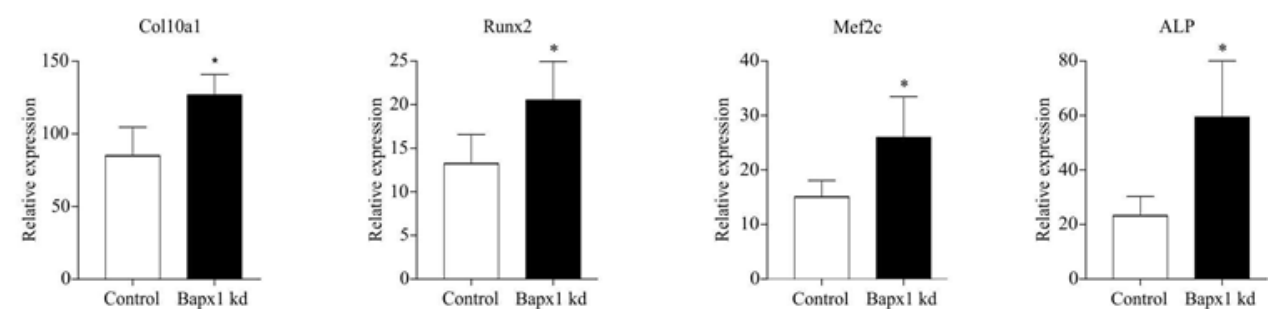

Figure 4.4: Bapx1/Nkx3.2 is involved in regulating the hypertrophic fate of ATDC5 cells

(A) Bapx1/Nkx3.2 mRNA expression was determined in similar samples as Figure 4.2A/B. Right panel shows protein expression of Bapx1/Nkx3.2 at day 14 in ATDC5 differentiation in the absence or presence of equimolar concentrations of BMP-2 or BMP-7 (1 nM). (B). RNAi mediated knockdown of Bapx1/Nkx3.2 (black bars) or Sox9 (grey bars) mRNA expression was confirmed at day 10 in differentiation. Protein expression in similar samples is shown in the right panel. $(C)$. Expression of Col2a1 and Acan mRNAs was determined in Mock and Bapx1/Nkx3.2 knockdown cells (B) at day 10 in differentiation. (D). In samples from (C) but for Runx2, Col10a1, Mef2c and ALP mRNA. (E). Protein expression of Col2a1, Sox9, Col10a1 and Runx2 in Mock and 
Bapx1/Nkx3.2 knockdown cells at day 10 in differentiation. In graphs, error bars represent mean $\pm 95 \%$ CI. Statistics are calculated with respect to the control condition $(*$ indicates p-values $<0.05, \mathrm{~ns}=$ not statistical significant), between BMP-2 and BMP-7 conditions and between Bapx1/Nkx3.2 and Sox9 siRNA treated conditions. For immunoblots, $\alpha$-Tubulin is used as loading control, molecular weight markers $(\mathrm{kDa})$ are depicted on the left of immunoblots and relative quantifications are depicted on top of blots.

Sox9 transcriptional targets Col2a1 and Acan (Figure 4.4C/E). On the other hand, knockdown of Sox9 (Figure 4.4B; grey bar) downregulated the expression of Bapx1/Nkx3.2 to the same extend as the dedicated Bapx1/Nkx3.2 knockdown, confirming that Sox 9 mainly acts upstream of Bapx1/Nkx3.2 expression. Messenger RNA and protein expression of the Bapx1/Nkx3.2 repressional target Runx2 was increased upon Bapx1/Nkx3.2 knockdown (Figure 4.4D/E). Along with Runx2 other important chondrocyte hypertrophy-associated genes Col10a1, Mef2c (Myocyte-specific enhancer factor 2c) and ALP expression were also upregulated when expression of Bapx1/Nkx3.2 was inhibited (Figure 4.4D/E). We subsequently determined if the hypertrophy-suppressing effect of BMP-7 during chondrogenic differentiation is related to the above Bapx1/Nkx3.2 action. To address this question we now transfected differentiating ATDC5 cells with the Bapx1/Nkx3.2 siRNA duplex in the absence or presence of $1 \mathrm{nM}$ BMP-7. The presence of BMP-7 faithfully increased Bapx1/Nkx3.2 mRNA expression (Figure 4.5A; black bar) and the Bapx1/Nkx3.2 siRNA duplex effectively downregulated the BMP-7-induced Bapx1/Nkx3.2 expression (Figure 4.5A; grey bar versus black bar). In accordance with the results presented in Figure 4.4B, Bapx1/Nkx3.2 knockdown in the presence of BMP-7 did not significantly affect Sox9 mRNA expression, and only a slight, but not significant, decrease in Col2a1 and Acan mRNA expression was observed (Figure 4.5B). In contrast, we did observe clear effects on expression of chondrocyte hypertrophic genes. Despite the presence of BMP-7, Bapx1/ Nkx3.2 knockdown completely abolished the hypertrophy-suppressing effect of BMP-7. Expression levels of Col10a1, Runx2, Mef2c and ALP returned back to control conditionlevels or even higher (Figure 4.5C/D), showing that Bapx1/Nkx3.2 is involved in the hypertrophy-suppressing action of BMP-7 during chondrogenic differentiation of ATDC5 cells.

In conclusion, we here show that BMP-2 and BMP-7 have differential effects on the chondrogenic outcome of differentiating chondroprogenitor cells: BMP-2 acts a specific inducer of chondrocyte hypertrophy, while BMP-7 appears to increase or maintain chondrogenic potential and is able to suppress the level of chondrocyte hypertrophy via Bapx1/Nkx3.2. 
A
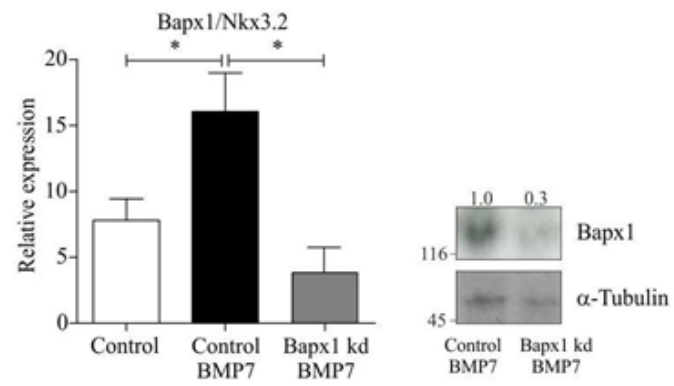

B
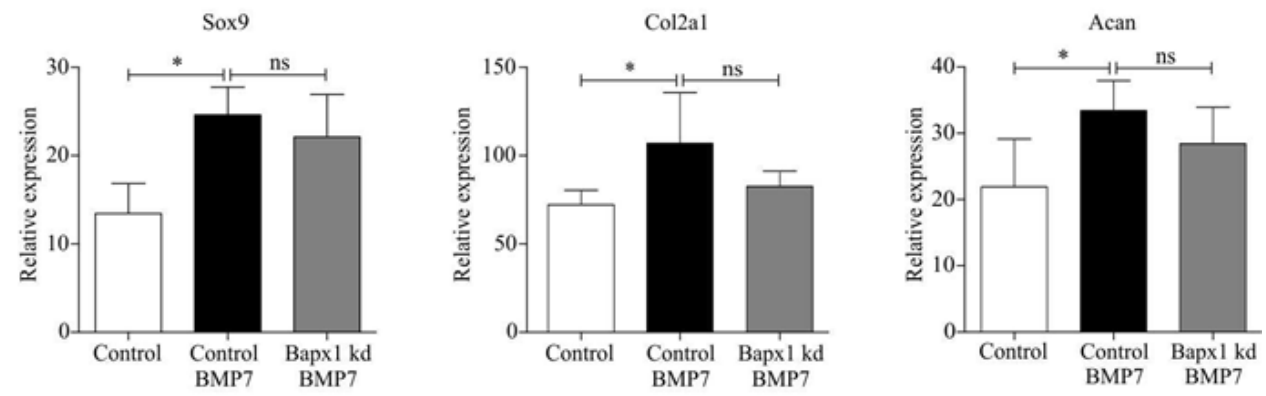

C
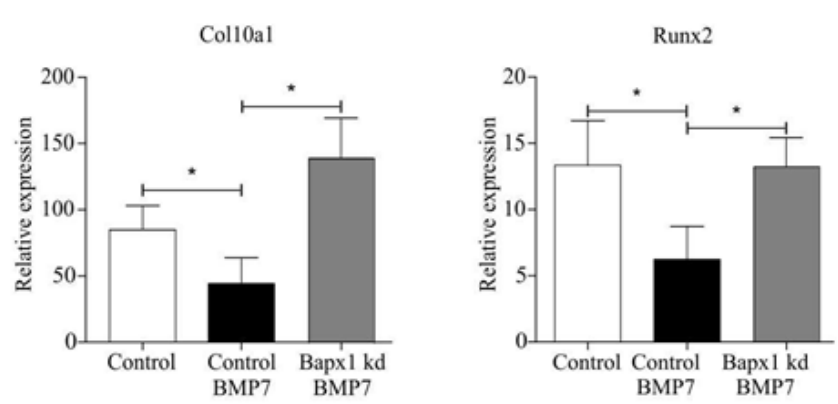

D
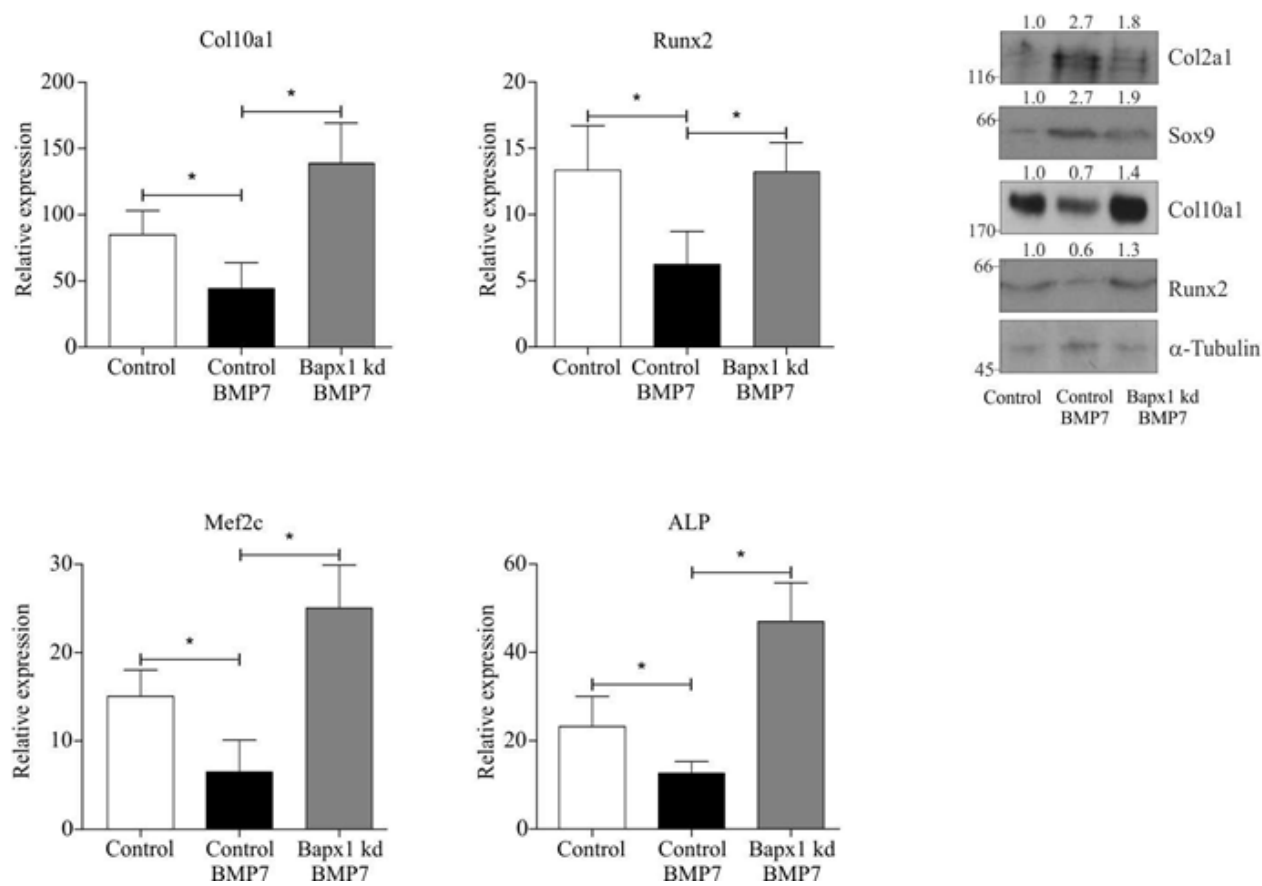
Figure 4.5: Bapx1/Nkx3.2 is involved in the hypertrophy-suppressing action of BMP-7 in ATDC5 cells

The involvement of Bapx1/Nkx3.2 in the hypertrophy-suppressing action of BMP-7 during chondrogenic differentiation of ATDC5 cells was determined. $(A)$. Knockdown of Bapx1/Nkx3.2 by siRNA transfections was confirmed at day 10 in differentiation of BMP-7 treated cells on mRNA (left panel, grey bars) and protein level (right panel). (B). Expression of Sox9, Col2al and Acan mRNAs was determined in Mock (white and black bars, without/with BMP-7 respectively) and Bapx1/Nkx3.2 knockdown cells (grey bars) at day 10 in differentiation in BMP -7 treated cells. (C). In samples from (B.) mRNA expression of Col10a1, Runx2, Mef2c and ALP was determined. (D). Protein expression of Col2a1, Sox9, Col10a1 and Runx2 in Mock and Bapx1/Nkx3.2 knockdown cells at day 10 in differentiation of BMP-7 treated cells. In graphs, error bars represent mean $\pm 95 \%$ CI. Statistics are calculated with respect to the control condition and BMP-7 control condition, as well as between the BMP-7 control condition and the BMP-7/Bapx1 kd condition, Statistical significant p-values $(\mathrm{p}<0.05)$ are shown by an *, ns = not statistical significant. For immunoblots, $\alpha$-Tubulin is used as loading control, molecular weight markers $(\mathrm{kDa})$ are depicted on the left of immunoblots and relative quantifications are depicted on top of blots.

\section{Discussion}

Although BMP-2 and BMP-7 are both clinically used in bone fracture repair and are potential targets in cartilage regenerative approaches, a comparison of the effects of BMP-2 versus BMP-7 on chondrogenic differentiation of progenitor cells in vitro is not well documented and not taking chondrocyte hypertrophy into account. We here report that BMP-2 and BMP-7 display opposing actions on the outcome of chondrogenic differentiation of progenitor cells; BMP-2 stimulates a hypertrophic chondrocyte phenotype, whereas BMP-7 appears to suppress chondrocyte hypertrophy during chondrogenic differentiation, associated with an increased chondrogenic phenotype. A hypertrophy stimulating action was reported previously for BMP- $2^{26}$, whereas the hypertrophysuppressing action of BMP-7 is an exciting new finding. Chubinskaya and colleagues reported that BMP-2 but even more BMP-7 was able to increase proteoglycan synthesis in adult human articular chondrocytes cultured in alginate beads ${ }^{14}$. This indeed suggests that BMP-7 predominantly acts in favour of a chondrogenic phenotype. Data reported by Shintani et al., are supporting our findings. The authors reported on a more pronounced beneficial action of BMP-7 as opposed to BMP-2 on chondrogenic outcome of bovine synovial explants, but hardly addressed the effects on chondrocyte hypertrophy ${ }^{32}$. Similarly, Kurth and colleagues compared different TGF $\beta$-superfamily members and other factors for their chondrogenic potential on human synovial mesenchymal stem cells, but also excluded hypertrophic genes from their analyses ${ }^{33}$. In addition, Knippenberg et al. 
showed that short-term treatment (15 minutes) of undifferentiated mesenchymal progenitor cells with BMP-2 resulted in differentiation towards the osteogenic lineage, whereas shortterm BMP-7 treatment increased chondrogenic marker expression ${ }^{34}$. The majority of the literature however reports on the individual effects of BMP-2 or BMP-7 on chondrogenic outcome of progenitor cell differentiation ${ }^{16,23,33,35-37}$.

Studies reporting on the use of BMPs in in vitro and ex vivo cell/cartilage models used various concentrations of both BMPs ranging from $10 \mathrm{ng} / \mathrm{ml}^{34}$ to as high as $500 \mathrm{ng} / \mathrm{ml}^{38}$. The BMP concentrations that were tested in our dose response experiments (Figure 4.1) are relatively low as compared to the majority of the published studies. As our main aim was to investigate whether BMP-2 and BMP-7 may display differential actions, we hypothesized that an inverse limiting dilution approach would be the most sensitive way to compare both BMPs, while avoiding the risk of growth factor overload and potential aspecific use of BMP receptors ${ }^{39,40}$. It remains however to be determined how higher concentrations of both BMPs would compare in their chondrogenic actions on the herein tested chondrogenic models.

In analogy to the process of chondrogenic differentiation, different BMPs are expressed in different zones of the growth plate and it is thus likely that these BMPs exert differentiation-phase dedicated functions in regulating chondrogenic development. BMP-2 is expressed by hypertrophic chondrocytes whereas BMP-7 is mainly expressed by proliferating chondrocytes ${ }^{41}$. Also the BMP type I receptors, which activate R-Smad signalling, display differential expression patterns in the developing growth plate. BMP receptor type IA (BMPRIA or ALK (activin receptor-like kinase) 3) is expressed by proliferating and hypertrophic chondrocytes, BMPR-IB (also known as ALK6) is found throughout the growth plate and ALK2 is detected in the resting zone and proliferating chondrocytes. These receptors do not bind all BMPs with similar affinity and can thus potentially induce different chondrogenic outcomes. BMP-2 preferentially binds to BMPR-IA and BMPR-IB, whereas BMP-7 binds with higher affinity to ALK2 and BMPR-IB ${ }^{39,41,42}$, which could suggest a more prominent function for BMP-2 in chondrocyte hypertrophy and for BMP-7 in chondrocyte maintenance and cartilaginous extracellular matrix synthesis. Although previously specific Smad-signalling has been linked to the chondrogenic fate ${ }^{43}$, it remains elusive whether the herein found differential action of BMP-2 and BMP-7 can be explained by specific Smad activation. In addition BMPs can also induce signalling through MAP (mitogen activated protein) kinases and thereby influence the chondrogenic outcome ${ }^{6}$. We are currently evaluating the above options. 
As a link between BMP-2 and chondrocyte hypertrophy has been established previously ${ }^{26,44}$ we here specifically further focussed on a possible mechanism which could explain the hypertrophy-suppressing action of BMP-7. Focussing on Sox9 and Runx2 as key factors in regulating the balance between chondrogenic maintenance and chondrocyte hypertrophy, it was reported previously that Sox9 is able to repress Runx2 expression via the Runx2 transcriptional repressor Bapx1/Nkx3.2 $28,30,31$. Bapx1/Nkx3.2 is a transcriptional target of Sox 9 and thus the BMP-7 induced Sox9 levels may prevent Runx2 induction via upregulating Bapx1/Nkx3.28 . In addition to suppressing the transcription of Runx2, Bapx1/Nkx3.2 was recently found to be directly involved in driving the transcription of Col2a1 $1^{45}$, providing an potential alternative route via which the BMP-7-induced Bapx1/Nkx3.2 may balance the hypertrophic fate of a chondrocyte. However, knockdown of Bapx1/Nkx3.2 in ATDC5 showed only moderate/non-significant effects on Col2a1 mRNA levels, making it conceivable that the hypertrophy suppressive action of BMP-7 via Bapx1/Nkx3.2 in ATDC5 is mainly via an active hypertrophy-suppressing route rather than an active pro-chondrogenic (Col2a1) mechanism. Another possible determinant in the Bapx1/Nkx3.2 depending BMP-7 action could be parathyroid hormone related peptide (PTHrP). Our unpublished data show that PTHrP expression is upregulated upon exposure to BMP-7. Previously it has been reported that maintenance of Bapx1/Nkx3.2 expression in growth plate proliferative chondrocytes depends on PTHrP signals ${ }^{31}$ and that BMP and PTHrP signalling interact ${ }^{16}$. Finally, sonic hedgehog (Shh) has been reported to induce Bapx1/Nkx3.2 expression to induce somitic chondrogenesis in avian progenitor cells ${ }^{46}$. It was found that Nkx3.2 (like Shh) can only induce somitic chondrogenesis in the presence of a BMP signal. These findings may provide alternative explanations through which BMP -7 might execute its hypertrophy-suppressive action, although at this stage it is not clear what the potential relative contribution of these alternative pathways is to control expression of Bapx1/Nkx3.2. Furthermore, it is well known that Sox9 is regulated by BMP activity in vitro and in vivo ${ }^{17,47,48}$. This was confirmed in our study for BMP-2 and BMP-7. In view of a Sox9-dependent expression of Bapx1/Nkx3.2 it was therefore surprising that we did not measure induction of Bapx1/Nkx3.2 in both BMP conditions. This discrepancy may be explained by potential BMP-specific differential signalling pathways downstream of different BMP-receptors, allowing (Sox9-driven) Bapx1/Nkx3.2 expression in the BMP-7 conditions, while not supporting this upon signalling by BMP-2. This, however, remains speculation. 
In conclusion, we here show that BMP-2 and BMP-7 have differential actions during chondrogenic differentiating of chondroprogenitor cells: BMP-2 acts a specific inducer of chondrocyte hypertrophy, while BMP-7 appears to increase or maintain chondrogenic potential and prevent chondrocyte hypertrophy. These results may provide a rationale for an application-dependent use of specific BMPs in endochondral-based cartilage and bone regenerative techniques ${ }^{49}$.

\section{Acknouledgements}

The authors thank the Dutch Arthritis Association (grant LLP14), the Dutch Stichting Annafonds|NOREF (grants 07/07 and 08/42) and the Kootstra Talent Fellowship for their financial support. 


\section{References}

1 Kronenberg, H. M. Developmental regulation of the growth plate. Nature 423, 332-336 (2003).

2 Mackie, E. J., Ahmed, Y. A., Tatarczuch, L., Chen, K. S. \& Mirams, M. Endochondral ossification: how cartilage is converted into bone in the developing skeleton. Int J Biochem Cell Biol 40, 46-62 (2008).

3 Dickhut, A. et al. Calcification or dedifferentiation: requirement to lock mesenchymal stem cells in a desired differentiation stage. J Cell Physiol 219, 219-226 (2009).

4 van Osch, G. J. et al. Cartilage repair: past and future--lessons for regenerative medicine. Journal of cellular and molecular medicine 13, 792-810 (2009).

5 Farrell, E. et al. Chondrogenic priming of human bone marrow stromal cells: a better route to bone repair? Tissue engineering. Part C, Methods 15, 285-295, doi:10.1089/ten.tec.2008.0297 (2009).

6 Derynck, R. \& Zhang, Y. E. Smad-dependent and Smad-independent pathways in TGF-beta family signalling. Nature 425, 577-584, doi:10.1038/nature02006 (2003).

7 Johnstone, B., Hering, T. M., Caplan, A. I., Goldberg, V. M. \& Yoo, J. U. In vitro chondrogenesis of bone marrow-derived mesenchymal progenitor cells. Experimental cell research 238, 265-272, doi:10.1006/excr.1997.3858 (1998).

8 Urist, M. Biogenesis of bone: Calcium and phophorus in the skeleton and blood in vertebrate evolution. (Am. Phys. Soc., 1976).

9 Bessa, P. C., Casal, M. \& Reis, R. L. Bone morphogenetic proteins in tissue engineering: the road from laboratory to clinic, part II (BMP delivery). Journal of tissue engineering and regenerative medicine 2, 81-96, doi:10.1002/term.74 (2008).

10 Bessa, P. C., Casal, M. \& Reis, R. L. Bone morphogenetic proteins in tissue engineering: the road from the laboratory to the clinic, part I (basic concepts). Journal of tissue engineering and regenerative medicine 2, 1-13, doi:10.1002/term.63 (2008).

11 Xiao, Y. T., Xiang, L. X. \& Shao, J. Z. Bone morphogenetic protein. Biochemical and biophysical research communications 362, 550-553, doi:10.1016/ j.bbrc.2007.08.045 (2007).

12 Pizette, S. \& Niswander, L. BMPs are required at two steps of limb chondrogenesis: formation of prechondrogenic condensations and their differentiation into chondrocytes. Dev Biol 219, 237-249, doi:10.1006/dbio.2000.9610 (2000).

13 van der Kraan, P. M., Blaney Davidson, E. N. \& van den Berg, W. B. Bone morphogenetic proteins and articular cartilage: To serve and protect or a wolf in sheep clothing's? Osteoarthritis and cartilage / OARS, Osteoarthritis Research Society 18, 735-741, doi:10.1016/j.joca.2010.03.001 (2010).

14 Chubinskaya, S., Segalite, D., Pikovsky, D., Hakimiyan, A. A. \& Rueger, D. C. Effects induced by BMPS in cultures of human articular chondrocytes: comparative studies. Growth Factors 26, 275-283, doi:10.1080/08977190802291733 (2008).

15 Erickson, D. M. et al. Recombinant bone morphogenetic protein (BMP)-2 regulates costochondral growth plate chondrocytes and induces expression of BMP-2 and BMP-4 in a cell maturation-dependent manner. J Orthop Res 15, 371-380, 
doi:10.1002/jor.1100150309 (1997).

16 Minina, E. et al. BMP and $\mathrm{Ihh} / \mathrm{PTHrP}$ signaling interact to coordinate chondrocyte proliferation and differentiation. Development 128, 4523-4534 (2001).

17 Chimal-Monroy, J. et al. Analysis of the molecular cascade responsible for mesodermal limb chondrogenesis: Sox genes and BMP signaling. Dev Biol 257, 292301 (2003).

18 Pan, Q. et al. Sox9, a key transcription factor of bone morphogenetic protein-2induced chondrogenesis, is activated through BMP pathway and a CCAAT box in the proximal promoter. Journal of cellular physiology 217, 228-241, doi:10.1002/ jcp.21496 (2008).

19 Chubinskaya, S., Hurtig, M. \& Rueger, D. C. OP-1/BMP-7 in cartilage repair. International orthopaedics 31, 773-781, doi:10.1007/s00264-007-0423-9 (2007).

20 Fan, Z. et al. Regulation of anabolic and catabolic gene expression in normal and osteoarthritic adult human articular chondrocytes by osteogenic protein-1. Clinical and experimental rheumatology 22, 103-106 (2004).

21 Flechtenmacher, J. et al. Recombinant human osteogenic protein 1 is a potent stimulator of the synthesis of cartilage proteoglycans and collagens by human articular chondrocytes. Arthritis and rheumatism 39, 1896-1904 (1996). Nishihara, A., Fujii, M., Sampath, T. K., Miyazono, K. \& Reddi, A. H. Bone morphogenetic protein signaling in articular chondrocyte differentiation. Biochemical and biophysical research communications 301, 617-622 (2003).

Klein-Nulend, J. et al. Osteogenic protein (OP-1, BMP-7) stimulates cartilage differentiation of human and goat perichondrium tissue in vitro. Journal of biomedical materials research 40, 614-620 (1998).

Caron, M. M. et al. Activation of NF-kappaB/p65 Facilitates Early Chondrogenic Differentiation during Endochondral Ossification. PLoS One 7, e33467, doi:10.1371/journal.pone.0033467 (2012).

5 Schallmoser, K. et al. Rapid large-scale expansion of functional mesenchymal stem cells from unmanipulated bone marrow without animal serum. Tissue engineering 14, 185-196 (2008).

26 Welting, T. J. et al. Inhibition of cyclooxygenase-2 impacts chondrocyte hypertrophic differentiation during endochondral ossification. European cells \& materials 22, 420-436; discussion 436-427 (2011).

27 O'Driscoll, S. W., Recklies, A. D. \& Poole, A. R. Chondrogenesis in periosteal explants. An organ culture model for in vitro study. J Bone Joint Surg Am 76, 1042-1051 (1994).

Yamashita, S. et al. Sox9 directly promotes Bapx1 gene expression to repress Runx2 in chondrocytes. Experimental cell research 315, 2231-2240, doi:10.1016/ j.yexcr.2009.03.008 (2009).

Zeng, L., Kempf, H., Murtaugh, L. C., Sato, M. E. \& Lassar, A. B. Shh establishes an Nkx3.2/Sox9 autoregulatory loop that is maintained by BMP signals to induce somitic chondrogenesis. Genes Dev 16, 1990-2005, doi:10.1101/ gad.1008002 (2002).

30 Lengner, C. J. et al. Nkx3.2-mediated repression of Runx2 promotes chondrogenic differentiation. The Journal of biological chemistry 280, 15872-15879, doi:10.1074/jbc.M411144200 (2005).

31 Provot, S. et al. Nkx3.2/Bapx1 acts as a negative regulator of chondrocyte maturation. Development 133, 651-662, doi:10.1242/dev.02258 (2006).

32 Shintani, N. \& Hunziker, E. B. Chondrogenic differentiation of bovine synovium: bone morphogenetic proteins 2 and 7 and transforming growth factor betal induce the formation of different types of cartilaginous tissue. Arthritis and rheu- 
matism 56, 1869-1879, doi:10.1002/art.22701 (2007).

33 Kurth, T. et al. Chondrogenic potential of human synovial mesenchymal stem cells in alginate. Osteoarthritis and cartilage / OARS, Osteoarthritis Research Society 15, 1178-1189, doi:10.1016/j.joca.2007.03.015 (2007).

34 Knippenberg, M., Helder, M. N., Zandieh Doulabi, B., Wuisman, P. I. \& KleinNulend, J. Osteogenesis versus chondrogenesis by BMP-2 and BMP-7 in adipose stem cells. Biochemical and biophysical research communications 342, 902-908, doi:10.1016/j.bbrc.2006.02.052 (2006).

35 Fischer, L., Boland, G. \& Tuan, R. S. Wnt signaling during BMP-2 stimulation of mesenchymal chondrogenesis. Journal of cellular biochemistry 84, 816-831, doi:10.1002/jcb.10091 [pii] (2002).

36 Shen, B., Wei, A., Tao, H., Diwan, A. D. \& Ma, D. D. BMP-2 Enhances TGFbeta3-Mediated Chondrogenic Differentiation of Human Bone Marrow Multipotent Mesenchymal Stromal Cells in Alginate Bead Culture. Tissue Eng Part A 15, 1311-1320 (2009).

37 Shukunami, C., Ohta, Y., Sakuda, M. \& Hiraki, Y. Sequential progression of the differentiation program by bone morphogenetic protein-2 in chondrogenic cell line ATDC5. Experimental cell research 241, 1-11, doi:10.1006/excr.1998.4045 (1998).

38 Sekiya, I., Larson, B. L., Vuoristo, J. T., Reger, R. L. \& Prockop, D. J. Comparison of effect of BMP-2, -4 , and -6 on in vitro cartilage formation of human adult stem cells from bone marrow stroma. Cell and tissue research 320, 269-276, doi:10.1007/s00441-004-1075-3 (2005).

39 Miyazono, K., Maeda, S. \& Imamura, T. BMP receptor signaling: transcriptional targets, regulation of signals, and signaling cross-talk. Cytokine Growth Factor Rev 16, 251-263, doi:S1359-6101(05)00039-0 [pii] 10.1016/j.cytogfr.2005.01.009 (2005).

40 van der Kraan, P. M., Blaney Davidson, E. N., Blom, A. \& van den Berg, W. B. TGF-beta signaling in chondrocyte terminal differentiation and osteoarthritis: modulation and integration of signaling pathways through receptor-Smads. Osteoarthritis and cartilage / OARS, Osteoarthritis Research Society 17, 1539-1545, doi:10.1016/j.joca.2009.06.008 (2009).

41 Pogue, R. \& Lyons, K. BMP signaling in the cartilage growth plate. Curr Top Dev Biol 76, 1-48, doi:10.1016/S0070-2153(06)76001-X (2006).

42 Zou, H., Wieser, R., Massague, J. \& Niswander, L. Distinct roles of type I bone morphogenetic protein receptors in the formation and differentiation of cartilage. Genes Dev 11, 2191-2203 (1997).

43 Hellingman, C. A. et al. Smad Signaling Determines Chondrogenic Differentiation of Bone-Marrow-Derived Mesenchymal Stem Cells: Inhibition of Smad1/5/8P Prevents Terminal Differentiation and Calcification. Tissue Eng Part $A$, doi:10.1089/ten.TEA.2010.0043 (2011).

44 Steinert, A. F. et al. Hypertrophy is induced during the in vitro chondrogenic differentiation of human mesenchymal stem cells by bone morphogenetic protein-2 and bone morphogenetic protein-4 gene transfer. Arthritis Res Ther 11, R148 (2009).

45 Kawato, Y. et al. Nkx3.2 promotes primary chondrogenic differentiation by upregulating Col2a1 transcription. PLoS One 7, e34703, doi:10.1371/ journal.pone.0034703 (2012).

46 Murtaugh, L. C., Zeng, L., Chyung, J. H. \& Lassar, A. B. The chick transcriptional repressor Nkx3.2 acts downstream of Shh to promote BMP-dependent axial chondrogenesis. Dev Cell 1, 411-422 (2001). 
47 Yoon, B. S. et al. Bmprla and Bmpr1b have overlapping functions and are essential for chondrogenesis in vivo. Proceedings of the National Academy of Sciences of the United States of America 102, 5062-5067, doi:10.1073/pnas.0500031102 (2005).

48 Zehentner, B. K., Dony, C. \& Burtscher, H. The transcription factor Sox9 is involved in BMP-2 signaling. Journal of bone and mineral research : the official journal of the American Society for Bone and Mineral Research 14, 1734-1741, doi:10.1359/jbmr.1999.14.10.1734 (1999).

49 Emans, P. J. et al. Autologous engineering of cartilage. Proc Natl Acad Sci U S A 107, 3418-3423, doi:0907774107 [pii]10.1073/pnas.0907774107 (2010). 



\section{Chapter 5}

\section{Inhibition of Cyclooxygenase-2 Decreases Chondrocyte Hypertzophy}

\section{in Endochondral Ossification}

\section{Adapted from:}

\section{Inhibition of Cyclooxygenase-2 Decreases Chondrocyte Hypertrophy in Endochondral Ossification}

Tim J. M. Welting ${ }^{1}$, Marjolein M.J. Caron ${ }^{1}$, Pieter J.Emans ${ }^{1}$, Maarten P.F. Janssen ${ }^{1}$, Kathleen Sanen ${ }^{1}$, Mariëlle M.E. Coolsen ${ }^{1}$, Laura Voss ${ }^{1}$, Don A.M. Surtel ${ }^{1}$, Andy Cremers ${ }^{1}$, Jan-Willem Voncken ${ }^{2}$, and Lodewijk W. van Rhijn ${ }^{1}$.

${ }^{1}$ Department of Orthopaedic Surgery, Maastricht University Medical Center, Maastricht, the Netherlands ${ }^{2}$ Department of Molecular Genetics, Maastricht University Medical Center, Maastricht, the Netherlands European Cells and Materials. (2011); 22:420-436 


\section{Alstract}

Introduction: Skeletogenesis and bone fracture healing involve endochondral ossification, a process during which cartilaginous primordia are gradually replaced by bone tissue. In line with a role for cyclooxygenase-2 (COX-2) in the endochondral ossification process, non-steroidal anti-inflammatory drugs (NSAIDs) were reported to negatively influence bone fracture healing due to impaired osteogenesis. However, the role of COX-2 activity in chondrogenesis has not been addressed before.

Methodology: The role of COX-2 was studied during chondrogenic differentiation of ATDC5 cells and rabbit periosteal explants. BMP-2 was used to increase hypertrophic differentiation and the NSAIDs NS398 and Celecoxib were applied to specifically inhibit COX-2 activity. Chondrogenic outcome was measured by gene- and protein expression analysis, and (immuno)histochemical stainings. For in vivo evidence skeletally immature NZW rabbits were treated with Celecoxib $(10 \mathrm{mg} / \mathrm{kg})$ for 21 days. Growth plate development was analyzed by histochemistry.

Results: Here we show that COX-2 activity fulfils a regulatory role in chondrocyte hypertrophic differentiation. Our data reveal essential cross-talk between COX-2 and bone morphogenic protein-2 (BMP-2) during chondrocyte hypertrophic differentiation. BMP-2 mediated chondrocyte hypertrophy is associated with increased COX-2 expression and pharmacological inhibition of COX-2 activity by NSAIDs (e.g. Celecoxib) decreases hypertrophic differentiation in various chondrogenic models in vitro and in vivo, while leaving early chondrogenic development unaltered. Our findings demonstrate that COX-2 activity is a novel factor partaking in chondrocyte hypertrophy in the context of endochondral ossification.

Conclusions: These observations provide a novel etiological perspective on the adverse effects of NSAIDs on bone fracture healing and may have important implications for the use of NSAIDs during endochondral skeletal development. 


\section{Introduction}

Endochondral ossification is a multistage process that determines the major part of mammalian skeletal development. This process takes place in growth plates of long bones which are populated by highly proliferative chondrocytes, vectorially dictated to differentiate into hypertrophic chondrocytes before dying from apoptosis. The remaining mineralized extra cellular matrix provides a scaffold for infiltrating osteoblasts and osteoclasts to adhere to and remodel, setting the stage for de novo bone deposition ${ }^{1,2}$. Chondrocyte hypertrophic differentiation also plays a role in bone fracture healing which reiterates the endochondral ossification process: initial cartilage callus formation by mesenchymal progenitor cells from the local periosteum and bone marrow, followed by chondrocyte hypertrophy and osteogenic mineralization and remodelling.

Non steroidal anti-inflammatory drugs (NSAIDs) are widely used systemic inhibitors of inflammatory prostaglandin production by cyclooxygenases (COX-1 and COX-2 $)^{3}$. In orthopaedic surgery the use of NSAIDs is under debate, as systemic NSAID treatment delays spinal fusion in patients and animal models ${ }^{4}$ and fracture healing of the long bones ${ }^{5}$ ${ }^{-8}$. This property of NSAIDs is, however, exploited in the clinic to prevent periprosthetic heterotopic calcification after hip surgery ${ }^{9}$. The action of NSAIDs has been studied in the context of osteogenesis, and several observations link prostaglandin $\mathrm{E}_{2}\left(\mathrm{PGE}_{2}\right)$ and $\mathrm{COX}-2$ to osteoblast differentiation ${ }^{10-13}$. Mice lacking the prostaglandin synthesizing enzymes COX-2 or microsomal prostaglandin E synthase-1 (mPGES-1) show impaired fracture healing caused by insufficient $\mathrm{PGE}_{2}$ synthesis ${ }^{13,14}$. Expression of bone morphogenic protein-2 (BMP-2) is regulated by COX-2 activity ${ }^{10}$ and downstream signalling of BMP-2 in osteogenesis involves formation of a runt-related transcription factor 2 (Runx2) - Smad transcription factor complex ${ }^{15}$. Combined these data provide insight into the role of COX-2 activity during osteogenesis and suggest a plausible explanation for the adverse effects of NSAIDs on bone healing.

Recently, prostaglandins were shown to influence chondrogenesis in vitro ${ }^{16,17}$. Prostaglandin receptors and COX-enzymes localize to the developing growth plate ${ }^{18,19}$ and BMP-2, whose expression depends on COX-2 activity ${ }^{10}$, is described to determine endochondral ossification by regulating chondrocyte differentiation ${ }^{20}$. Hypertrophic chondrocytes uniquely express the collagen type $\mathrm{X}$ alpha- 1 chain $(\mathrm{Col10} 1)^{21}$ whose expression depends, amongst others, on (BMP-2 induced) Runx2/core-binding factor subunit alpha-1 (Cbfa1) ${ }^{22}$. 
However, the exact description of a role for COX-2 in chondrogenesis is lacking, and the functional relation between BMP-2, COX-2 and chondrocyte hypertrophic differentiation in the context of endochondral ossification remains unclear at present. We therefore hypothesized that chondrocyte hypertrophic differentiation is determined by BMP-2 via a COX-2 dependent mechanism. The hypothesis was addressed using different models for endochondral ossification. Our results reveal that inhibition of COX-2 activity specifically decreases the level of BMP-2 induced hypertrophic differentiation in endochondral culture models, and systemic inhibition of COX-2 activity in vivo resulted in significantly impaired chondrocyte hypertrophic differentiation in rabbit growth plates.

\section{Materials and Methods}

\section{$\underline{2.1 \text { ATDC5 cell culture and chondrogenic differentiation }}$}

ATDC5 cells were cultured in proliferation medium (DMEM/F12 (Invitrogen, Carlsbad, CA, USA), 5\% FCS (PAA, Pasching, Austria), 1\% NEAA (Invitrogen) and 1\% antibiotic/ antimycotic (Invitrogen) $)^{23}$. Cells were differentiated in differentiation medium (proliferation medium supplemented with $10 \mathrm{mg} / \mathrm{ml}$ Insulin (Sigma, St. Louis, MO, USA), $10 \mathrm{mg} / \mathrm{ml}$ Transferrin (Roche Applied Science, Woerden, the Netherlands) and $30 \mathrm{nM}$ Sodium Selenite (Sigma)). The day prior to differentiation, ATDC5 cells were plated at 6400 cells $/ \mathrm{cm}^{2}$ in culture dishes and allowed to adhere overnight in proliferation medium. Chondrogenesis was initiated by changing the medium to differentiation medium. Differentiation medium was strictly changed at day 2, 4, 6 and 8 and from day 8 every day. When indicated BMP-2 was used (Wyeth). COX-2 activity was inhibited by NS398 (Cayman Chemicals, Ann Arbor, MI, USA) or Celecoxib (LC Laboratories, Woburn, MA, USA). Phosphorylation of Smad1/5/8 was inhibited by Dorsomorphin (Santa Cruz Biotechnologies, CA, USA)

\subsection{Periosteum Agarose Culture (PAC) chondrogenic differentiation}

Periosteum was harvested from the proximal tibia of skeletally mature New Zealand white (NZW) rabbits. Post-mortem control animals were freshly obtained from an unrelated study, no ethical approval was necessary. Periosteum was harvested from these rabbits within 15 minutes after sacrifice. Presence of the cambium layer was checked by 
histology. The periosteum was transported in HEPES buffered DMEM/F12 supplemented with antibiotics. Per rabbit the harvested Periosteum (generally $5 \times 5 \mathrm{~mm}$ ) was prepared as a $3 \times 3 \mathrm{~mm}$ piece using a sterile surgical blade and embedded in 24-wells plates using the following procedure adapted from O'Driscoll et al. ${ }^{24}: 250 \mathrm{ml} 1 \%$ high melting agarose $/ 0.9 \% \mathrm{NaCl}\left(37^{\circ} \mathrm{C}\right)$ was applied on the bottom of the well and allowed to solidify. Periosteum pieces were put on top and allowed to float in $375 \mathrm{ml} 1 \%$ low melting agarose $/ 0.9 \% \mathrm{NaCl}\left(37^{\circ} \mathrm{C}\right)$. After solidifying, an additional $375 \mathrm{ml} 1 \%$ low melting agarose $/ 0.9 \% \mathrm{NaCl}$ was applied. The agarose was allowed to solidify for an additional ten minutes. One milliliter of PAC proliferation medium (DMEM/F12, 10\% FCS, 1\% P/S, 1\% NEAA) was added and incubated overnight at $37^{\circ} \mathrm{C} / 5 \% \mathrm{CO}_{2}$. After overnight incubation, medium was changed to PAC differentiation medium (proliferation medium supplemented with 1\% ITS (insulin-transferrin- sodium selenite media supplement, Invitrogen), $50 \mathrm{mg} /$ $\mathrm{ml}_{\mathrm{L}}$-ascorbic acid-2-phosphate (Sigma), $10 \mathrm{ng} / \mathrm{ml}$ transforming growth factor $\beta 3$ (TGF-b3) (R\&D, Minneapolis, MN, USA)). PAC's were maintained in differentiation medium by changing medium every three days.

\subsection{Immunoblotting}

After washing with $0.9 \% \mathrm{NaCl}$, ATDC5 cells were lysed in RIPA buffer $(150 \mathrm{mM} \mathrm{NaCl}$, $1 \%$ NP-40, 0.5\% sodium dexoycholate, $0.1 \%$ SDS, $50 \mathrm{mM}$ Tris-HCl $\mathrm{pH} 8.00,5 \mathrm{mM}$ EDTA, $0.5 \mathrm{mM}$ dithiotreitol, $2 \mathrm{mM}$ phenylmethylsulfonylfluoride) and homogenized on ice by sonication (Soniprep 150, MSE). Insoluble material was removed by centrifugation ( 5 minutes; $13,000 \mathrm{x} \mathrm{g} ; 4^{\circ} \mathrm{C}$ ). Total protein concentration was determined using a BCA (bicinchoninic acid) assay (Sigma). Polypeptides were separated by SDS-PAGE and transferred to nitrocellulose membranes by electroblotting. Immunodetection of Col2a1, Col10a1, GAPDH (glyceraldehyde 3-phosphate dehydrogenase), Runx2, pSmad1/5/8, $\beta$-catenin and COX-2 was performed using polyclonal goat and rabbit sera (Col2a1 (SouthernBiotech, Birmingham, AL, USA), Col10a1 (Calbiochem, Darmstadt, Germany), COX-2 (Oxford Biomedical Research, Oxford, UK), pSmad1/5/8 (Cell Signaling Technology, Boston, MA, USA) and mouse monoclonal antibodies recognizing GAPDH (Fitzgerald, Acton, MA, USA), $\beta$-catenin (BD Biosciences, Breda, the Netherlands) and Runx2 (MBL, Woburn, MA, USA). Horseradish peroxidase (HRP)-conjugated immunoglobulins were purchased from DakoCytomation (Glostrup, Denmark) and applied as secondary antibody. Bound antibodies were visualized by enhanced chemiluminescence detection procedures. Several housekeepers were used to verify total protein loading. 
However, although equally loaded (quantitatively determined by BCA assay and visually by Ponseau-S (on membrane) and Coomassie Brilliant Blue staining (in gel)), GAPDH, $\alpha$-tubulin and b-actin protein expression appeared to be regulated during the course of chondrogenesis. However, these protein housekeepers were stable amongst different samples from the same chondrogenic differentiation stage (e.g. day 14).

\subsection{Real time quantitative PCR (RT-qPCR)}

To isolate total RNA from PAC cultures, tissue was cut in pieces and soaked overnight in $500 \mathrm{ml}$ Trizol (Invitrogen, Breda) at $4^{\circ} \mathrm{C}$. For RNA isolation from ATDC5 cells, cells were washed with $0.9 \% \mathrm{NaCl}$ and disrupted with $500 \mathrm{ml}$ Trizol. Total RNA from the aqueous phase was precipitated by using isopropanol and centrifugation (13.000 x g; 30 minutes; $4^{\circ} \mathrm{C}$ ). Pellets were washed with $80 \%$ ethanol, allowed to airdry and dissolved in DEPCtreated water. Genomic DNA was removed by DNase I (Roche) treatment (20 U; 1 hour; $37^{\circ} \mathrm{C}$ ). RNA was precipitated with NaAc/ethanol and subsequent centrifugation. Pellets were washed with $80 \%$ ethanol and allowed to airdry. Purified RNA was dissolved in 20 $\mathrm{ml}$ DEPC-treated water and analyzed spectrophotometrically (Nanodrop, Thermo Scientific, the Netherlands). For cDNA synthesis, $200 \mathrm{ng}$ total RNA was used in a $25 \mathrm{ml}$ standard RT-reaction containing 1x RT-buffer (Promega, Madison, MA, USA); 2 ng/ml random primer (Promega); $0.5 \mathrm{mM}$ per dNTP (Eurogentec, Seraing, Belgium); $10 \mathrm{mM}$ dithiotreitol; $0.4 \mathrm{U} / \mathrm{ml}$ RNasin (Promega); $4 \mathrm{U} / \mathrm{ml}$ M-MLV reverse transcriptase (Promega).

Real time qPCR was performed in triplicate with Mesagreen qPCR mastermix plus for SYBR ${ }^{\circledR}$ Green (Eurogentec) in 96-well optical plates according to the manufacturer's protocol. Forward and reverse primers were used at $300 \mathrm{nM}$ each. Gene expression was determined using an ABI PRISM ${ }^{\circledR} 7700$ Sequence Detection System (Applied Biosystems, Foster City, CA, USA). Serially diluted standard curves were included to quantify the samples. Samples were heated for 10 minutes at $95^{\circ} \mathrm{C}$ and DNA was amplified by 40 cycles of 15 seconds $95^{\circ} \mathrm{C}$ and 1 minute $60^{\circ} \mathrm{C}$, followed by a melting curve. Data were analyzed using Sequence Detection Software version 1.7 (Applied Biosystems, Foster City, CA, USA) using the standard curve method. Values were normalized to $\beta$-actin (in contrast to $\beta$-actin protein levels, $\beta$-actin mRNA levels are stable during chondrogenesis). The primer sequences for detection of Col2a1, Col10a1, $\beta$-actin, BMP-2, Runx2, Mef2c, MMP13, VEGF-A, COX-2 and ALP were based on the coding sequences of the respective mRNAs. The sequences are listed in Table 5.1. Values were normalized 
to $\beta$-actin mRNA levels and fold increase gene expression was calculated relatively to the mean expression value at day 0 in differentiation.

\section{$\underline{2.5 P G E_{2} \text { measurement }}$}

Inhibition of COX-2 activity was determined by measuring prostaglandin $\mathrm{E}_{2}\left(\mathrm{PGE}_{2}\right)$ in culture medium or blood plasma. $\mathrm{PGE}_{2}$ concentration was determined by a standardized EIA according to the manufacturers' protocol (Cayman Chemical).

Table 5.1: Primer sequences for RTqPCR

\begin{tabular}{lll}
\hline Oligo sets mouse & $\underline{\text { Forward }}$ & $\underline{\text { Reverse }}$ \\
\hline Col2a1 & '5-TGGGTGTTCTATTTATTTATTGTCTTCCT-3' & '5-GCGTTGGACTCACACCAGTTAGT-3' \\
Col10a1 & '5-CATGCCTGATGGCTTCATAAA-3' & '5-AAGCAGACACGGGCATACCT-3' \\
Runx2 & '5-CGATGAAGACCCCAACCCTAA-3' & '5-ACTGGTAATGGCATCAAGGGATA-3' \\
COX-1 & '5-TCCTCACAGTGCGGTCCAA-3' & '5-AAGGCCTCCCAGCTGATGTAG-3' \\
COX-2 & '5-GGCCATGGAGTGGACTTAAA-3' & '5-AAGTGGTAACCGCTCAGGTG-3' \\
BMP-2 & '5-AGAAAAGCGTCAAGCGAAACA-3' & '5-GTCCACGTACAAAGGGTGTCTCT-3' \\
Mef2c & '5-GGGCCTCAATGGCTGTGA-3' & '5-CTCAGACTCAGGGCTGTGACCTA-3' \\
Mmp13 & '5-CGATGAAGACCCCAACCCTAA-3' & '5-ACTGGTAATGGCATCAAGGGATA-3' \\
VEGF-A & '5-GTCAGAGAGCAACATCACCA-3' & '5-CATCTGCTGTGCTGTAGGAA-3' \\
Alp & '5-CCGATGGCACACCTGCTT-3' & '5-GGAGGCATACGCCATCACAT-3' \\
$\beta$-Actin & '5-GACAGGATGCAGAAGGAGATTACTG-3' & '5-CCACCGATCCACACAGAGTACTT-3' \\
\hline Oligo sets rabbit & $\underline{\text { Forward }}$ & $\underline{\text { Reverse }}$ \\
\hline Col2a1 & '5-TGGGTGTTCTATTTATTTATTGTCTTCCT-3' & '5-GCGTTGGACTCACACCAGTTAGT-3' \\
Col10a1 & '5-AACCTGGACAACAGGGACTTACA-3' & '5-CCATATCCTGTTTCCCCTTCTG-3' \\
COX-2 & '5-GGCCATGGAGTGGACTTAAA-3' & '5-AAGTGGTAACCGCTCAGGTG-3' \\
$\beta$-Actin & '5-GACAGGATGCAGAAGGAGATTACTG-3' & '5-CCACCGATCCACACAGAGTACTT-3'
\end{tabular}

The 5' to 3' forward and reverse oligonucleotide sequences used for RT-qPCR are listed in the table.

\subsection{Alkaline Phosphatase activity}

Enzymatic activity of Alkaline Phosphatase (Alp) in ATDC5 cultures was determined using a colorimetric assay. Cells were lysed in freshly prepared collection buffer (1.5 M Tris$\mathrm{HCl} \mathrm{pH} \mathrm{9.0;} \mathrm{2 \%} \mathrm{(v/v)} \mathrm{Triton} \mathrm{X-100)} \mathrm{and} \mathrm{homogenized} \mathrm{on} \mathrm{ice} \mathrm{by} \mathrm{sonication} \mathrm{(Soniprep} \mathrm{150,}$ MSE). Insoluble material was removed by centrifugation ( 5 minutes; $13,000 \mathrm{x} \mathrm{g} ; 4^{\circ} \mathrm{C}$ ). Total protein concentration was determined using a BCA assay (Sigma). In flat bottom 96 wells plates, containing assay buffer (1.5 M Tris-HCl pH 9.0; $1 \mathrm{mM} \mathrm{ZnCl}_{2} ; 1 \mathrm{mM} \mathrm{MgCl}$; $7.5 \mathrm{mM}$ p-nitrophenyl phosphate), Alp activity was determined by measuring Alp-depend enzymatic conversion of p-nitrophenyl phosphate to nitrophenyl phosphate by spectrophotometrical analyses at $405 \mathrm{~nm}$. A calibration curve containing an increasing 
concentration of nitrophenyl phosphate was used to determine the absolute amount of Alpgenerated nitrophenyl phosphate in time. Values were normalized to total protein concentration and expressed as Units Alp activity $(1 \mathrm{U}=1 \mu \mathrm{mol}$ nitrophenyl phosphate/ minute/gram).

\subsection{Cell proliferation}

Growth curves for ATDC5 cells were generated by determining DNA content using crystal -violet. Cells were fixed with $4 \%$ paraformaldehyde in phosphate buffered saline for ten minutes at room temperature. After washing, cells were air dried for further analysis. Fixed cells were incubated with $0.1 \%$ crystal-violet for 30 minutes at room temperature. Cells were washed with water to remove excess crystal-violet and allowed to air-dry in the dark overnight. Crystal-violet was extracted by incubation with $10 \%$ acetic acid for 15 minutes under continuous agitation. Extracted crystal-violet was determined spectrophotometrically at $590 \mathrm{~nm}$ using a plate reader (Biorad, the Netherlands).

\section{$\underline{2.8 \text { (Immuno)histochemistry }}$}

PAC tissue samples or mouse growth plates were decalcified in formalin/EDTA, dehydrated following standard procedures and embedded in paraffin. Tissue sections were cut at $5 \mathrm{~mm}$ and positioned on Superfrost Plus slides (Menzel, Germany). Before histochemistry, tissue sections on slides were deparafinized and rehydrated using standard protocols. Proteoglycans were stained with Safranin-O (0.1\%) and counterstained with Fast Green (0.1\%). Stained sections were dehydrated and mounted in Histomount (Thermo Shandon) for microscopic analysis.

For immunohistochemical analysis of Runx2, Col2a1, Col10a1 and Alp in PAC sections, rehydrated sections were treated with $0.4 \%$ hyaluronidase (Col2al and Col10a1 only). Endogenous peroxidase activity was inactivated by Peroxidase-Blocking solution (Dako, REAL) and samples were blocked with 5\% skim milk (Runx2), 10\% normal sheep serum (Col2a1 and Col10a1) or 10\% normal rabbit serum (Alp) for 30 minutes. Anti-Cbfa1/ Runx2 monoclonal antibody (MBL) was used at 1:700. Mouse monoclonal anti-Col2a1 (II-II6B3; Developmental Studies Hybridoma Bank) and mouse negative control IgG1 (Dako) were used at 1:50 and same IgG1 concentration, respectively. Rabbit polyclonal anti-Col10a1 and negative control non-immune rabbit serum were used at 1:1000. Goat polyclonal affinity purified anti-Alp IgG (R\&D systems) and negative control non-immune goat total IgG (protein-G purified) were used at 1:50 and same IgG concentration, respec- 
tively. Primary antibodies were incubated for one hour at room temperature. Unbound antibodies were removed by washing with TBS-T. For Runx2 detection sheep-anti-mousebiotin (Amersham Biosciences) was used as secondary antibody at 1:200 and incubated for one hour at room temperature in TBS/BSA. Sections were washed with TBS-T and Biotin conjugate was detected with SA-HRP (Beckman Coulter) at 1:500 in TBS/BSA and DAB chromogen (Dako). For Col2al and Col10a1 bound antibodies were visualized with HRP labelled anti-rabbit or anti-mouse secondary antibodies (Dako, EnVision+ System-HRP labelled Polymer) by incubation for 30 minutes at room temperature. Unbound antibodies were removed by rinsing in PBS-T. For Alp detection bound primary antibodies were detected by 1:500 diluted HRP labelled rabbit-anti-goat secondary antibodies (Dako). For detection, DAB chromogen substrate (Dako) was used. Stained sections were mounted in Histomount as described above. In mouse growth plates from 6 week old mice (Maastricht University Medical Center animal ethical committee approval ID: DEC 2008-042) COX-2 was detected using citrate-mediated antigen retrieval and 1:50 anti-COX-2 monoclonal antibody (BD Biosciences). Mouse negative control IgG1 (Dako) was used at same concentration. Detection of COL10A1 and ALP in these growth plates was carried out as described above for the PAC sections. Visualisation of anti-COX-2, anti-ol10a1 and antiAlp primary antibodies was performed as described above.

\subsection{Celecoxib treatment of rabbits and analysis of growth plates}

The experiment was approved by the Maastricht University animal ethical committee (DEC 2010-027-g). celecoxib (10 mg/kg) (Pfizer) was orally administered to skeletally immature New Zealand white rabbits (107 days old) for 21 days on a daily basis in $1 \mathrm{ml}$ Critical Care-paste (Oxbow Animal Health). Control animals received $1 \mathrm{ml}$ paste only. The groups consisted of six control animals and seven celecoxib-treated animals. Rabbits were euthanized after three weeks by an overdosis of pentobarbital. The left ulnas were isolated and fixed in formalin. Growth plates were decalcified in $0.5 \mathrm{M}$ EDTA $\mathrm{pH} 7.8$ for three weeks. After that growth plates were briefly (20 hours) incubated in 1:5 diluted TBD-1 Rapid Decalcifier (Thermo Shandon). Growth plates were cut in two halfs perpendicular to the growth plate plane, dehydrated and embedded in paraffin. From the anterior half, $5 \mathrm{~mm}$ sections were cut. Sections where stained with Haematoxylin and further processed for microscopic analysis using a Zeiss Axioscope A.1 (with AxioVision 4.8 software). The anatomical middle of the growth plates was used as an internal reference point. From each growth plate three consecutive sections were measured at both sides at half the distance 
from the anatomical reference point. At this point a standardized box with a width of 250 $\mathrm{mm}$ was defined that fitted an average number of 10 chondrocyte columns. Within the box the lengths of the proliferative and hypertrophic zones were determined by three independent blinded observers.

\subsection{Statistics}

Statistical analyses on the rabbit growth plates were performed as follows. Measurements from consecutive sections and both sides of the same growth plate were averaged. Data of the control (six rabbits) and celecoxib (seven rabbits) groups were tested for statistical significance using SPSS 16.0 software. Normal distribution of the data was confirmed by a Shapiro-Wilk normality test and subsequently the significance of the total length of the growth plates, length of the proliferative zone, length of the hypertrophic zone and the ratio between the proliferative and hypertrophic zone were determined using MannWhitney tests. A $p<0.05$ was defined as significant. Next to the above statistics, other statistical calculations were determined by unpaired two-tailed Student's t-tests using Graphpad PRISM 5.0. A $\mathrm{p}<0.05$ was defined as significant. To test for normal distribution of the input data, D'Agostino-Pearson omnibus normality tests were performed. All herein presented quantitative data sets passed the normality tests.

\section{Results}

\section{$\underline{3.1 \text { BMP-2 increases COX-2 expression during endochondral ossification }}$}

COX-2 expression was determined in the ATDC5 model. ATDC5 differentiation follows a well-defined endochondral program from undifferentiated chondroprogenitor to hypertrophic chondrocyte. Differentiation was initiated as described previously ${ }^{23}$ and was evident after 7 days of differentiation as measured by Collagen type II (Col2a1) mRNA expression. Col2a1 expression stabilized 10 days after induction of differentiation (Figure 5.1A). Hypertrophic differentiation is characterized by upregulation of Col10a1 mRNA from 10 days in differentiation (Figure 5.1A) and increased further at 14 days and later time points (see also below; Figure 5.5). As an additional hypertrophic marker, increased expression of alkaline phosphatase (Alp) mRNA was confirmed (Figure 5.1A). Similarly, increased mRNA expression was observed over time for other hypertrophic markers as 
A

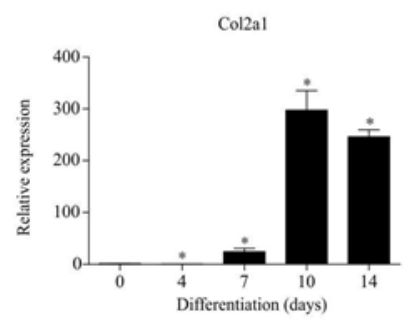

Alp

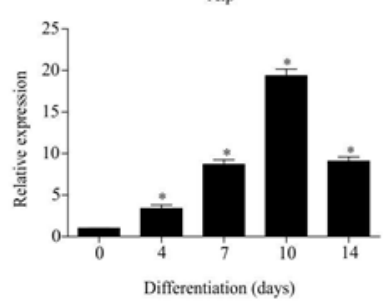

C

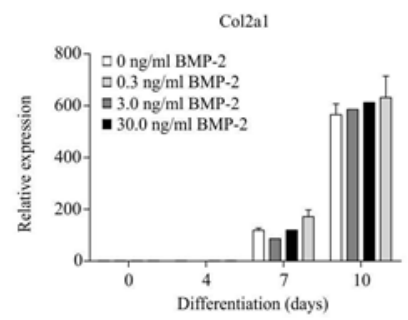

Alp

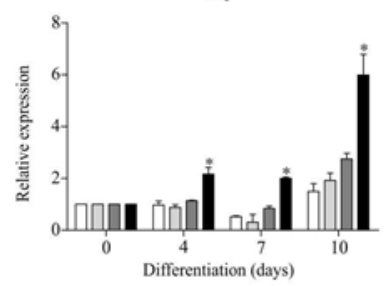

E

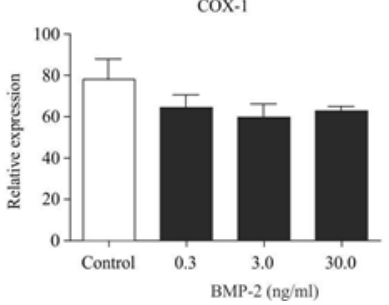

B

Colloal

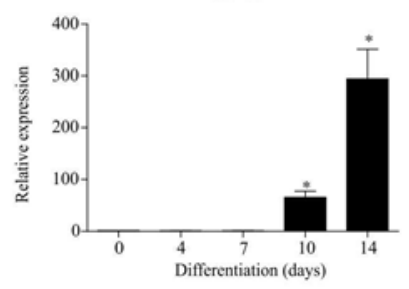

cox-2

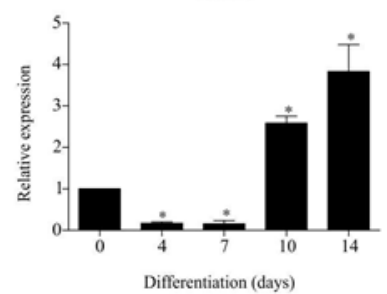

D
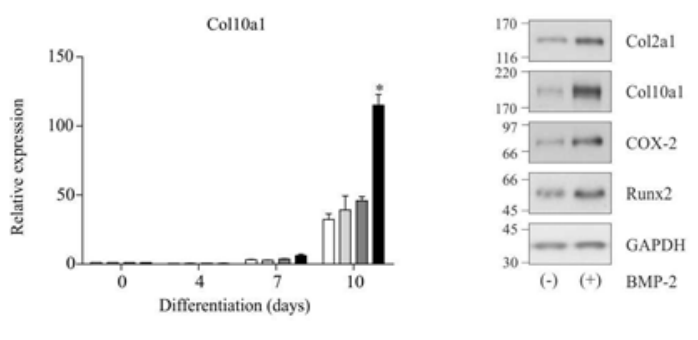

COX-2

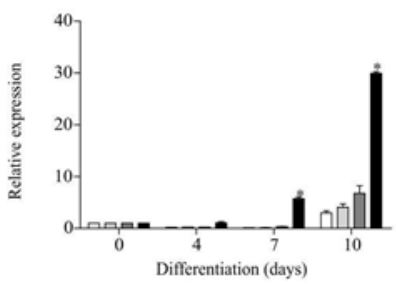

$\operatorname{cox}-2$

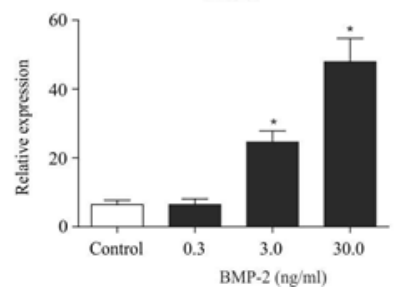



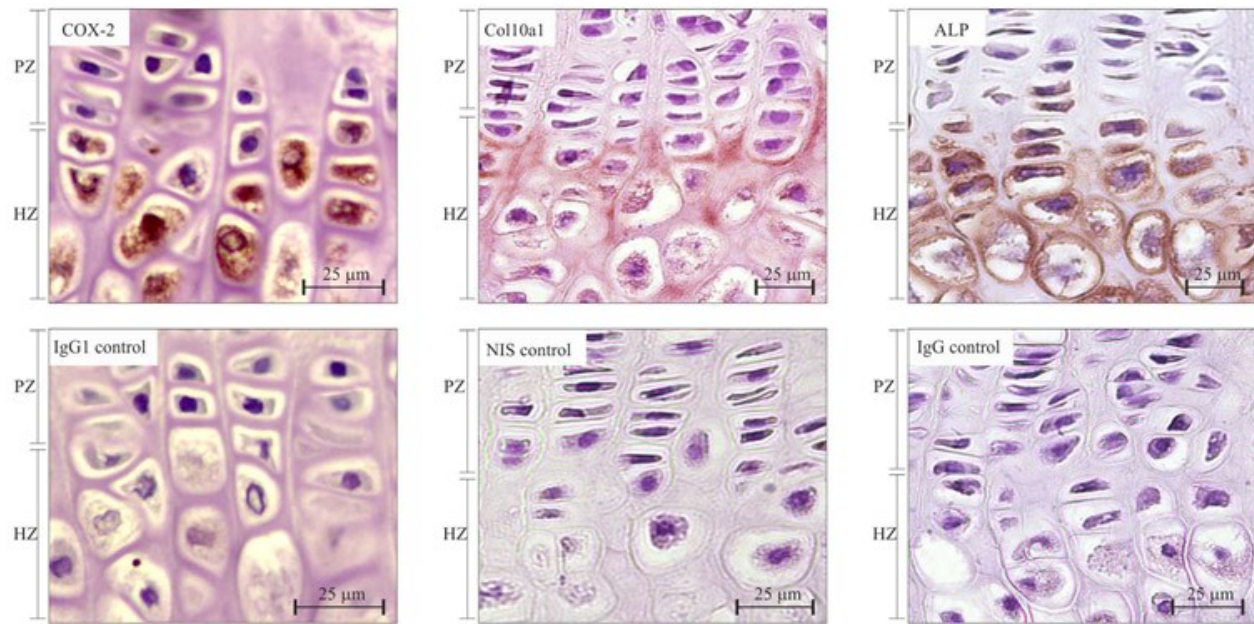

Figure 5.1: $C O X-2$ expression and BMP-2 responsiveness during chondrogenic differentiation

Differentiated ATDC5 cells were harvested at day 0, 4, 7, 10 or 14. (A). Induction of Col2a1, Col10a1, Alp and COX-2 mRNA expression was determined by RT-qPCR. Error bars represent mean \pm s.d.. (B). Col2a1, Col10a1 and COX-2 protein expression at 0, 7 and 14 days after initiation of differentiation. GAPDH was used as housekeeper. Molecular weight markers $(\mathrm{kDa})$ are depicted on the right. $(C)$. BMP-2 was added to differentiation medium at $0 \mathrm{ng} / \mathrm{ml}$ (white bars), $0.3 \mathrm{ng} / \mathrm{ml}$ (light grey bars), $3.0 \mathrm{ng} / \mathrm{ml}$ (dark grey bars) or $30 \mathrm{ng} / \mathrm{ml}$ (black bars). Induction of Col2a1, Col10a1, Alp and COX-2 mRNA expression was determined at day 0, 4, 7 and 10 by RTqPCR. Sample size is 9 for every time point. (D). Protein samples from 0 and $30 \mathrm{ng} / \mathrm{ml} \mathrm{BMP-2}$ conditions (day 14) were analyzed for Col2a1, Col10a1, COX-2, Runx 2 and GAPDH by immunoblotting. (E). ATDC5 cells were differentiated in the presence of increasing BMP-2 concentrations and induction of COX-1 and COX-2 mRNAs at day 14 was determined by RT-qPCR. Sample size is 9 for every BMP-2 concentration. $(F)$. Spatiotemporal expression of COX-2, Col10a1 and ALP in 6 weeks old mouse growth plates was determined by immunohistochemistry (IHC). Left upper panel: anti-COX-2, left lower panel: IgG1-control; middle upper panel: antiCol10a1, middle lower panel: non-immune serum (NIS) control; right upper panel: anti-ALP, right lower panel: IgG control. $\mathrm{PZ}=$ proliferative zone, $\mathrm{HZ}=$ hypertrophic zone. Scale bars indicate $25 \mu \mathrm{m}$. * indicates $\mathrm{p}<0.05$.

Runx2, myocyte-specific enhancer 2c (Mef2c), matrix metalloproteinase 13 (Mmp13) and vascular endothelial growth factor a (VEGF-A) (see below; Figure 5.5). Col2a1 and Col10a1 protein expression followed similar expression patterns as their respective mRNAs (Figure 5.1B). COX-2 mRNA expression was almost 4-fold upregulated at 14 days in differentiation (Figure 5.1A) and immunoblotting showed that COX-2 protein is expressed during chondrogenesis from day 7 onward (Figure 5.1B). We next determined whether in chondroprogenitor cells COX-2 expression is regulated by BMP-2. In a BMP-2 dose-response experiment COX-2 mRNA expression responded significantly to a concentration of $30 \mathrm{ng} / \mathrm{ml}$ BMP-2 and was accompanied by a significantly increased Col10a1 and 
ALP mRNA expression (Figure 5.1C). Although Col2al mRNA expression was only marginally affected, $30 \mathrm{ng} / \mathrm{ml}$ BMP-2 did result in higher Col2a1 protein expression, as well as increased expression of Runx2 and Col10a1 14 days in differentiation (Figure 5.1D). Also COX-2 protein expression responded positively to this BMP-2 condition (Figure 5.1D). BMP-2 specifically provoked COX-2 expression, as COX-1 expression remained unaltered under various BMP-2 concentrations (Figure 5.1E). To further corroborate the relation between $\mathrm{COX}-2$ and chondrocyte hypertrophic differentiation, spatio-temporal expression of COX-2 was determined in the murine growth plate. COX-2 is specifically expressed in hypertrophic chondrocytes that express Col10a1 and Alp (Figure 5.1F). Throughout hypertrophic differentiation (i.e. more distal to the proliferative zone), expression of COX-2 increased. These data demonstrate that expression of COX-2 correlates with chondrocyte hypertrophic differentiation and suggest that BMP-2 plays a central role in this process.

\subsection{COX-2 activity is required for chondrocyte hypertrophic differentiation}

To examine COX-2 activity in the context of chondrocyte hypertrophic differentiation, we analyzed enzymatic activity of COX-2 by measuring $\mathrm{PGE}_{2}$ levels in culture supernatants. $\mathrm{PGE}_{2}$ was readily detectable at 10 days in differentiation and further increased at 14 days (Figure 5.2; white bars; (-) BMP-2), in good agreement with increased COX-2 expression (Figure 5.1A/B). In line with the observed BMP-2 responsiveness of COX-2 expression (Figure 5.1C-E), increased $\mathrm{PGE}_{2}$ synthesis in the presence of $30 \mathrm{ng} / \mathrm{ml} \mathrm{BMP-2} \mathrm{(Figure} \mathrm{5.2;}$ white bars; (+) BMP-2) indicated enhanced levels of enzymatically active COX-2. To substantiate a functional link between COX-2 protein levels and prostaglandin synthesis, we pharmacologically inhibited COX-2 enzyme activity using NS398. Specificity of NS398 for COX-2 over COX-1 was confirmed (Figure 5.3). Addition of NS398 almost completely abolished $\mathrm{PGE}_{2}$ synthesis to near-background levels (Figure 5.2; grey and black bars), confirming a role for COX-2-mediated prostaglandin synthesis during chondrogenic differentiation.

Next we analysed whether COX-2 inhibition influences chondrogenic differentiation in the absence or presence of exogenous BMP-2. Differentiation medium was supplemented with increasing NS398 concentrations and chondrogenic marker expression was analyzed at 14 days into differentiation. In the absence of BMP-2, $20 \mu \mathrm{M}$ NS398 reduced Col10a1 protein and mRNA expression (Figure 5.4A/B). In line with reduced Col10a1 expression, Runx2 protein expression was reduced by $20 \mu \mathrm{M}$ NS398 (Figure 5.4A), whereas under all NS398 


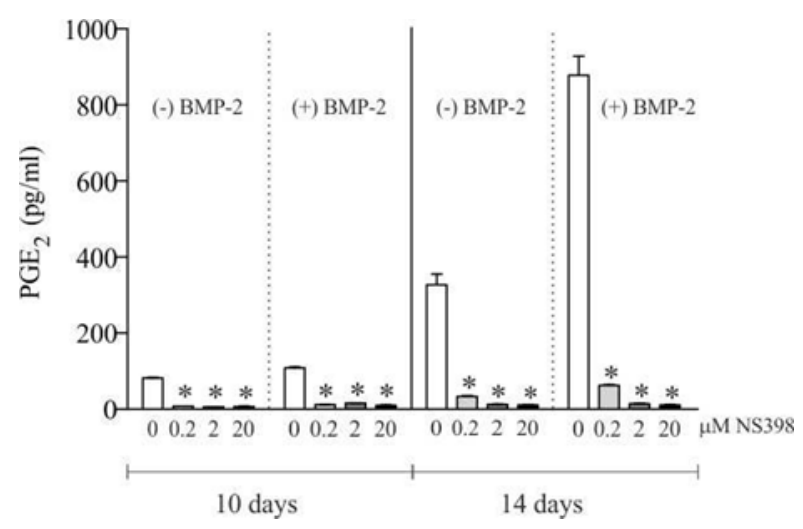

Figure 5.2: Inhibition of COX-2 activity during ATDC5 differentiation

ATDC5 cells were differentiated in the absence or presence of $30 \mathrm{ng} / \mathrm{ml} \mathrm{BMP-2}$ and with increasing amounts of NS398 (black bars). COX-2 activity was determined by measuring PGE $_{2}$ synthesis (pg/ml) using an EIA. Absolute values are shown. Bars represent the mean $\mathrm{PGE}_{2}$-values of independent triplicate samples of 3 individual experiments ( 9 independent supernatant samples) and error bars represent mean \pm s.d.. $*$ indicates $\mathrm{p}<0.05$.

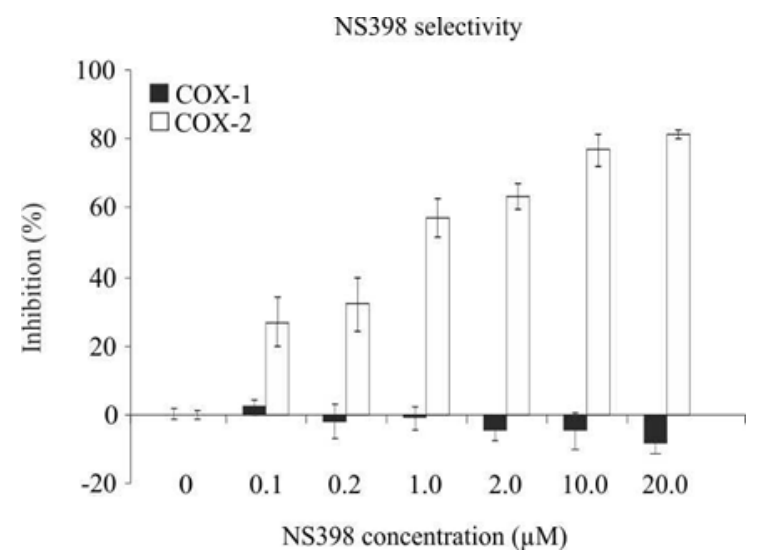

Figure 5.3: Selectivity of NS398 for COX-2

The selectivity of NS398 for COX-2 over COX-1 was determined using a COX-1/COX-2 colorimetric inhibition assay. NS398 was tested at $0,0.1,0.2,1.0,2.0,10.0$ and $20.0 \mu \mathrm{M}$. The inhibitory capacity was determined as "percent inhibition" compared to a maximum activity control condition. Bars represent average inhibition percentages of COX-1 (black bars) and COX-2 (white bars). Measurements were done in triplicate and error bars represent the variances in inhibitory activity. 
conditions Col2a1 protein and mRNA levels remained unaltered (Figure 5.4A/B). In the presence of exogenous BMP-2 (30 ng/ml), overall Col2a1, Col10a1, Alp and Runx2 levels were increased, confirming its pro-chondrogenic action (Figure 5.4A/B). The exposure to BMP-2 evoked an increased NS398 responsiveness, as Col10a1, Alp and Runx2 expression are more efficiently downregulated by NS398 as compared to the condition without BMP-2 (Figure 5.4A/B), whereas Col2al protein and mRNA expression remained unchanged with increasing NS398 concentrations under BMP-2 conditions (Figure 5.4A/B).

Reduced endogenous BMP-2 mRNA levels under BMP-2 supplemented conditions point to a negative auto-feedback mechanism (Figure 5.4C). In addition, the sharp NS398 dosedependent decline of BMP-2 mRNA levels suggests a crucial COX-2 activity-dependent positive effect on endogenous BMP-2 regulation (Figure 5.4C). The negative effect of COX-2 inhibition on chondrocyte hypertrophic differentiation was further established by measuring Alp enzymatic activity (Figure 5.4D). Following ATDC5 differentiation, Alp

A

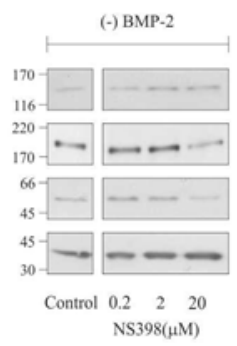

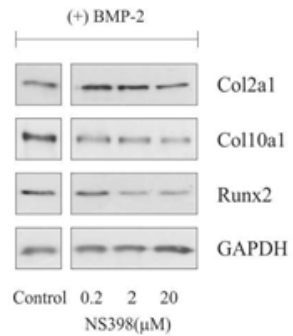

D

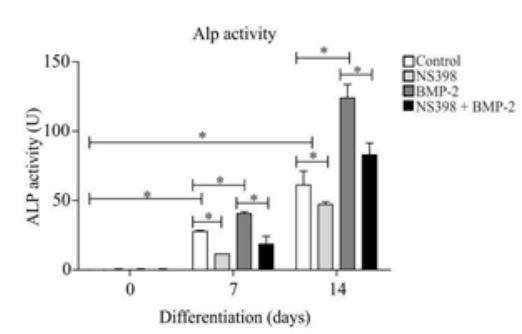

B

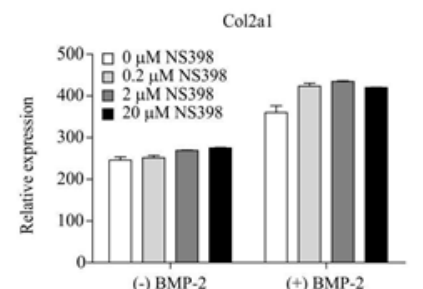

(-) BMP-2

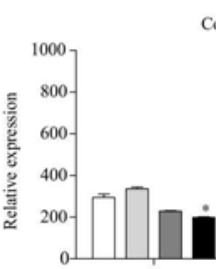

(-)BMP-2

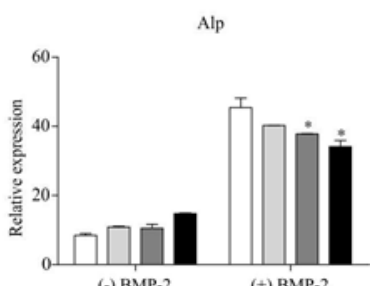

(-) BMP-2

(+) BMP-2

E

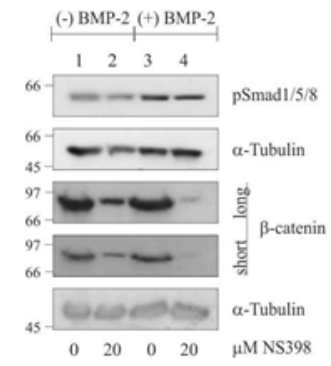

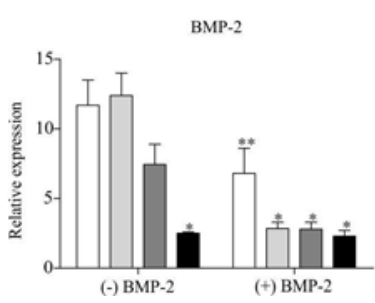

$\underline{139}$ 
Figure 5.4: Inhibition of COX-2 activity decreases chondrocyte hypertrophic differentiation

(A). Increasing NS398 concentrations were added to differentiating ATDC5 cultures in the absence (left panel set) or presence of $30 \mathrm{ng} / \mathrm{ml}$ BMP-2 (right panel set). Protein expression of Col2a1, Col10a1 and Runx2 was analysed in day 14 cell extracts. (B). Induction of Col2a1, Col10a1 and Alp mRNA expression in day 14 samples from same experiment as (A). was determined. White bars: control, grey and black bars: NS398 conditions. (C). COX2 and BMP-2 mRNA induction was determined in similar samples as (B). In addition to shown statistical analyses, for BMP-2 mRNA the statistical significance was also determined between the (-) BMP-2 and (+) BMP-2 condition without NS398 (** indicates $\mathrm{p}<0.05$ ). (D). ATDC5 cells were differentiated for 7 or 14 days in the absence or presence of BMP-2 $(30 \mathrm{ng} / \mathrm{ml})$ or NS398 $(20 \mu \mathrm{M})$ and combinations thereof. Alp enzymatic activity $(\mathrm{U})$ of triplicate samples was determined and normalized to total protein content. $(E)$. In cell extracts from A., levels of p-Smad1/5/8 (upper panel set) and b-catenin (lower panel set; long and short refer to different ECL exposure times) were determined. * indicates $\mathrm{p}<0.05$.

activity increased and consistent with above findings the presence of $30 \mathrm{ng} / \mathrm{ml} \mathrm{BMP-2}$ evoked significantly higher Alp activity at 7 and 14 days in differentiation. In agreement with the anti-hypertrophic action of COX-2 inhibition, Alp activity was significantly decreased in NS398 conditions. Consistent with a positive feedback mechanism, BMP-2 supplementation increased COX-2 expression (Figure 5.1C-E and Figure 5.4C). Canonical Wnt- and Smad-signalling are important regulators of chondrocyte hypertrophic differentiation ${ }^{25,26}$. Involvement of these two pathways in the COX-2 mediated hypertrophic differentiation was determined by immunoblotting (Figure 5.4E). In keeping with involvement in chondrocyte hypertrophy, the level of active phospho-Smad1/5/8 increased in the BMP-2 condition (Figure 5.4E; lanes 1 vs. 3). However, inhibition of COX-2 activity by NS398 did not influence the phosphorylation level of Smad1/5/8 (Figure 5.4E; lanes 1 vs. 2 and 3 vs. 4). Canonical Wnt-signalling transcriptionally regulates Runx2 levels via b-catenin. Whereas BMP-2 only marginally enhanced b-catenin levels (Figure 5.4E, short exposure; lanes 1 vs. 3), inhibition of COX-2 activity caused a sharp decline in b-catenin levels in both the absence as well as the presence of BMP-2 (Figure 5.4E; lane 1 vs. 2 and 3 vs. 4), consistent with a possible regulatory role for COX-2 activity in chondrocyte hypertrophy via Wnt/b-catenin-signaling.

In the above presented data COX-2 was inhibited from onset of differentiation $(\mathrm{t}=0)$ onward. However, COX-2 protein expression during ATDC5 differentiation is first detectable at day 7 onward (Figure 5.1B). To determine a possible window of action, we therefore asked whether inhibition of COX-2 at later differentiation stages would show the inhibitory effect on chondrocyte hypertrophy. Differentiation medium was supplemented with $20 \mathrm{mM}$ NS398 from day 7 onward and chondrogenic differentiation was monitored 
until day 21. Analyses of hypertrophic marker expression (Col10a1, Runx2, Mef2c, Mmp13, VEGF-A and Alp) revealed that inhibition of COX-2 caused significantly lower upregulation of all markers at 14 and or 21 days in differentiation (Figure 5.5). Of note, NS398 did not significantly influence Col2al mRNA expression at day 14, whereas it caused a significantly higher upregulation further in differentiation (day 21). Combined, these results clearly show that inhibition of COX-2 activity specifically affects late, hypertrophic chondrocyte differentiation, independently of early chondrogenic commitment.
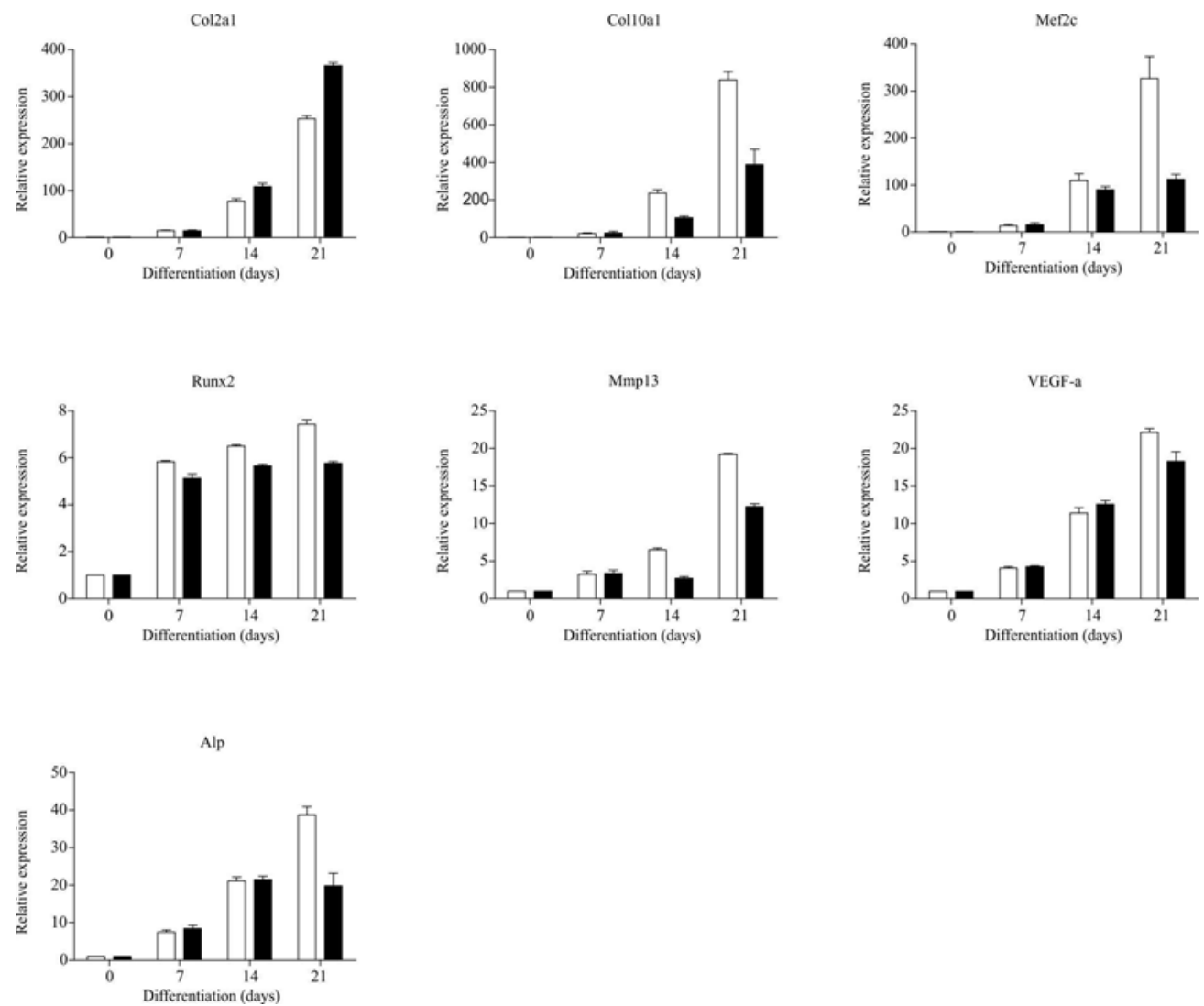

Figure 5.5: Inhibition of $\mathrm{COX}-2$ activity decreases chondrocyte hypertrophic differentiation independent of the differentiation phase

ATDC5 cultures were differentiated for 7 days. After 7 days COX-2 activity was continuously inhibited by addition of NS398 until day 21 into differentiation. In day 0, 7, 14 and 21 samples, gene expression of Col2a1, Col10a1, Runx2, Mef2c, Mmp13, VEGF-a and Alp was determined. For every condition and time point 8 individual samples were measured. White bars indicate the control (DMSO) and black bars represent the $20 \mathrm{mM}$ NS398 condition. $*$ indicates $\mathrm{p}<0.05$. 


\subsection{Inhibition of $\mathrm{COX}-2$ activity does not affect chondrocyte cell proliferation}

As proliferation is a crucial aspect of ATDC5 chondrogenic differentiation ${ }^{23}$, we addressed a potential effect of COX-2 inhibition on cell proliferation under proliferation and chondrogenic conditions. To this end we generated growth curves with increasing NS398 concentrations $(0,0.2,2$ and $20 \mathrm{mM})$. Under proliferation conditions, cell numbers increased approximately 15 fold over a 14 day time span (Figure 5.6A). None of the NS398 concentrations affected cell proliferation. Under chondrogenic conditions, cellular proliferation rate is significantly increased over normal proliferation conditions (Figure 5.6B). Again, NS398 did not affect cell proliferation rate at any of the tested concentrations under differentiation conditions. Overall these data show that specific inhibition of COX-2 activity during ATDC5 proliferation and chondrogenic differentiation does not affect the proliferative capacity of ATDC5 and thus excludes an effect of NS398 on hypertrophic differentiation through impaired proliferation capacity.

A

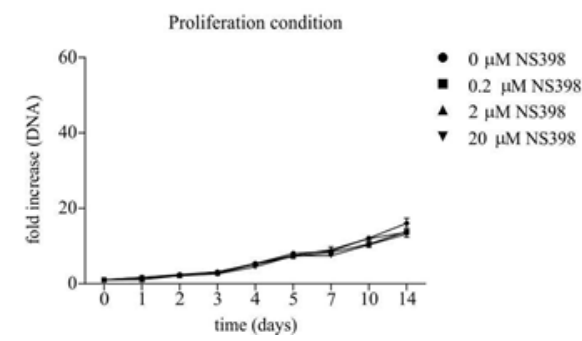

B

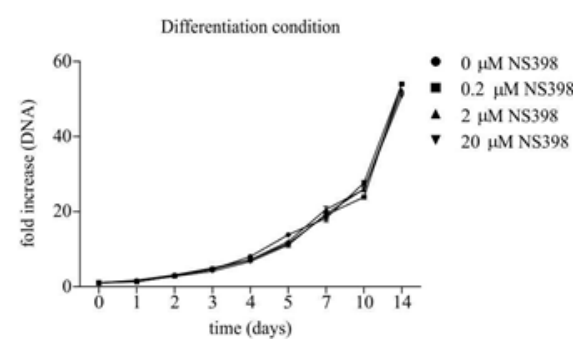

Figure 5.6: Influence of COX-2 inhibition on ATDC5 proliferation rate

Proliferation speed of ATDC5 cells was determined under either proliferation (A) or differentiation (B) conditions and in the presence of increasing concentrations of NS398. Crystal-violet staining was conducted collectively on fixed day $0,1,2,3,4,5,7,10$ and 14 samples and absorbance of the eluates was determined spectrophotometrically. Fold increase (DNA) from samples was calculated relatively to day 0 . The data points represent the average of 6 independent samples and error bars represent mean \pm s.d..

\subsection{Inhibition of chondrocyte hypertrophic differentiation in PAC}

To verify the anti-hypertrophic effect of COX-2 inhibition in vitro, we adopted an ex vivo periosteal organ culture system suitable to display chondrogenic potential ${ }^{24}$. Importantly, the use of periosteal tissue is directly relevant for endochondral ossification processes ${ }^{27}$. The mesenchymal progenitor cell-containing tissue is cultured in between agarose layers 
(periosteum agarose culture: PAC), and induced to differentiate by transforming growth factor $\beta 3$ (TGF- $\beta 3$ ). Chondrogenic differentiation of PACs was evident after 20 days, as visualized by safranin-O staining of tissue sections. Increasing intensity of safranin-O staining after 40 culture days indicates increased proteoglycan deposition (Figure 5.7A). TGF-b is absolutely required for the PAC model, as in its absence mature chondrocytes are not formed and cartilage matrix deposition is not detectable at 20 days $\left({ }^{24}\right.$ and Figure 5.7B; left panel). In agreement with a prominent role for BMP-2 in chondrocyte hypertrophy, combined treatment with TGF- $\beta 3$ and BMP-2 clearly resulted in a hypertrophic PAC morphology at 20 days (Figure 5.7B; right panel) as compared to single treatment with TGF- $\beta 3$ (Figure 5.7B; middle panel) or BMP-2 alone (data not shown). Gene expression analyses confirmed that chondrocyte hypertrophy in the PAC system completely dependents on BMP-2: Col10a1 mRNA (Figure 5.7C; middle panel) and Runx2 protein (Figure 5.7D) are only induced when TGF- $\beta 3$ is combined with BMP-2. Consistent with its requirement for chondrogenesis, TGF- $\beta 3$-only treated PACs show moderate Col2a1 mRNA induction, which is more than 3 -fold enhanced by combination treatment with BMP-2 (Figure 5.7C; upper panel).

To analyze the relationship between COX-2 and chondrocyte hypertrophy in PACs, COX-2 gene expression was determined: TGF- $\beta 3$ induced COX-2 expression, which is increased almost 2-fold by BMP-2 (Figure 5.7C; lower panel), confirming that chondrocyte hypertrophic differentiation also correlates to $\mathrm{COX}-2$ levels in an independent ex vivo model. To further substantiate the requirement of COX-2 activity for hypertrophic differentiation, the effect of NS398 on the PAC model (with BMP-2 treatment) was evaluated. Two micromolar NS398 did not alter proteoglycan deposition (Figure 5.7E, upper right panel) and correspondingly Col2a1 expression was not affected (Figure 5.7E, Col2a1 panel sets and Figure 5.7F, upper panel), consistent with our in vitro findings. In contrast, NS398 treatment strongly reduced Col10al expression (Figure 5.7E, Col10a1 panel sets and Figure 7F, lower panel), Alp expression (Figure 5.7E, Alp panel sets) and prevented the hypertrophy-associated chondrocyte morphological changes in PACs (Figure 5.7E, upper right panel). COX-2 inhibition was confirmed by an almost complete lack of $\mathrm{PGE}_{2}$ synthesis in the NS398 condition (Figure 5.7G). 
A

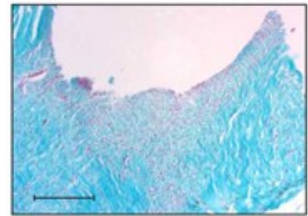

0 days (fresh periosteum)

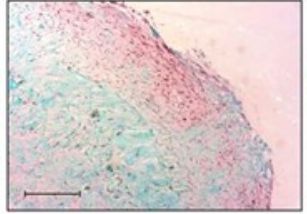

10 days

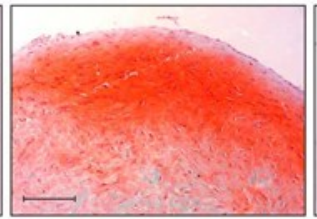

20 days

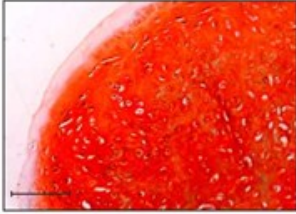

40 days

B

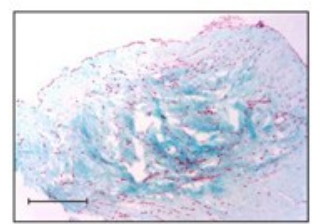

(-) TGFß3 (-) BMP-2

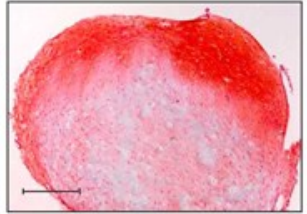

(+) TGFß3 (-) BMP-2

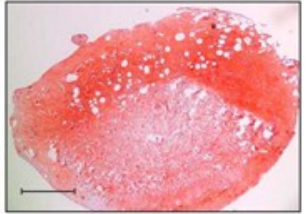

(+) TGFß3 (+) BMP-2

C

Col2al

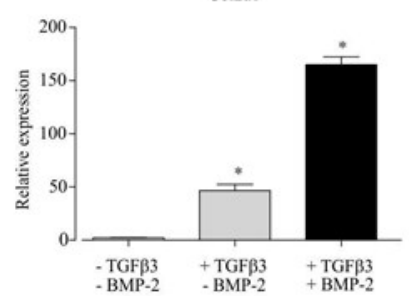

D

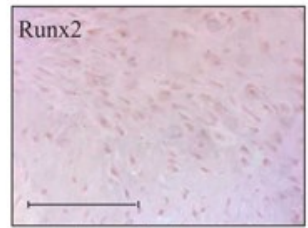

$(+)$ TGF $\beta 3(-)$ BMP-2
Colloal

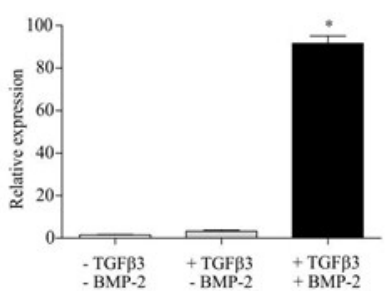

Cox-2

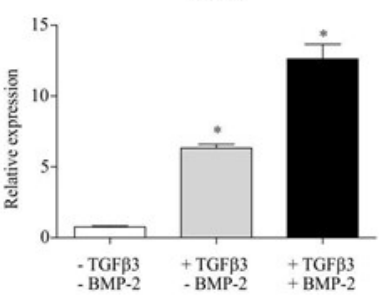

(+) TGFß3 (+) BMP-2

Figure 5.7: BMP-2 mediated chondrocyte hypertrophy in PAC is decreased by COX-2 inhibition

(A). Periosteum agarose cultures (PAC's) were differentiated for 0, 10, 20 or 40 days as previously described previously ${ }^{24}$. Five micrometer sections were stained with Safranin-O/Fast green (bars represent $\left.100 \mathrm{~mm}\right) .(B)$. PAC's were differentiated in the absence of TGF-b3, in the presence of TGF-b3 $(10 \mathrm{ng} / \mathrm{ml})$ or in the presence of TGF-b3 (10 ng/ml) and BMP-2 (30 ng/ml). The panel shows representative examples of PAC tissue sections (20 days) stained with Safranin-O/Fast (bars represent $1 \mathrm{~mm}$ ). $(C)$. Conditions mentioned in $(\mathrm{B})$. were also analyzed quantitatively by RT-qPCR for induction of Col2a1, Col10a1 and COX2 mRNA expression. Bars represent the average of 6 individual PAC samples and error bars represent mean \pm s.d.. (D). Hypertrophic differentiation of these samples was assessed by immunohistochemical staining of Runx2 (bars represent $100 \mathrm{~mm}$ ). (E). PACs were differentiated (20 days) in the presence of TGF-b3 and BMP-2 with or without supplementation of NS398 (2 
E
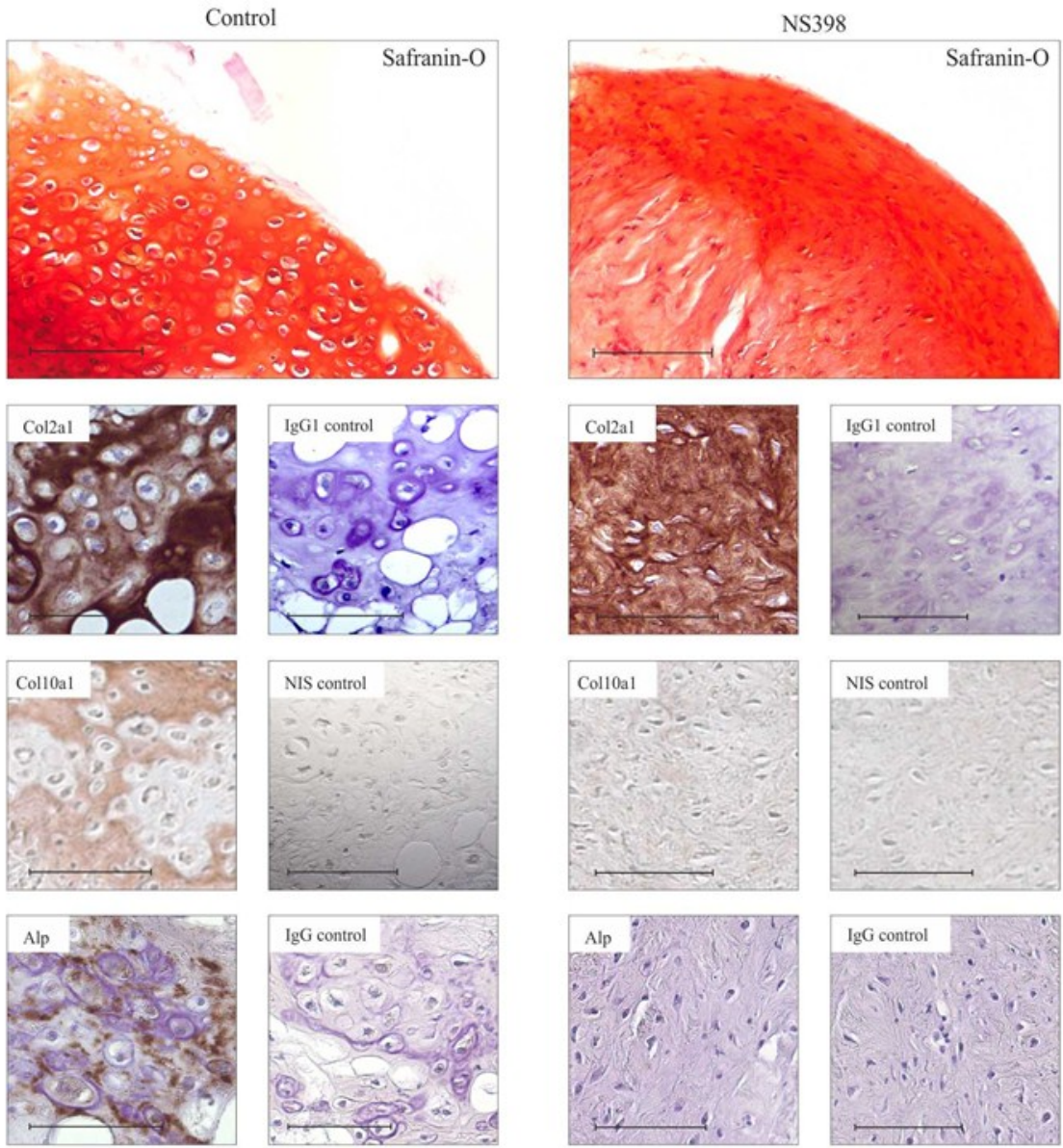

$\mathrm{F}$
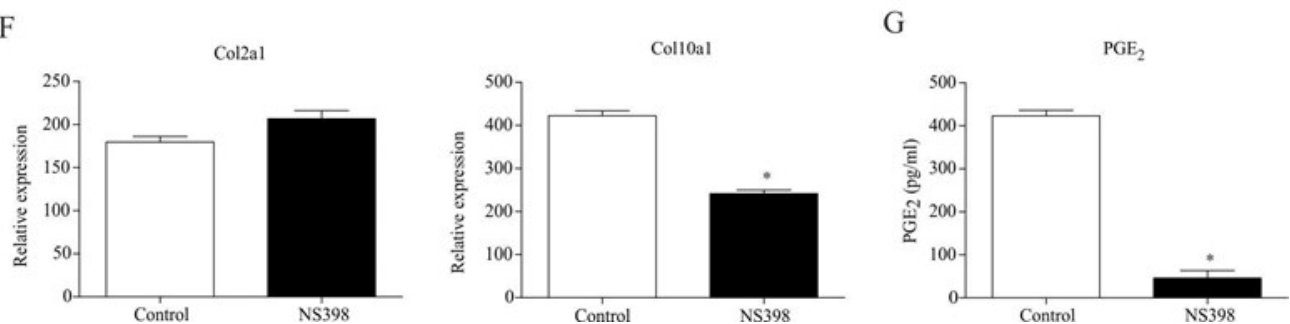

$\mathrm{mM}$ ). Tissue sections ( $5 \mathrm{~mm}$ ) were stained with Safranin-O/Fast green (top micrographs). Immunohistochemical stainings were performed to visualize Col2al (upper micrographs), Col10a1 (middle micrographs) and Alp (lower micrographs) (bars represent $100 \mathrm{~mm}$ ). Negative controls are depicted for each immuno staining: negative IgG1 control for Col2a1; non-immune rabbit serum (NIS) for Col10a1 and non-immune goat IgG's for Alp. $(F)$. PACs were differentiated in conditions as used in (E). and induction of Col2a1 and Col10a1 expression was determined. Bars represent the average of 6 individual PAC samples and error bars indicate mean \pm s.d.. $(G)$. Inhibition of COX-2 activity was confirmed in samples from (F). by measuring PGE $_{2}$ synthesis (pg/ml). Absolute values are shown. $*$ indicates $\mathrm{p}<0.05$. 


\subsection{Selective inhibition of COX-2 impairs chondrocyte hypertrophic differentiation in the} growth plate

To further corroborate our findings in a developmentally and pharmacologically relevant in vivo context, we determined whether treatment with a clinically relevant selective COX-2 inhibitor, celecoxib, affected growth plate development in vivo. Celecoxib selectively inhibited COX-2 over COX-1 (Figure 5.8A). A dose-response analysis confirmed a similar anti-hypertrophic action for celecoxib as for NS398: $10 \mathrm{mM}$ celecoxib significantly decreased Col10a1 and ALP mRNA upregulation at day 14 in ATDC5 differentiation, whereas it showed no negative effect on Col2a1 mRNA expression (Figure 5.8B).

A Celecoxib selectivity

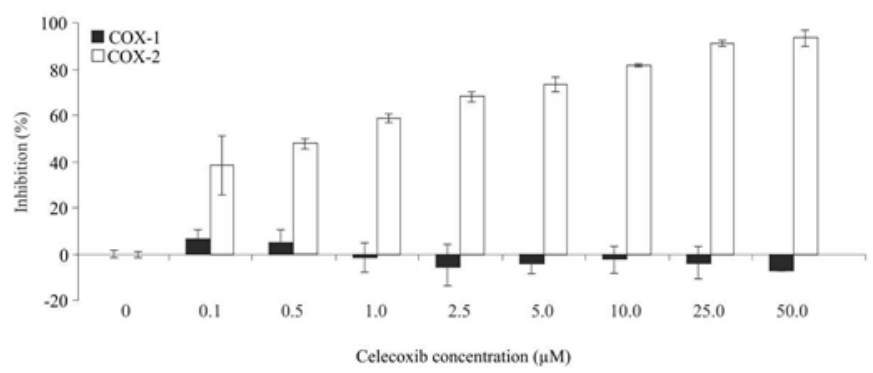

B
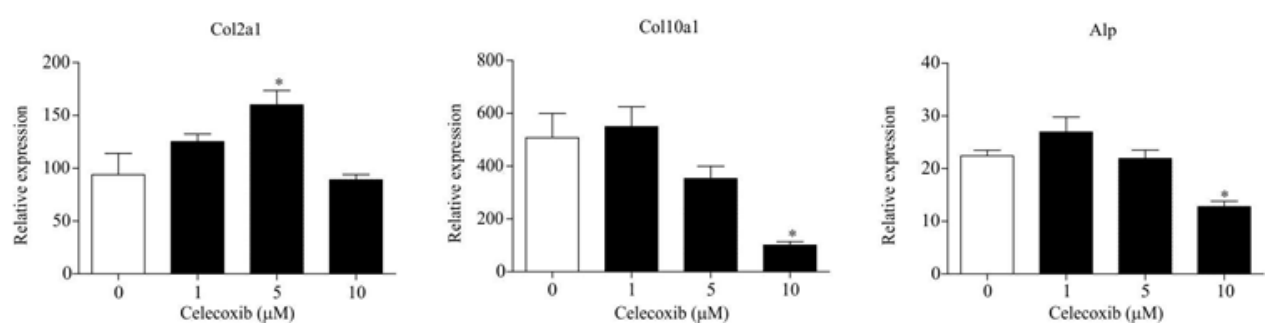

Figure 5.8: Celecoxib properties

(A). The selectivity of Celecoxib for COX-2 over COX-1 was determined using a COX-1/COX-2 colorimetric inhibition assay. Celecoxib was tested at $0,0.1,0.5,1.0,2.5,5.0,10.0,25.0$ and $50.0 \mu \mathrm{M}$. The inhibitory capacity was determined as "percent inhibition" compared to a maximum activity control condition. Bars represent average inhibition percentages of COX-1 (black bars) and COX-2 (white bars). Measurements were done in triplicate and error bars represent the variances in inhibitory activity. (B). Increasing concentrations of celecoxib were added to differentiating ATDC5 cultures. Messenger RNA expression of Col2a1, Col10a1 and Alp in day 14 samples was determined. Bars represent mean \pm s.d.. * indicates $\mathrm{p}<0.05$. 
To determine the consequences of selective COX-2 inhibition on growth plate development, skeletally immature New Zealand white rabbits were systemically treated with celecoxib for 3 weeks (six animals in control group and seven animals in celecoxib group). In blood plasma we confirmed that celecoxib efficiently inhibited in vivo $\mathrm{PGE}_{2}$ synthesis and thus systemically inhibited COX-2 (Figure 5.9A). Growth plate sections of the ulnae were stained with Haematoxilin (Figure 5.9B). Analysis of the total growth plate thickness of control sections revealed an average thickness of $451.8 \mathrm{~mm}$ (Figure 5.9C). In contrast, the average thickness of the growth plates of the celecoxib-treated group was significantly reduced to $421.3 \mathrm{~mm}$ (Figure 5.9B/C). To discern a potential effect of celecoxib on early versus late chondrogenic differentiation stages, the thickness of the proliferative and hypertrophic zones were measured separately. Importantly, and consistent with our in vitro and ex vivo findings, the length of the proliferative zones of both the control and celecoxib-treated group did not differ significantly (Figure 5.9B/C). In contrast, the hypertrophic zone of the celecoxib-treated group was significantly thinner as compared to the control group (113.8 mm vs $148.4 \mathrm{~mm}$, respectively; Figure 5.9B/C). The ratio's of hypertrophic/proliferative zone length of control and celecoxib treated growth plates were 0.495 and 0.370 , respectively (Figure 5.9D). These ratio's differ significantly from each other and confirms reduced hypertrophic zone length. The difference in total growth plate thickness thus resulted from impaired development of the hypertrophic zone. Taken together, these in vivo data clearly show that inhibition of COX-2 activity during skeletal development affects growth plate development at the chondrocyte hypertrophic level and provides further evidence in support of a crucial role of COX-2 activity during chondrocyte hypertrophic differentiation in an endochondral ossification context. 
A

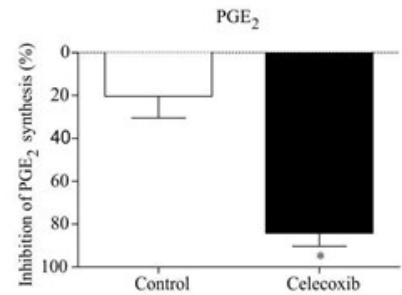

C

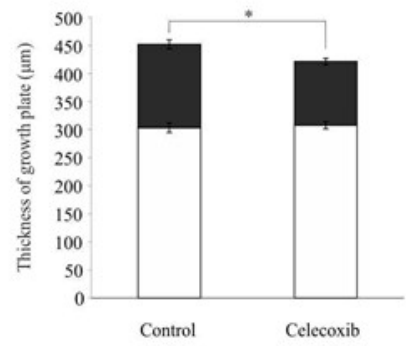

\begin{tabular}{|c|c|c|}
\hline Total thickness & $451.8 \mu \mathrm{m}$ & $421.3 \mu \mathrm{m}$ \\
\hline - Hypertrophic & $148.4 \mu \mathrm{m}$ & $113.8 \mu \mathrm{m}$ \\
\hline$\square$ Proliferative & $303.4 \mu \mathrm{m}$ & $307.5 \mu \mathrm{m}$ \\
\hline
\end{tabular}

B

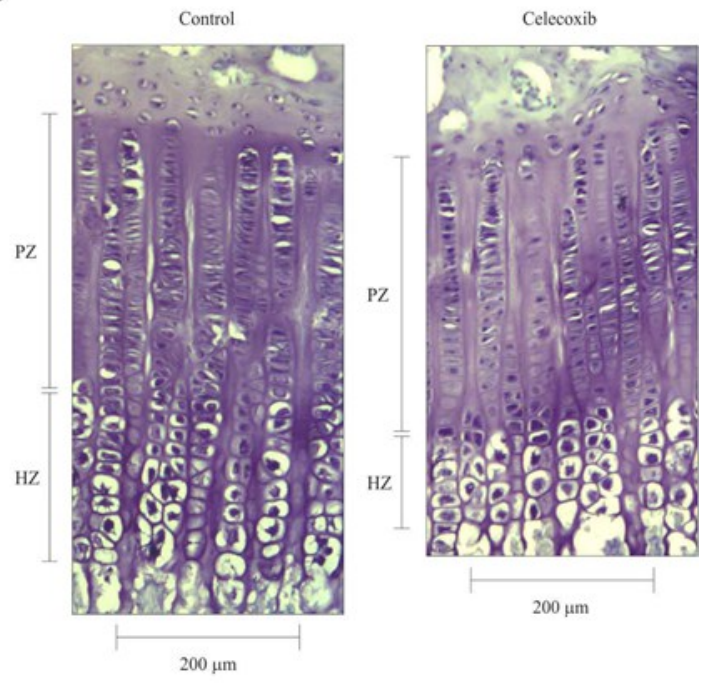

D

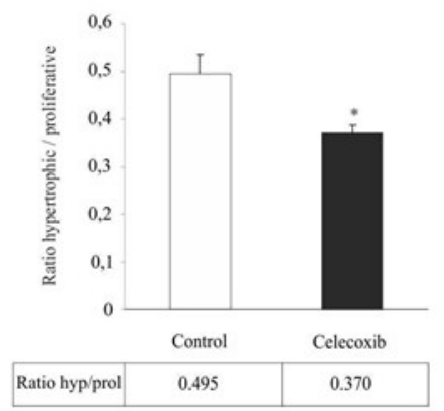

Figure 5.9: Chondrocyte hypertrophic differentiation in the rabbit growth plate is impaired by inhibition of COX -2 activity

Skeletally immature New Zealand white rabbits received Celecoxib (7 animals) on a daily basis. Six control animals were used. Rabbits were treated for three weeks. (A). COX-2 inhibition was confirmed by measuring $\mathrm{PGE}_{2}$ levels in serum at 3 weeks. Inhibition of $\mathrm{PGE}_{2}$ synthesis was determined as \% decrease as compared to pretreatment serum samples. $(B)$. Representative examples of growth plate sections of control and celecoxib treated rabbits. $\mathrm{PZ}=$ proliferative zone, $\mathrm{HZ}=$ hypertrophic zone. $(C)$. Quantification of growth plate zones of both groups. Normal distribution of the data was confirmed by a Shapiro-Wilk test. White bars: proliferative zone, black bars: hypertrophic zones. Average total thickness control group $=451.8 \mu \mathrm{m}$; celecoxib group $=421.3 \mu \mathrm{m}$ (Mann-Whitney, $\mathrm{p}=0.032$ ). PZ control group $=303.4 \mu \mathrm{m}$; PZ celecoxib group $=307.5 \mu \mathrm{m}$ (Mann-Whitney, $\mathrm{p}=0.668)$. HZ control group $=148.4 \mu \mathrm{m} ; \mathrm{HZ}$ celecoxib group $=113.8 \mu \mathrm{m}$ (Mann-Whitney, $\mathrm{p}=0.046)$. $(D)$. Ratio's between the hypertrophic and proliferative zones of the control and celecoxib groups were determined (Mann-Whitney, $\mathrm{p}=0.013$ ). * indicates $\mathrm{p}<0.05$. 


\section{Discussion}

The involvement of COX-2 in osteogenic differentiation is well documented in literature. We here also demonstrate a role for COX-2 in the chondrogenic phase of endochondral ossification. Using three independent models we show that late chondrocyte hypertrophy in endochondral ossification is, at least in part, determined by COX-2 activity. We provide evidence that COX-2 function intersects with BMP-2 and $W n t / \beta$-catenin signalling. Importantly, the finding that the effect of $\mathrm{COX}-2$ inhibition appears restricted to chondrocyte hypertrophic differentiation has important implications for the clinical application of NSAIDs.

\subsection{COX-2 in chondrocyte hypertrophy}

We demonstrate that COX-2 is expressed in the growth plate, specifically in the prehypertrophic and hypertrophic compartments of the mouse growth plate. Despite apparently discrepant COX-2 staining pattern in the rat growth plate ${ }^{18,19}$, both data sets support a role for COX-enzymes in growth plate development. In addition, our in vivo findings demonstrate that systemic COX-2 inhibition during skeletal development results in abnormal development of the hypertrophic zone of the growth plate. This data is consistent with our observations that in in vitro and ex vivo models COX-2 specific NSAIDs decrease chondrocyte hypertrophy. Impaired Wnt/ $\beta$-catenin signalling was shown to cause defective growth plate hypertrophic differentiation ${ }^{28}$ and postnatal cartilage development ${ }^{29}$. These and our findings predict that the COX-2 -/- mouse ${ }^{13}$ should display a, most likely, subtle skeletal developmental phenotype. Although the presence of functionally redundant mechanisms could theoretically explain lack of effects, detailed re-analysis of growth plates in COX-2 -/- mice, may reveal a mild phenotype. Conversely, although we could detect a significant quantitative difference in hypertrophic zone length between celecoxib and non-treated animals, it remains to be determined whether the effects of transient COX-2 inhibition results in persistent skeletal abnormalities into adulthood.

\subsection{COX-2 - integration into known chondrogenic signalling}

Hypertrophic chondrocyte differentiation can be monitored by expression of several important markers like Runx2, Col10a1 and ALP. Direct transcriptional regulation of the Col10a1 gene is regulated by Runx $2^{22}$ and transcriptional activity of Runx 2 on the 
Col10a1 promoter is cooperatively controlled by BMP-2-induced Smad's ${ }^{15}$. In addition, in osteogenic differentiation phosphorylation has been described as an important determinant for transcriptional activity of Run $2^{30}$. Chondrocyte hypertrophic differentiation is, at least in part, regulated via Smad1/5/8 signalling ${ }^{26}$. As we could not detect any difference on Smad1/5/8 phosphorylation between control and NS398-treated samples, this suggests that COX-2 either acts in parallel to or down-stream of R-Smads. Indeed we found experimental evidence that support a down-stream relation. Inhibition of Smad1/5/8 phosphorylation by dorsomorphin inhibited COX-2 expression and subsequent $\mathrm{PGE}_{2}$ synthesis (Figure 5.10). Concurrent with decreased detection of $\mathrm{PGE}_{2}$ and reduced Runx2 and Col10a1 levels, BMP-2 mRNA levels are reduced by NS398 in a dose-dependent manner. Hence, the NS398-mediated decrease of Col10a1 expression is consistent with reduced Runx2 expression. Upstream of Runx2 we found that b-catenin levels negatively respond to inhibition of COX-2 activity. Prostaglandin receptors are G-protein coupled receptors that activate protein kinase A (PKA) and in turn control b-catenin levels via glycogen synthase kinase 3 (GSK-3) activity ${ }^{31,32}$. We did not address phosphorylation of

A

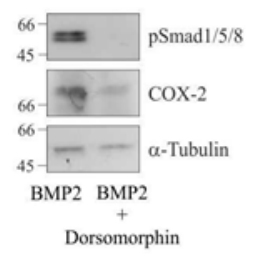

B

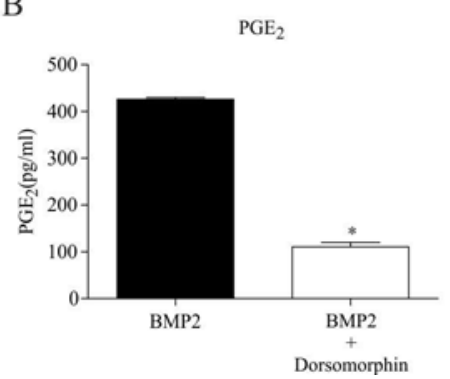

C

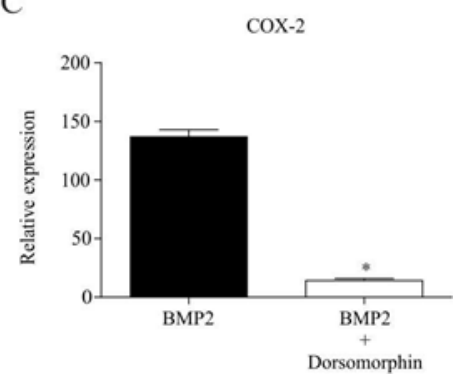

Figure 5.10: Smad1/5/8 activity and COX-2

To determine whether BMP-2 induced Smad1/5/8 activity regulates COX-2 we employed dorsomorphin (10 $\mathrm{mM})$ on ATDC5 cells in the presence of BMP-2 $(30 \mathrm{ng} / \mathrm{ml})$. (A). Differentiating ATDC5 cells were treated with BMP-2 and BMP-2 + Dorsomorphin for one day. Protein extracts were analyzed by immunoblotting for phosphorylation (= activation) of Smad1/5/8 and expression of COX-2. Lack of detection of p-Smad1/5/8 confirmed dorsomorphin functionality. Decreased expression of COX-2 accompanied inhibition of Smad1/5/8 phosphorylation. $(B)$. To confirm decreased total COX-2 activity, $\mathrm{PGE}_{2}$ levels were determined in culture supernatants. Decreased $\mathrm{PGE}_{2}$ levels confirm the dorsomorphin-induced COX-2 decrease. $(C)$. To place the above finding in a chondrocyte hypertrophic context, ATDC5 cells were differentiated for 14 days in the presence of BMP-2 and Dorsomorphin was added from day 7 on (comparable to ${ }^{26}$ ). Confirming above data, Inhibition of Smad1/5/8 phosphorylation resulted in a significantly lower expression of COX-2 mRNA as compared to the BMP-2 control. 
Runx2 in this study. However, taking the previously reported involvement of canonical Wnt-signalling in the transcriptional regulation of Runx2 expression upon BMP-2 ligand binding into account ${ }^{25}$, this provides, at least in part, a mechanistic explanation for how inhibition of COX-2 activity reduces b-catenin levels and leads to reduced chondrocyte hypertrophic maturation.

Combined with data from other groups our findings $\mathrm{s}^{10,15,33,34}$ strongly support a functional relation between $\mathrm{COX}-2, \mathrm{PGE}_{2}$ and BMP-2 in chondrocyte hypertrophy. Chondrocyte hypertrophic differentiation is induced by BMP-2 during chondrogenesis ${ }^{33}$. BMP-2 induces COX-2 expression and the increased hypertrophic differentiation by exogenous BMP-2 supplementation was diminished by inhibition of COX-2 activity. In addition, endogenous BMP-2 mRNA levels negatively responded to COX-2 inhibition ( ${ }^{10}$ and this study), while endogenous BMP-2 mRNA levels were even further decreased in the presence of exogenous BMP-2. Although it remains currently unclear why BMP-2 treatment increases the NS398 responsiveness of hypertrophic markers, it is possible that a negative feedback effect of exogenous BMP-2 on endogenous BMP-2 levels renders hypertrophic differentiation more susceptible to COX-2 inhibition. In contrast to the here reported anti-hypertrophic effect of COX-2 inhibition, $\mathrm{PGE}_{2}$ has been demonstrated to delay (BMP-2 induced) hypertrophic differentiation via PKA and protein kinase C (PKC) signalling ${ }^{35,36}$. These conflicting results may be related to the choice of experimental models: whereas we used chondroprogenitors, PKA/PKC involvement was tested in mature chondrocyte cultures. In relation to this, differentially regulated COX-2 expression during different stages of chondrogenesis was previously suggested ${ }^{37,38}$. Importantly, as COX-2 inhibition leads to an indiscriminate shutdown of prostaglandin synthesis, we cannot rule out the possibility that different prostaglandins contribute differently to chondrocyte differentiation.

\subsection{Clinical use of NSAIDs}

Clinically, NSAIDs are used to block the prostaglandin synthesizing enzymes COX-1 and/ or COX-2. However, the therapeutic application of NSAIDs in the context of bone fracture healing is currently under debate as COX-inhibition by NSAIDs is reported to delay fracture healing or cause non-union in patients and animal models ${ }^{4-8}$. COX-2 is reported to be expressed in fracture callus ${ }^{13,39}$ and molecular genetic mouse models showed that COX-2 and mPGES-1 activity are critically required for bone fracture union ${ }^{13,14}$. Although there is increasing evidence in support of a role for COX-2 in the chondrogenic phase of 
endochondral ossification, most studies focused on COX-2 function in the context of the osteogenic phase of endochondral ossification (i.e. osteoblast and osteoclast biology). Here we identified chondrocyte hypertrophy as another important target of COX-2 activity during the proces of endochondral ossification. Recent work by Petit and colleagues suggests that expression of COL10A1 mRNA in human bone marrow mesenchymal stem cells is decreased by specific inhibition of COX-1 and 5-LOX (5-lypoxygenase) $)^{40}$, suggesting similar mechanisms as presented in our present COX-2 study. However, experimental cell models differ between studies and whether or how COX-1 and COX-2 work together or fulfil separate roles in hypertrophic differentiation remains to be determined.

In conclusion, we here show that the hypertrophic action of BMP-2 on endochondral differentiating chondrocytes is, at least in part, determined via COX-2 activity. This finding may have multiple clinical implications. Firstly, the negative effects of COX-2 inhibition on growth plate development in skeletally immature animals warrants critical reevaluation of the clinical context in which NSAIDs and pharmacological equivalents are applied. Secondly, impaired bone fracture healing by NSAIDs may also be explained by impaired chondrogenic differentiation, in addition to the currently known impaired osteogenic differentiation alone.

\section{Acknouledgements}

The authors appreciate the statistical assistance of Dr. I. Punt (Dept. Orthopaedic Surgery, Maastricht University Medical Center, the Netherlands). Dr. M. Rutgers (Dept. Orthopaedic Surgery, University Medical Center Utrecht, the Netherlands) and Dr. M. Tryfonidou (Dept. of Clinical Sciences of Companion Animals, Utrecht University, the Netherlands) for help with immunohistochemistry. The authors also thank Dr. G. Lunstrum (Shriners Hospital for Children, Portland, USA) for providing the anti-Collagen type X serum for immunohistochemistry. This work is financially supported by the Dutch Arthritis Association (grant LLP14) and the Dutch Stichting Annafonds|NOREF (grants 07/07 and 08/42). Recombinant BMP-2 was a generous gift from Wyeth Pharmaceuticals. 
6. References

1 Kronenberg, H. M. Developmental regulation of the growth plate. Nature 423, 332-336 (2003).

2 Mackie, E. J., Ahmed, Y. A., Tatarczuch, L., Chen, K. S. \& Mirams, M. Endochondral ossification: how cartilage is converted into bone in the developing skeleton. Int J Biochem Cell Biol 40, 46-62 (2008).

3 Brooks, P. et al. Interpreting the clinical significance of the differential inhibition of cyclooxygenase-1 and cyclooxygenase-2. Rheumatology (Oxford) 38, 779-788 (1999).

4 Thaller, J., Walker, M., Kline, A. J. \& Anderson, D. G. The effect of nonsteroidal anti-inflammatory agents on spinal fusion. Orthopedics 28, 299-303; quiz 304295 (2005).

5 Gerstenfeld, L. C. et al. Differential inhibition of fracture healing by non-selective and cyclooxygenase-2 selective non-steroidal anti-inflammatory drugs. J Orthop Res 21, 670-675 (2003).

6 Herbenick, M. A., Sprott, D., Stills, H. \& Lawless, M. Effects of a cyclooxygenase 2 inhibitor on fracture healing in a rat model. Am J Orthop (Belle Mead NJ) 37, E133-137 (2008).

7 Goodman, S. et al. COX-2 selective NSAID decreases bone ingrowth in vivo. $J$ Orthop Res 20, 1164-1169 (2002).

8 Simon, A. M. \& O'Connor, J. P. Dose and time-dependent effects of cyclooxygenase-2 inhibition on fracture-healing. J Bone Joint Surg Am 89, 500-511 (2007).

9 Grohs, J. G., Schmidt, M. \& Wanivenhaus, A. Selective COX-2 inhibitor versus indomethacin for the prevention of heterotopic ossification after hip replacement: a double-blind randomized trial of 100 patients with 1-year follow-up. Acta Orthop 78, 95-98 (2007).

10 Arikawa, T., Omura, K. \& Morita, I. Regulation of bone morphogenetic protein-2 expression by endogenous prostaglandin E2 in human mesenchymal stem cells. $J$ Cell Physiol 200, 400-406 (2004).

11 Einhorn, T. A. Cox-2: Where are we in 2003? - The role of cyclooxygenase-2 in bone repair. Arthritis Res Ther 5, 5-7 (2003).

$12 \mathrm{Xie}, \mathrm{C}$. et al. Rescue of impaired fracture healing in COX-2-/- mice via activation of prostaglandin E2 receptor subtype 4. Am $J$ Pathol 175, 772-785, doi:ajpath.2009.081099 [pii]

10.2353/ajpath.2009.081099 (2009).

13 Zhang, X. et al. Cyclooxygenase-2 regulates mesenchymal cell differentiation into the osteoblast lineage and is critically involved in bone repair. $J$ Clin Invest 109, 1405-1415 (2002).

14 Yamakawa, K. et al. Association of microsomal prostaglandin E synthase 1 deficiency with impaired fracture healing, but not with bone loss or osteoarthritis, in mouse models of skeletal disorders. Arthritis Rheum 58, 172-183, doi:10.1002/ art.23158 (2008).

15 Javed, A. et al. Specific residues of RUNX2 are obligatory for formation of BMP2-induced RUNX2-SMAD complex to promote osteoblast differentiation. Cells Tissues Organs 189, 133-137 (2009).

16 Knippenberg, M., Helder, M. N., de Blieck-Hogervorst, J. M., Wuisman, P. I. \& Klein-Nulend, J. Prostaglandins differentially affect osteogenic differentiation of 
human adipose tissue-derived mesenchymal stem cells. Tissue Eng 13, 2495-2503 (2007).

17 Jakob, M., Demarteau, O., Suetterlin, R., Heberer, M. \& Martin, I. Chondrogenesis of expanded adult human articular chondrocytes is enhanced by specific prostaglandins. Rheumatology (Oxford) 43, 852-857 (2004).

18 Brochhausen, C., Neuland, P., Kirkpatrick, C. J., Nusing, R. M. \& Klaus, G. Cyclooxygenases and prostaglandin E2 receptors in growth plate chondrocytes in vitro and in situ--prostaglandin E2 dependent proliferation of growth plate chondrocytes. Arthritis Res Ther 8, R78 (2006).

19 Brochhausen, C. et al. Cyclooxygenases (COX-1 and COX-2) for tissue engineering of articular cartilage--from a developmental model to first results of tissue and scaffold expression. Biomed Mater Eng 18, 15-23 (2008).

20 De Luca, F. et al. Regulation of growth plate chondrogenesis by bone morphogenetic protein-2. Endocrinology 142, 430-436 (2001).

21 Linsenmayer, T. F., Eavey, R. D. \& Schmid, T. M. Type X collagen: a hypertrophic cartilage-specific molecule. Pathol Immunopathol Res 7, 14-19 (1988).

22 Zheng, Q. et al. Type X collagen gene regulation by Runx2 contributes directly to its hypertrophic chondrocyte-specific expression in vivo. J Cell Biol 162, 833842, doi: $10.1083 /$ jcb.200211089 jcb.200211089 [pii] (2003).

23 Atsumi, T., Miwa, Y., Kimata, K. \& Ikawa, Y. A chondrogenic cell line derived from a differentiating culture of AT805 teratocarcinoma cells. Cell Differ Dev 30, 109-116 (1990).

24 O'Driscoll, S. W., Recklies, A. D. \& Poole, A. R. Chondrogenesis in periosteal explants. An organ culture model for in vitro study. J Bone Joint Surg Am 76, 1042-1051 (1994).

25 Dong, Y. F., Soung do, Y., Schwarz, E. M., O'Keefe, R. J. \& Drissi, H. Wnt induction of chondrocyte hypertrophy through the Runx2 transcription factor. $J$ Cell Physiol 208, 77-86, doi:10.1002/jcp.20656 (2006).

26 Hellingman, C. A. et al. Smad Signaling Determines Chondrogenic Differentiation of Bone-Marrow-Derived Mesenchymal Stem Cells: Inhibition of Smad1/5/8P Prevents Terminal Differentiation and Calcification. Tissue Eng Part $A$, doi:10.1089/ten.TEA.2010.0043 (2011).

27 Dwek, J. R. The periosteum: what is it, where is it, and what mimics it in its absence? Skeletal Radiol 39, 319-323, doi:10.1007/s00256-009-0849-9 (2010).

28 Nagayama, M. et al. Wnt/beta-catenin signaling regulates cranial base development and growth. J Dent Res 87, 244-249, doi:87/3/244 [pii] (2008).

29 Chen, M. et al. Inhibition of beta-catenin signaling causes defects in postnatal cartilage development. $J$ Cell Sci 121, 1455-1465, doi:jcs.020362 [pii] 10.1242/jcs.020362 (2008).

30 Shui, C., Spelsberg, T. C., Riggs, B. L. \& Khosla, S. Changes in Runx2/Cbfa1 expression and activity during osteoblastic differentiation of human bone marrow stromal cells. J Bone Miner Res 18, 213-221, doi:10.1359/jbmr.2003.18.2.213 (2003).

31 Fang, X. et al. Phosphorylation and inactivation of glycogen synthase kinase 3 by protein kinase A. Proc Natl Acad Sci U S A 97, 11960-11965, doi:10.1073/ pnas. 220413597

220413597 [pii] (2000).

32 Hata, A. N. \& Breyer, R. M. Pharmacology and signaling of prostaglandin receptors: multiple roles in inflammation and immune modulation. Pharmacol Ther 103, 147-166, doi:10.1016/j.pharmthera.2004.06.003 
S0163-7258(04)00096-8 [pii] (2004).

33 Steinert, A. F. et al. Hypertrophy is induced during the in vitro chondrogenic differentiation of human mesenchymal stem cells by bone morphogenetic protein-2 and bone morphogenetic protein-4 gene transfer. Arthritis Res Ther 11, R148 (2009).

34 Chikazu, D. et al. Bone morphogenetic protein 2 induces cyclo-oxygenase 2 in osteoblasts via a Cbfal binding site: role in effects of bone morphogenetic protein 2 in vitro and in vivo. J Bone Miner Res 17, 1430-1440 (2002).

35 Clark, C. A. et al. Prostaglandin E2 inhibits BMP signaling and delays chondrocyte maturation. J Orthop Res 27, 785-792 (2009).

36 Li, T. F. et al. PGE2 inhibits chondrocyte differentiation through PKA and PKC signaling. Exp Cell Res 300, 159-169 (2004).

37 Ulivi, V., Giannoni, P., Gentili, C., Cancedda, R. \& Descalzi, F. p38/NF-kBdependent expression of COX-2 during differentiation and inflammatory response of chondrocytes. Journal of cellular biochemistry 104, 1393-1406 (2008).

38 Huh, Y. H., Kim, S. H., Kim, S. J. \& Chun, J. S. Differentiation status-dependent regulation of cyclooxygenase-2 expression and prostaglandin E2 production by epidermal growth factor via mitogen-activated protein kinase in articular chondrocytes. J Biol Chem 278, 9691-9697 (2003).

39 Naik, A. A. et al. Reduced COX-2 expression in aged mice is associated with impaired fracture healing. J Bone Miner Res 24, 251-264 (2009).

40 Petit, A. et al. Novel insights into the mechanism of decreased expression of type $\mathrm{X}$ collagen in human mesenchymal stem cells from patients with osteoarthritis cultured on nitrogen-rich plasma polymers: implication of cyclooxygenase-1. $J$ Biomed Mater Res A 94, 744-750, doi:10.1002/jbm.a.32739 (2010). 



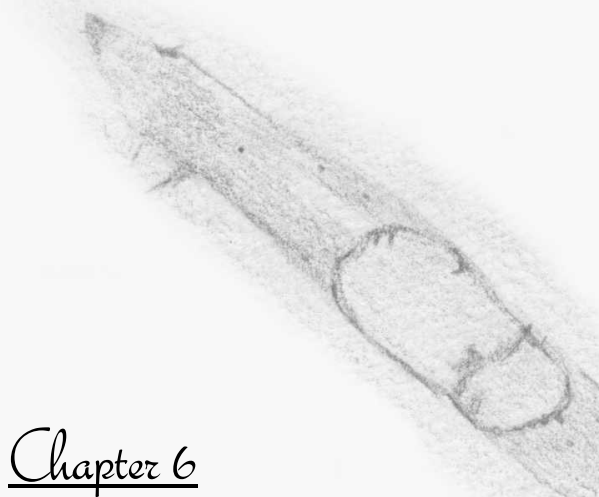

Orostaglandins and Cyclooxygenase-enrymes in Chondrogenic

Differentiation of Progenitor Cells $_{\text {.r. }}$

Prostaglandins and COX-Enzymes in Chondrogenic Differentiation of Progenitor Cells

Marjolein M.J. Caron, Pieter J.Emans, Kathleen Sanen, Don A.M. Surtel, Andy Cremers, Daan Ophelders, Lodewijk W. van Rhijn and Tim J. M. Welting -

Department of Orthopaedic Surgery, Maastricht University Medical Center, Maastricht, the Netherlands (In preparation) 


\section{Abstract}

Introduction: NSAIDs are clinically used to relieve pain and decrease inflammation by inhibition of cyclooxygenase (COX)-catalyzed prostaglandin (PG) synthesis. As PGs are fatty acid mediators involved in bone and cartilage homeostasis, they thereby may provide a novel opportunity to influence cartilage regeneration. We hypothesized that COX-1 and COX-2 have differential roles in the chondrogenic differentiation process of progenitor cells and that their specific inhibition will thereby differently influence the outcome of the differentiation process.

Methodology: ATDC5 cells were differentiated in the presence of different COX-1 (SC-560, Mofezolac) or COX-2 (NS398, Celecoxib) specific inhibitors. COX-1 or COX-2 specificity of the NSAIDs and NSAID-specific inhibition of prostaglandin levels were determined by colorimetric assays and specific ELISAs. Prostaglandins were added during the differentiation process. Gene- and protein expression analyses were employed to determine chondrogenic outcome.

Results: COX-specificity of the NSAIDs was confirmed and for subsequent experiments NSAID concentrations were selected that resulted in similar degrees of COX-1 or COX-2 inhibition. COX-1 specific NSAIDs inhibited Col2a1 and Col10a1 expression. Inhibition of COX-2 resulted in substantial decrease of Col10a1 expression, while Col2a1 remained unaffected. To explain this difference we determined the expression patterns of both COX enzymes as well as specific prostaglandin concentrations during differentiation. COX-1 is upregulated during late chondrogenic differentiation, whereas COX-2 is briefly expressed early in differentiation and increases again late in differentiation. $\mathrm{PGD}_{2}$ and $\mathrm{PGE}_{2}$ followed the COX-2 expression pattern, whereas $\mathrm{PGF}_{2 \alpha}$ and $\mathrm{TXA}_{2}$ levels remained stably low. Furthermore, COX-2 inhibition resulted in decreased levels of all tested PGs, whereas COX-1 inhibition decreased the concentration of all PGs, exept for $\mathrm{PGD}_{2}$ and $\mathrm{PGF}_{2 \alpha}$, which were both increased. Addition of $\mathrm{PGE}_{2}$ and $\mathrm{PGF}_{2 \alpha}$ resulted in increased expression of chondrogenic markers, whereas $\mathrm{TXA}_{2}$ specifically resulted in increased expression of hypertrophic markers.

Conclusions: Our findings point towards a differential role for COX-enzymes and PGproduction in chondrogenic differentiation. Ongoing research is focussing on further elucidating the functional partition of cyclooxygenases and specific prostaglandin production. 


\section{Introduction}

Progenitor cell-based cartilage regeneration is an evolving field and methodologies promoting chondrogenic differentiation of mesenchymal progenitor cells towards chondrocytes and better understanding of the chondrogenic differentiation process is imperative to improve the outcome of cartilage regenerative medicine approaches.

We have previously shown that COX-2 is transiently expressed during the first hours in chondrogenic differentiation of progenitor cells ${ }^{1}$ and again at later time points, when its expression coincidences with chondrocyte extracellular matrix synthesis ${ }^{2}$. In addition, we found that inhibition of COX-2 enzymatic activity results in a reduction of the hypertrophy outcome of the differentiation process ${ }^{2}$. However, whether this observation is specific for COX-2 and how this relates to COX-1 activity is largely unknown.

Cyclooxygenases, also known as prostaglandin $\mathrm{H}$ synthases, are enzymes that catalyse the rate-limiting step in the generation of prostaglandins ${ }^{3}$. Specifically, arachidonic acid is released from membrane phospholipids by phospholipase $\mathrm{A}_{2}$ and serves as a substrate for the cyclooxygenases. Arachidonic acid is oxygenated by COX-activity to form prostaglandin $\mathrm{G}_{2}\left(\mathrm{PGG}_{2}\right)$ and subsequently the peroxidase activity of the COX enzyme drives the reduction of $\mathrm{PGG}_{2}$ to hydroperoxy endoperoxide prostaglandin $\mathrm{H}_{2}\left(\mathrm{PGH}_{2}\right)^{4}$. This unstable intermediate is converted by specific synthases to the main prostaglandin subgroups $\mathrm{PGD}_{2}, \mathrm{PGE}_{2}, \mathrm{PGI}_{2}, \mathrm{PGF}_{2 \alpha}$ and $\mathrm{TXA}_{2}$, which can be converted into various other intermediates $^{5,6}$. To date three COX isoforms have been described: COX-1, COX-2 and COX-3. The first two are the most prevalent en best described isoforms ${ }^{7}$. The COX-3 isoform is a splice variant of COX-1; however there is much debate on the function of COX $-3^{8-10}$. Both COX-1 and COX-2 isoforms catalyse the same enzymatic reactions and are structurally related $^{11}$. They have remarkable differences regarding their tissue distribution, expression levels and their ability to response to various stimuli ${ }^{7,12,13}$. COX-1 is constitutively expressed by most mammalian cells and regarded as the "housekeeping" cyclooxygenase. On the other hand, COX-2 expression is low in most tissues but can be rapidly induced upon exposure to various stimuli as inflammation, mechanical stress and injury $^{3,7}$ due to inducible enhancer elements in its promoter ${ }^{11}$. Studies investigating the roles of cyclooxygenases and the effects of prostaglandins on (in vitro) chondrogenic differentiation have mainly focussed on COX-2 (inhibition) and $\mathrm{PGE}_{2}{ }^{14-20}$. However, the contribution of COX-1 and other prostaglandins is largely ignored. While, given their different 
role in cellular prostaglandin synthesis, it is not unlikely that either of the two COX enzymes are differently involved in determining the outcomes of the chondrogenic differentiation process.

We hypothesized that COX-1 and COX-2 have differential roles in the chondrogenic differentiation process of progenitor cells and that their specific inhibition will thereby differently influence the outcome of the differentiation process. In the present study we show that COX-1 and COX-2 have different expression patterns during chondrogenic differentiation of progenitor cells and that specific inhibition of the COX-1 enzyme results in overall inhibition of chondrogenic differentiation, whereas COX-2 inhibition specifically decreased the level of chondrocyte hypertrophy. Prostaglandin concentration were found to be differentially influenced by COX-1 or COX-2 inhibition and addition of specific prostaglandins modulated the chondrogenic outcome unexpectedly different; $\mathrm{PGF}_{2 \alpha}$ and $\mathrm{PGE}_{2}$ increased overall chondrogenic differentiation, whereas $\mathrm{TXA}_{2}$ specifically increased chondrocyte hypertrophy. These data provide more insight in the function of the COXenzymes and prostaglandins during chondrogenic differentiation.

\section{Materials and Methods}

\subsection{Mouse growth plates}

The growth plates were isolated from tibias of 6 weeks old C57BL/6 mice (MUMC DEC approval 2008-042). The tibia's were isolated and fixated in formalin. The growth plates were separated from the rest of the tibia and decalcified in 0.5 M EDTA pH 7.8 for 2 weeks. EDTA was refreshed every two days. Growth plates were dehydrated and embedded in paraffin. Five micrometer sections were cut and positioned on Superfrost Plus slides for IHC.

\subsection{ATDC5 cell culture}

ATDC5 cells were cultured in a humidified atmosphere at $37^{\circ} \mathrm{C}, 5 \% \mathrm{CO}_{2}$. Proliferation medium (consisting of DMEM/F12 (Invitrogen, Carlsbad, CA, USA), 5\% FCS (PAA, Pasching, Austria), 1\% antibiotic/antimycotic (Invitrogen) and 1\% NEAA (Invitrogen)) was changed every two days. Differentiation medium comprised proliferation medium supplemented with $10 \mu \mathrm{g} / \mathrm{ml}$ insulin (Sigma, St. Louis, MO, USA), $10 \mu \mathrm{g} / \mathrm{ml}$ transferrin 
(Roche Applied Science, Indianapolis, IN, USA) and $30 \mathrm{nM}$ sodium selenite (Sigma, St. Louis, MO, USA). Cells were plated at 6400 cells $/ \mathrm{cm}^{2}$ in cell culture dishes, allowed to adhere overnight and the following day chondrogenic differentiation was initiated by changing the proliferation medium to differentiation medium. Differentiation medium was changed every two days (day 0-10) and daily (from day 10 on). To inhibit COX-1 activity SC-560 $(1 \mu \mathrm{M})($ Cayman Chemical, Ann Arbor, USA) or Mofezolac (5 $\mu \mathrm{M})$ (Sigma) were used and applied during the differentiation process. Similarly, COX-2 activity was inhibited by NS398 $(20 \mu \mathrm{M})$ (Cayman Chemical) or Celecoxib $(10 \mu \mathrm{M})$ (LC laboratories). $\mathrm{PGD}_{2}, \mathrm{PGE}_{2}, \mathrm{PGF}_{2 \alpha}$ and $\mathrm{U}-46619$ (a $\mathrm{TXA}_{2}$ receptor agonist, herein referred to as $\mathrm{TXA}_{2}$ ) (Cayman Chemical) were applied daily during the differentiation process at $0,0.1,1$ or 10 $\mu \mathrm{M}$ concentrations. These concentrations were selected in the range of the highest concentrations reported in the culture supernatants of control differentiated $t=14$ ATDC5 cells. $\mathrm{PGI}_{2}$ (from the same manufacturer) was not applied in these experiments as it is unstable in culture medium and therefore could be used in a reliable manner.

\subsection{Immunohistochemistry}

Growth plate sections of 6-weeks old mice were deparaffinized and antigen retrieval for COX-1 sections was performed by digestion with $0.4 \%$ hyaluronidase (Sigma) for 30 minutes at $37^{\circ} \mathrm{C}$. For $\mathrm{COX}-2$ detection, sections were incubated in boiling citrate buffer (1.8 $\mathrm{mM}$ citric acid and $8.2 \mathrm{mM}$ tri-sodium citrate) for 30 minutes. Endogenous peroxidase activity was inactivated and sections were blocked with 10\% normal sheep serum. Primary antibodies were: anti-COX-1 (Cayman Chemical, Ann Arbor, USA) and anti-COX-2 (BD Transduction Laboratories). After washing in PBS-T, bound antibodies were detected with HRP-labelled secondary antibodies (Dako, EnVision+ System-HRP labelled Polymer). Bound secondary antibodies were visualized by DAB substrate (Dako). Stained sections were counterstained with Mayer's Hematoxylin (Dako), dehydrated and mounted in Histomount (Thermo Shandon) for analysis.

\subsection{Prostaglandin measurement}

Prostaglandin concentration of $\mathrm{PGD}_{2}, \mathrm{PGE}_{2}, \mathrm{PGF}_{2 \alpha}$ and $\mathrm{TXB}_{2}$ (a stable metabolite of $\mathrm{TXA}_{2}$, herein referred to as $\mathrm{TXA}_{2}$ ) was determined in culture supernatants. Culture supernatants from different chondrogenic time points were stored in aliquots at $-80^{\circ} \mathrm{C}$ until analysis. Prostaglandin concentrations were determined by EIA's according to the manufacturers' protocol (Cayman Chemical). $\mathrm{PGI}_{2}$ concentrations could not be determined in a 
reliable manner by the EIA's from the same manufacturer and was therefore not included in these experiments.

\section{$\underline{2.5 \text { COX specificity assay }}$}

The colorimetrix COX inhibitor screening assay kit (Cayman Chemical) was used according to the manufacturers' protocol to determine COX isoform-specific inhibitory capacity for SC-560, Mofezolac, NS398 and Celecoxib.

\section{$\underline{2.6 R T-q P C R}$}

For RNA isolation, cells were washed 3 times with $0.9 \% \mathrm{NaCl}$ w/v and disrupted with 500 $\mu \mathrm{l}$ Trizol (Invitrogen). RNA isolation, RNA quantification by UV-spectrometry (Nanodrop, Thermo Scientific), and cDNA synthesis were performed as described before $^{1,2}$. Real time quantitative PCR (RT-qPCR) was performed using Mesagreen qPCR mastermix plus for SYBR ${ }^{\circledR}$ Green (Eurogentec, Seraing, Belgium). An Applied Biosystems ABI PRISM 7700 Sequence Detection System was used for amplification using the following protocol: initial denaturation at $95^{\circ} \mathrm{C}$ for 10 minutes, followed by 40 cycles of DNA amplification (denaturing for 15 seconds at $95^{\circ} \mathrm{C}$ and annealing for 1 minute at $60^{\circ} \mathrm{C}$ ) followed by a dissociation curve. Data were analysed using the standard curve method, mRNA expression was normalized to a reference gene ( $\beta$-actin) and gene expression was calculated as fold change as compared to day 0 ( $\mathrm{t}=0$ in graphs). Primer sequences are depicted in Table 6.1 .

\subsection{Immunoblotting}

Cells were washed three times with $0.9 \% \mathrm{NaCl}$ and lysed in RIPA buffer $(150 \mathrm{mM} \mathrm{NaCl}$, $1 \%$ NP-40, 0.5\% Sodium deoxycholate, 0.1\% SDS, $50 \mathrm{mM}$ Tris $\mathrm{pH}$ 8.0, 5.0 mM EDTA $\mathrm{pH}$ 8.0, $0.5 \mathrm{mM}$ dithiothreitol and $1 \mathrm{mM}$ phenylmethylsulfonylfluoride). Extracts were sonicated on ice using the Soniprep 150 (MSE, London, UK) at amplitude 10 for 14 cycles (1 second sonication and 1 second pause). Insoluble material was removed by centrifugation $\left(13,000 \mathrm{x} \mathrm{g}, 4^{\circ} \mathrm{C}\right)$. Protein concentration was determined using the $\mathrm{BCA}$ protein assay (Sigma). Polypeptides were separated by SDS-PAGE (samples were equally loaded) and subsequently transferred to nitrocellulose membranes by electroblotting. Primary antibodies for immunodetection were polyclonal goat anti-Col2a1 (Southern Biotech, Birmingham, AL, USA), polyclonal rabbit anti-Col10a1 (Calbiochem, Darmstadt, Germany), polyclonal rabbit anti-Sox9 (Abcam), mouse monoclonal anti-Runx2 (MBL, 
Woburn, USA) and mouse monoclonal anti- $\alpha$-Tubulin (Sigma, Saint Louis, MO, USA). Bound primary antibodies were detected with rabbit anti-goat, swine anti-rabbit or rabbit anti-mouse immunoglobulins conjugated with horseradish peroxidase (DakoCytomation, Glostrup, Denmark) and visualized by enhanced chemiluminescence. ECL signals were quantified using ImageJ 1.46f software, and relative differences, corrected for background and housekeeper, were determined as compared to control conditions.

Table 6.1: Primer sequences for RT-qPCR

\begin{tabular}{lll}
\hline Oligo sets mouse & Forward & $\underline{\text { Reverse }}$ \\
\hline Col2a1 & '5-TGGGTGTTCTATTTATTTATTGTCTTCCT-3' & '5-GCGTTGGACTCACACCAGTTAGT-3' \\
Col10a1 & '5-CATGCCTGATGGCTTCATAAA-3' & '5-AAGCAGACACGGGCATACCT-3' \\
Sox9 & '5-AGTACCCGCACCTGCACAAC-3' & '5-TACTTGTAGTCCGGGTGGTCTTTC-3' \\
Runx2 & '5-CGATGAAGACCCCAACCCTAA-3' & '5-ACTGGTAATGGCATCAAGGGATA-3' \\
COX-1 & '5-TCCTCACAGTGCGGTCCAA-3' & '5-AAGGCCTCCCAGCTGATGTAG-3' \\
COX-2 & '5-GGCCATGGAGTGGACTTAAA-3' & '5-AAGTGGTAACCGCTCAGGTG-3' \\
B-Actin & '5-GACAGGATGCAGAAGGAGATTACTG-3' & '5-CCACCGATCCACACAGAGTACTT-3' \\
\hline Oligo sets human & Forward & Reverse \\
\hline COL2A1 & '5-TGGGTGTTCTATTTATTTATTGTCTTCCT-3' & '5-GCGTTGGACTCACACCAGTTAGT-3' \\
COL10A1 & '5-ATGATGAATACACCAAAGGCTACCT-3' & '5-ACGCACACCTGGTCATTTTCTG-3' \\
SOX9 & '5-AGTACCCGCACCTGCACAAC-3' & '5-CGCTTCTCGCTCTCGTTCAG-3' \\
RUNX2 & '5-TGATGACACTGCCACCTCTTGA-3' & '5-GCACCTGCCTGGCTCTTCT-3' \\
COX-1 & '5-TCCTCACAGTGCGGTCCAA-3' & '5-AAGGCCTCCCAGCTGATGTAG-3' \\
COX-2 & '5-ACCAACATGATGTTTGCATTCTTT-3' & '5-GGTCCCCGCTTAAGATCTGTCT-3' \\
28S rRNA & '5-GCCATGGTAATCCTGCTCAGTAC-3' & '5-GCTCCTCAGCCAAGCACATAC-3'
\end{tabular}

The 5' -3 ' forward and reverse oligonucleotide sequences used for RT-qPCR are listed

\section{$\underline{2.8 \text { Cell proliferation }}$}

Cell proliferation was assessed by crystal-violet (Sigma, Saint Louis, MO, USA) staining. Cells were washed two times with $0.9 \% \mathrm{NaCl}$ and subsequently fixed with $4 \%$ paraformaldehyde in phosphate buffered saline for 10 minutes at room temperature. Fixed cells were washed 6 times with water and air dried for storage. Cells were incubated with $0.1 \%$ crystal-violet for 30 minutes at room temperature. Cells were washed six times with water to remove excess crystal-violet and allowed to air-dry in the dark overnight. Crystal-violet was extracted from the cells by incubation with $10 \%$ acetic acid for 15 minutes under continuous agitation. Extracted crystal-violet was determined spectrophotometrically at $590 \mathrm{~nm}$ using a plate reader (Biorad, Hemel Hempstead, UK). 


\subsection{Alcian blue staining}

GAG deposition was detected by alcian blue staining. Cells were washed two times with $0.9 \% \mathrm{NaCl}$ and subsequently fixed with $4 \%$ paraformaldehyde in phosphate buffered saline for 10 minutes at room temperature. Fixed cells were washed 6 times with water and air dried for storage. Fixed cells were incubated overnight with $1 \%$ alcian blue (Acros Organics, Geel, Belgium) in $0.1 \mathrm{M} \mathrm{HCl}$ at room temperature. Cells were washed six times with water to remove excess alcian blue and allowed to air-dry in the dark overnight. Alcian blue was extracted from the cells by incubation with guanidine- $\mathrm{HCl}(6 \mathrm{M})$ for 2 hours under continuous agitation. Extracted alcian blue was determined spectrophotometrically at $645 \mathrm{~nm}$ using a plate reader (Biorad, Hemel Hempstead, UK).

\section{$\underline{2.10 \text { Statistics }}$}

Statistical significance $(\mathrm{p}<0.05)$ was determined by two-tailed student t-tests using Graphpad PRISM 5.0 (La Jolla, CA, USA). To test for normal distribution of the input data, D'Agostino-Pearson omnibus normality tests were performed. All quantitative data sets presented passed the normality tests.

\section{Results}

\subsection{Expression of $C O X-1$ and $C O X-2$ during chondrogenic differentiation}

To examine the involvement of COX-1 and COX-2 during chondrogenic differentiation, spatiotemporal expression of these enzymes was determined in murine growth plates. As shown in Figure 6.1A, expression of COX-1 was specifically detected in chondrocytes located in the hypertrophic zone of the growth plate. COX-2 was expressed in chondrocytes in the hypertrophic zone of the growth plate, but also in the resting zone cells. To further study COX enzyme expression during chondrogenic differentiation, we analyzed expression of COX-1 and COX-2 during chondrogenic differentiation of ATDC5 cells. ATDC5 differentiation follows a well-defined chondrogenic program from chondroprogenitor to a hypertrophic chondrocyte ${ }^{1,21,22}$. Establishing chondrogenic differentiation of these cells, Col2a1 was increasingly expressed from day 7 onwards in ATDC5 differentiation (Figure 6.1B). Sox9 is expressed early in differentiation (first hours) and its expression increases again from day 7 onwards, together with Col2a1 (Figure 6.1B). 
A
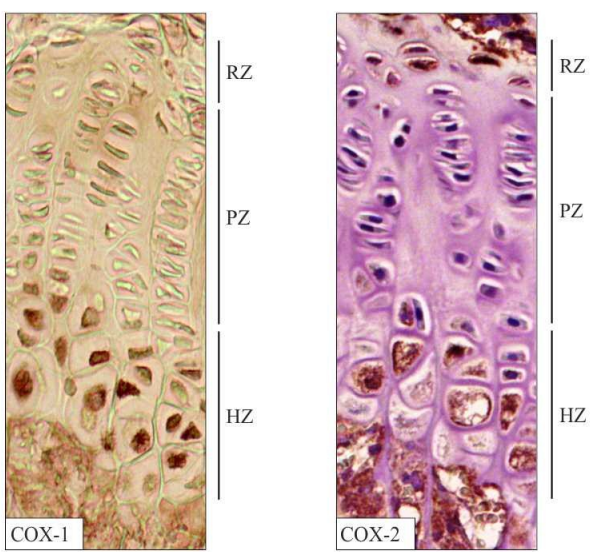

B

Col2al
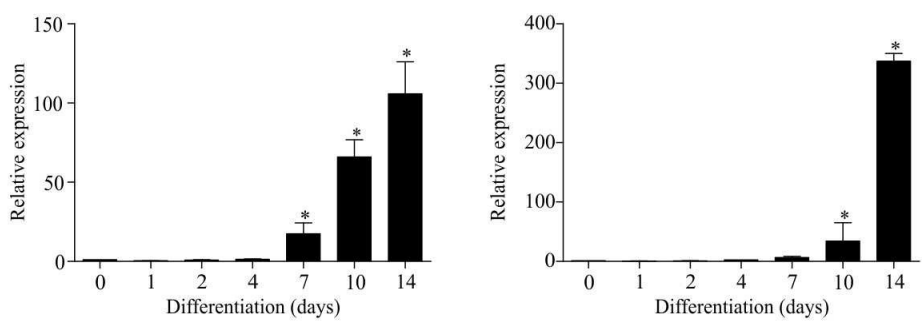

Sox 9

Runx2
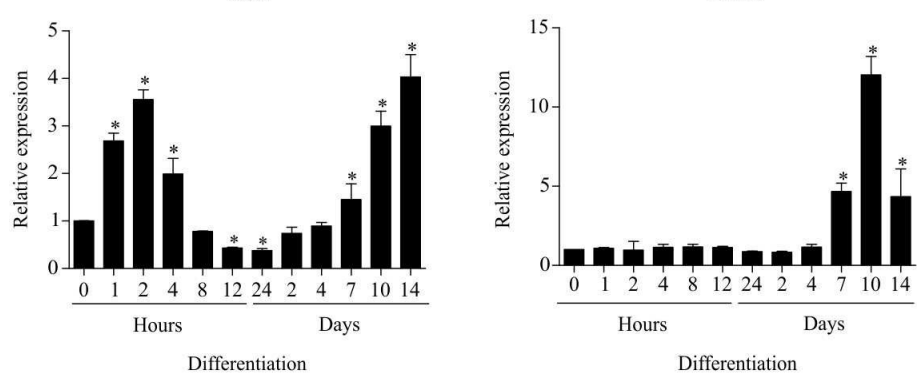

C

COX-1

COX-2
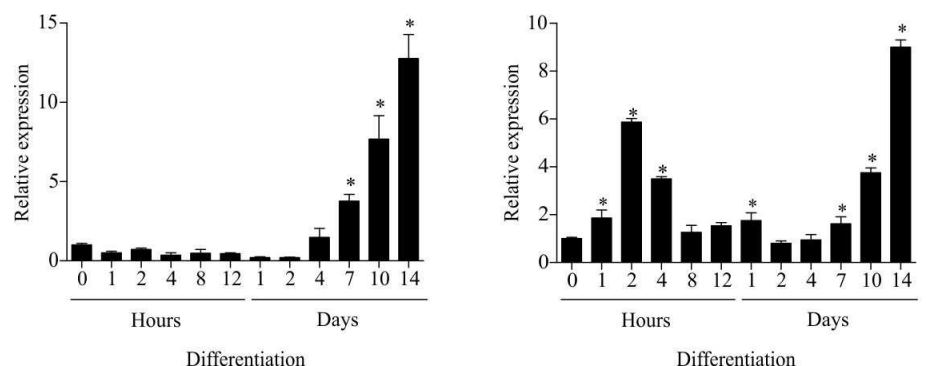
$\mathrm{D}$

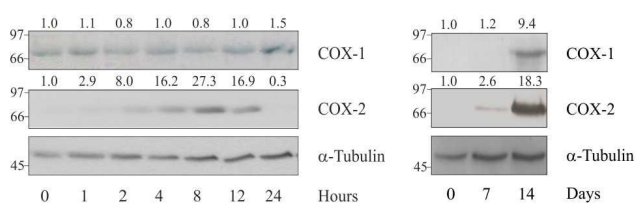

E

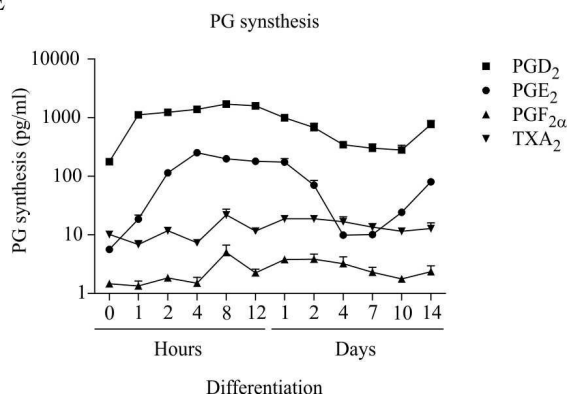

Figure 6.1: Expression of cyclooxygenases during chondrogenic differentiation from progenitor cells Involvement of COX-1 and COX-2 during chondrogenic differentiation was determined by assessing their expression patterns. (A). Spatiotemporal expression of COX-1 and COX-2 in 6 weeks old mouse growth plates was determined by IHC. RZ = resting zone, PZ = proliferative zone, HZ = hypertrophic zone. (B). Col2a1, Col10a1, Sox 9 and Runx 2 mRNA expression during chondrogenic differentiation of ATDC5 cells was determined by RT$\mathrm{qPCR}$ (relative to $\mathrm{t}=0$ and corrected for $\beta$-actin). (C). COX-1 and COX-2 mRNA expression during chondrogenic differentiation. In graphs, error bars represent mean \pm s.e.m.. * indicates $p<0.05$. (D). Protein expression of COX -1 and COX-2 at 0-24 hours (left panel) and at 0, 7 and 14 days in differentiation (right panel). Molecular weight markers $(\mathrm{kDa})$ are depicted on the left of immunoblots and relative quantifications are depicted on top of immunoblots. (E). Medium concentrations of $\mathrm{PGD}_{2}, \mathrm{PGE}_{2}, \mathrm{PGF}_{2 \alpha}$ and $\mathrm{TXA}_{2}$ during chondrogenic differentiation was determined by standard EIA.

Expression of chondrocyte hypertrophic genes Runx2 and Col10a1 increased from day 7 and day 10 onwards, respectively (Figure 6.1B). COX-1 expression was increased in ATDC5 differentiation from day 7 onwards (Figure 6.1C/D), correlating with the expression timing of Col2al and Col10al (Figure 6.1B). COX-2 expression was transiently upregulated in the first hours of chondrogenic differentiation and increased again from day 7 onwards (Figure 6.1C/D). These data correlate with the growth plate expression patterns of $\mathrm{COX}-1$ and $\mathrm{COX}-2$ and with our previous findings ${ }^{1,2}$. To functionally assess COX enzyme activity during chondrogenic differentiation, the concentration of the prostaglandins $\mathrm{PGD}_{2}, \mathrm{PGE}_{2}, \mathrm{PGF}_{2 \alpha}$ and $\mathrm{TXA}_{2}$ was determined in culture supernatants. Figure 6.1E shows that the concentration of $\mathrm{PGD}_{2}$ and $\mathrm{PGE}_{2}$ in the culture supernatants differs throughout differentiation and appears to be high in early differentiation and seems to increases late in differentiation, resembling the bi-phasic expression of COX-2. $\mathrm{PGF}_{2 \alpha}$ and $\mathrm{TXA}_{2}$ levels remain stably low during chondrogenic differentiation. 
3.2 COX-1 and COX-2 inhibition have differential effects on chondrogenic outcome and specific prostaglandin concentrations

A colorimetric inhibition assay using isolated ovine COX-1 or COX-2 was used to determine inhibitor concentrations at which specificity of the inhibitor for COX-2 over COX-1, or COX-1 over COX-2 was highest. This revealed optimal inhibitor concentrations for which similar inhibition capacities between the inhibitors were achieved, combined with the highest COX-specificity. For the COX-1 inhibitors these optimal concentrations were $1 \mu \mathrm{M}$ for SC-560 and $5 \mu \mathrm{M}$ for Mofezolac and for COX-2 inhibitors $20 \mu \mathrm{M}$ for NS398 and $10 \mu \mathrm{M}$ for Celecoxib (Figure 6.2). Using these concentrations, equal inhibition efficiency of COX-1 or COX-2 was achieved with as little as possible aspecificity for the other COX enzyme.

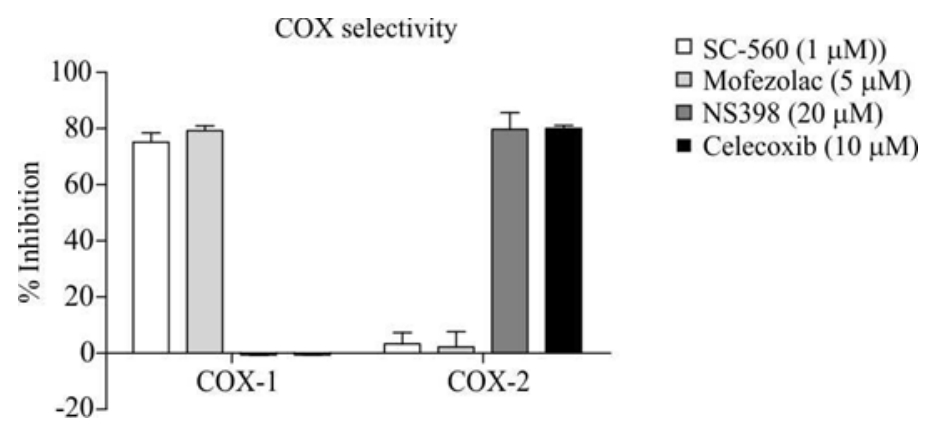

Figure 6.2: Specificity of $C O X-1$ and $C O X-2$ inhibitors

The selectivity of SC-560 and Mofezolac for COX-1 over COX-2 and selectivity of NS398 and Celecoxib for COX-2 over COX-1 was determined using a COX-1/COX-2 colorimetric inhibition assay. The inhibitory capacity was determined as "\% inhibition" compared to a maximum activity control condition. Bars represent average inhibition percentages of COX-1 and COX-2. Measurements were done in triplicate and error bars represent the variances in inhibitory activity.

To determine how chondrogenic differentiation of progenitor cells responds to COX-1 versus COX-2 inhibition, the different isoform-specific COX inhibitors were added (in concentrations as specified above and Figure 6.2) from 1) the start of differentiation and 2) from day 10 onwards (to determine whether a potential effect depends on the differentiation status of the chondrocyte). As compared to control conditions, inhibition of COX-1 by SC-560 or Mofezolac from the start of differentiation resulted in decreased Col2a1, Col10a1, Sox9 and Runx2 mRNA and protein expression at day 14 in differentia- 
tion (Figure 6.3A/B). Inhibition of COX-1 from day 10 onwards resulted in similar, although less pronounced effects on expression of these genes. Inhibition of COX-2, on the other hand, did not lead to decreased expression of Col2a1 and Sox9 at day 14 in differentiation and even increased Col2al expression (for Celecoxib at both time points and for NS398 when added from day 10 onwards in differentiation) (Figure 6.3A/B). A similar trend was detected for Sox9, although not significant. Inhibition of COX-2 did however affect the expression of chondrocyte hypertrophy genes Runx2 and Col10a1 at day 14 in differentiation, when COX-2 was inhibited from the start of the experiment ${ }^{2}$. Inhibition of COX-2 from day 10 onwards resulted in similar although less pronounced effects on these chondrocyte hypertrophy genes. COX-specific inhibition from the start of chondrogenic differentiation resulted in less increased GAG content for the COX-1 specific inhibitors at day 14 in differentiation, whereas COX-2 inhibitors did not affect GAG content (Figure 6.3C). Furthermore, cell proliferation capacity was assessed in these conditions. Except for SC-560, COX inhibitors did not alter cell proliferation capacity (Figure 6.3D).

In order to confirm successful COX-1 or COX-2 inhibition during chondrogenic differentiation and to determine whether both isoforms are equally involved in specific prostaglandin synthesis, culture supernatants from experiments shown in Figure 6.3 were analyzed for $\mathrm{PGE}_{2}, \mathrm{PGD}_{2}, \mathrm{PGF}_{2 \alpha}$ and $\mathrm{TXA}_{2}$ concentrations. As shown in Figure 6.4A (upper left panel), inhibition of COX-1 as well as COX-2 from day 0 or day 10 onwards caused a significant reduction in the $\mathrm{PGE}_{2}$ concentration at day 14 in differentiation, with the most efficient inhibition caused by both COX-1 inhibitors. COX-2 inhibition (both from day 0 and day 10 onward) by NS398 or Celecoxib also resulted in reduced PGF $_{2 \alpha}$ and $\mathrm{TXA}_{2}$ levels, but had no effect on $\mathrm{PGD}_{2}$ levels. Interestingly, COX-1 inhibition from the start of differentiation resulted in increased levels of $\mathrm{PGD}_{2}, \mathrm{PGF}_{2 \alpha}$ and $\mathrm{TXA}_{2}$ at day 14 in differentiation (Figure 6.4A). In contrast, COX-1 inhibition from day 10 in differentiation onwards, did not significantly influence $\mathrm{PGD}_{2}$ levels, but again increased $\mathrm{TXA}_{2}$ levels, while $\mathrm{PGF}_{2 \alpha}$ levels were now decreased as compared to control. Although the inhibitors enzymatically interfere with COX-activity, potential feedback mechanisms responding to diminished COX-activity might influence the level of COX-1 and COX-2 expression. To address this possibility we measured expression of COX-1 and COX-2 mRNAs. Inhibition of COX-1 by SC-560 or Mofezolac from the start of differentiation did not influence COX -1 mRNA expression at day 14 in differentiation, however when COX-1 was inhibited from day 10 onwards a slightly decrease in COX-1 mRNA expression was detected. Interestingly, COX-2 mRNA expression increased in all tested COX-1 inhibitory 
A
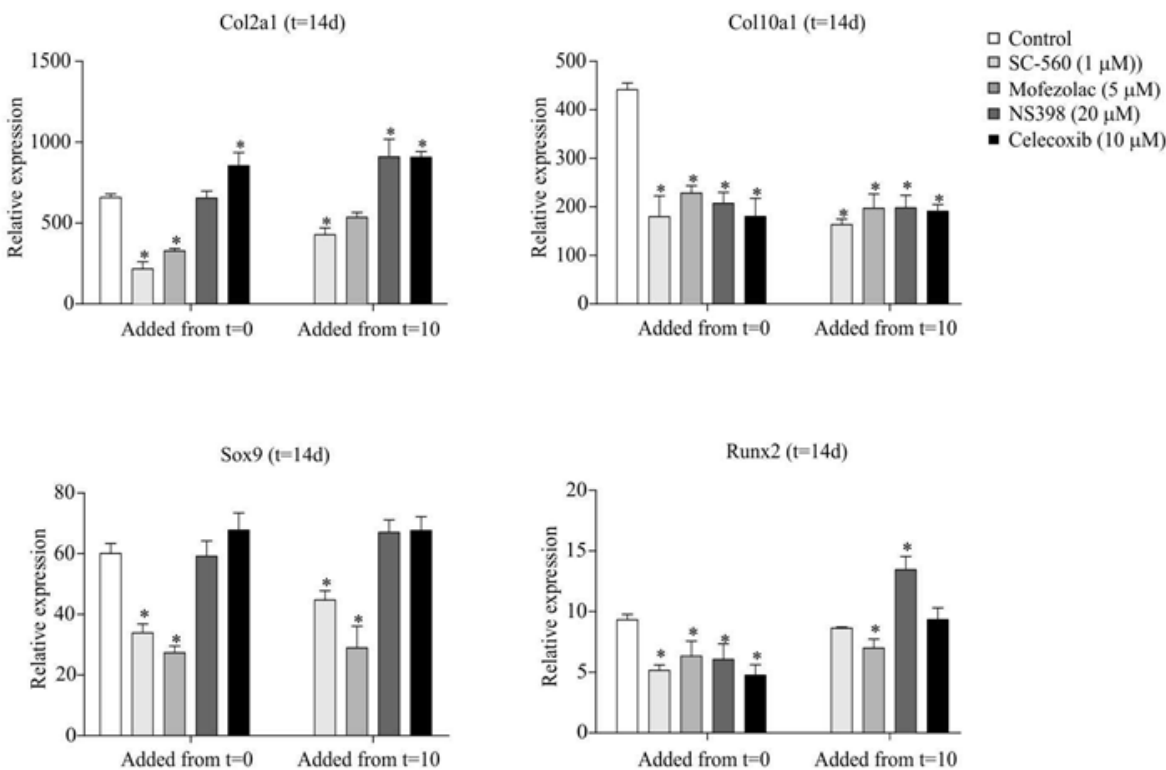

B
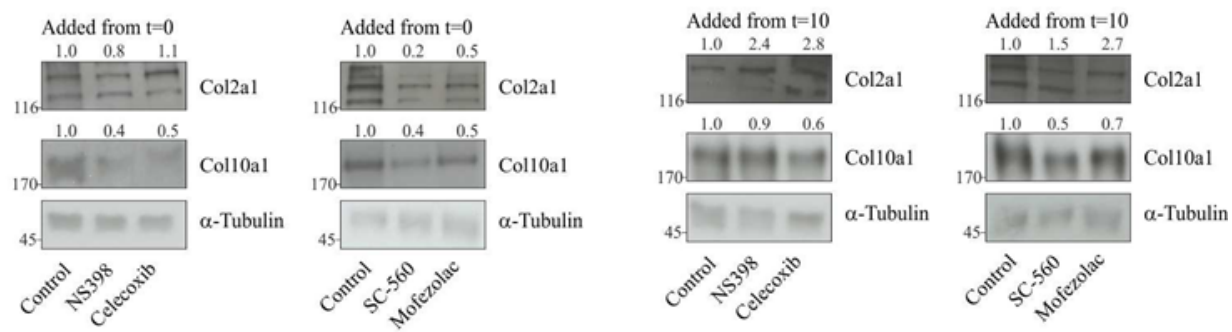

C

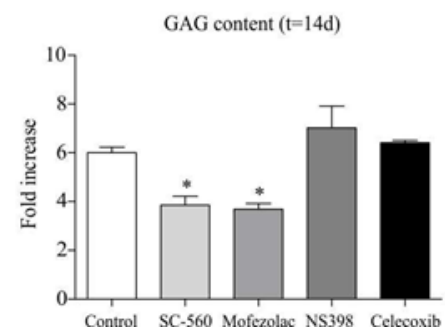

D

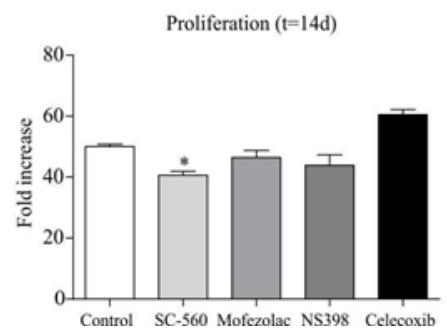


Figure 6.3: Effects of $C O X-1$ and $C O X-2$ specific inhibitors on chondrogenic differentiation of progenitor cells To determine the role the specific cyclooxygenases in chondrogenic differentiation specific COX-1 and COX-2 inhibitors were added to ATDC5 cells from the start of differentiation or from day 10 onwards. (A). Col2a1, Col10a1, Sox9 and Runx2 mRNA expression was determined at day 14 in differentiation by RT-qPCR (relative to $\mathrm{t}=0$ and corrected for $\beta$-actin). (B). Protein expression of Col2a1, Col10a1, Sox 9 and Runx 2 at day 14 in differentiation. Molecular weight markers $(\mathrm{kDa})$ are depicted on the left of immunoblots and relative quantifications are depicted on top of immunoblots. (C). Glycosaminoglycans (GAGs) were stained by alcian blue and fold change of $\mathrm{t}=14$ samples was calculated as compared to $\mathrm{t}=0$ samples. $(D)$. Differences in proliferation speed between conditions were determined by crystal-violet staining. Fold change (DNA) from samples from $\mathrm{t}=14$ was calculated relatively to day 0 . In graphs, error bars represent mean \pm s.e.m.. * indicates $p<0.05$.

conditions (Figure 6.4B). In cells treated with COX-2 specific inhibitors, COX-2 mRNA expression itself was increased as well, whereas COX-1 mRNA expression remained unchanged.

\section{$\underline{3.3 \text { Addition of specific prostaglandins during chondrogenic differentiation }}$}

As inhibition of COX-1 or COX-2 has strikingly different consequences on the chondrogenic outcome and appears to affect downstream specific prostaglandin synthesis differently, we tested whether these prostaglandins differently influence chondrogenic differentiation. To this end, increasing concentrations of prostaglandins $\mathrm{PGD}_{2}, \mathrm{PGE}_{2}, \mathrm{PGF}_{2 \alpha}$ and $\mathrm{TXA}_{2}$ were added to differentiating ATDC5. Addition of $\mathrm{PGE}_{2}$ and the highest concentration of $\mathrm{PGF}_{2 \alpha}$ caused a increased expression of Col2a1, Col10a1, Sox9 and Runx2 mRNA and protein at day 14 in differentiation (Figure 6.5A/B). TXA 2 addition specifically resulted in increased expression of the hypertrophic genes Col10a1 and Runx2 and had almost no effects on the expression of chondrogenic genes Col2a1 and Sox9. Expression of Sox9 and Runx 2 was increased by addition of $\mathrm{PGD}_{2}$ but this did only result in an increased trend in Col2a1 and Col10a1 expression (Figure 6.5A/B). Furthermore addition of the individual prostaglandins resulted in increased COX-1 and COX-2 mRNA expression (Figure $6.5 \mathrm{C})$.

Together, these data show that the specific prostaglandins have differential effects on chondrogenic differentiation of ADTC5 cells. $\mathrm{PGE}_{2}$ and $\mathrm{PGF}_{2 \alpha}$ showed the most increase in chondrogenic potential as determined by Col2a1, Sox9, Col10a1 and Runx2 expression, whereas $\mathrm{TXA}_{2}$ specifically resulted in increased Col10a1 and Runx2 expression; markers for chondrocyte hypertrophy. 
A
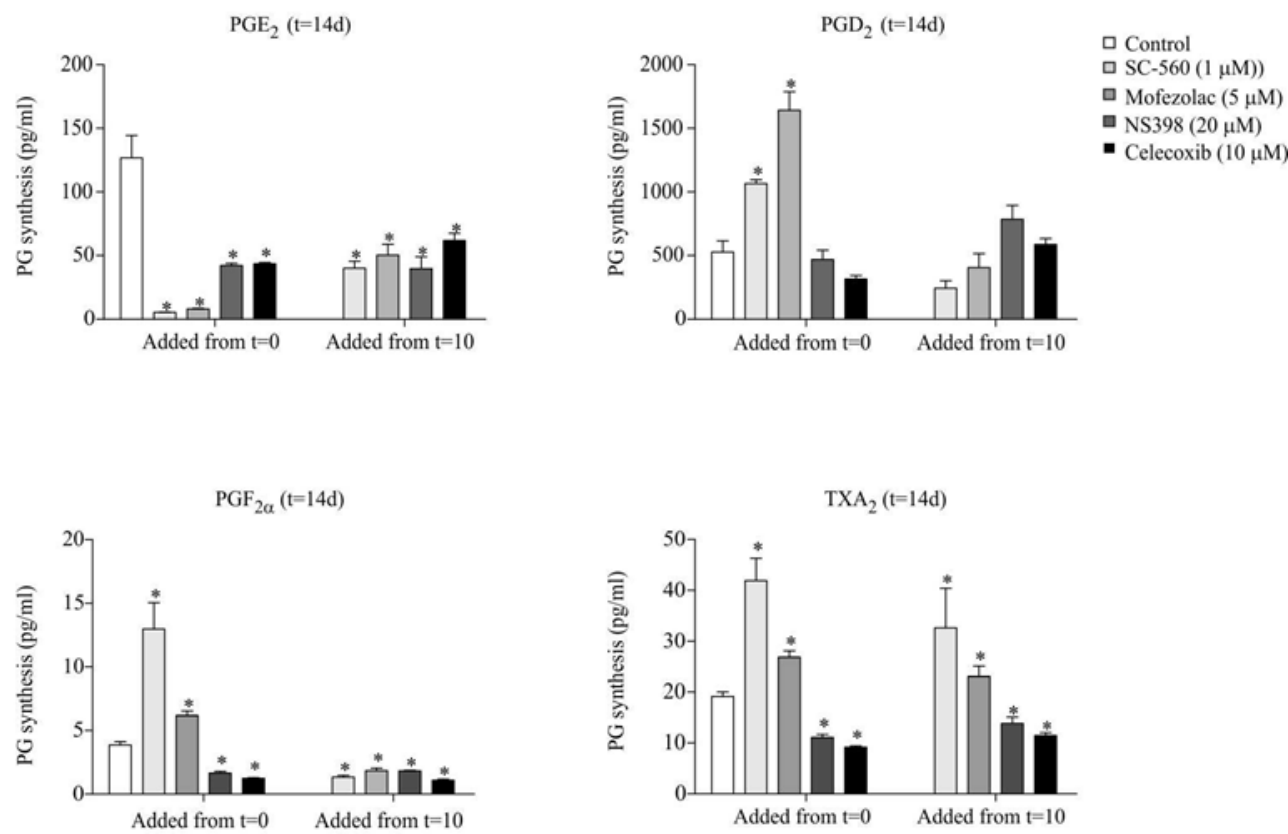

B
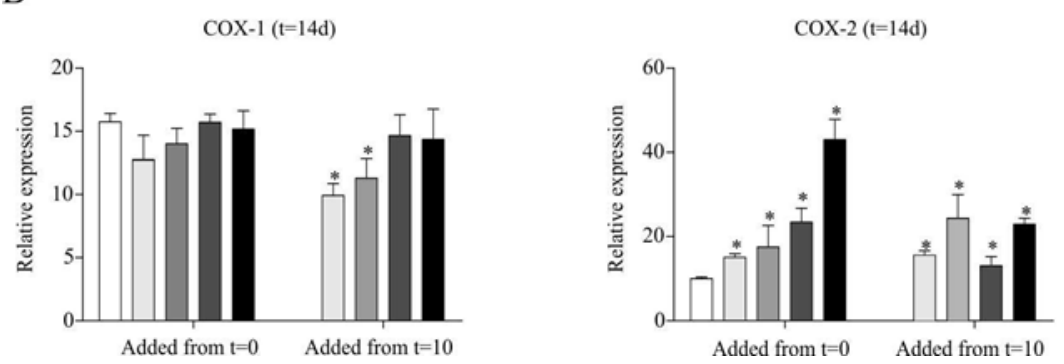

$\square$ Control

ㅁ SC-560 (1 $\mu \mathrm{M}))$

ㅁ Mofezolac $(5 \mu \mathrm{M})$

a $\operatorname{NS398}(20 \mu \mathrm{M})$

- Celecoxib $(10 \mu \mathrm{M})$

Figure 6.4: $C O X-1$ and $C O X-2$ specific inhibitors differentially influence prostaglandin levels during chondrogenic differentiation

Successful COX-1 or COX-2 inhibition by the inhibitors and possible differences in specific prostaglandin production was determined at day 14 in ATDC5 differentiation. NS398, Celecoxib, SC-50 or Mofezolac were added from the start of differentiation or from day 10 onwards. $(A)$. $\mathrm{PGE}_{2}, \mathrm{PGD}_{2}, \mathrm{PGF}_{2 \alpha}$ and $\mathrm{TXA}_{2}$ medium concentrations were determined at day 14 in differentiation by prostaglandin specific EIA. (B). COX-1 and COX-2 mRNA expression at day 14 in differentiation was determined by RT-qPCR (relative to $t=0$ and corrected for $\beta$-actin). In graphs, error bars represent mean \pm s.e.m.. * indicates $p<0.05$. 
A
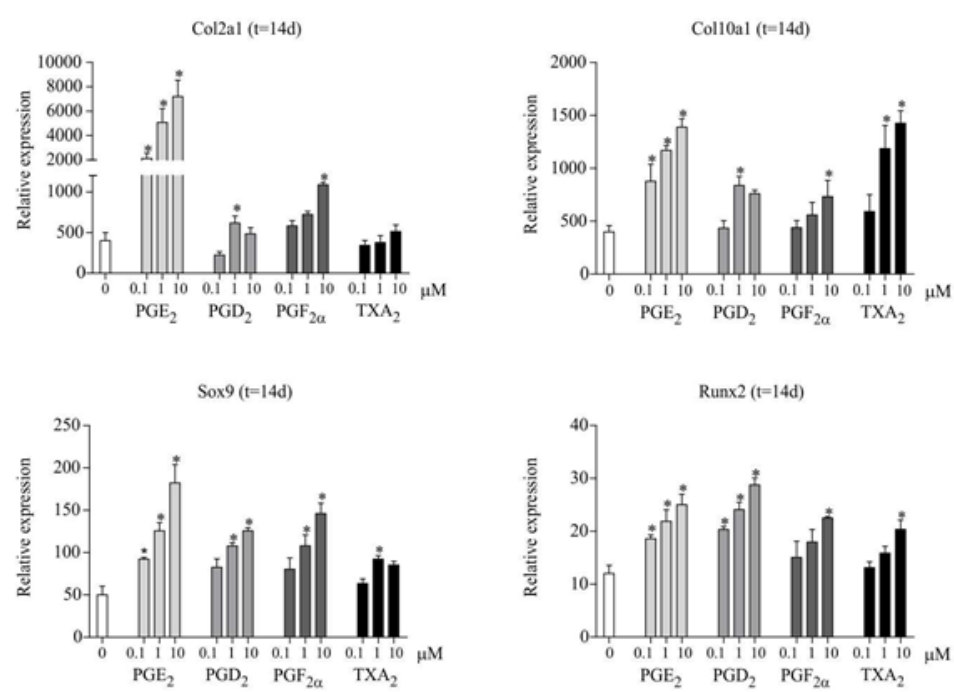

B
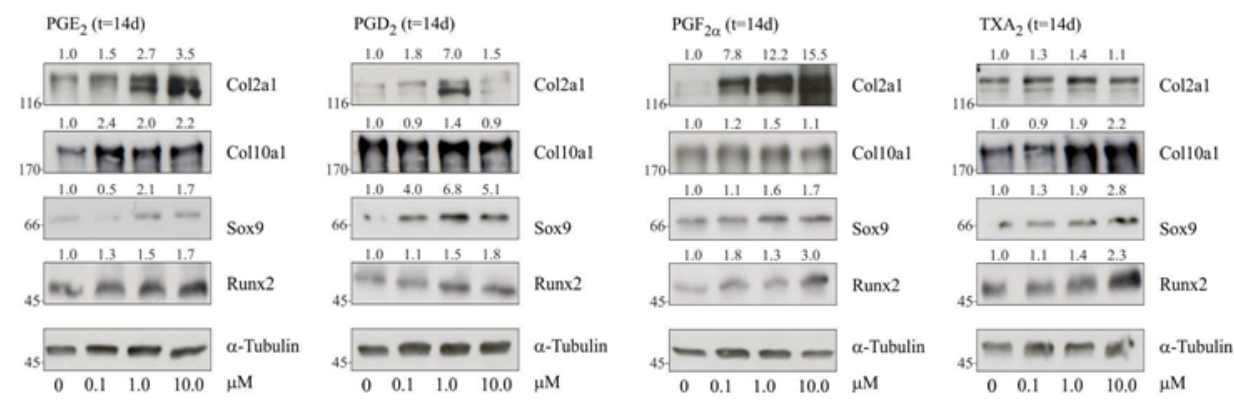

$\mathrm{C}$
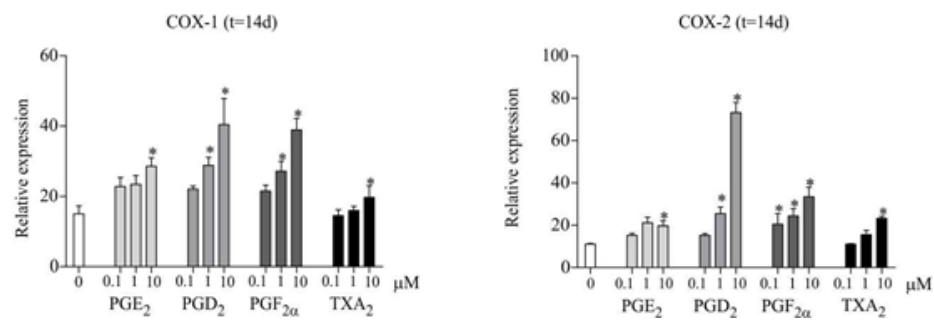

Figure 6.5: Prostaglandins differently regulate chondrogenic differentiation of progenitor cells

To test whether specific prostaglandins also have differential roles in chondrogenic differentiation, increasing concontrations $(0,0.1,1,10 \mu \mathrm{M})$ of $\mathrm{PGE}_{2}, \mathrm{PGD}_{2}, \mathrm{PGF}_{2 \alpha}, \mathrm{TXA}_{2}$ were added to differentiating ATDC5 cells. $(A)$. Col2a1, Col10a1, Sox9 and Runx2 mRNA expression was determined at day 14 in differentiation by RT-qPCR (relative to $\mathrm{t}=0$ and corrected for $\beta$-actin). (B). Protein expression of Col2a1, Col10a1, Sox9 and Runx2 at day 14 in differentiation. Molecular weight markers $(\mathrm{kDa})$ are depicted on the left of immunoblots and relative quantifications are depicted on top of immunoblots. (C). In samples from (A) mRNA expression of COX-1 and COX-2 was determined.In graphs, error bars represent mean \pm s.e.m.. $*$ indicates $\mathrm{p}<0.05$. 


\section{Discussion}

The majority of the studies reporting on the function of COX enzymes in cartilage and bone tissues focused on the function of $\mathrm{COX}-2$ and $\mathrm{PGE}_{2}$ in mature chondrocytes and osteoblasts $^{14-20}$. This leaves the role of COX-1 and other prostaglandins during chondrogenic differentiation from progenitor cells poorly studied.

In growth plates of 6 weeks-old mice we found that COX-1 and COX-2 presented differential temporospatial expression patterns; both isoforms were abundantly expressed in the hypertrophic chondrocytes of the growth plate, whereas specifically COX-2 was additionally found to be expressed in resting zone cells. These findings are in part in contrast with previous findings reported by Brochhaussen et al. ${ }^{14}$, who were the first to report COX expression in growth plates, and reported abundant expression of COX-1 throughout the rat growth plate and increased COX-2 expression in proliferative and hypertrophic zone of rat growth plates. Our results from murine growth plates showed interesting parallels with our in vitro ATDC5 observations though. Here COX-1 was only upregulated late in ATDC5 chondrogenic differentiation whereas COX-2 expression showed an initial peak during early differentiation and expression increased again from day 7 in differentiation and further. Recently, we and others have shown that NF- $\mathrm{B}$ signaling is essential for early chondrogenic differentiation and that early NF- $\kappa B$ activation is required for the transient expression of COX-2 during early differentiation ${ }^{1,23}$. Taken the above transient early COX-2 expression into account and the increased presence of COX-2 in the resting zone cells of murine growth plates, it is tempting to speculate on the role for COX-2 early in chondrogenic differentiation. However, based on expression of chondrogenic genes, we did not find any differences for COX-2 inhibition between day 0 and day 10 .

The partially distinct expression patterns for COX-1 and COX-2 may suggest differential involvement of the two COX isoforms in chondrogenic differentiation from progenitor cells. When COX-1 was specifically inhibited an overall decrease was found in induction of chondrogenic and hypertrophic genes as Col2a1, Sox9, Runx2 and Col10a1. Furthermore, chondrogenic gene expression responded similar when COX-1 was specifically inhibited late in differentiation, implying that COX-1 activity serves a crucial role during various phases of chondrogenic differentiation. These findings are supported by literature also showing adverse effects on proteoglycan synthesis and content when COX-1 was 
inhibited $^{24}$. Notably, in accordance with the overall chondrogenic inhibitory effect by specific COX-1 inhibitors, COX-1 is generally regarded to function as a "housekeeper", although the reason and mechanism behind the increased COX-1 expression during late/ hypertrophic differentiation remains to be elucidated ${ }^{7}$.

Inhibition of COX-2 activity from the start of differentiation specifically decreased expression of hypertrophic markers Runx2 and Col10a1, while leaving chondrogenic markers Col2a1, Sox9 and GAG content unchanged or even caused increased expression. These findings confirm a specific involvement of COX-2 over COX-1 in chondrocyte hypertrophy ${ }^{2}$. An unanswered question however remains how COX-2 can be specifically involved in regulating chondrocyte hypertrophy over COX-1, while the sole task of both is the synthesis of the $\mathrm{PGH}_{2}$ substrate for the synthesis of specific prostaglandins by dedicated downstream enzymes. A possible explanation might be found in the housekeeping function of COX-1 and that inhibiting COX-1 causes deleterious effects on the chondrocyte's $\mathrm{PGH}_{2}$ levels, while $\mathrm{PGH}_{2}$ synthesis by COX-2 might occupy only a small additional part of the cellular $\mathrm{PGH}_{2}$ pool. Alternatively, a differential involvement of COX-1 and COX-2 in the synthesis of specific prostaglandins could well be possible though, as we found that specific inhibition of COX-1 or COX-2 differently affected the excreted levels of specific prostaglandins in the culture supernatant. COX-1 inhibition showed decreased $\mathrm{PGE}_{2}$ concentration but no effects or an even increased concentration was seen for the other prostaglandins. COX-2 gene expression was found to be upregulated under influence of COX-1 inhibition and might explain the increased prostaglandin levels by redundancy mechanisms, although this would still not explain why $\mathrm{PGE}_{2}$ is specifically inhibited by COX-1 inhibition as opposed to the other measured prostaglandins. Inhibition of COX-2 resulted in decreased levels of all the prostaglandins but $\mathrm{PGD}_{2}$. Possible cellular rescue of COX expression under inhibitory conditions appears not to be mutually reciprocal, as opposed to above; COX-1 expression did not increase in the COX-2 inhibited conditions and would thus not provide an explanation for the inability for COX-2 inhibitors to down regulate $\mathrm{PGD}_{2}$ levels. Presently we do not have an explanation for this observation.

We demonstrated that $\mathrm{PGE}_{2}, \mathrm{PGF}_{2 \alpha}$ and in lesser extent also $\mathrm{PGD}_{2}$ have positive effects on chondrogenic and hypertrophic gene expression, whereas addition of $\mathrm{TXA}_{2}$ resulted in increased hypertrophic gene expression only. Some of these observations are in line with the report from Jacob et al., who also described a positive effect on chondrogenic outcome after addition of $\mathrm{PGD}_{2}$ and $\mathrm{PGF}_{2 \alpha}{ }^{25}$. However, $\mathrm{PGE}_{2}$ did not increase expression of chon- 
drogenic markers in this report, whereas in our study it resulted in the most pronounced effects from all tested prostaglandins. Differences of culture models; redifferentiating mature articular chondrocytes versus differentiating progenitor cells could be responsible for this discrepancy. In line with our results, several studies show that the addition of $\mathrm{PGE}_{2}$ result in increased cAMP levels and PKA signaling ${ }^{15,26}$ which led to increased mesenchymal progenitor cell chondrogenesis. Similarly, addition of $\mathrm{PGF}_{2 \alpha}$ resulted in an increase of chondrogenic marker expression in a rat chondrocyte cell line and mature chondrocytes $^{26,27}$.

Addition of $\mathrm{TXA}_{2}$, surprisingly, only increased hypertrophic marker expression. This nicely correlates with the effects of the COX-2 specific inhibitors, which resulted in decreased $\mathrm{TXA}_{2}$ expression and specific decreased hypertrophic gene expression. This possibly indicates a role for $\mathrm{COX}-2$ in $\mathrm{TXA}_{2}$ production and chondrocyte hypertrophy, which will be subject of further study. Another interesting observation is increased Sox 9 expression after $\mathrm{PGD}_{2}$ treatment, as it was previously described that $\mathrm{PGD}_{2}$ was able to induce Sox 9 transcriptional activation and subsequent differentiation of sertolli cells via its adenylcyclase-coupled DP1 receptor induced cAMP-dependent protein kinase A (PKA) signaling, which resulted in phosphorylation and nuclear translocation of Sox $9^{28}$. Although we investigated a different cell type in our study, one micromolar $\mathrm{PGD}_{2}$ resulted in a similarly increased Sox9 expression, which likely explains the subsequently increased Col2al expression. However, whether the action of $\mathrm{PGD}_{2}$ in chondrocytes is executed by the same signaling cascade needs further investigation.

The herein presented data clearly show that the various prostaglandins synthesized by differentiating progenitor cells may exert very different effects on the chondrogenic outcome. This may challenge the use of various non-steroidal anti-inflammatory drugs (NSAIDs) for progenitor cell-based cartilage regenerative medicine approaches, as slight differences in COX-selectivity between NSAIDs may lead to pronounced and unexpected differences in prostaglandin synthesis, which in turn will differentially affect the chondrogenic outcome of the differentiation process. This study demonstrates that much is yet to be learned on how specific prostaglandins influence chondrogenic differentiation and why inhibition of COX-1 and COX-2 enzymatic activity selectively influences prostaglandin concentrations. Future investigations may include a detailed analysis on the role of $\mathrm{TXA}_{2}$ in chondrocyte hypertrophy, as well experiments in which COX-1 or COX-2 activity is inhibited and in which the inhibition-phenotype is rescued by supplementation of selected prostaglandins. In addition, a potential role for prostacyclin $\left(\mathrm{PGI}_{2}\right)$ will be included in 
Chapter 6

further research. These latter approaches will be particularly interesting as it is expected to be able to further elucidate from which prostaglandin(s) a COX-inhibitory phenotype may depend.

\section{Acknouledgements}

This work is financially supported the Dutch Arthritis Association (grant LLP14) and the Dutch Stichting Annafonds|NOREF (grants 07/07 and 08/42). 
6. References

1. Caron MM, Emans PJ, Surtel DA, et al. Activation of NF-kappaB/p65 Facilitates Early Chondrogenic Differentiation during Endochondral Ossification. PLoS One 2012; 7:e33467.

2. Welting TJ, Caron MM, Emans PJ, et al. Inhibition of cyclooxygenase-2 impacts chondrocyte hypertrophic differentiation during endochondral ossification. European cells \& materials 2011;22:420-436; discussion 436-427.

3. Tsatsanis C, Androulidaki A, Venihaki M, et al. Signalling networks regulating cyclooxygenase-2. Int J Biochem Cell Biol 2006;38:1654-1661.

4. Van der Ouderaa FJ, Buytenhek M, Nugteren DH, et al. Purification and characterisation of prostaglandin endoperoxide synthetase from sheep vesicular glands. Biochim Biophys Acta 1977;487:315-331.

5. Gualde N, Harizi H. Prostanoids and their receptors that modulate dendritic cellmediated immunity. Immunol Cell Biol 2004;82:353-360.

6. Samuelsson B, Goldyne M, Granstrom E, et al. Prostaglandins and thromboxanes. Annu Rev Biochem 1978;47:997-1029.

7. Morita I. Distinct functions of COX-1 and COX-2. Prostaglandins Other Lipid Mediat 2002;68-69:165-175.

8. Kis B, Snipes JA, Gaspar T, et al. Cloning of cyclooxygenase-1b (putative COX3 ) in mouse. Inflamm Res 2006;55:274-278.

9. Chandrasekharan NV, Dai H, Roos KL, et al. COX-3, a cyclooxygenase-1 variant inhibited by acetaminophen and other analgesic/antipyretic drugs: cloning, structure, and expression. Proc Natl Acad Sci U S A 2002;99:13926-13931.

10. Warner TD, Mitchell JA. Cyclooxygenase-3 (COX-3): filling in the gaps toward a COX continuum? Proc Natl Acad Sci U S A 2002;99:13371-13373.

11. Simmons DL, Botting RM, Hla T. Cyclooxygenase isozymes: the biology of prostaglandin synthesis and inhibition. Pharmacol Rev 2004;56:387-437.

12. Needleman P, Turk J, Jakschik BA, et al. Arachidonic acid metabolism. Annu Rev Biochem 1986;55:69-102.

13. Vane JR, Bakhle YS, Botting RM. Cyclooxygenases 1 and 2. Annu Rev Pharmacol Toxicol 1998;38:97-120.

14. Brochhausen C, Neuland P, Kirkpatrick CJ, et al. Cyclooxygenases and prostaglandin E2 receptors in growth plate chondrocytes in vitro and in situ-prostaglandin E2 dependent proliferation of growth plate chondrocytes. Arthritis Res Ther 2006;8:R78.

15. Clark CA, Li TF, Kim KO, et al. Prostaglandin E2 inhibits BMP signaling and delays chondrocyte maturation. J Orthop Res 2009;27:785-792.

16. Di Battista JA, Dore S, Martel-Pelletier J, et al. Prostaglandin E2 stimulates incorporation of proline into collagenase digestible proteins in human articular chondrocytes: identification of an effector autocrine loop involving insulin-like growth factor I. Mol Cell Endocrinol 1996;123:27-35.

17. Mastbergen SC, Bijlsma JW, Lafeber FP. Synthesis and release of human cartilage matrix proteoglycans are differently regulated by nitric oxide and prostaglandin-E2. Ann Rheum Dis 2008;67:52-58.

18. Miyamoto M, Ito H, Mukai S, et al. Simultaneous stimulation of EP2 and EP4 is essential to the effect of prostaglandin E2 in chondrocyte differentiation. 
Osteoarthritis and cartilage / OARS, Osteoarthritis Research Society 2003;11:644652.

19. Mohamed-Ali H. Influence of interleukin-1 beta, tumour necrosis factor alpha and prostaglandin E2 on chondrogenesis and cartilage matrix breakdown in vitro. Rheumatol Int 1995;14:191-199.

20. Schwartz Z, Gilley RM, Sylvia VL, et al. The effect of prostaglandin E2 on costochondral chondrocyte differentiation is mediated by cyclic adenosine 3',5'monophosphate and protein kinase C. Endocrinology 1998;139:1825-1834.

21. Atsumi T, Miwa Y, Kimata K, et al. A chondrogenic cell line derived from a differentiating culture of AT805 teratocarcinoma cells. Cell Differ Dev 1990;30:109-116.

22. Chen L, Fink T, Zhang XY, et al. Quantitative transcriptional profiling of ATDC5 mouse progenitor cells during chondrogenesis. Differentiation 2005;73:350-363.

23. Itoh S, Saito T, Hirata M, et al. GSK-3alpha and GSK-3beta are involved in early stages of chondrocyte differentiation with functional redundancy through RelA phosphorylation. The Journal of biological chemistry 2012.

24. Mastbergen SC, Jansen NW, Bijlsma JW, et al. Differential direct effects of cyclo -oxygenase-1/2 inhibition on proteoglycan turnover of human osteoarthritic cartilage: an in vitro study. Arthritis Res Ther 2006;8:R2.

25. Jakob M, Demarteau O, Suetterlin R, et al. Chondrogenesis of expanded adult human articular chondrocytes is enhanced by specific prostaglandins. Rheumatology (Oxford) 2004;43:852-857.

26. Lowe $\mathrm{GN}, \mathrm{Fu} \mathrm{YH}, \mathrm{McDougall} \mathrm{S}$, et al. Effects of prostaglandins on deoxyribonucleic acid and aggrecan synthesis in the RCJ 3.1C5.18 chondrocyte cell line: role of second messengers. Endocrinology 1996;137:2208-2216.

27. Goldring MB, Suen LF, Yamin R, et al. Regulation of Collagen Gene Expression by Prostaglandins and Interleukin-1beta in Cultured Chondrocytes and Fibroblasts. Am J Ther 1996;3:9-16.

28. Malki S, Nef S, Notarnicola C, et al. Prostaglandin D2 induces nuclear import of the sex-determining factor SOX9 via its cAMP-PKA phosphorylation. Embo J 2005;24:1798-1809. 


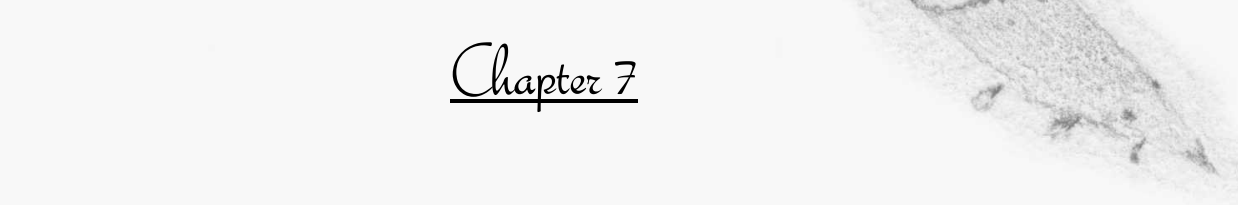

\section{Redifferentiation of Dedifferentiated Htuman Articular}

\section{Chondrocytes: Comparison of 2D and 3D Cultures}

\section{Adapted from:}

Redifferentiation of Dedifferentiated Human Articular Chondrocytes: Comparison of 2D and 3D Cultures

Marjolein M.J. Caron, Pieter J.Emans, Mariëlle M.E. Coolsen, Laura Voss, Don A.M. Surtel, Andy Cremers, Lodewijk W. van Rhijn and Tim J. M. Welting

Department of Orthopaedic Surgery, Maastricht University Medical Center, Maastricht, the Netherlands

Osteoarthritis and Cartilage. (2012); 20: 1170-1178 


\section{Alstract}

Introduction: Three-dimensional (3D) cultures are widely used to redifferentiate chondrocytes. However, the rationale behind the choice for 3D above two-dimensional (2D) cultures is poorly systematically investigated and mainly based on mRNA expression and glycosaminoglycan (GAG) content. The objective was to determine the differential redifferentiation characteristics of human articular chondrocytes (HACs) in monolayer, alginate beads and pellet culture by investigating mRNA expression, protein expression, GAG content and cell proliferation.

Methodology: Dedifferentiated HACs from six individuals were redifferentiated in identical medium conditions for 7 days in monolayer, alginate beads or pellet culture. Read -out parameters were expression of chondrogenic and hypertrophic mRNAs and proteins, GAG content and cell proliferation.

Results: 3D cultures specifically expressed chondrogenic mRNAs (collagen type II (COL2A1), SOX9, aggrecan (ACAN)), whereas 2D cultures did not. Hypertrophic mRNAs (collagen type X (COL10A1), RUNX2, MMP13, VEGF-A, osteopontin (OPN), ALP) were highly increased in 2D cultures and lower in 3D cultures. Collagen type I (COL1A1) mRNA expression was highest in 3D cultures. Protein expression supports most of the mRNA data, although an important discrepancy was found between mRNA and protein expression of COL2A1 and SOX9 in monolayer culture, stressing on the importance of protein expression analysis. GAG content was highest in 3D cultures, whereas chondrocyte proliferation was almost specific for 2D cultures.

Conclusions: For redifferentiation of dedifferentiated HACs, 3D cultures exhibit the most potent chondrogenic potential, whereas a hypertrophic phenotype is best achieved in $2 \mathrm{D}$ cultures. This is the first human study that systematically evaluates the differences between proliferation, GAG content, protein expression and mRNA expression of commonly used $2 \mathrm{D}$ and $3 \mathrm{D}$ chondrocyte culture techniques. 


\section{Introduction}

Articular cartilage basically comprises two fractions: a cellular fraction consisting of articular chondrocytes and an extracellular matrix (ECM) that is mainly composed of matrix associated water, collagens, glycosaminoglycans (GAGs) and other glycoproteins. The ECM determines the articular cartilage-specific functions as tensile strength combined with flexibility and resistance to compressive loads ${ }^{1-4}$.

Primary human articular chondrocytes (HACs) are enzymatically released from their cartilage matrix for autologous cell-based therapeutical interventions (e.g. Autologous Chondrocyte Transplantation $(\mathrm{ACT})^{5}$ ) as well as for research purposes ${ }^{3,6,7}$. However, long expansion time and multiple passaging, which are generally needed to acquire workable amounts of chondrocytes, lead to "dedifferentiation" of the isolated chondrocytes. Dedifferentiation of chondrocytes is defined as the gradual loss of molecular markers that define a differentiated chondrocyte. As dedifferentiation progresses, expression of ECM molecules as GAGs, collagen type II (COL2A1) and aggrecan (ACAN) is lost, whilst at the same time the cells acquire an increased fibroblastic phenotype, characterized by expression of collagen type I (COL1A1) $)^{8-10}$. In general chondrocytes are considered dedifferentiated after an average of about five monolayer passages ${ }^{8,11,12}$. Many investigations reported ways to decelerate the dedifferentiation process by supplementing the culture medium with several different growth factors ${ }^{13-17}$, varying with cell density ${ }^{18-21}$ or creating more "cartilage-like" culture conditions, such as hypoxic and hypertonic environments ${ }^{22,23}$. Other studies report the "redifferentiation" of dedifferentiated articular chondrocytes by specific culture conditions, resulting in regain of a differentiated chondrocyte phenotype and re-expression of cartilage marker molecules ${ }^{24-26}$. Generally, the cellular context is regarded as one of the most important contributors to redifferentiate articular chondrocytes. Conventional two-dimensional (2D) monolayer cultures are regarded as unsuitable, as monolayer expansion of articular chondrocytes does not support the chondrogenic phenotype during passaging. Instead, is well accepted that high cell density three-dimensional (3D) cultures favor the maintenance of the chondrocyte phenotype and support redifferentiation of dedifferentiated articular chondrocytes ${ }^{3}$. 3D cultures are performed by embedding chondrocytes in agarose ${ }^{27-29}$, fibrin glue gi-31 $^{27}$ alginate beads ${ }^{32-34}$, synthetic polymer gels or compressing into pellets by centrifugation ${ }^{12,16}$. Although 3D 
redifferentiation techniques are almost exclusively used instead of $2 \mathrm{D}$ approaches, comparative analyses between $2 \mathrm{D}$ and $3 \mathrm{D}$ redifferentiation approaches, to support the rationale behind the choice for a specific culture system, are hardly available. Also, although protein expression is the most valuable readout for functional gene expression, previous studies have hardly determined quantitative protein expression as a read-out parameter to compare the chondrogenic properties of different culture systems ${ }^{12,35,36}$.

The aim of this study was to compare the chondrogenic characteristics of commonly used 3D redifferentiation culture techniques for HACs (alginate beads and pellet culture) with a conventional 2D monolayer approach with high cell density. The chondrogenic capacities of these redifferentiation systems were compared at 7 days in differentiation based on gene expression of chondrogenic and hypertrophic/mineralization markers, as well as protein expression of a subset of these markers. Finally, GAG content and the proliferative capacity of redifferentiating chondrocytes were addressed in the selected culture systems.

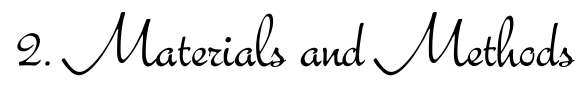

\subsection{HAC isolation and culture}

Chondrocytes were obtained from the unaffected regions of osteoarthritic (OA) cartilage from total knee arthroplasty (MEC approval 08-4-028). Cartilage was separated from the subchondral bone and cut into small pieces using a sterile surgical blade. Cartilage pieces were digested overnight at $37^{\circ} \mathrm{C}$ in collagenase type II solution $(300 \mathrm{U} / \mathrm{ml}$ in HEPES buffered DMEM/F12 (Invitrogen, Carlsbad, CA, USA) supplemented with antibiotics) under continuous agitation. The preparation was rinsed with $0.9 \% \mathrm{NaCl}$ over a $70 \mu \mathrm{m}$ cell strainer and plated in culture flasks. Cells were cultured in a humidified atmosphere at $37^{\circ} \mathrm{C}, 5 \% \mathrm{CO}_{2}$ and after reaching confluency the cells were continuously passaged 1:2 until passage 5. Culture medium consisted of DMEM/F12 (Invitrogen), 10\% FCS (PAA; Pasching, Austria), 1\% antibiotic/antimycotic (Invitrogen) and 1\% non-essential amino acids (NEAA; Invitrogen). Chondrogenic redifferentiation was performed with passage five cells from six individuals and each isolate was redifferentiated in monolayer, pellet and alginate culture. For monolayer the cells were plated 1day prior to start of differentiation at a density close to confluency $\left(30,000\right.$ cells $\left./ \mathrm{cm}^{2}\right)$ to mimic the high cell density known from $3 \mathrm{D}$ cultures. Chondrogenic differentiation was initiated by changing to 
differentiation medium (DMEM/F12, $1 \%$ antibiotic/antimycotic and $1 \%$ NEAA supplemented with $1 \%$ ITS (insulin-transferrin-sodium selenite media supplement,

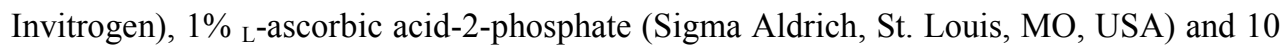
ng/ml TGF- $\beta 3$ (R\&D, Minneapolis, MN, USA)) ${ }^{13,14,37}$ For pellet culture redifferentiation, 300,000 cells per pellet were centrifuged at $120 \mathrm{xg}$ in conical tubes in differentiation medium (see above). The tubes were placed in a humidified atmosphere at $37^{\circ} \mathrm{C}, 5 \% \mathrm{CO}_{2}$ with the lid opened, after two days the pellets were transferred (three pellets per well) to uncoated 25-wells plates (Corning, Corning, NY, USA) and further allowed to differentiate. For alginate culture the cells were suspended in a sterile alginate solution $(1.2 \%$ alginate in $0.9 \% \mathrm{NaCl} ; 4,000,000$ cells $/ \mathrm{ml})$ and drops of this solution were carefully dispensed in a cold $\mathrm{CaCl}_{2}$ solution $(102 \mathrm{mM})$ using a 21 gauge needle. After ten minutes the alginate beads were washed in $0.9 \% \mathrm{NaCl}$ and transferred to standard six well culture dishes (10 beads per well) and cultured in differentiation medium. Differentiation medium was changed every two days for all three culture methods. Cells were harvested for RNA and protein analysis at day 7 in redifferentiation (see below).

\subsection{RT-qPCR}

For RNA isolation, monolayer cells and pellets were washed 3 times with $0.9 \% \mathrm{NaCl}$ and disrupted with $500 \mu 1$ Trizol. Alginate beads were first dissolved in ice cold citrate buffer, centrifuged and the cell pellet was then dissolved in Trizol (Invitrogen). RNA isolation, RNA quantification by UV-spectrometry (Nanodrop, Thermo Scientific), and cDNA synthesis were performed as described before ${ }^{38}$. Real time quantitative PCR (RT-qPCR) was performed using Mesagreen qPCR mastermix plus for SYBR ${ }^{\circledR}$ Green (Eurogentec, Seraing, Belgium). An Applied Biosystems ABI PRISM 7700 Sequence Detection System was used for amplification using the following protocol: initial denaturation at $95^{\circ} \mathrm{C}$ for 10 minutes, followed by 40 cycles of DNA amplification (denaturing for 15 seconds at $95^{\circ} \mathrm{C}$ and annealing for 1 minute at $60^{\circ} \mathrm{C}$ ) followed by a dissociation curve. Data were analyzed using the standard curve method, mRNA expression was normalized to $28 \mathrm{~S}$ rRNA as a housekeeping gene and gene expression was calculated as fold increase as compared to day 0 ( $\mathrm{t}=0$ in graphs). Primer sequences are depicted in Table 7.1.

\subsection{Immunoblotting}

Cells or pellets were washed three times with $0.9 \% \mathrm{NaCl}$ and lysed in RIPA buffer (150 $\mathrm{mM} \mathrm{NaCl}, 1 \% \mathrm{NP}-40,0.5 \%$ Sodium deoxycholate, $0.1 \%$ SDS, $50 \mathrm{mM}$ Tris $\mathrm{pH} 8.0,5.0$ 
mM EDTA pH 8.0, $0.5 \mathrm{mM}$ dithiothreitol and $1 \mathrm{mM}$ phenylmethylsulfonylfluoride). Alginate beads were first dissolved in ice cold citrate buffer, centrifuged and the cell pellet was then lysed in RIPA buffer. For all samples the same volume of RIPA buffer was used. Extracts were sonicated on ice using the Soniprep 150 (MSE, London, UK) at amplitude 10 for 14 cycles ( 1 second sonication and 1 second pause). Insoluble material was removed by centrifugation $\left(13,000 \mathrm{~g}, 4^{\circ} \mathrm{C}\right)$. Protein concentration was determined using the $\mathrm{BCA}$ protein assay (Sigma Aldrich). Polypeptides were separated by SDS-PAGE (samples were equally loaded) and subsequently transferred to nitrocellulose membranes by electroblotting. Primary antibodies for immunodetection were polyclonal goat antiCOL2A1 and polyclonal goat anti-COL1A1 (Southern Biotech, Birmingham, AL, USA), polyclonal rabbit anti-COL10A1 (Calbiochem, Darmstadt, Germany), polyclonal goat antiSOX9 (Abcam, Cambridge, UK), mouse monoclonal anti-RUNX2 (MBL, Woburn, MA, USA) and mouse monoclonal anti- $\alpha$-Tubulin (Sigma Aldrich, Saint Louis, MO, USA). Bound primary antibodies were detected with rabbit anti-goat, swine anti-rabbit or rabbit anti-mouse immunoglobulins conjugated with horseradish peroxidase (DakoCytomation, Glostrup, Denmark) and visualized by enhanced chemiluminescence (ECL). ECL signals were quantified using ImageJ $1.46 \mathrm{f}$ software.

\section{$\underline{2.4 D N A \text { quantification }}$}

DNA concentration in RIPA samples from day 0 and day 7 was determined using SYBR $®$ Green I Nucleic Acid stain (Invitrogen). A serially diluted standard curve of genomic control DNA (calf thymus, Invitrogen) in TE buffer $(10 \mathrm{mM}$ Tris/ $\mathrm{HCl} \mathrm{pH} 8.0,1 \mathrm{mM}$ EDTA) was included to quantify the DNA concentration in the samples. Before measurement, RIPA samples were diluted in TE buffer $(1 \mu 1$ RIPA sample and $99 \mu 1 \mathrm{TE}$ buffer) and standards were prepared ( $99 \mu 1$ standard in TE and $1 \mu 1$ RIPA buffer). SYBR ${ }^{\circledR}$ Green was diluted 10,000 times in TE buffer and $100 \mu$ of this solution was added to 100 $\mu \mathrm{l}$ of the above prepared samples or standards. Fluorescence was determined in standard 96 wells ELISA plates in a Spectramax M2 microplate reader (Molecular Devices): excitation $488 \mathrm{~nm}$ and emission $522 \mathrm{~nm}$. DNA concentration was determined using the standard curve and fold increase of the DNA content was calculated relatively to the average concentration value at day 0 in differentiation. 
Table 7.1: Primer sequences for RT-qPCR

\begin{tabular}{lll}
\hline Oligo sets human & Forward & Reverse \\
\hline COL1A1 & '5-TGTGCCACTCTGACTGGAAGA-3' & '5-AGACTTTGATGGCATCCAGGTT-3' \\
COL2A1 & '5-TGGGTGTTCTATTTATTTATTGTCTTCCT-3' & '5-GCGTTGGACTCACACCAGTTAGT-3' \\
COL10A1 & '5-ATGATGAATACACCAAAGGCTACCT-3' & '5-ACGCACACCTGGTCATTTTCTG-3' \\
ACAN & '5-GCAGCTGGGCGTTGTCA-3' & '5-TGAGTACAGGAGGCTTGAGGACT-3' \\
SOX9 & '5-AGTACCCGCACCTGCACAAC-3' & '5-CGCTTCTCGCTCTCGTTCAG-3' \\
RUNX2 & '5-TGATGACACTGCCACCTCTTGA-3' & '5-GCACCTGCCTGGCTCTTCT-3' \\
MMP13 & '5-CTTCACGATGGCATTGCTGAC-3' & '5-CGCCATGCTCCTTAATTCCA-3' \\
ALP & '5-GGGCTCCAGAAGCTCAACAC-3' & '5-GTGGAGCTGACCCTTGAGCAT-3' \\
VEGF-A & '5-ACGAAGTGGTGAAGTTCATGGAA-3' & '5-AAGATGTCCACCAAGGTCTCGAT-3' \\
OPN & '5-CGGTGCCTGACCCATCTC-3' & '5-TTTCTTCAGAGGACACAGCATTC-3' \\
28S rRNA & '5-GCCATGGTAATCCTGCTCAGTAC-3' & '5-GCTCCTCAGCCAAGCACATAC-3'
\end{tabular}

The 5' -3 ' forward and reverse oligonucleotide sequences used for RT-qPCR are listed

\subsection{Total GAG quantification}

Total sulfated GAG content was determined in RIPA samples from day 0 and day 7 cultures by using 1,9-dimethylmethylene blue (DMB; Polysciences). A standard curve of chondroitin sulfate (Sigma Aldrich) in PBS-EDTA (0.1 M Na $2 \mathrm{HPO}_{4}, 0.01 \mathrm{M} \mathrm{Na}_{2}$ EDTA, $\mathrm{pH}$ 6.5) was included to determine the GAG concentration in the samples. One hundred microliter diluted RIPA sample (5 $\mu 1$ RIPA sample and $95 \mu$ PBS-EDTA) or standard (95 $\mu \mathrm{l}$ standard and $5 \mu \mathrm{l}$ RIPA) was added to $200 \mu \mathrm{l} \mathrm{DMB}$ solution (46 $\mu \mathrm{M}$ DMB in $40 \mathrm{mM}$ $\mathrm{NaCl}, 0.4 \mathrm{M}$ glycine $\mathrm{pH} 3.0$ ), and the extinctions were determined spectrophotometrically at both $595 \mathrm{~nm}$ and $540 \mathrm{~nm}{ }^{39,40}$. GAG content was determined using the generated standard curve, corrected for DNA content (see above) and expressed as $\mu \mathrm{g}$ GAG/ng DNA.

\subsection{Statistics}

Statistical significance $(\mathrm{p}<0.05)$ was determined by two-tailed student $\mathrm{t}$-tests (paired for Figures 7.1 and 7.3, unpaired with Welch's correction for Figure 7.2D) using Graphpad PRISM 5.0 (La Jolla, CA, USA). To test for normal distribution of the input data, D'Agostino Pearson omnibus normality tests were performed. All quantitative data sets presented here passed the normality tests. Error bars in graphs represent mean $\pm 95 \%$ confidence interval. In the dot plots p-values are presented for every statistical calculation. 
3. Results

\section{$\underline{3.1 \text { Gene expression during chondrogenic redifferentiation in } 2 D \text { and } 3 D \text { culture }}$}

Dedifferentiated HACs were redifferentiated in $2 \mathrm{D}$ monolayer ( $\mathrm{n}=6$ individuals) or in $3 \mathrm{D}$ alginate beads ( $\mathrm{n}=6$ individuals) or pellet cultures ( $\mathrm{n}=6$ individuals) for 7 days under identical medium conditions. To assess the level of chondrogenic redifferentiation, induction of COL2A1, ACAN and SOX9 mRNA expression was determined compared to $\mathrm{t}=0$ (Figure $7.1 \mathrm{~A})^{41}$. Gene expression data reveal the induction of COL2A1 and ACAN mRNAs in both 3D systems. In monolayer redifferentiation however, the expression of these markers was not induced as compared to $t=0$. Although not significantly different, induction of COL2A1 and ACAN was slightly higher in alginate beads than in pellets. In line with the data above, no SOX9 mRNA induction was detected in monolayerredifferentiated chondrocytes. In contrast, alginate beads and pellet culture supported the induction of SOX9, although the alginate beads supported a much higher induction of SOX9 compared to pellet culture redifferentiation.

Redifferentiation of HACs might induce expression of hypertrophic and mineralization markers $^{41}$. As shown in Figure 7.1B, COL10A1, RUNX2 and ALP mRNAs were highly induced in monolayer cultures after 7 days. Although significantly lower than in monolayer, COL10A1 and RUNX2 induction was also found in both 3D cultures. Expression of MMP13, VEGF-A and osteopontin (OPN) differed from the other above hypertrophic/mineralization markers. MMP13 induction was found in monolayer and pellet culture, but was hardly induced in alginate beads. In contrast, induction of VEGF-A was found to be the highest in pellet culture and for OPN similar induction characteristics were found. In 2D cultures COL1A1 is only marginally induced as was the case for pellet cultures (Figure 7.1C). COL1A1 expression was substantially higher in alginate beads, indicating fibrocartilaginous redifferentiation in this culture system.

Overall the gene expression data indicate that redifferentiation of dedifferentiated HACs in $2 \mathrm{D}$ and $3 \mathrm{D}$ culture systems results in different redifferentiated chondrocyte phenotypes from which the 3D systems support chondrogenic differentiation the best, whereas the $2 \mathrm{D}$ monolayer system gives rise to induction of hypertrophic/mineralization markers. 
A

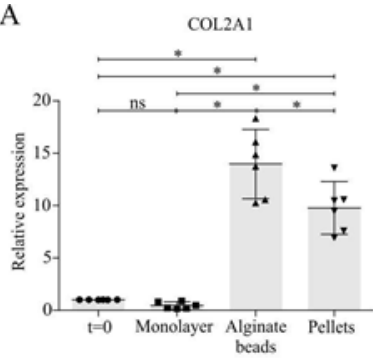

B

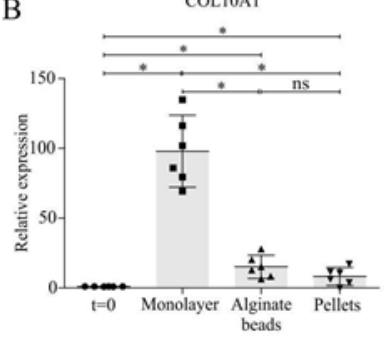

VEGFA

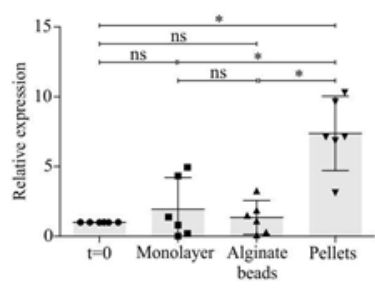

C

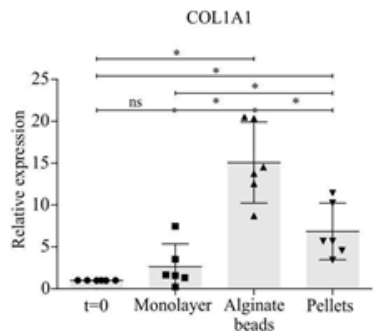

soxe

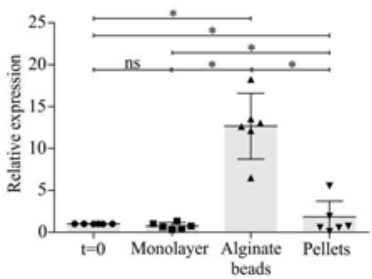

RUNX2
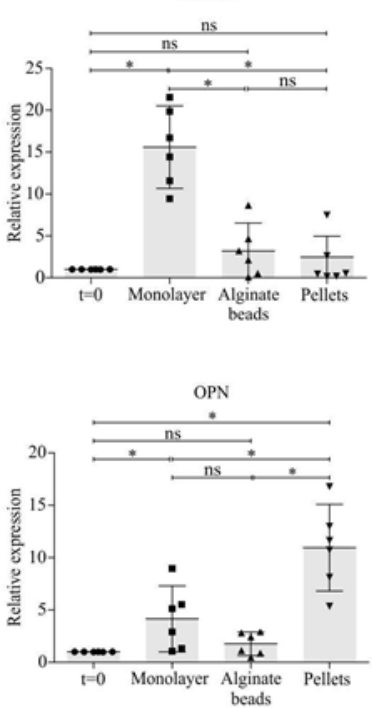
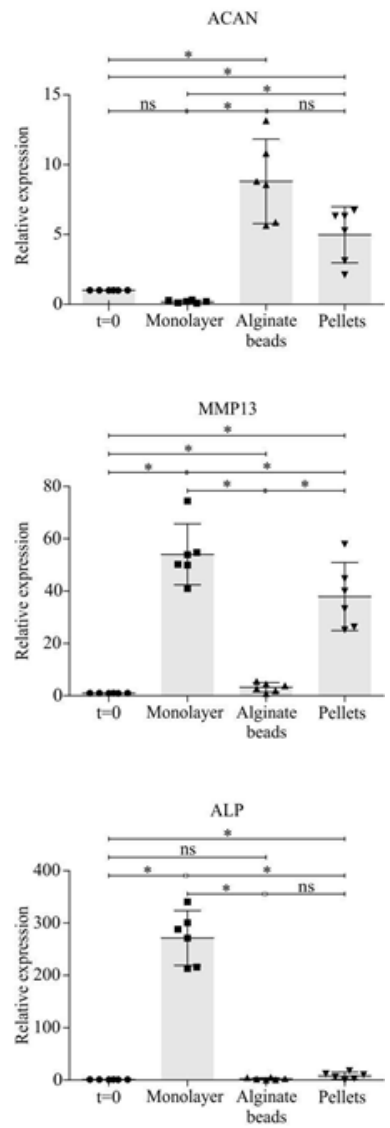

Figure 7.1: RT-qPCR analysis of dedifferentiated HACs after redifferentiation in $2 D$ and $3 D$ culture systems

Dedifferentiated HACs ( $\mathrm{n}=6$ individuals) were redifferentiated in 2D monolayer or 3D alginate beads or pellet culture using identical medium conditions. Relative induction of gene expression was determined at day 7 in redifferentiation as compared to day 0 . (A). Induction of mRNAs of the chondrogenic genes COL2A1, SOX9 and ACAN was determined. $(B)$. Similar to A. but here relative induction of hypertrophic/mineralization markers COL10A1, RUNX2, MMP13, VEGF-A, OPN and ALP was determined. (C). Similar to A. but now the fibrotic marker COL1A1 was determined. In all graphs dots represent the average value of three individual samples per HAC isolate ( $n=6$ individual donors) and error bars represent mean $\pm 95 \%$ confidence interval. Statistical significant $\mathrm{p}$-values $(\mathrm{p}<0.05)$ are shown by an $*$, ns $=$ not statistical significant. 


\subsection{Protein expression during chondrogenic redifferentiation in $2 D$ and $3 D$ culture}

In general, the majority of previously published work on redifferentiation of dedifferentiated chondrocytes uses mRNA expression and GAG content as the major determinant to investigate chondrogenic redifferentiation of HACs. However, it is well known that protein expression not always faithfully follows mRNA expression. Taking this fact into account we concluded that investigating mRNA expression alone does not provide enough information to truly compare the characteristics of the herein tested $2 \mathrm{D}$ and 3D culture systems for chondrogenic redifferentiation of HACs. Therefore, COL2A1, SOX9, COL1A1, COL10A1 and RUNX2 protein expression was measured in similar samples as described above. The results of the immunoblot analyses are shown in Figure 7.2A (monolayer), Figure 7.2B (alginate beads) and Figure 7.2C (pellets). Quantitative data of immunoblots are presented in Figure 7.2D.

In remarkable contrast to the mRNA expression data (see above), COL2A1 protein expression was found to be induced in all three culture systems. Interestingly, induction of COL2A1 protein expression in monolayer culture was found to be equally high as in alginate beads and higher as in pellet cultures. Induction of SOX9 protein expression was also found in all three culture systems with no significant differences between the different redifferentiation protocols, which is again in sharp contrast with mRNA expression data. In line with the gene expression analyses of hypertrophic markers above, COL10A1 and RUNX2 protein expression was induced in all systems, with monolayer presenting a significantly higher induction of COL10A1 and RUNX2 protein expression as compared to the $3 \mathrm{D}$ culture systems. Finally, induction of COL1A1 protein expression was also found in all three systems, revealing significantly higher COL1A1 expression levels in alginate beads and pellet cultures as compared to monolayer. Generally these protein expression data confirm the gene expression data that were derived from the different redifferentiation methods, albeit $2 \mathrm{D}$ redifferentiation provided some interesting contradictions when gene expression was compared to protein expression.

\section{$\underline{3.3 \text { Total GAG content and cell proliferation during chondrogenic redifferentiation in } 2 D}$} and $3 D$ cultures

Besides expression of chondrogenic transcription factors and extracellular proteins, GAG synthesis is an important hallmark of chondrogenic differentiation. As can be seen in Figure 7.3A, monolayer redifferentiation did not support the synthesis of GAGs more than was already synthesized by the dedifferentiated chondrocytes at $\mathrm{t}=0$. Both $3 \mathrm{D}$ systems 
A

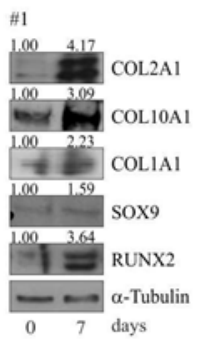

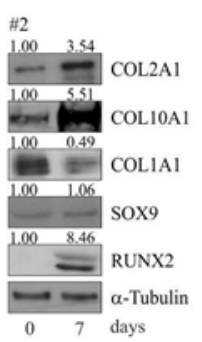

B
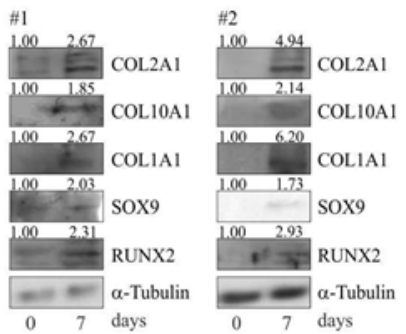

C
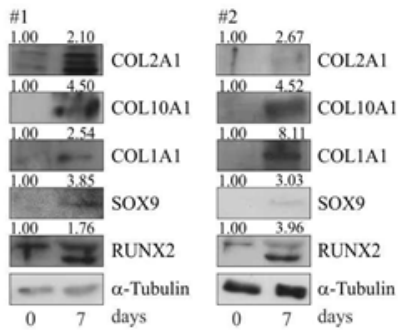

Monolayer
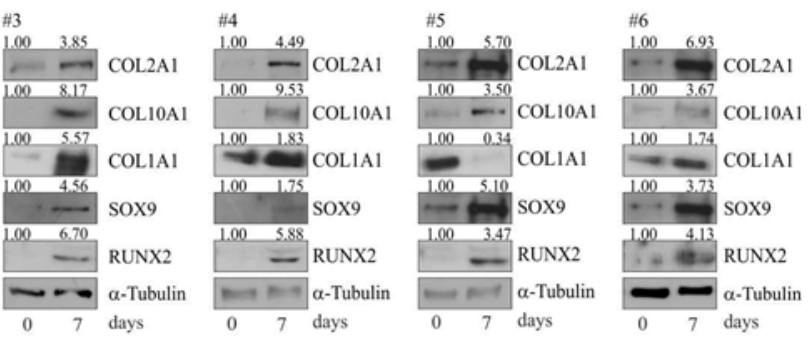

\section{Alginate beads}
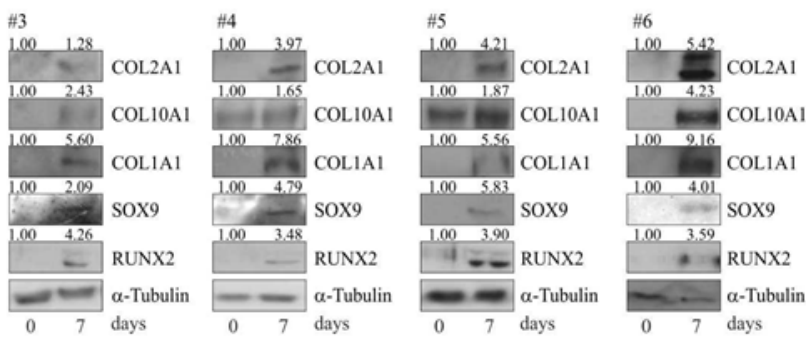

Pellets
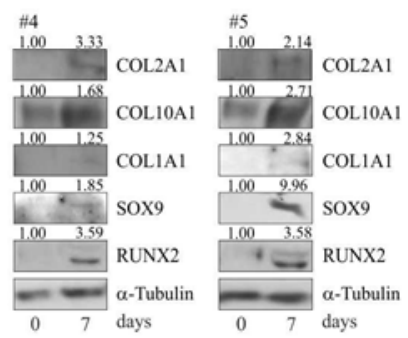

D
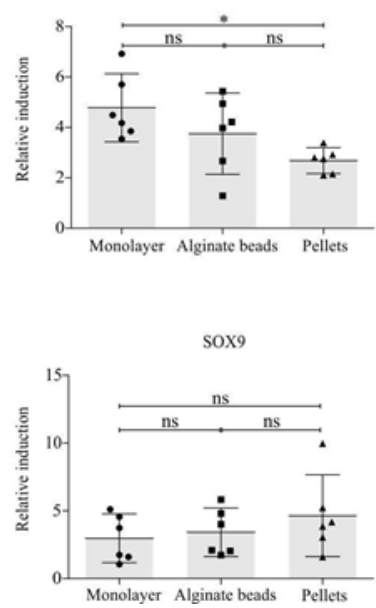

COL10AI
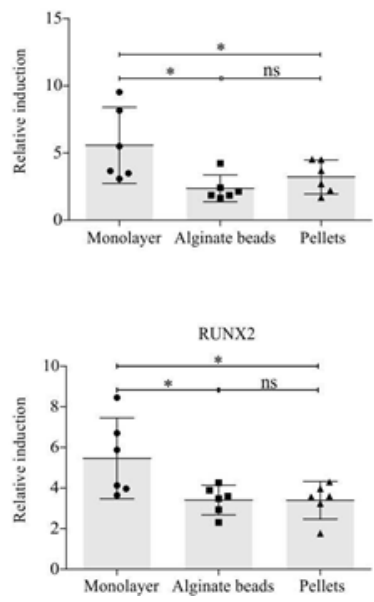

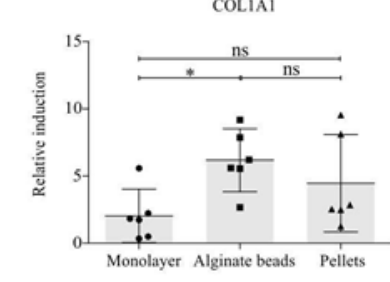

COLIAI 
Figure 7.2: Immunoblot analysis of dedifferentiated HACs after redifferentiation in $2 D$ and $3 D$ culture systems HACs ( $n=6$ individuals) were redifferentiated in monolayer, alginate beads or pellet culture. Protein expression of a subset of the genes from Fig. 7.1 (COL2A1, COL10A1, COL1A1, SOX9, RUNX2) was determined at day 7 in redifferentiation as compared to day 0. $(A)$. Protein expression of HACs redifferentiated in 2D monolayer culture. $(B)$. Protein expression of HACs redifferentiated in 3D alginate beads. $(C)$. Protein expression of HACs redifferentiated in 3D pellet cultures. $\alpha$-Tubulin was used as housekeeper. Quantifications of ECL signals (corrected for the complementary $\alpha$-Tubulin signals and relatively to $\mathrm{t}=0$ ) are depicted on top of all immunoblots. In immunoblots COL2A1 runs at $120 \mathrm{kDa}$; COL10A1 at $180 \mathrm{kDa}$; COL1A1 at $130 \mathrm{kDa}$; SOX9 at $60 \mathrm{kDa}$; RUNX2 at $66 \mathrm{kDa}$ (lower band). (D). Graphs of immunoblot quantifications from (A) to (C). Each dots represent the determined value for each of the individual HAC isolates $(n=6)$ and error bars represent mean $\pm 95 \%$ confidence interval. Statistical significant $\mathrm{p}$-values $(\mathrm{p}<0.05)$ are shown by an $*$, ns $=$ not statistical significant.

A

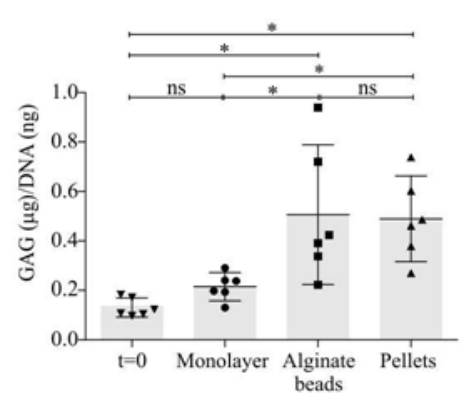

B

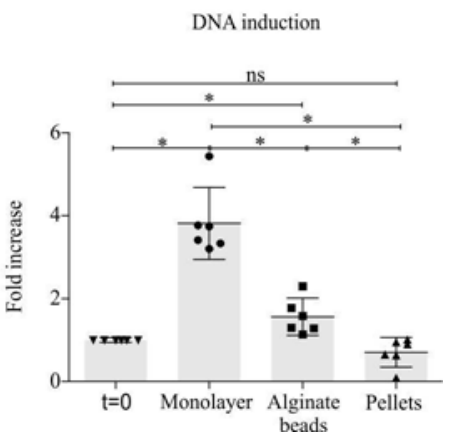

Figure 7.3: GAG content and DNA induction in redifferentiated HACs

Dedifferentiated HACs ( $\mathrm{n}=6$ individuals) were redifferentiated in monolayer, alginate beads or pellet culture, using identical medium conditions. $(A)$. Total GAG content was determined in day 0 and day 7 samples. To correct for cells numbers, GAG content was normalized to total DNA. (B). The DNA content used in (A). to correct GAG content is shown to determine cell proliferation. DNA induction was calculated relative to DNA content in day 0 samples. In all graphs dots represent the average value of three individual samples per HAC isolate $(n=6$ individual donors). Statistics were calculated for these $n=6$ and error bars represent mean $\pm 95 \%$ confidence interval. Statistical significant $\mathrm{p}$-values $(\mathrm{p}<0.05)$ are shown by an $*$, ns $=$ not statistical significant.

however allowed increased GAG content per cell as a result of the redifferentiation process. Notably, although seeded at close to $100 \%$ confluency, the monolayer redifferentiation culture allowed an almost 4 -fold increase in cell numbers (determined by DNA content; Figure 7.3B), whereas cell numbers did hardly (alginate) or not (pellet) increase in both 3D systems. Taken together, these data show that not all culture systems supported the synthesis of GAGs and differentially allow cell proliferation. 


\section{Discussion}

It is well established that expansion of isolated articular chondrocytes using conventional monolayer passaging leads to loss of the chondrogenic phenotype and results in an overall fibroblast-like phenotype $\mathrm{e}^{3,8-10}$. This phenomenon is called dedifferentiation and for cartilage regenerative applications and chondrocyte studies this adverse phenomenon is avoided by using the isolated chondrocytes in relatively early passages (when cells are not (fully) dedifferentiated yet) or induce redifferentiation by means of selected medium supplements (e.g. ascorbic acid, TGF- $\beta$ isoforms, insulin, transferrin, selenite ${ }^{13,14,37,42}$ and others). Both methods are generally combined with $3 \mathrm{D}$ culture approaches that mimic the cartilage environment ${ }^{3}$. As $2 \mathrm{D}$ monolayer passaging does not support maintenance of the chondrogenic phenotype, this culture method is generally regarded as unsuitable for redifferentiation. Bernstein and colleagues compared the redifferentiation characteristics of dedifferentiated porcine chondrocytes in different culture models ${ }^{43}$. However, to the best of our knowledge no study reported on the comparison between 2D and 3D culture methods for the redifferentiation of human dedifferentiated chondrocytes. Thereby our study contributes to the understanding how these culture methods differentially determine the outcome of in vitro chondrogenic redifferentiation and may provide a rational behind the choice for one or the other culture system when addressing different research questions. As strikingly little evidence is available in the literature supporting human chondrocyte redifferentiation on the protein level ${ }^{35}$, an important additional aspect of our study is the inclusion of immunoblot detection to determine expression of important chondrogenic marker molecules. Overall these protein expression data support the results from mRNA expression analyses, however show some remarkable contradicting results specifically for the monolayer redifferentiation cultures.

In general, 3D chondrocyte culture methods allow the cells to differentiate for a very long time (generally between 14 and 28 days). Our primary focus was to address the chondrogenic capacity of the three herein selected culture methods. As terminal chondrocyte differentiation is more likely to progress at late timepoints in differentiation we decided to allow differentiation for 7 days only. Indeed, both 3D culture methods showed mRNA induction of chondrogenic markers such as COL2A1, ACAN and SOX9, whereas gene induction of hypertrophic markers (COL10A1, ALP, RUNX2) remained relatively low. This is in sharp contrast with monolayer redifferentiation in which we 
found a very high induction of the above mentioned hypertrophic markers, associated with little chondrogenic capacity as concluded from COL2A1, ACAN and SOX9 mRNA induction. Also, in time points between $\mathrm{t}=0$ and $\mathrm{t}=7$ did these chondrogenic markers not increase in expression, excluding the possibility of "missing" a potential expression peak of these chondrogenic genes (Figure 7.4). The chondrocyte hypertrophic genes COL10A1 and RUNX2 did increase in expression over time. These finding suggests that monolayer redifferentiation supports a more chondrocyte hypertrophic phenotype as compared to $3 \mathrm{D}$ culture conditions.

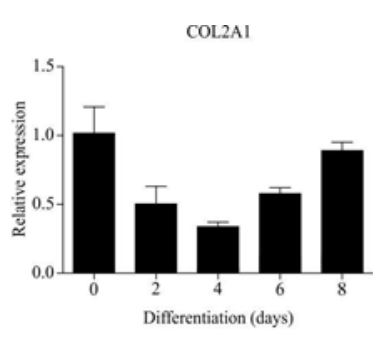

COL10A1

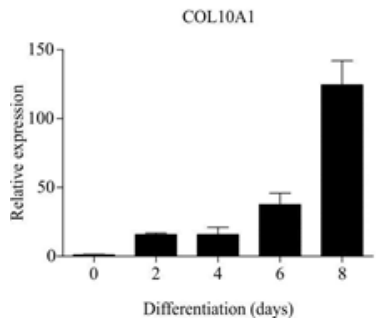

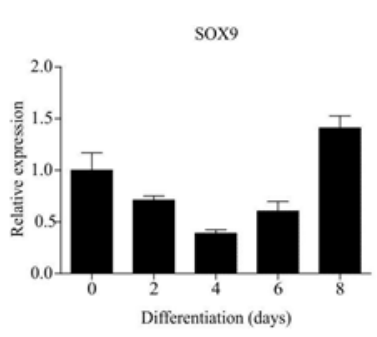

RUNX2

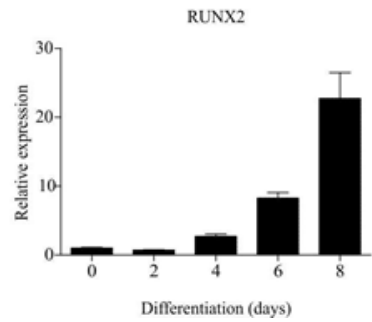

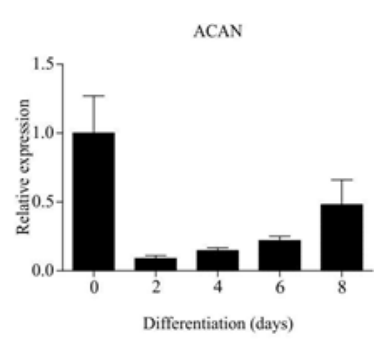

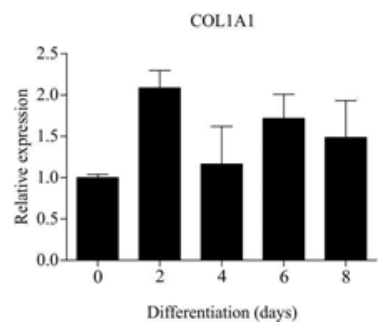

Figure 7.4: RT-qPCR analysis of dedifferentiated HACs after redifferentiation in monolayer over time

Dedifferentiated HACs ( $\mathrm{n}=3$ individuals) were redifferentiated in $2 \mathrm{D}$ monolayer. Relative induction of gene expression of COL2A1, SOX9, ACAN, COL10A1, RUNX2 and COL1A1 was determined at day 2, 4, 6 and 8 in redifferentiation as compared to day 0 . In all graphs bars represent the average value of three individual samples per HAC isolate ( $\mathrm{n}=3$ individual donors) and error bars represent mean $\pm 95 \%$ confidence interval.

Protein expression analysis of hypertrophic markers COL10A1 and RUNX2 supports the gene expression data from all three culture methods. For chondrogenic markers, protein expression data from alginate and pellet culture support the results of their respective gene expression analyses. However, monolayer-redifferentiated chondrocytes show contradicting results between mRNA and protein expression specifically for COL2A1 and SOX9 induction. A similar observation has previously been described for COL2 $\mathrm{A}^{36}$. 
Whereas COL2A1 and SOX9 mRNA expression is not or hardly induced in monolayer, COL2A1 and SOX9 proteins are expressed in monolayer culture. Notably, the magnitude of COL2A1 and SOX9 protein induction in monolayer was not significantly different from alginate or pellet culture. In general, discrepancies between mRNA and protein expression can be the result of posttranscriptional regulation of the mRNA, as well as differences in mRNA and protein turnover rates ${ }^{44}$. Mechanistically, induction of protein expression can be the result of two different pathways. Firstly, induction of mRNA expression and subsequently translation of this mRNA into functional protein. The other mechanism omits the induction of mRNA expression, but instead only translates the cellular pool of the given mRNA into protein. This latter mechanism might be dominant in the monolayer redifferentiation cultures. A possible explanation why this was specifically observed in monolayer might be found in the high hypertrophic nature of monolayer redifferentiation, which is not likely to support (SOX9 dependent) chondrogenic redifferentiation. Under these hypertrophic conditions, SOX9 and COL2A1 mRNA may not be transcribed de novo, but a remainder pool of SOX9 and COL2A1 mRNAs in the dedifferentiated HACs could still be translated. Indeed we found that SOX9 and COL2A1 mRNAs are still detectable in dedifferentiated HACs (data not shown). However, currently we cannot explain how the induction of SOX9 protein in monolayer does not lead to induction of COL2A1 mRNA. A dysfunctional nuclear translocation of SOX9 could explain this phenomenon $^{45,46}$.

Nevertheless, although COL2A1 and SOX9 proteins were equally induced in all three culture models, the alginate and pellet cultures showed the lowest levels of COL10A1 and RUNX2 protein induction and at the same time the highest induction of total GAG synthesis. This supports the conclusions drawn from the mRNA expression data discussed above and of previous work $^{47}$, indicating that $3 \mathrm{D}$ chondrocyte culture displays a high chondrogenic potential, whereas we here additionally show that monolayer is inducing a more chondrocyte hypertrophic phenotype in these cells. A mechanism behind the predominantly monolayer-associated hypertrophic phenotype remains to be determined. Reoxygenation of hypoxic proliferating growth plate chondrocytes from the subchondral compartment is one of the mechanisms partaking in growth plate chondrocyte hypertrophic differentiation $^{48,49}$. Also in vitro there is ample evidence that oxygen tension is a major determining factor in chondrocyte hypertrophic differentiation ${ }^{48,50} .3 \mathrm{D}$ chondrocyte cultures may have limited oxygen availability due to limitations in oxygen transport/diffusion throughout the $3 \mathrm{D}$ structure, whereas monolayer cultures may have the highest oxygen 
availability of the three herein tested culture systems. Consequently, this might provide an explanation for the monolayer-associated hypertrophic phenotype.

With regard to the other hypertrophy-associated markers (MMP13, VEGF-A and OPN ${ }^{41}$ ) some interesting observations were made that are not completely in line with the above hypertrophic COL10A1, RUNX2 and ALP mRNA expression data. MMP13, OPN and VEGFA mRNAs are not only expressed in monolayer but are also remarkably high induced in pellet cultures, which in contrast with the almost absence of COL10A1, RUNX2 and ALP mRNA induction in pellet cultures. The pellet cultures induced COL2A1, SOX9 and ACAN mRNAs to a lesser extent than alginate; this could possibly relate to the relative high induction of MMP13, VEGF-A and OPN mRNAs in this culture system.

Another interesting finding is that the induction of COL1A1 mRNA and protein, associated with a more fibrocartilaginous phenotype, was the highest in alginate cultures. In contrast, monolayer-redifferentiated chondrocytes were found to be the lowest inducers of COL1A1 mRNA and protein. This is in contrast with the findings by Bernstein and colleagues $^{43}$, who found that redifferentiation of dedifferentiated porcine chondrocytes in monolayer resulted in relatively high COL1A1 mRNA levels as compared to other culture systems. Possibly the osteoarthritic nature of our cell source may be a factor in the fibrotic outcome of our alginate cultures ${ }^{51}$. It remains however a remarkable observation that this was specifically found for the alginate cultures that, in general, seem to support the highest chondrogenic capacity during redifferentiation. It is therefore likely that differences in technical procedures between our study and previous work ${ }^{43}$ may provide a basis for the observed differences in experimental results; different alginate types were used as well as different compositions of the chondrogenic culture medium.

As a crucial component of the redifferentiation medium we supplemented our cultures with $10 \mathrm{ng} / \mathrm{ml}$ TGF- $\beta 3$. Previous research reported that the use of one or the other TGF- $\beta$ isoform (TGF- $\beta 1,-2,-3$ ) has no differential influence on the outcome of the chondrogenic differentiation of human mesenchymal progenitor cells ${ }^{52,53}$. However Cals et al. reported that in the presence of mineralizing medium supplements (e.g. $\beta$-glycerophosphate), TGF- $\beta 3$ gives rise to a more mineralized cartilage phenotype, as compared to other TGF- $\beta$ isoforms ${ }^{53}$. Although we did not add such mineralizing components, the specific use of TGF- $\beta 3$ could explain the relative high induction of mineralization markers in all three culture systems. However, it remains unclear why monolayer culture specifically provokes the highest expression of these markers. 
In agreement with previous studies ${ }^{34}$, we found that cell numbers increased the highest in the $2 \mathrm{D}$ monolayer redifferentiation cultures, even though the differentiation medium was deprived from serum and the cells were seeded at close to $100 \%$ confluency. Both $3 \mathrm{D}$ cultures showed no such high increase in cell numbers. Physical limitations of the 3D cultures to support cell proliferation could explain the absence of increase in DNA content, as opposed to monolayer culture. Interestingly, as the appearance of clonal populations of proliferating chondrocytes is a hallmark of early osteoarthritis ${ }^{54-56}$, this predominantly monolayer-specific property might add to preferable characteristics for a cell culture model to investigate osteoarthritis-associated processes.

In summary, we compared the redifferentiation characteristics of dedifferentiated HACs in three different cell models (monolayer, alginate beads and pellet culture). In addition to commonly used mRNA expression analysis, for the first time we herein report protein expression analyses to compare the redifferentiation characteristics of chondrocytes. Overall these protein data support gene expression data, although an important discrepancy was found between mRNA and protein expression of COL2A1 and SOX9 in monolayer culture, stressing on the importance of protein expression analyses in this culture system. Furthermore, both 3D cultures were found to exhibit the highest chondrogenic capacity, whereas monolayer redifferentiation appears to result in expression of hypertrophic and mineralization markers and as such this model might be used for complementary studies addressing hypertrophic differentiation of chondrocytes.

\section{Acknouledgments}

This work is financially supported the Dutch Arthritis Association (grant LLP14) and the Dutch Stichting Annafonds|NOREF (grants 07/07 and 08/42). 


\section{References}

1 Buckwalter, J. A. \& Mankin, H. J. Articular cartilage: tissue design and chondrocyte-matrix interactions. Instr Course Lect 47, 477-486 (1998).

2 Hasler, E. M., Herzog, W., Wu, J. Z., Muller, W. \& Wyss, U. Articular cartilage biomechanics: theoretical models, material properties, and biosynthetic response. Crit Rev Biomed Eng 27, 415-488 (1999).

3 Lin, Z., Willers, C., Xu, J. \& Zheng, M. H. The chondrocyte: biology and clinical application. Tissue Eng 12, 1971-1984, doi:10.1089/ten.2006.12.1971 (2006).

4 Poole, A. R. et al. Composition and structure of articular cartilage: a template for tissue repair. Clin Orthop Relat Res, S26-33 (2001).

5 Brittberg, M. et al. Treatment of deep cartilage defects in the knee with autologous chondrocyte transplantation. $N$ Engl $J$ Med 331, 889-895, doi:10.1056/NEJM199410063311401 (1994).

6 Harrison, P. E. et al. The in vitro growth of human chondrocytes. Cell Tissue Bank 1, 255-260 (2000).

7 Manning, W. K. \& Bonner, W. M., Jr. Isolation and culture of chondrocytes from human adult articular cartilage. Arthritis Rheum 10, 235-239 (1967).

8 Benya, P. D., Padilla, S. R. \& Nimni, M. E. Independent regulation of collagen types by chondrocytes during the loss of differentiated function in culture. Cell 15, 1313-1321, doi:0092-8674(78)90056-9 [pii] (1978).

9 Mayne, R., Vail, M. S., Mayne, P. M. \& Miller, E. J. Changes in type of collagen synthesized as clones of chick chondrocytes grow and eventually lose division capacity. Proc Natl Acad Sci U S A 73, 1674-1678 (1976).

10 von der Mark, K., Gauss, V., von der Mark, H. \& Muller, P. Relationship between cell shape and type of collagen synthesised as chondrocytes lose their cartilage phenotype in culture. Nature 267, 531-532 (1977).

11 Kang, S. W., Yoo, S. P. \& Kim, B. S. Effect of chondrocyte passage number on histological aspects of tissue-engineered cartilage. Biomed Mater Eng 17, 269276 (2007).

12 Schulze-Tanzil, G. et al. Redifferentiation of dedifferentiated human chondrocytes in high-density cultures. Cell Tissue Res 308, 371-379, doi:10.1007/ s00441-002-0562-7 (2002).

13 Chua, K. H., Aminuddin, B. S., Fuzina, N. H. \& Ruszymah, B. H. Insulintransferrin-selenium prevent human chondrocyte dedifferentiation and promote the formation of high quality tissue engineered human hyaline cartilage. Eur Cell Mater 9, 58-67; discussion 67 (2005).

14 Mandl, E. W. et al. Fibroblast growth factor-2 in serum-free medium is a potent mitogen and reduces dedifferentiation of human ear chondrocytes in monolayer culture. Matrix Biol 23, 231-241, doi:10.1016/j.matbio.2004.06.004 (2004).

15 Martin, I., Vunjak-Novakovic, G., Yang, J., Langer, R. \& Freed, L. E. Mammalian chondrocytes expanded in the presence of fibroblast growth factor 2 maintain the ability to differentiate and regenerate three-dimensional cartilaginous tissue. Exp Cell Res 253, 681-688, doi:10.1006/excr.1999.4708 (1999).

16 Stewart, M. C., Saunders, K. M., Burton-Wurster, N. \& Macleod, J. N. Phenotypic stability of articular chondrocytes in vitro: the effects of culture models, bone morphogenetic protein 2, and serum supplementation. J Bone Miner Res 15, 166-174, doi:10.1359/jbmr.2000.15.1.166 (2000). 
van Osch, G. J., Marijnissen, W. J., van der Veen, S. W. \& Verwoerd-Verhoef, H. L. The potency of culture-expanded nasal septum chondrocytes for tissue engineering of cartilage. Am J Rhinol 15, 187-192 (2001).

18 Kuettner, K. E. et al. Synthesis of cartilage matrix by mammalian chondrocytes in vitro. II. Maintenance of collagen and proteoglycan phenotype. J Cell Biol 93, 751-757 (1982).

19 Kuettner, K. E., Pauli, B. U., Gall, G., Memoli, V. A. \& Schenk, R. K. Synthesis of cartilage matrix by mammalian chondrocytes in vitro. I. Isolation, culture characteristics, and morphology. J Cell Biol 93, 743-750 (1982).

20 Mandl, E. W., van der Veen, S. W., Verhaar, J. A. \& van Osch, G. J. Multiplication of human chondrocytes with low seeding densities accelerates cell yield without losing redifferentiation capacity. Tissue Eng 10, 109-118, doi:10.1089/107632704322791754 (2004).

21 Watt, F. M. Effect of seeding density on stability of the differentiated phenotype of pig articular chondrocytes in culture. J Cell Sci 89 ( Pt 3), 373-378 (1988).

Murphy, C. L., Thoms, B. L., Vaghjiani, R. J. \& Lafont, J. E. Hypoxia. HIFmediated articular chondrocyte function: prospects for cartilage repair. Arthritis Res Ther 11, 213, doi:10.1186/ar2574 (2009).

23 van der Windt, A. E. et al. Physiological tonicity improves human chondrogenic marker expression through nuclear factor of activated T-cells 5 in vitro. Arthritis research \& therapy 12, R100, doi:10.1186/ar3031 (2010).

24 Elima, K. \& Vuorio, E. Expression of mRNAs for collagens and other matrix components in dedifferentiating and redifferentiating human chondrocytes in culture. FEBS Lett 258, 195-198 (1989).

25 Liu, H., Lee, Y. W. \& Dean, M. F. Re-expression of differentiated proteoglycan phenotype by dedifferentiated human chondrocytes during culture in alginate beads. Biochim Biophys Acta 1425, 505-515 (1998).

26 Tallheden, T. et al. Gene expression during redifferentiation of human articular chondrocytes. Osteoarthritis Cartilage 12, 525-535, doi:10.1016/ j.joca.2004.03.004 (2004).

27 Aulthouse, A. L. et al. Expression of the human chondrocyte phenotype in vitro. In Vitro Cell Dev Biol 25, 659-668 (1989).

28 Benya, P. D. \& Shaffer, J. D. Dedifferentiated chondrocytes reexpress the differentiated collagen phenotype when cultured in agarose gels. Cell 30, 215-224 (1982).

29 Buschmann, M. D., Gluzband, Y. A., Grodzinsky, A. J., Kimura, J. H. \& Hunziker, E. B. Chondrocytes in agarose culture synthesize a mechanically functional extracellular matrix. J Orthop Res 10, 745-758, doi:10.1002/ jor.1100100602 (1992).

30 Perka, C. et al. Joint cartilage repair with transplantation of embryonic chondrocytes embedded in collagen-fibrin matrices. Clin Exp Rheumatol 18, 1322 (2000).

31 Perka, C., Spitzer, R. S., Lindenhayn, K., Sittinger, M. \& Schultz, O. Matrixmixed culture: new methodology for chondrocyte culture and preparation of cartilage transplants. J Biomed Mater Res 49, 305-311 (2000).

32 Hauselmann, H. J. et al. Phenotypic stability of bovine articular chondrocytes after long-term culture in alginate beads. J Cell Sci 107 ( Pt 1), 17-27 (1994).

33 Hauselmann, H. J. et al. Adult human chondrocytes cultured in alginate form a matrix similar to native human articular cartilage. Am J Physiol 271, C742-752 (1996). 
34 Homicz, M. R. et al. Human septal chondrocyte redifferentiation in alginate, polyglycolic acid scaffold, and monolayer culture. Laryngoscope 113, 25-32, doi:10.1097/00005537-200301000-00005 (2003).

35 Domm, C., Schunke, M., Christesen, K. \& Kurz, B. Redifferentiation of dedifferentiated bovine articular chondrocytes in alginate culture under low oxygen tension. Osteoarthritis Cartilage 10, 13-22, doi:10.1053/joca.2001.0477 (2002).

36 Qiao, B., Padilla, S. R. \& Benya, P. D. Transforming growth factor (TGF)-betaactivated kinase 1 mimics and mediates TGF-beta-induced stimulation of type II collagen synthesis in chondrocytes independent of Col2al transcription and Smad3 signaling. J Biol Chem 280, 17562-17571, doi:10.1074/jbc.M500646200 (2005).

37 Schallmoser, K. et al. Rapid large-scale expansion of functional mesenchymal stem cells from unmanipulated bone marrow without animal serum. Tissue engineering 14, 185-196 (2008).

38 Welting, T. J. et al. Inhibition of cyclooxygenase-2 impacts chondrocyte hypertrophic differentiation during endochondral ossification. European cells \& materials 22, 420-436; discussion 436-427 (2011).

39 Farndale, R. W., Buttle, D. J. \& Barrett, A. J. Improved quantitation and discrimination of sulphated glycosaminoglycans by use of dimethylmethylene blue. Biochim Biophys Acta 883, 173-177 (1986).

40 Farndale, R. W., Sayers, C. A. \& Barrett, A. J. A direct spectrophotometric microassay for sulfated glycosaminoglycans in cartilage cultures. Connect Tissue $\operatorname{Res}$ 9, 247-248 (1982).

41 Lefebvre, V. \& Smits, P. Transcriptional control of chondrocyte fate and differentiation. Birth Defects Res C Embryo Today 75, 200-212, doi:10.1002/ bdrc.20048 (2005).

42 de Haart, M., Marijnissen, W. J., van Osch, G. J. \& Verhaar, J. A. Optimization of chondrocyte expansion in culture. Effect of TGF beta-2, bFGF and L-ascorbic acid on bovine articular chondrocytes. Acta Orthop Scand 70, 55-61 (1999).

43 Bernstein, P. et al. Pellet culture elicits superior chondrogenic redifferentiation than alginate-based systems. Biotechnol Prog 25, 1146-1152, doi:10.1002/ btpr.186 (2009).

$44 \mathrm{Fu}, \mathrm{N}$. et al. Comparison of protein and mRNA expression evolution in humans and chimpanzees. PLoS One 2, e216, doi:10.1371/journal.pone.0000216 (2007).

45 Haudenschild, D. R., Chen, J., Pang, N., Lotz, M. K. \& D'Lima, D. D. Rho kinase -dependent activation of SOX9 in chondrocytes. Arthritis and rheumatism 62, 191 -200, doi:10.1002/art.25051 (2010).

46 Sudbeck, P. \& Scherer, G. Two independent nuclear localization signals are present in the DNA-binding high-mobility group domains of SRY and SOX9. The Journal of biological chemistry 272, 27848-27852 (1997).

47 Binette, F. et al. Expression of a stable articular cartilage phenotype without evidence of hypertrophy by adult human articular chondrocytes in vitro. J Orthop Res 16, 207-216, doi:10.1002/jor.1100160208 (1998).

48 Hirao, M., Tamai, N., Tsumaki, N., Yoshikawa, H. \& Myoui, A. Oxygen tension regulates chondrocyte differentiation and function during endochondral ossification. J Biol Chem 281, 31079-31092, doi:M602296200 [pii] 10.1074/jbc.M602296200 (2006).

49 Morita, K. et al. Reactive oxygen species induce chondrocyte hypertrophy in endochondral ossification. $J$ Exp Med 204, 1613-1623, doi:jem.20062525 [pii] 10.1084/jem.20062525 (2007). 
50 Zuscik, M. J., Hilton, M. J., Zhang, X., Chen, D. \& O'Keefe, R. J. Regulation of chondrogenesis and chondrocyte differentiation by stress. J Clin Invest 118, 429438 (2008).

51 Yang, K. G. et al. Altered in vitro chondrogenic properties of chondrocytes harvested from unaffected cartilage in osteoarthritic joints. Osteoarthritis Cartilage 14, 561-570, doi:10.1016/j.joca.2005.12.002 (2006).

52 Barry, F., Boynton, R. E., Liu, B. \& Murphy, J. M. Chondrogenic differentiation of mesenchymal stem cells from bone marrow: differentiation-dependent gene expression of matrix components. Exp Cell Res 268, 189-200, doi:10.1006/ excr.2001.5278 (2001).

53 Cals, F. L., Hellingman, C. A., Koevoet, W., Baatenburg de Jong, R. J. \& van Osch, G. J. Effects of transforming growth factor-beta subtypes on in vitro cartilage production and mineralization of human bone marrow stromal-derived mesenchymal stem cells. J Tissue Eng Regen Med 6, 68-76, doi:10.1002/term.399 (2012).

54 Mankin, H. J., Dorfman, H., Lippiello, L. \& Zarins, A. Biochemical and metabolic abnormalities in articular cartilage from osteo-arthritic human hips. II. Correlation of morphology with biochemical and metabolic data. $J$ Bone Joint Surg Am 53, 523-537 (1971).

55 O'Driscoll, S. W. et al. Validation of a simple histological-histochemical cartilage scoring system. Tissue Eng 7, 313-320, doi:10.1089/10763270152044170 (2001).

56 Pritzker, K. P. et al. Osteoarthritis cartilage histopathology: grading and staging. Osteoarthritis Cartilage 14, 13-29, doi:10.1016/j.joca.2005.07.014 (2006). 



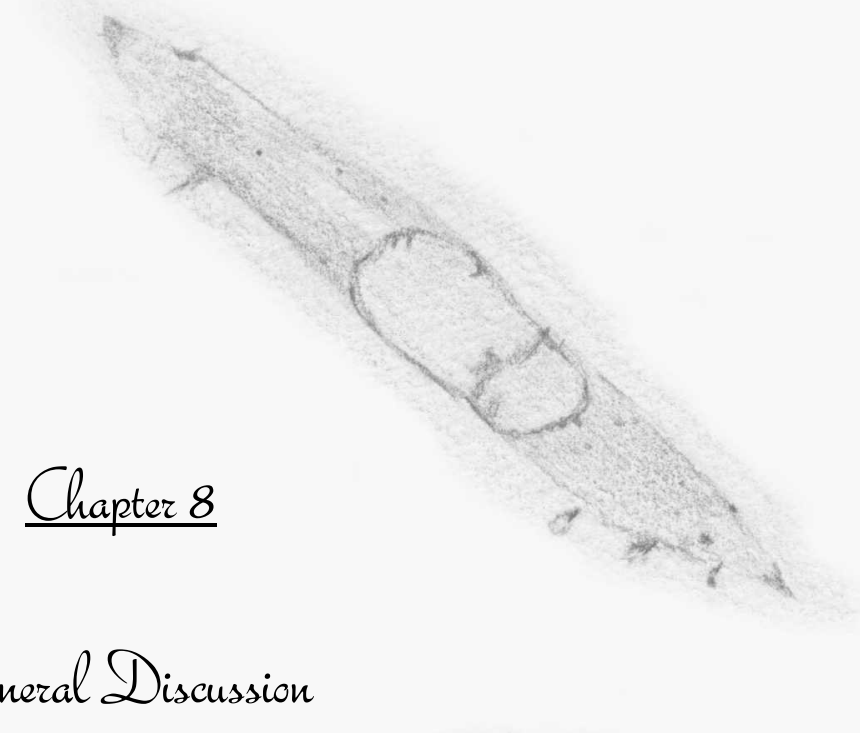

\section{General Discussion}

\section{Adapted from:}

Targeting Inflammatory Processes for Optimization of Cartilage Homeostasis and Repair Techniques

Marjolein M.J. Caron, Tim J.M. Welting, Lodewijk W, van Rhijn and Pieter J. Emans :

Department of Orthopaedic Surgery, Maastricht University Medical Center, Maastricht, the Netherlands In: ICRS: Developing Insights in Cartilage Repair

Edited by: Prof. L. Peterson and P.J. Emans, Springer-Verlag London (Accepted for publication)

\section{Endochondral Bone Formation as Blueprint for Regenerative Medicine}

Pieter J. Emans, Marjolein M.J. Caron, Lodewijk W. van Rhijn and Tim.J.M. Welting

Department of Orthopaedic Surgery, Maastricht University Medical Center, Maastricht, the Netherlands

In: Tissue Regeneration - From Basic Biology to Clinical Application (2012)

Edited by: Prof. J. Davies, ISBN: 978-953-51-0387-5, InTech 

Repair of articular cartilage is challenging due to the complex biomechanical function of cartilage and the biochemical composition of its extracellular matrix. Furthermore, its intrinsic self-repair capacity is limited due to lack of vascularization and limited source of progenitor cells. Understanding mechanisms of (progenitor) cell commitment into the chondrogenic lineage and prevention of chondrocyte hypertrophy are of importance to optimize cartilage repair strategies. Chondrogenic differentiation is a tightly regulated process in which many factors regulate the discrete stages of the differentiation program (Chapter 1). The work described in this thesis aimed to explore novel perspectives to increase the chondrogenic differentiation capacity of progenitor cells and modulate their chondrogenic outcome.

\section{Stimulating chondrogenic differentiation of progenitor cells}

Progenitor cells as a source for therapeutic strategies have various beneficial properties; such as their multipotency and ability to secrete paracrine factors to modulate their microenvironment and own cellular (differentiation) processes. This makes progenitor cells attractive candidates for cell-based cartilage regenerative approaches and it is crucial to better understand the regulatory mechanisms of their differentiation into cartilaginous tissues.

Several approaches were explored to enhance the chondrogenic differentiation capacity of

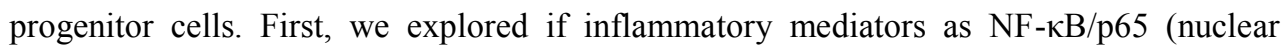
factor kappa-light-chain-enhancer of activated B-cells/subunit p65) and cyclooxygenase (COX)-2 have a role in the onset of chondrogenic differentiation, as they are both described to be essential for early bone fracture healing. The aforementioned inflammatory factors are able to induce expression of growth factors, and we determined which of a subset of BMPs (bone morphogenic proteins) would be most optimal for increasing the chondrogenic potential of progenitor cells. Furthermore, as an alternative approach to influence the magnitude of chondrogenic differentiation we studied if increasing culture medium osmolarity to mature articular cartilage physiological levels was beneficial for the chondrogenic outcome, and if the NF- $\mathrm{BB}$ family member Nfat5 (nuclear factor of activated $\mathrm{T}$-cells 5) is involved in this process. 


\subsection{Role for inflammatory mediators in early chondrogenic differentiation}

The general understanding on the role of inflammatory molecules and articular cartilage is a katabolic one. Inflammatory mediators (mainly originating from the synovium ${ }^{1}$ ) initiate and/or maintain cartilage breakdown processes seen in osteoarthritis (OA) and rheumatoid arthritis (RA), resulting in decreased cartilage functioning and chondrocyte viability ${ }^{2}$. Important inflammatory molecules in progression of $\mathrm{OA}$ are e.g. NF-KB/p65, tumor necrosis factor alpha (TNF $\alpha)$, interleukins, cyclooxygenases and prostaglandins ${ }^{2,3}$. Interestingly, despite the overall inflammatory environment in an OA joint, osteoarthritis often induces osteophyte formation. Basically, these are ossifying and isolated ectopic cartilaginous tissues near the synovial membrane, which are committed to follow the process of endochondral ossification ${ }^{4,5}$. Moreover, endochondral bone fracture healing depends on an inflammatory haematoma formation ${ }^{6-8}$, where early chondrogenic differentiation also takes place in an inflammatory environment. These are two examples where initiation of chondrogenic differentiation is permitted in an inflammatory environment; we therefore hypothesized that inflammatory mediators are a valuable tool to influence in vitro chondrogenic differentiation of progenitor cells. In Chapter 2 we showed that indeed chondrogenic differentiation of chondroprogenitor cells is, at least in part, determined by early activation of $\mathrm{NF}-\kappa \mathrm{B} / \mathrm{p} 65^{9}$. These results are supported by several other studies showing that factors secreted by OA chondrocytes are able to induce chondrogenic differentiation of mesenchymal progenitor cells ${ }^{10,11}$ Chondrogenic differentiation in vivo during skeletal development is not yet reported to be associated with inflammatory factors. We found expression of COX-2, inducible nitric oxide synthase (iNOS) and p65 in the resting zone of the growth plate. However it remains elusive at this time if and how these factors are in vivo stimulated by inflammatory signaling molecules as TNF $\alpha$ or interleukins and contribute to growth plate development. Inflammatory signaling in this context could arise from paracrine signaling from neighboring chondrocytes or even bone marrow. Alternatively, growth factors might induce the expression of these inflammatory mediators; it is known that transforming growth factor (TGF)- $\beta$-receptor (TGFR), insulinlike growth factor (IGF)-receptor (IGFR) and BMP signaling activate NF- $\kappa \mathrm{B}$ and expression of chondrogenic markers ${ }^{12-14}$. In addition, an important regulator of chondrogenic progenitor cells; Bapx1/Nkx3.2 (Bagpipe homeobox homolog 1/ NK3 homeobox 2), is also described to activate the NF- $\mathrm{B}$ pathway and subsequent chondrocyte viability ${ }^{15}$. Furthermore, this Bapx1/Nkx3.2 activity can be maintained by BMP signals ${ }^{16}$.

Progenitor cells produce several growth factors (BMP-2, BMP-4, TGF- $\beta 1$, IGF-1, fibro- 
blast growth factor (FGF)-2, FGF-3, vascular endothelial growth factor (VEGF), hepatocyte growth factor (HGF), and others) after stimulation with lipopolysaccharide (LPS) or $\mathrm{TNF} \alpha$ in a NF- $\kappa \mathrm{B}$ dependent manner which might initiate/maintain the chondrogenic differentiation process ${ }^{9,17}$. We have also shown that activated $p 65$ was found to be a crucial factor in driving an early transient induction of Sox9 (SRY (sex determining region Y) box 9), a key event in early chondrogenic differentiation. This early transient Sox9 induction at the start of chondrogenic differentiation is accompanied by a similar early transient expression of COX-2, iNOS, interleukin 6 (Il-6) and TNF $\alpha$. Although these factors are described to be involved in endochondral ossification processes ${ }^{18,19}$, their function in early chondrogenic differentiation is currently unknown. Gain- and loss-of-function experiments are needed to acquire more insight in their early chondrogenic function (The influence of COX-2 on chondrogenic differentiation is discussed in paragraph 2.2).

Further research is needed to unravel how inflammatory processes influence important key chondrogenic pathways and how this can be deployed for cartilage regenerative medicine. As TNF $\alpha$ or LPS might be counterintuitive (non-physiological) inducers of inflammatory $(\mathrm{NF}-\mathrm{\kappa B})$ signaling for progenitor-based cartilage strategies, using alternative approaches as dedicated biological (growth factors)/pharmacological agents targeting inflammatory pathways are expected to provide the right tools for cartilage regenerative medicine approaches.

We determined (Chapter 4) that both BMP-2 and BMP-7 in equimolar concentrations were able to enhance chondrogenic differentiation from progenitor cells, with a more pronounced effect for BMP-7. Moreover, BMP-2 is able to increase the early Sox9 expression levels in a NF- $\mathrm{BB} / \mathrm{p} 65$ dependent way ${ }^{9}$ (Chapter 1). It would therefore be tempting to determine if BMP-7 (Chapter 4) is also able to execute this effect via increased $\mathrm{NF}-\mathrm{\kappa B} / \mathrm{p} 65$ signaling (maybe even more than BMP-2), which results in the increased early Sox 9 expression and ensuing collagen type II (Col2a1)expression. Interestingly, BMP-7 (but not BMP-2) was able to prevent induction of chondrocyte hypertrophy. This will be discussed in paragraph 2.1 .

Based on the above and previous literature, growth factors and inflammatory mediators are in a tight interplay in regulating early chondrogenic differentiation. The use of anti-inflammatory agents might therefore contribute to poor self-repair capacity of the cartilage as the few progenitor cells residing in the tissue ${ }^{20}$ are now prevented from the potential to repair the damaged cartilage. This potentially poses a stalemate in the use of anti-inflammatory agents for the treatment of OA. 


\subsection{Changing the micro-environment of progenitor cells to the physiological environment}

\section{of cartilage}

The extracellular matrix (ECM) of articular cartilage is normally hypoxic due to the absence of blood vessels ${ }^{21}$. It is generally known that hypoxia is essential for chondrocyte survival in vivo; it inhibits chondrocyte proliferation in the growth plate allowing increased extracellular matrix accumulation ${ }^{22}$. Consequently, mature chondrocyte cultures (passaging and redifferentiation) benefit from culturing under low oxygen levels. In addition, several studies also showed increased chondrogenic potential from progenitor cells when cultured under hypoxia ${ }^{17,22-24}$. Specifically, hypoxia-induced Hif-1 $\alpha$ (hypoxia-inducible factor 1, alpha subunit) expression is able to transcriptionally activate Sox9 expression in prechondrogenic cells ${ }^{23}$. Furthermore, hypoxic conditions also increase growth factor expression in human mesenchymal stem cells via a NF- $\kappa \mathrm{B}$ dependent manner ${ }^{17}$. It would be tempting to determine whether and how hypoxia or Hif- $1 \alpha$ are involved in the above described induction of early chondrogenic differentiation by inflammatory mediators. The above suggests that chondrogenic differentiation from progenitor cells can benefit from natural environmental characteristics of the cartilage ECM. Another important hallmark of the cartilage ECM is increased osmolarity (ranging from $350-480 \mathrm{mOsm}$ ) as compared to plasma levels $(\sim 280 \mathrm{mOsm})^{25,26}$. Sulphated glycosaminoglycan (GAG) chains on the proteoglycans are responsible for a high fixed negative charge density, which attracts mobile cations and water from the ECM-environment ${ }^{27,28}$. Together with the quality of the collagen network, this determines the osmolarity of the cartilage extracellular fluid and provides compressive strength and flexibility to the cartilage tissue. Several studies have shown that culturing mature human articular chondrocytes in vitro under chondrocyte physiological osmolarity results in increased ECM synthesis ${ }^{26,29-31}$. In Chapter 3 we describe that also chondrocyte progenitor cells are osmolarity-responsive and their chondrogenic potential is increased when cultured in medium with increased osmolarity (+100 mOsm). As we used sodium chloride to increase the medium osmolarity, the effects on chondrogenic differentiation might also arise from sodium of chloride ion specific effects. However, in a previous study ${ }^{30}$ using human articular chondrocytes, we used sucrose or N-methyl-D-glucamine (NMDG-CL; a bulky substitute for small cations that are impermeable to almost all known channels) to adjust the medium to $+100 \mathrm{mOsm}$ and found similar results as with sodium chloride. This indicates that the effects of increased osmolarity on chondrogenic outcome were mainly caused by hypertonic stress rather than sodium of chloride ion specific effects. As increasing the osmolarity of a cellular environ- 
ment in vitro or in vivo is relatively simple and safe, this holds a promise for future optimization of progenitor cell-based cartilage repair approaches. Increasing the osmolarity on sites where in vivo progenitor cells reside (periosteum, synovium, superficial layer of cartilage) might induce or support chondrogenic differentiation which can repair damaged cartilage. Combining cells with biomaterials (gells) which have an increased osmolarity is a tempting possibility. During cartilage degeneration (OA), GAG content is decreased and as a result also the osmolarity of the cartilage $\mathrm{ECM}^{32,33}$. Increasing osmolartiy in these conditions back to normal physiological conditions might stimulate cartilage repair mechanisms in the OA joint.

Nfat5 is an important regulator in the cellular reaction to hypertonic stress, and indeed, our results showed that increased osmolarity correlates with increased Nfat5 expression and knockdown of Nfat5 under increased osmolarity $(+100 \mathrm{mOsm})$ conditions resulted in downregulation of chondrogenic markers back to control levels. Overexpression of Nfat5 will reveal whether Nfat5 is enough to increase chondrogenic differentiation from progenitor cells independent of increased osmolarity. In addition, we found an osmolarityindependent function of Nfat5 during chondrogenic differentiation, as Nfat5 is already expressed during in vitro chondrogenic differentiation and knockdown under control plasma osmolarity levels $(\sim 280 \mathrm{mOsm})$ resulted in reduced expression of Col2a1, aggrecan (Acan), Sox9, collagen type X (Col10a1) and runt-related transcription factor 2 (Runx2). This is in line with a growing body of evidence indicating that Nfat5 has diverse osmolarity-independent functions ${ }^{34-36}$. Further analysis will reveal how Nfat5 influences chondrogenic differentiation from progenitor cells. One of the possibilities we explored is a transcriptional interaction between Nfat5 and the early expression of Sox9. ChIP (chromatin immuno-precipitation) experiments would be able to elucidate a direct transcriptional interaction between these two.

\subsection{Importance of activation of Sox 9 during early and late chondrogenic differentiation}

We identified two potential activators of the first Sox9 expression peak during early chondrogenic differentiation; both $\mathrm{NF}-\kappa \mathrm{B} / \mathrm{p} 65$ and $\mathrm{Nfat5}$ were able to regulate its expression. However, we know little of the early transient Sox9 function; how it is regulated and what it regulates. It became clear is that specific interference with early Sox 9 function abrogates the complete downstream chondrogenic differentiation program. This indicates that very early in differentiation crucial decisions are being made that impact the result of the differentiation process. Instant hypertrophic differentiation (data not shown) is 
one of the possible explanations for the chondrogenic failure upon early Sox 9 interference, as well as Sox9-dependent epigenetic priming of chondrogenic loci. The second, late Sox9 peak co-incidences with ECM synthesis and is thus expected to transcriptionally serve ECM synthesis. We expect that early Sox 9 expression is transcriptionally regulated via different mechanisms as compared to late Sox9 induction. Transcription of Sox9 has been reported to involve binding of general transcriptional enhancers and factors including CCAAT-binding factor, CREB (cAMP response element-binding), NF-kB/p65, Hif-1 $\alpha$ and $\operatorname{Sp} 1$ (specificity protein 1$)^{23,37,38}$ to the proximal promoter. In this light it would therefore be intriguing to determine whether these Sox 9 transcriptional activators act in driving early Sox9 expression, or are specific to late Sox9 expression. A yeast one-hybrid screening approach would be able to identify novel transcription factors for Sox 9 in an unbiased way, providing the opportunity to dissect mechanisms that specifically regulate early versus late Sox 9 expression. On the other hand, this bi-phasic Sox 9 expression might also indicate differential regulation of target genes. Sox9 is described to be essential for transcription of important chondrogenic genes as Col2a1, Acan, collagen type IX (Co19a1), collagen type XXVII (Col27a1) and matrilin 1 (Matn1) ${ }^{39-47}$, however these reports did not discriminate if these involved transcription by early or late Sox 9 activation. Gain- and lossof-function studies concerning Sox9 early and late in differentiation will contribute to the understanding of the role of both Sox 9 expression peaks.

Taken together, novel findings have been made on understanding the role of Sox 9 in (early) chondrogenic differentiation, however, in general, little knowledge is transferred to the cartilage regenerative medicine field. As Sox9 is a key transcription factor for chondrogenic lineage commitment and maintenance, activation of Sox9 is essential for progenitor cell-based cartilage regenerative techniques. Stimulating Sox9 activation by inflammatory mediators, increased osmolarity, hypoxia or other approaches are promising tools for augmenting progenitor cell-based cartilage regeneration.

\section{Modulating cartilage hypertrophic differentiation}

Equally important for obtaining an optimal chondrogenic phenotype is not only to stimulate progenitor cells towards chondrocytes but also prevent them from hypertrophy. Unfortunately, when stimulating progenitor cells towards the chondrogenic lineage they 
almost inevitable become hypertrophic, as they express typical hypertrophic molecules as Runx2, Col10a, alkaline phosphatase (Alp) and myocyte-specific enhancer 2c (Mef2c). This was also observed in our studies where progenitor cells were stimulated with inflammatory mediators (Chapter 2), increased osmolarity conditions (Chapter 3) and BMP-2 supplementation (Chapter 4). These factors not only induced chondrogenic genes as Sox9, Col2a1 and Acan but this was followed by an increase in expression of the above mentioned hypertrophic genes. As chondrogenic differentiation from progenitor cells is an essential part of the endochondral ossification pathway, it might not be surprising that hypertrophy occurs. However, for cartilage regenerative approaches this is of course not desirable and needs to be addressed with great care.

Although it is a possibility that the cartilage micro-environment (increased osmolarity, hypoxia, ECM structure, parathyroid hormone related peptide (PTHrP)) guides (differentiating) progenitor cells towards functional cartilage and prevents the unwanted hypertrophy, it could still be advantageous for ex vivo induced cartilage regenerative medicine approaches to combine this with an anti-hypertrophic strategy. We found several novel leads in suppressing the chondrocyte hypertrophy during chondrogenic differentiation of progenitor cells. Firstly, to determine hypertrophy suppressing effects one needs a chondrogenic culture model that becomes hypertrophic by nature. Both our frequently used in vitro cell models; ATDC5 and monolayer-differentiated human bone marrow stem cells (hBMSCs) do so. Chapter 4 and 5 show that the hypertrophic phenotype can be enhanced after BMP-2 stimulation. Interestingly, we found that BMP-7 was able to suppress the chondrocyte hypertrophy. In addition, we identified a role for COX-2 in hypertrophy as inhibition of COX-2 resulted in suppressed hypertrophy while leaving chondrogenic differentiation unaltered ${ }^{48}$. Moreover, we determined how this might be explained by differential prostaglandin synthesis under COX-2 and COX-1 specific inhibition.

Chondrocyte hypertrophy is not only an unwanted phenomenon in chondrogenic differentiation from progenitor cells but is also a hallmark of early OA in mature mature chondrocytes and hampers the outcome of chondrocyte-based cartilage repair approaches. A cell culture system displaying a chondrocyte hypertrophic phenotype can be of importance when studying this process on a molecular basis. To determine differences in chondrogenic and hypertrophic marker expression we compared different culture models for human articular chondrocytes (HACs) ${ }^{49}$. Potential chondrogenic-stimulating and hypertrophic-suppressing agents identified in our previous studies could also be applied for 
mature articular chondrocytes.

\subsection{Differential effects of BMP-2 and BMP-7 on chondrogenic outcome}

As described above both BMP-2 and BMP-7 stimulation during chondrogenic differentiation of progenitor cells resulted in an increased chondrogenic marker expression. Runx2 was differentially influenced by the BMPs; increased after BMP-2 stimulation and suppressed after BMP-7 stimulation, which might explain the suppressed hypertrophy in these cultures. Studies into the possible underlying mechanism revealed the Runx2 transcriptional repressor Bapx1/Nkx3.2 as a potential candidate ${ }^{50-53}$. Bapx1/Nkx3.2 is an important mediator in early (somitic) chondrogenic differentiation as it directly inhibits the expression of Runx 2 and increases the expression of Sox9 allowing chondrogenic induction $^{16,54}$. Sox 9 in turn increases the expression of Bapx1/Nkx3.2 establishing a positive feedbackloop for chondrogenic differentiation and maintenance. During the course of chondrogenic differentiation the expression of Bapx1/Nkx3.2 is lost and expression of Runx2 increases again, tipping the balance towards chondrocyte hypertrophy ${ }^{50,51,55}$. In support of this hypothesis we found that the decreased hypertrophy in the BMP-7 condition could be attributed to increased Bapx1/Nkx3.2 expression in these cells, and that the hypertrophy-suppressing action of BMP-7 was reversed after knock-down of Bapx1/ Nkx3.2. As BMP signals play an essential role in induction of Sox9 induced early chondrogenic differentiation, it is unknown if BMP-2 and BMP-7 are able to induce this effect on the same magnitude. Our preliminary data show that also the early Sox9 expression levels can be enhanced by both BMPs, with a more pronounced effect evoked by BMP-7 (Figure 8.1). In addition, Bapx1/Nkx3.2 also activates NF- $\mathrm{kB} / \mathrm{p} 65$ signalling ${ }^{15,56}$, and it would be interesting to determine if BMP-7 is able to signal through the NF- $\mathrm{BB} / \mathrm{p} 65$ pathway during early chondrogenic differentiation. This might be an explanation on how BMP-7 is able to induce or sustain Bapx1/Nkx3.2 signalling which results in suppressed hypertrophy at later stages in chondrogenic differentiation. Why BMP-2 does not result in similar increase in Bapx1/Nkx3.2 expression as BMP-7 is currently unknown. BMP-2 is described in literature to induce Bapx1/Nkx3.2-mediated Sox9 expression early in (somitic) chondrogenic differentiation ${ }^{16,54}$, and also induces early Sox 9 expression in our experiments however, it does not cause increased Bapx1/Nkx3.2 levels at later time points in differentiation. This might be a concentration-dependent effect, as BMP-7 in the same concentration is more potent in inducing Sox 9 expression and perhaps a higher concentration of BMP-2 is needed to obtain similar results. BMP-2 
might also signal at other time points in differentiation via alternative molecular pathways resulting in chondrocyte hypertrophy. BMP-2 and BMP-7 are described to differentially bind to the BMP receptors; BMP-2 preferentially binds to BMP receptor type IA (BMPRIA; Activin receptor like kinase (ALK) 3) and BMPR-IB (ALK6), whereas BMP-7 binds with higher affinity to ALK2 and BMPR-IB ${ }^{57-60}$ which could result in differential downstream signalling. In addition, specific Smad-signaling has been linked to the chondrogenic fate (Smad1/5/8 with chondrocyte hypertrophy and Smad2/3 with chondrogenic maintenance ${ }^{61}$ ), it is interesting to determine whether the differential action of BMP-2 and BMP-7 can be explained by specific Smad activation, potentially even at different time points in differentiation. More research is needed to define the role of both BMP-2 and BMP-7 during different phases of chondrogenic differentiation. Importantly, BMP-7 as opposed to BMP-2 appears to be a better candidate for optimization for cartilage regenerative strategies.

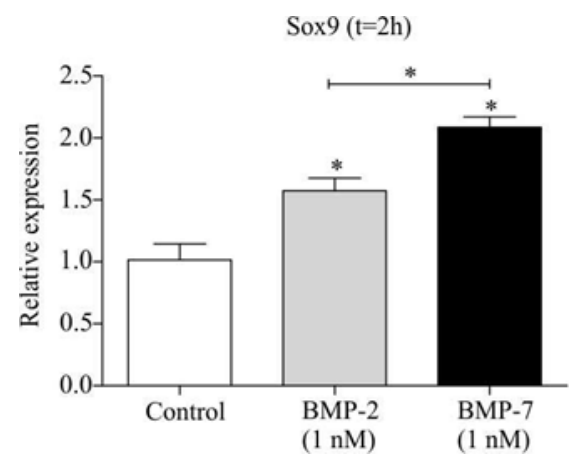

Figure 8.1: Differential effects of BMP-2 and BMP-7 on the chondrogenic outcome of ATDC5 cells

Equimolar concentrations ( $1 \mathrm{nM}$ ) of BMP-2 or BMP-7 were added from the start of chondrogenic differentiation of ATDC5. Sox9 mRNA expression was determined at 2 hours in differntiation. White bars represent control condition (set at 1.0), grey bars the BMP-2 condition and black bars the BMP-7 condition. In graphs, error bars represent mean \pm s.e.m. * indicates $p<0.05$ and is determined with respect to the control condition and between BMP-2 and BMP-7 conditions.

\subsection{A role for cell stress and COX-enzymes in chondrocyte hypertrophy}

For the articular cartilage component, osteoarthritis is in many ways similar to endochondral ossification, as in OA articular chondrocytes start to differentiate into hyper- 
trophic chondrocytes for reasons that are not yet completely understood ${ }^{62-65}$. Notably, the OA-associated inflammatory factors and accompanying cell stress are known to be involved in chondrocyte hypertrophic differentiation in the growth plate and may explain why articular cartilage is terminally differentiating in $\mathrm{OA}^{65,66}$. Stress-related pathways that are activated in growth plate chondrocyte hypertrophic differentiation involve ER (endoplasmic reticulum)-stress/unfolded protein response, oxidative stress ${ }^{67-69}$, advanced glycation end product-formation (AGEs) ${ }^{70-74}$, DNA damage and others ${ }^{66}$. In the growth plate these pathways are probably activated due to the rapid cell proliferation in the proliferative zone, reoxygenation of hypertrophic chondrocytes from the subchondral bone marrow, vast extra cellular matrix protein synthesis, etc. ${ }^{66}$. Moreover, correlating with hypertrophic differentiation, (pre-)hypertrophic cells also start to (re)express inflammatory molecules (COX-1, COX-2 ${ }^{48}, \mathrm{iNOS}^{75-77}, \mathrm{p} 65^{13,78,79}$ and others (our unpublished data), which we think enhance the intrinsic cellular capacity for hypertrophic differentiation. The message that should be taken from these observations is that failure of cartilage regenerative techniques due to formation of interlesional osteophytes, hypertrophic differentiation and calcification of cartilage grafts, may originate from similar processes. The pathways and processes stated above are therefore expected to be promising targets for avoiding failure due to terminal differentiation of the cartilage graft in the clinic.

Other authors have identified anti-oxidative components that decrease inflammatory signaling or chondrocyte hypertrophic differentiation. These components include $\mathrm{N}$-acetyl cysteine (NAC) ${ }^{80-82}$, resveratrol ${ }^{83-85}$, and even mechanical loading is described to lower the concentration of inflammatory mediators secreted during $\mathrm{OA}^{66,86}$. Similarly, parathyroid hormone related peptide (PTHrP) is known for its capacity to keep proliferating articular chondrocytes in their chondrocyte state and prevent them for further developing into hypertrophic chondrocytes ${ }^{87,88}$. Chapter 5 shows that pharmacological inhibition of the inflammatory enzyme cyclooxygenase-2 by e.g. NS398 and/or Celecoxib decreases the level of chondrocyte hypertrophic differentiation, even in BMP-2 induced chondrocyte hypertrophy ${ }^{48}$. This may provide a potential pharmacological approach to prevent or decrease chondrocyte hypertrophic differentiation in cartilage repair techniques. Indeed, preliminary data also show a delayed bone fracture healing and delayed calcification of the tissue generated by the in vivo bioreactor (IVB) when animals were treated with Celecoxib (Figure 8.2). In addition, our data show that systemic inhibition of COX-2 results in aberrant growth plate development due to a smaller hypertrophic zone ${ }^{48}$.

Remarkably, for COX-2 knockout (KO) mice a skeletal phenotype has not been described 
A

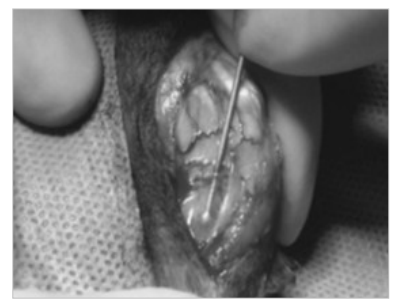

$D$

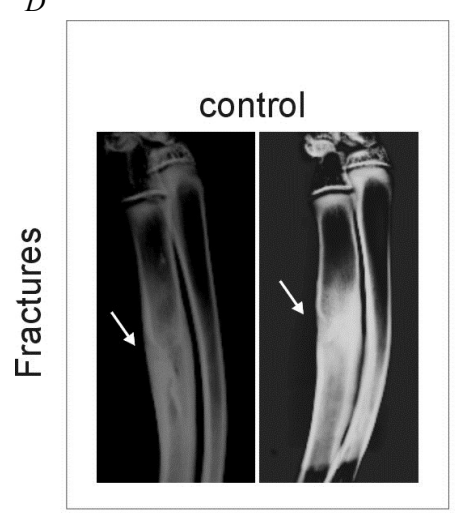

$E$

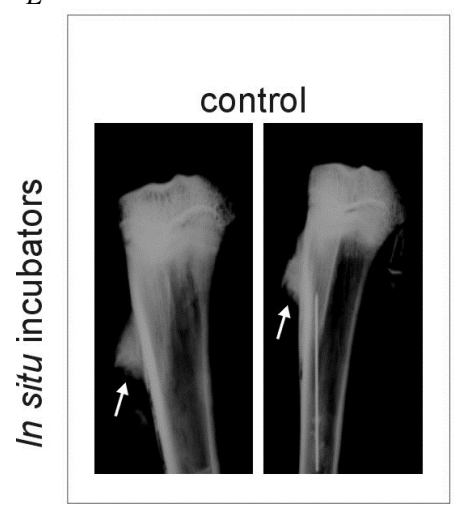

B
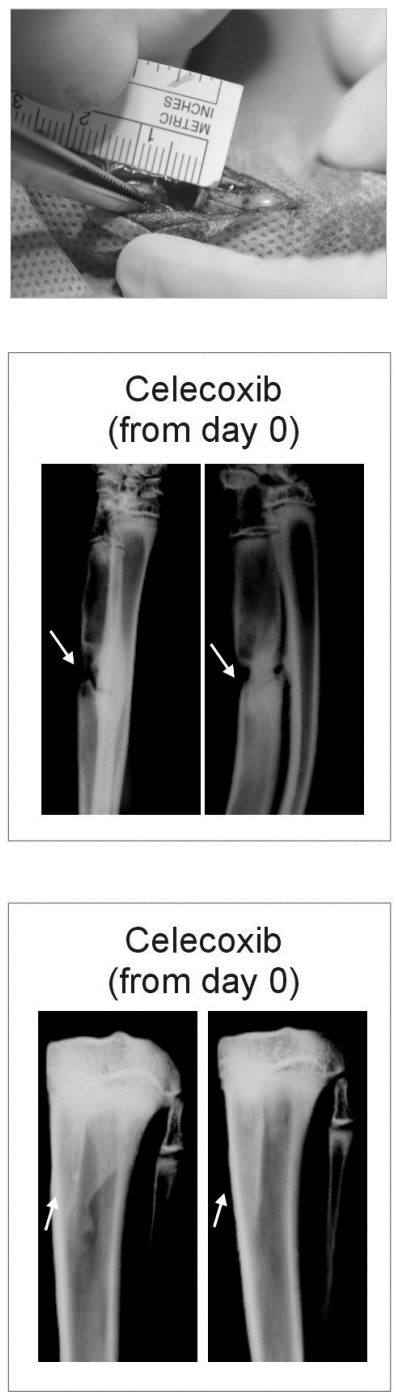

C
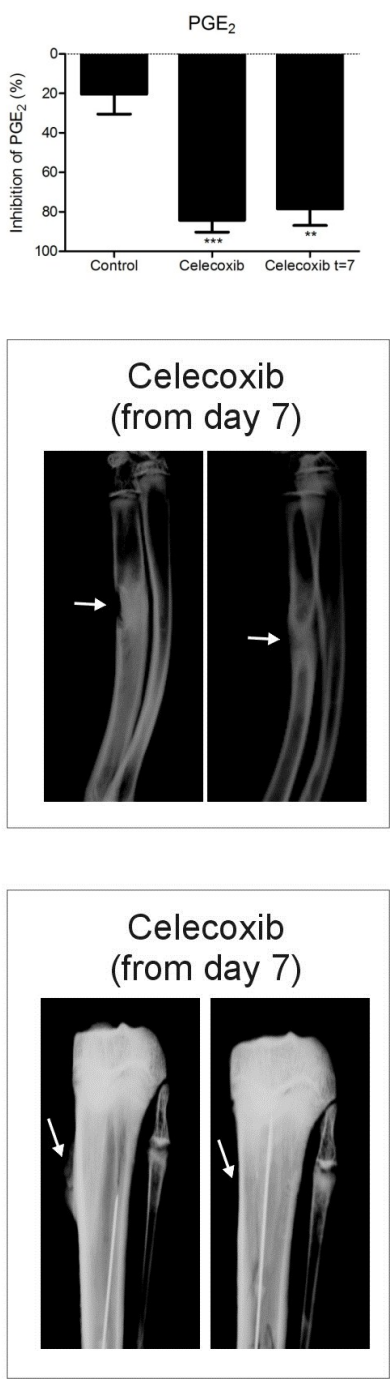

Figure 8.2: Different endochondral read-outs after systemic celecoxib treatment.

In a study using new-zealand white (NZW) rabbits $(1.8 \mathrm{~kg})$ celecoxib $(10 \mathrm{mg} / \mathrm{kg} /$ day $)$ was orally admitted for 21 days and the study consisted of three groups of six animals each (1 control group, 1 group with Celecoxib from day 0,1 group with Celecoxib from day 7 on. Endochondral study points per animal were non-critical ulnafracture of $0.5 \mathrm{~cm}$ and two in vivo bioreactors (IVB's) on tibial bones. $A$. The IVB: the periosteum is detached from the underlying bone using a tailor made periosteal elevator. The resulting subperiosteal space is filled with an agarose gel and after 3 weeks a cartilage callus is formed ${ }^{90} . B$. Induction of bone fracture: from the right ulna $0.5 \mathrm{~cm}$ bone was removed and the defect was measured to confirm the size of the defect. $C$. $\mathrm{PGE}_{2}$ plasma levels. Serum from day 21-animals was measured for $\mathrm{PGE}_{2}$ to confirm Celecoxib efficacy. $D$. Representative examples of X-rays of fracture healing of the three groups. The arrows indicate the site of the original fracture. E. Same for the IVB's. Calcified IVB's are visible on X-ray images. The arrows indicate the site where the IVB was created. 
but they do have problems with bone fracture healing ${ }^{89}$. It would be intriguing to re-evaluate if the COX-2 $\mathrm{KO}$ mice truly has no abnormalities in skeletal development and if so, how and why this differs from post-natal skeletal repair mechanisms. In addition, as COX-2 KO mice have no COX-2 protein and COX-2 inhibitors are not able to completely block total COX-2 function, a potential explanation for the discrepancies between the KO mice and COX-2-inhibitor treated animals might also be derived from here. Alternatively, there might also be differential rescue effects from COX-1 activity or differential prostaglandin synthesis between both situations

In vitro, COX-2 also shows two peak-expression patterns; one early in differentiation and one from day 7 in differentiation onwards. Until so far, we have inhibited COX-2 activity during the complete course of differentiation and future loss- and gain-of-function experiments at different time points and time spans in differentiation will reveal if these two COX-2 peaks have a different function in regulating chondrogenic differentiation from progenitor cells. It is thus important to realise that the same mediators might have differential effects on different phases during chondrogenic differentiation of progenitor cells via potential signalling through different molecular pathways. In addition to Celecoxib, other non-steroidal anti-inflammatory drugs (NSAIDs) are also described to influence the endochondral ossification process. Systemic NSAID treatment delays spinal fusion in patients and animal models ${ }^{91}$ and fracture healing of the long bones ${ }^{92-95}$. This property of NSAIDs is, however, exploited in the clinic to prevent periprosthetic heterotopic calcification after hip surgery ${ }^{96}$. However, as different NSAIDs have a different specificity on COX-enzyme inhibition it is possible that the other COX enzyme, COX-1, is also involved in mediating these effects. Indeed, as described in Chapter $6 \mathrm{COX}$ -1 specific inhibition resulted in decreased chondrogenic differentiation in vitro and our results also point towards a different regulation of the COX-enzymes in prostaglandin synthesis during different phases of chondrogenic differentiation. To use NSAIDs for successful progenitor cell-based cartilage regenerative medicine approaches it is necessary to determine if the COX-enzymes differentially regulate different phases of chondrogenic differentiation and if this is executed via $\mathrm{COX}$-specific prostaglandin production.

For each prostaglandin (PG) subtype several PG receptors have been reported ${ }^{97}$; EP1-4 are four receptor subtypes that can bind $\mathrm{PGE}_{2}$, DP1 can bind $\mathrm{PGD}_{2}$, and receptors of the I-type (IP) and F-type (FP) bind $\mathrm{PGI}_{2}$ and $\mathrm{PGF}_{2 \alpha}$ respectively. All of these receptors signal via G-proteins; however the intracellular signalling consequences differ ${ }^{97}$. EP(4) and IP signal via $G_{s}$-mediated increases in cAMP, and FP signals via $G_{q}$-mediated increases in 
intracellular calcium ${ }^{97}$, which might explain the differential effects of the different PGs on chondrogenic outcome. Elucidating how the specific prostaglandins influence regulatory molecular mechanisms in chondrogenic differentiation via potential differential receptor activation will provide more insight in how the prostaglandins affect the differentiation process and makes them a target for optimizing cartilage regeneration approaches as well.

\subsection{Culture methods for chondrocytes}

Progenitor cells are not the only source of cells which can be used for cartilage regenerative medicine, mature chondrocytes are another important cell-target. In Chapter 7 we compared different $2 \mathrm{D}$ and $3 \mathrm{D}$ culture methods for redifferentiation of $\mathrm{HACs}^{49}$. Both three-dimensional cultures were found to exhibit the highest chondrogenic capacity, suited for studies in chondrocyte-biology/maintenance. However, monolayer redifferentiation appears to result in expression of hypertrophic and mineralization markers and as such this model might be used for complementary studies addressing hypertrophic differentiation of chondrocytes. This study contributes to the understanding how these culture methods differentially determine the outcome of in vitro chondrogenic redifferentiation and may provide a rational behind the choice for one or the other culture system when addressing different research questions. For studying chondrocyte biology primary chondrocyte cultures in various 3D models are currently the primary choice and cell lines as SW1353 ${ }^{98}$, $\mathrm{T} / \mathrm{C} 28 \mathrm{a} 2 / 4^{99}$ or $\mathrm{C} 28 / \mathrm{I} 2^{100}$ are also described as reliable in vitro cell systems to investigate chondrocyte homeostasis. For studying the whole chondrogenic differentiation process from progenitor cells the ATDC5 ${ }^{101}, \mathrm{C} 3 \mathrm{H} 10 \mathrm{~T} 1 / 2^{102,103}$, or $\mathrm{USAC}^{104,105}$ cell lines are available, or primary mesenchymal stem cells can be used which can be isolated from various tissues. However, for studying chondrocyte hypertrophy not many standardized cell culture models are available. MCT cells ${ }^{106}$ are an example of a cell line which becomes hypertrophic within one day and ATDC5 cells can also be induced to become hypertrophic over long culturing period ${ }^{107}$. Using monolayer culture with primary (OA or healthy) chondrocytes might thus be an interesting option for studies into chondrocyte hypertrophy.

Importantly, a major discrepancy was found between mRNA and protein expression of COL2A1 and SOX9 in monolayer culture. Whereas COL2A1 and SOX9 mRNA expression is not or hardly induced in monolayer, COL2A1 and SOX9 proteins are expressed in monolayer culture. Notably, the magnitude of COL2A1 and SOX9 protein induction in monolayer was not significantly different from alginate or pellet culture. 
Including (quantitative) protein expression analysis is thus of importance when studying chondrocyte (re)differentiation and relying on frequently used gene expression analysis or GAG content alone is not sufficient and may lead to misinterpretation of the experimental data.

\section{Conclusions}

If progenitor cells are used for cartilage regenerative medicine approaches it is essential to realize that these cells differentiate via the endochondral ossification pathway towards chondrocytes and cartilage and that hypertrophy may be inevitable.

Manipulating the endochondral ossification processes can be used for optimizing cartilage repair techniques. The work described in this thesis aimed to find novel perspectives to increase the chondrogenic differentiation capacity from progenitor cells and modulate the chondrogenic outcome. Especially for progenitor cell based repair technologies, our novel insights could be employed to increase the differentiation potential of progenitor cells toward engineered cartilaginous tissue in vitro and in vivo.

An overview of the chondrogenic regulators which are studied in this thesis and their interactions in the context of chondrogenic differentiation from progenitor cells is depicted in Figure 8.3. Several approaches were explored to enhance the chondrogenic differentiation capacity of progenitor cells. First we determined that inflammatory mediators as $\mathrm{NF}-\kappa \mathrm{B} / \mathrm{p} 65$ have a role in the onset of chondrogenic differentiation, by increasesing expression of Sox 9 and important chondrogenic growth factors. We found that the use of a defined subset of growth factors, BMP-2 and BMP-7, were able to contribute to increasing the chondrogenic potential of progenitor cells as well. Moreover, BMP-2 enables the generation of hypertrophic cartilage whereas BMP-7 prevented chondrocyte hypertrophy. Furthermore, as an alternative approach to optimize chondrogenic differentiation we found that increasing the osmolarity of the culture medium to physiological levels found in mature articular cartilage was beneficial, and that a Nfat5 - Sox 9 genetic interaction is involved in this process.

Equally important for optimal chondrogenic differentiation is not only to stimulate the progenitor cells towards chondrocytes but also keeping them chondrocytes and prevent them from hypertrophy. In this manuscript we found several novel approaches in suppressing chondrocyte hypertrophy during chondrogenic differentiation of progenitor 
cells. Interestingly, we found that BMP-7 was able to suppress the chondrocyte hypertrophy via regulation of Bapx1/Nkx3.2. We identified a role for COX-2 in hypertrophy as inhibition of COX-2 resulted in suppressed hypertrophy while leaving chondrogenic differentiation unaltered. Also, we determined how the prostaglandin synthesizing enzyme COX-2 might influence chondrogenic differentiation and how this relates to the other prostaglandin synthesizing enzyme COX-1.

For cartilage regenerative approaches one could envision a procedure where progenitor cells are per-operatively isolated and pre-treated for a short period of time with LPS/TNF $\alpha$ / growth factors to induce inflammatory signaling, priming the cells for chondrogenic differentiation before transplanting using a selected cartilage repair technique. Part of the failure of current cartilage repair techniques originates from calcification or hypertrophic differentiation of the cartilage graft, as well due to the development of interlesional osteophytes. These adverse effects might be avoided when joint homeostasis is taken into account as an important factor in the post-operative treatment strategy after cartilage repair. A synovial fluid environment supplemented with the aforementioned factors (increased osmolarity, COX-inhibitors, BMP-7) might contribute to the success rate on an anti-hypertrophic basis. To be able to test the above candidates on human articular chondrocytes in a hypertrophic differentiation context in the future, we set up a human in vitro culture system suitable of displaying hypertrophic potential. Using this $2 \mathrm{D}$ model requires protein analysis as an important read out parameter and once again stresses on the mutual analysis of mRNA and protein in chondrocyte molecular biology.

Creating cartilage or bone by triggering endochondral ossification in an ectopical site and using the body as its own "in situ incubator" also has many advantages. The cells provide their own matrix and complex and costly isolation; selection and culturing procedures are bypassed. Optimization of the by our group described in vivo bioreactor technique ${ }^{90}$ might also benefit from the aforementioned approaches in both stimulating progenitor cell differentiation (treatment with LPS/TNF $\alpha /$ growth factors or increasing osmolarity) and prevention of hypertrophy (COX-2 inhibition/BMP-7 supplementation).

Taken together, our findings described in this thesis on molecular pathways involved in regulating the chondrogenic phase of endochondral ossification may not only be a logical way to stimulate cartilage (and endochondral bone) (re)generation, but is also expected to provide useful information how to keep differentiating progenitors or mature chondrocytes in the desired phase and will contribute to our understanding of skeletal developmental processes, fracture healing and diseases like OA. 


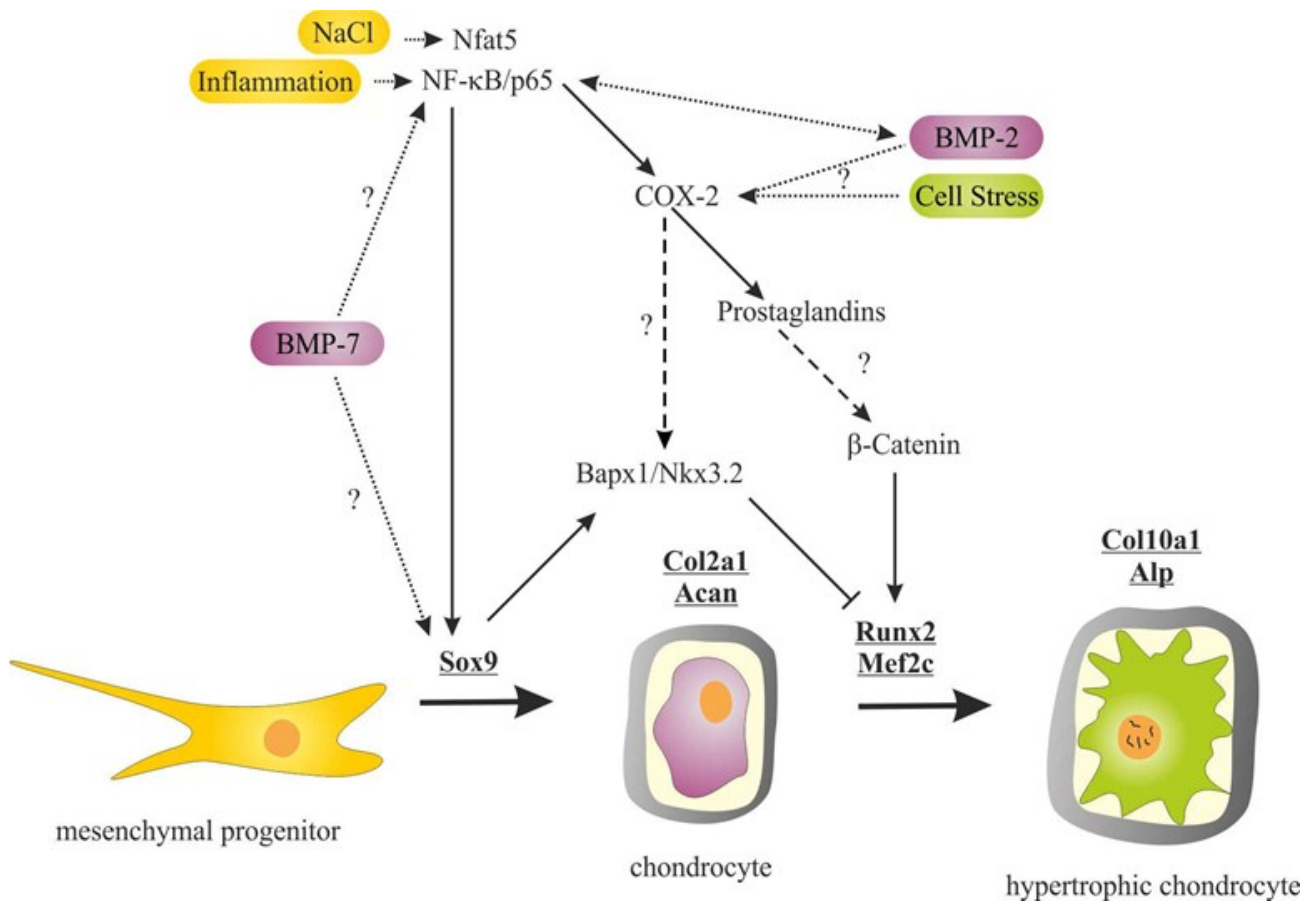

Fig 8.3 Overview of regulators studied in this thesis during chondrogenic differentiation.

During endochondral ossification mesenchymal progenitor cells differentiate towards chondrocytes which eventually become hypertrophic chondrocytes. These phases can be marked by specific genes; Sox9 for early chondrogenic differentiation, Col2a1 and Acan for mature chondrocytes and Runx2, Mef2c, Col10a1 and Alp for hypertrophic differentiation. In Chapter 2 we have shown that (mild) inflammatory stimuli can activate the NF$\kappa \mathrm{B} / \mathrm{p} 65$ signalling and subsequent induction of Sox 9 transcription which results in increased chondrogenic outcome. In Chapter 3 we determined that a similar pathway was activated by Nfat 5 when the progenitor cells were exposed to increased osmolarity of the cellular environment. NF- $\mathrm{kB} / \mathrm{p} 65$ signalling not only resulted in increased Sox9 expression but also in increased COX-2 enzyme activity. The role for COX-2 in chondrogenic differentiation was determined in Chapter 5 and 6 and revealed a COX-2 specific role in hypertrophic differentiation possibly signalling via $\beta$-Catenin. As COX-2 is a prostaglandin synthesizing enzyme a start was also made by determining the specific effect of each prostaglandin subclass on chondrogenic outcome and the possible underlying molecular mechanisms (Chapter 6). COX-2 inhibition might thus be an interesting pharmaceutical approach in inhibiting chondrocyte hypertrophy. In Chapter 4 a differential effect of BMP-2 and BMP-7 on chondrogenic outcome was described, with a more pronounced stimulating effect on hypertrophic differentiation for BMP-2 whereas BMP-7 stimulation had a chondrocyte hypertrophy suppressing effect. For BMP-7 a potential underlying mechanism was found in Bapx1/Nkx3.2 signalling which could be driven via early Sox 9 transcriptional activation and which results in a suppression of Runx2 and subsequent hypertropic differentiation. BMP-2 resulted in a decreased Bapx1/Nkx3.2 signalling which could explain the increased hypertrophic differentiation in this context. It remains elusive if BMP-2 signals via COX-2 in regulating hypertrophic differentiation, directly via Bapx1/ Nkx3.2 or via alternative pathways (e.g. $\beta$-Catenin directly). Taken together, our findings described in this thesis on molecular pathways involved in regulating the chondrogenic phase of endochondral ossification is expected to provide useful information on how to differentiate progenitor cells and to keep differentiating progenitors or mature chondrocytes in the desired phase. 
4. References

1 Bondeson, J. et al. The role of synovial macrophages and macrophage-produced mediators in driving inflammatory and destructive responses in osteoarthritis. Arthritis and rheumatism 62, 647-657, doi:10.1002/art.27290 (2010).

2 Sofat, N. Analysing the role of endogenous matrix molecules in the development of osteoarthritis. Int $J$ Exp Pathol 90, 463-479, doi:IEP676 [pii]10.1111/j.13652613.2009.00676.x (2009).

3 Pelletier, J. P. \& Martel-Pelletier, J. The Novartis-ILAR Rheumatology Prize 2001 Osteoarthritis: from molecule to man. Arthritis Res 4, 13-19 (2002).

4 Gelse, K., Soder, S., Eger, W., Diemtar, T. \& Aigner, T. Osteophyte development --molecular characterization of differentiation stages. Osteoarthritis and cartilage / OARS, Osteoarthritis Research Society 11, 141-148 (2003).

5 van der Kraan, P. M. \& van den Berg, W. B. Osteophytes: relevance and biology. Osteoarthritis Cartilage 15, 237-244, doi:S1063-4584(06)00327-X [pii]10.1016/ j.joca.2006.11.006 (2007).

6 Einhorn, T. A. The science of fracture healing. Journal of orthopaedic trauma 19, S4-6 (2005).

7 Gerstenfeld, L. C., Cullinane, D. M., Barnes, G. L., Graves, D. T. \& Einhorn, T. A. Fracture healing as a post-natal developmental process: molecular, spatial, and temporal aspects of its regulation. Journal of cellular biochemistry 88, 873-884 (2003).

8 Grundnes, O. \& Reikeras, O. The importance of the hematoma for fracture healing in rats. Acta Orthop Scand 64, 340-342 (1993).

9 Caron, M. M. et al. Activation of NF-kappaB/p65 Facilitates Early Chondrogenic Differentiation during Endochondral Ossification. PLoS One 7, e33467, doi:10.1371/journal.pone.0033467 (2012).

10 Aung, A., Gupta, G., Majid, G. \& Varghese, S. Osteoarthritic chondrocytesecreted morphogens induce chondrogenic differentiation of human mesenchymal stem cells. Arthritis Rheum 63, 148-158, doi:10.1002/art.30086 (2011).

11 Chen, C. C. et al. Cartilage fragments from osteoarthritic knee promote chondrogenesis of mesenchymal stem cells without exogenous growth factor induction. $J$ Orthop Res 30, 393-400, doi:10.1002/jor.21541 (2012).

12 Grau, A. M., Datta, P. K., Zi, J., Halder, S. K. \& Beauchamp, R. D. Role of Smad proteins in the regulation of NF-kappaB by TGF-beta in colon cancer cells. Cell Signal 18, 1041-1050, doi:S0898-6568(05)00235-4 [pii]10.1016/ j.cellsig.2005.08.021 (2006).

13 Wu, S., Fadoju, D., Rezvani, G. \& De Luca, F. Stimulatory effects of insulin-like growth factor-I on growth plate chondrogenesis are mediated by nuclear factorkappaB p65. J Biol Chem 283, 34037-34044 (2008).

14 Sugimori, K. et al. BMP-2 prevents apoptosis of the N1511 chondrocytic cell line through PI3K/Akt-mediated NF-kappaB activation. J Bone Miner Metab 23, 411419, doi:10.1007/s00774-005-0622-7 (2005).

15 Park, M. et al. Constitutive RelA activation mediated by Nkx3.2 controls chondrocyte viability. Nat Cell Biol 9, 287-298 (2007).

16 Zeng, L., Kempf, H., Murtaugh, L. C., Sato, M. E. \& Lassar, A. B. Shh establishes an Nkx3.2/Sox9 autoregulatory loop that is maintained by BMP signals to in- 
duce somitic chondrogenesis. Genes Dev 16, 1990-2005, doi:10.1101/ gad.1008002 (2002).

17 Crisostomo, P. R. et al. Human mesenchymal stem cells stimulated by TNFalpha, LPS, or hypoxia produce growth factors by an NF kappa B- but not JNKdependent mechanism. American journal of physiology 294, C675-682 (2008).

18 Einhorn, T. A., Majeska, R. J., Rush, E. B., Levine, P. M. \& Horowitz, M. C. The expression of cytokine activity by fracture callus. J Bone Miner Res 10, 12721281, doi:10.1002/jbmr.5650100818 (1995).

19 Mountziaris, P. M. \& Mikos, A. G. Modulation of the Inflammatory Response for Enhanced Bone Tissue Regeneration. Tissue Eng Part B Rev (2008).

20 Hunziker, E. B., Kapfinger, E. \& Geiss, J. The structural architecture of adult mammalian articular cartilage evolves by a synchronized process of tissue resorption and neoformation during postnatal development. Osteoarthritis Cartilage 15, 403-413, doi:S1063-4584(06)00275-5 [pii]10.1016/j.joca.2006.09.010 (2007).

21 Schenk, R., Eggli, P. \& Hunziker, E. Articular cartilage morphology. (Raven Press, 1986).

22 Schipani, E. Hypoxia and HIF-1 alpha in chondrogenesis. Semin Cell Dev Biol 16, 539-546, doi:S1084-9521(05)00057-1 [pii]10.1016/j.semcdb.2005.03.003 (2005).

23 Amarilio, R. et al. HIF1alpha regulation of Sox9 is necessary to maintain differentiation of hypoxic prechondrogenic cells during early skeletogenesis. Development 134, 3917-3928, doi:dev.008441 [pii]10.1242/dev.008441 (2007).

24 Hirao, M., Tamai, N., Tsumaki, N., Yoshikawa, H. \& Myoui, A. Oxygen tension regulates chondrocyte differentiation and function during endochondral ossification. J Biol Chem 281, 31079-31092, doi:M602296200 [pii]10.1074/ jbc.M602296200 (2006).

25 Urban, J. P. The chondrocyte: a cell under pressure. Br J Rheumatol 33, 901-908 (1994).

26 Urban, J. P., Hall, A. C. \& Gehl, K. A. Regulation of matrix synthesis rates by the ionic and osmotic environment of articular chondrocytes. J Cell Physiol 154, 262270, doi:10.1002/jcp.1041540208 (1993).

27 Lesperance, L. M., Gray, M. L. \& Burstein, D. Determination of fixed charge density in cartilage using nuclear magnetic resonance. J Orthop Res 10, 1-13, doi:10.1002/jor.1100100102 (1992).

28 Venn, M. \& Maroudas, A. Chemical composition and swelling of normal and osteoarthrotic femoral head cartilage. I. Chemical composition. Annals of the rheumatic diseases 36, 121-129 (1977).

29 Palmer, G. D. et al. Time-dependent aggrecan gene expression of articular chondrocytes in response to hyperosmotic loading. Osteoarthritis Cartilage 9, 761770, doi:10.1053/joca.2001.0473S106345840190473X [pii] (2001).

30 van der Windt, A. E. et al. Physiological tonicity improves human chondrogenic marker expression through nuclear factor of activated T-cells 5 in vitro. Arthritis research \& therapy 12, R100, doi:10.1186/ar3031 (2010).

31 van der Windt, A. E. et al. Inhibiting calcineurin activity under physiological tonicity elevates anabolic but suppresses catabolic chondrocyte markers. Arthritis and rheumatism, doi:10.1002/art.34369 (2012).

32 Maroudas, A. I. Balance between swelling pressure and collagen tension in normal and degenerate cartilage. Nature 260, 808-809 (1976).

33 Bank, R. A., Soudry, M., Maroudas, A., Mizrahi, J. \& TeKoppele, J. M. The increased swelling and instantaneous deformation of osteoarthritic cartilage is highly correlated with collagen degradation. Arthritis Rheum 43, 2202-2210, doi:10.1002/1529-0131(200010)43:10<2202::AID-ANR7>3.0.CO;2-E (2000). 
Halterman, J. A., Kwon, H. M. \& Wamhoff, B. R. Tonicity-independent regulation of the osmosensitive transcription factor TonEBP (NFAT5). American journal of physiology. Cell physiology 302, C1-8, doi:10.1152/ajpcell.00327.2011 (2012).

35 Yoon, H. J. et al. NFAT5 is a critical regulator of inflammatory arthritis. Arthritis and rheumatism, doi:10.1002/art.30229 (2011).

36 Jauliac, S. et al. The role of NFAT transcription factors in integrin-mediated carcinoma invasion. Nat Cell Biol 4, 540-544, doi:10.1038/ncb816 (2002). Colter, D. C. et al. Regulation of the human Sox9 promoter by the CCAATbinding factor. Matrix biology : journal of the International Society for Matrix Biology 24, 185-197, doi:10.1016/j.matbio.2005.04.001 (2005).

Piera-Velazquez, S. et al. Regulation of the human SOX9 promoter by Sp1 and CREB. Experimental cell research 313, 1069-1079, doi:10.1016/ j.yexcr.2007.01.001 (2007).

Akiyama, H., Chaboissier, M. C., Martin, J. F., Schedl, A. \& de Crombrugghe, B. The transcription factor Sox9 has essential roles in successive steps of the chondrocyte differentiation pathway and is required for expression of Sox 5 and Sox6. Genes Dev 16, 2813-2828 (2002).

40 Han, Y. \& Lefebvre, V. L-Sox 5 and Sox6 drive expression of the aggrecan gene in cartilage by securing binding of Sox9 to a far-upstream enhancer. Mol Cell Biol 28, 4999-5013, doi:MCB.00695-08 [pii]10.1128/MCB.00695-08 (2008).

41 Lefebvre, V., Behringer, R. R. \& de Crombrugghe, B. L-Sox5, Sox6 and Sox9 control essential steps of the chondrocyte differentiation pathway. Osteoarthritis Cartilage 9 Suppl A, S69-75 (2001).

Lefebvre, V., Huang, W., Harley, V. R., Goodfellow, P. N. \& de Crombrugghe, B. SOX9 is a potent activator of the chondrocyte-specific enhancer of the pro alpha1(II) collagen gene. Mol Cell Biol 17, 2336-2346 (1997).

Lefebvre, V., Li, P. \& de Crombrugghe, B. A new long form of Sox5 (L-Sox5), Sox6 and Sox 9 are coexpressed in chondrogenesis and cooperatively activate the type II collagen gene. Embo $J$ 17, 5718-5733, doi:10.1093/emboj/17.19.5718 (1998).

44 Genzer, M. A. \& Bridgewater, L. C. A Col9a1 enhancer element activated by two interdependent SOX9 dimers. Nucleic acids research 35, 1178-1186, doi:10.1093/ nar/gkm014 (2007).

45 Jenkins, E., Moss, J. B., Pace, J. M. \& Bridgewater, L. C. The new collagen gene COL27A1 contains SOX9-responsive enhancer elements. Matrix biology : journal of the International Society for Matrix Biology 24, 177-184, doi:10.1016/ j.matbio.2005.02.004 (2005).

46 Rentsendorj, O. et al. Highly conserved proximal promoter element harbouring paired Sox9-binding sites contributes to the tissue- and developmental stagespecific activity of the matrilin-1 gene. Biochem $J$ 389, 705-716, doi:10.1042/ BJ20050214 (2005).

47 Oh, C. D. et al. Identification of SOX9 interaction sites in the genome of chondrocytes. PLoS One 5, e10113, doi:10.1371/journal.pone.0010113 (2010).

48 Welting, T. J. et al. Inhibition of cyclooxygenase-2 impacts chondrocyte hypertrophic differentiation during endochondral ossification. European cells \& materials 22, 420-436; discussion 436-427 (2011).

49 Caron, M. M. et al. Redifferentiation of dedifferentiated human articular chondrocytes: comparison of 2D and 3D cultures. Osteoarthritis and cartilage / OARS, Osteoarthritis Research Society 20, 1170-1178, doi:10.1016/j.joca.2012.06.016 (2012). 
50 Lengner, C. J. et al. Nkx3.2-mediated repression of Runx2 promotes chondrogenic differentiation. The Journal of biological chemistry 280, 15872-15879, doi:10.1074/jbc.M411144200 (2005).

51 Provot, S. et al. Nkx3.2/Bapx1 acts as a negative regulator of chondrocyte maturation. Development 133, 651-662, doi:10.1242/dev.02258 (2006).

52 Yamashita, S. et al. Sox9 directly promotes Bapx1 gene expression to repress Runx2 in chondrocytes. Experimental cell research 315, 2231-2240, doi:10.1016/ j.yexcr.2009.03.008 (2009).

53 Zhou, G. et al. Dominance of SOX9 function over RUNX2 during skeletogenesis. Proc Natl Acad Sci U S A 103, 19004-19009 (2006).

54 Murtaugh, L. C., Zeng, L., Chyung, J. H. \& Lassar, A. B. The chick transcriptional repressor Nkx3.2 acts downstream of Shh to promote BMP-dependent axial chondrogenesis. Dev Cell 1, 411-422 (2001).

55 Kawato, Y. et al. Nkx3.2 promotes primary chondrogenic differentiation by upregulating Col2al transcription. PLoS One 7, e34703, doi:10.1371/ journal.pone.0034703 (2012).

56 Yong, Y. et al. Exogenous signal-independent nuclear IkappaB kinase activation triggered by Nkx3.2 enables constitutive nuclear degradation of IkappaB-alpha in chondrocytes. Molecular and cellular biology 31, 2802-2816, doi:10.1128/ MCB.00253-10 (2011).

57 Miyazono, K., Maeda, S. \& Imamura, T. BMP receptor signaling: transcriptional targets, regulation of signals, and signaling cross-talk. Cytokine Growth Factor Rev 16, 251-263, doi:S1359-6101(05)00039-0 [pii]10.1016/j.cytogfr.2005.01.009 (2005).

58 Pogue, R. \& Lyons, K. BMP signaling in the cartilage growth plate. Curr Top Dev Biol 76, 1-48, doi:10.1016/S0070-2153(06)76001-X (2006).

59 Zhang, D. et al. ALK2 functions as a BMP type I receptor and induces Indian hedgehog in chondrocytes during skeletal development. Journal of bone and mineral research : the official journal of the American Society for Bone and Mineral Research 18, 1593-1604, doi:10.1359/jbmr.2003.18.9.1593 (2003).

60 Zou, H., Wieser, R., Massague, J. \& Niswander, L. Distinct roles of type I bone morphogenetic protein receptors in the formation and differentiation of cartilage. Genes Dev 11, 2191-2203 (1997).

61 Hellingman, C. A. et al. Smad Signaling Determines Chondrogenic Differentiation of Bone-Marrow-Derived Mesenchymal Stem Cells: Inhibition of Smad1/5/8P Prevents Terminal Differentiation and Calcification. Tissue Eng Part $A$, doi:10.1089/ten.TEA.2010.0043 (2011).

62 Kamekura, S. et al. Contribution of runt-related transcription factor 2 to the pathogenesis of osteoarthritis in mice after induction of knee joint instability. Arthritis Rheum 54, 2462-2470 (2006).

63 Kawaguchi, H. Endochondral ossification signals in cartilage degradation during osteoarthritis progression in experimental mouse models. Mol Cells 25, 1-6 (2008).

64 Saito, T. et al. Transcriptional regulation of endochondral ossification by HIF2alpha during skeletal growth and osteoarthritis development. Nat Med 16, 678686, doi:nm.2146 [pii]10.1038/nm.2146 (2010).

65 van der Kraan, P. M. \& van den Berg, W. B. Chondrocyte hypertrophy and osteoarthritis: role in initiation and progression of cartilage degeneration? Osteoarthritis and cartilage / OARS, Osteoarthritis Research Society 20, 223-232, doi:10.1016/j.joca.2011.12.003 (2012).

66 Zuscik, M. J., Hilton, M. J., Zhang, X., Chen, D. \& O'Keefe, R. J. Regulation of 
chondrogenesis and chondrocyte differentiation by stress. J Clin Invest 118, 429438 (2008).

67 Henrotin, Y., Kurz, B. \& Aigner, T. Oxygen and reactive oxygen species in cartilage degradation: friends or foes? Osteoarthritis Cartilage 13, 643-654, doi:S1063 -4584(05)00098-1 [pii]10.1016/j.joca.2005.04.002 (2005).

68 Jallali, N., Ridha, H., Thrasivoulou, C., Butler, P. \& Cowen, T. Modulation of intracellular reactive oxygen species level in chondrocytes by IGF-1, FGF, and TGF-beta1. Connect Tissue Res 48, 149-158, doi:779019649 [pii] 10.1080/03008200701331516 (2007).

69 Morita, K. et al. Reactive oxygen species induce chondrocyte hypertrophy in endochondral ossification. J Exp Med 204, 1613-1623, doi:jem.20062525 [pii] 10.1084/jem.20062525 (2007).

70 Cecil, D. L. et al. Inflammation-induced chondrocyte hypertrophy is driven by receptor for advanced glycation end products. J Immunol 175, 8296-8302, doi:175/12/8296 [pii] (2005).

71 Handl, M. et al. Fluorescent advanced glycation end products in the detection of factual stages of cartilage degeneration. Physiol Res 56, 235-242, doi:934 [pii] (2007).

72 Huang, C. Y., Hung, L. F., Liang, C. C. \& Ho, L. J. COX-2 and iNOS are critical in advanced glycation end product-activated chondrocytes in vitro. Eur J Clin Invest 39, 417-428, doi:ECI2106 [pii]10.1111/j.1365-2362.2009.02106.x (2009).

73 Kume, S. et al. Advanced glycation end-products attenuate human mesenchymal stem cells and prevent cognate differentiation into adipose tissue, cartilage, and bone. J Bone Miner Res 20, 1647-1658, doi:10.1359/JBMR.050514 (2005).

74 Nah, S. S. et al. Effects of advanced glycation end products on the expression of COX-2, PGE2 and NO in human osteoarthritic chondrocytes. Rheumatology (Oxford) 47, 425-431, doi:kem376 [pii]10.1093/rheumatology/kem376 (2008).

75 Arasapam, G., Scherer, M., Cool, J. C., Foster, B. K. \& Xian, C. J. Roles of COX2 and iNOS in the bony repair of the injured growth plate cartilage. Journal of cellular biochemistry 99, 450-461 (2006).

76 Baldik, Y. et al. Deletion of iNOS gene impairs mouse fracture healing. Bone 37, 32-36 (2005).

77 Mais, A., Klein, T., Ullrich, V., Schudt, C. \& Lauer, G. Prostanoid pattern and iNOS expression during chondrogenic differentiation of human mesenchymal stem cells. Journal of cellular biochemistry 98, 798-809 (2006).

78 Itoh, S. S., M. Ikeda, T. Yano, F. Ogata, N. Chung, U. Nakamura, K. Kawaguchi, H. NF-kappa B family member RelA/p65, a transcription factor of Sox9, is essential for chondrogenic differentiation and skeletal growth. Osteoarthritis and Cartilage 17, S12 - S13 (2009).

79 Wu, S., Flint, J. K., Rezvani, G. \& De Luca, F. Nuclear factor-kappaB p65 facilitates longitudinal bone growth by inducing growth plate chondrocyte proliferation and differentiation and by preventing apoptosis. J Biol Chem 282, 33698-33706 (2007).

80 Kishimoto, H. et al. Induction of hypertrophic chondrocyte-like phenotypes by oxidized LDL in cultured bovine articular chondrocytes through increase in oxidative stress. Osteoarthritis and cartilage / OARS, Osteoarthritis Research Society 18, 1284-1290, doi:10.1016/j.joca.2010.05.021 (2010).

81 Nakagawa, S. et al. N-acetylcysteine prevents nitric oxide-induced chondrocyte apoptosis and cartilage degeneration in an experimental model of osteoarthritis. $J$ Orthop Res 28, 156-163, doi:10.1002/jor.20976 (2010).

82 Roman-Blas, J. A. et al. Differential effects of the antioxidant n-acetylcysteine on 
the production of catabolic mediators in IL-1beta-stimulated human osteoarthritic synoviocytes and chondrocytes. European journal of pharmacology 623, 125-131, doi:10.1016/j.ejphar.2009.09.016 (2009).

83 Csaki, C., Keshishzadeh, N., Fischer, K. \& Shakibaei, M. Regulation of inflammation signalling by resveratrol in human chondrocytes in vitro. Biochem Pharmacol 75, 677-687, doi:10.1016/j.bcp.2007.09.014 (2008).

84 Liu, F. C. et al. Chondroprotective effects and mechanisms of resveratrol in advanced glycation end products-stimulated chondrocytes. Arthritis research \& therapy 12, R167, doi:10.1186/ar3127 (2010).

85 Shakibaei, M., Csaki, C., Nebrich, S. \& Mobasheri, A. Resveratrol suppresses interleukin-1beta-induced inflammatory signaling and apoptosis in human articular chondrocytes: potential for use as a novel nutraceutical for the treatment of osteoarthritis. Biochem Pharmacol 76, 1426-1439, doi:10.1016/j.bcp.2008.05.029 (2008).

86 Chowdhury, T. T., Bader, D. L. \& Lee, D. A. Dynamic compression counteracts IL-1beta induced iNOS and COX-2 activity by human chondrocytes cultured in agarose constructs. Biorheology 43, 413-429 (2006).

87 Dickhut, A. et al. Calcification or dedifferentiation: requirement to lock mesenchymal stem cells in a desired differentiation stage. J Cell Physiol 219, 219-226 (2009).

88 Fischer, J., Dickhut, A., Rickert, M. \& Richter, W. Human articular chondrocytes secrete parathyroid hormone-related protein and inhibit hypertrophy of mesenchymal stem cells in coculture during chondrogenesis. Arthritis Rheum 62, 26962706, doi:10.1002/art.27565 (2010).

89 Chikazu, D. et al. Bone morphogenetic protein 2 induces cyclo-oxygenase 2 in osteoblasts via a Cbfal binding site: role in effects of bone morphogenetic protein 2 in vitro and in vivo. J Bone Miner Res 17, 1430-1440 (2002).

90 Emans, P. J. et al. Autologous engineering of cartilage. Proc Natl Acad Sci U S A 107, 3418-3423, doi:0907774107 [pii]10.1073/pnas.0907774107 (2010).

91 Thaller, J., Walker, M., Kline, A. J. \& Anderson, D. G. The effect of nonsteroidal anti-inflammatory agents on spinal fusion. Orthopedics 28, 299-303; quiz 304295 (2005).

92 Gerstenfeld, L. C. et al. Differential inhibition of fracture healing by non-selective and cyclooxygenase-2 selective non-steroidal anti-inflammatory drugs. J Orthop Res 21, 670-675 (2003).

93 Herbenick, M. A., Sprott, D., Stills, H. \& Lawless, M. Effects of a cyclooxygenase 2 inhibitor on fracture healing in a rat model. Am J Orthop (Belle Mead NJ) 37, E133-137 (2008).

94 Goodman, S. et al. COX-2 selective NSAID decreases bone ingrowth in vivo. $J$ Orthop Res 20, 1164-1169 (2002).

95 Simon, A. M. \& O'Connor, J. P. Dose and time-dependent effects of cyclooxygenase-2 inhibition on fracture-healing. J Bone Joint Surg Am 89, 500-511 (2007).

96 Grohs, J. G., Schmidt, M. \& Wanivenhaus, A. Selective COX-2 inhibitor versus indomethacin for the prevention of heterotopic ossification after hip replacement: a double-blind randomized trial of 100 patients with 1-year follow-up. Acta Orthop 78, 95-98 (2007).

97 Funk, C. D. Prostaglandins and leukotrienes: advances in eicosanoid biology. Science 294, 1871-1875, doi:10.1126/science.294.5548.1871 (2001).

98 Ouyang, P. An in vitro model to study mesenchymal-epithelial transformation. Biochemical and biophysical research communications 246, 771-776, doi:10.1006/bbrc.1998.8710 (1998). 
99 Goldring, M. B. et al. Interleukin-1 beta-modulated gene expression in immortalized human chondrocytes. J Clin Invest 94, 2307-2316, doi:10.1172/JCI117595 (1994).

100 Liang, Z. J. et al. MiRNA-140 is a negative feedback regulator of MMP-13 in IL1beta-stimulated human articular chondrocyte C28/I2 cells. Inflamm Res 61, 503509, doi:10.1007/s00011-012-0438-6 (2012).

101 Atsumi, T., Miwa, Y., Kimata, K. \& Ikawa, Y. A chondrogenic cell line derived from a differentiating culture of AT805 teratocarcinoma cells. Cell Differ Dev 30, 109-116 (1990).

102 Denker, A. E., Haas, A. R., Nicoll, S. B. \& Tuan, R. S. Chondrogenic differentiation of murine C3H10T1/2 multipotential mesenchymal cells: I. Stimulation by bone morphogenetic protein-2 in high-density micromass cultures. Differentiation 64, 67-76 (1999).

103 Zehentner, B. K., Dony, C. \& Burtscher, H. The transcription factor Sox9 is involved in BMP-2 signaling. Journal of bone and mineral research : the official journal of the American Society for Bone and Mineral Research 14, 1734-1741, doi:10.1359/jbmr.1999.14.10.1734 (1999).

104 Yagami, K., Kakuta, S., Tachibana, H., Kimura, Y. \& Nagumo, M. Establishment of a cell line with phenotypes of chondrocyte from a human osteogenic sarcoma of the mandible. J Oral Pathol Med 29, 321-330 (2000).

105 Yagami, K. et al. A human chondrogenic cell line retains multi-potency that differentiates into osteoblasts and adipocytes. Bone 34, 648-655, doi:10.1016/ j.bone.2003.12.016S8756328203004575 [pii] (2004).

106 Lefebvre, V., Garofalo, S. \& de Crombrugghe, B. Type X collagen gene expression in mouse chondrocytes immortalized by a temperature-sensitive simian virus 40 large tumor antigen. J Cell Biol 128, 239-245 (1995).

107 Saito, T. \& Kawaguchi, H. HIF-2alpha as a possible therapeutic target of osteoarthritis. Osteoarthritis Cartilage 18, 1552-1556, doi:S1063-4584(10)00336-5 [pii] 10.1016/j.joca.2010.10.006.

108 Knippenberg, M., Helder, M. N., Zandieh Doulabi, B., Wuisman, P. I. \& KleinNulend, J. Osteogenesis versus chondrogenesis by BMP-2 and BMP-7 in adipose stem cells. Biochemical and biophysical research communications 342, 902-908, doi:10.1016/j.bbrc.2006.02.052 (2006). 


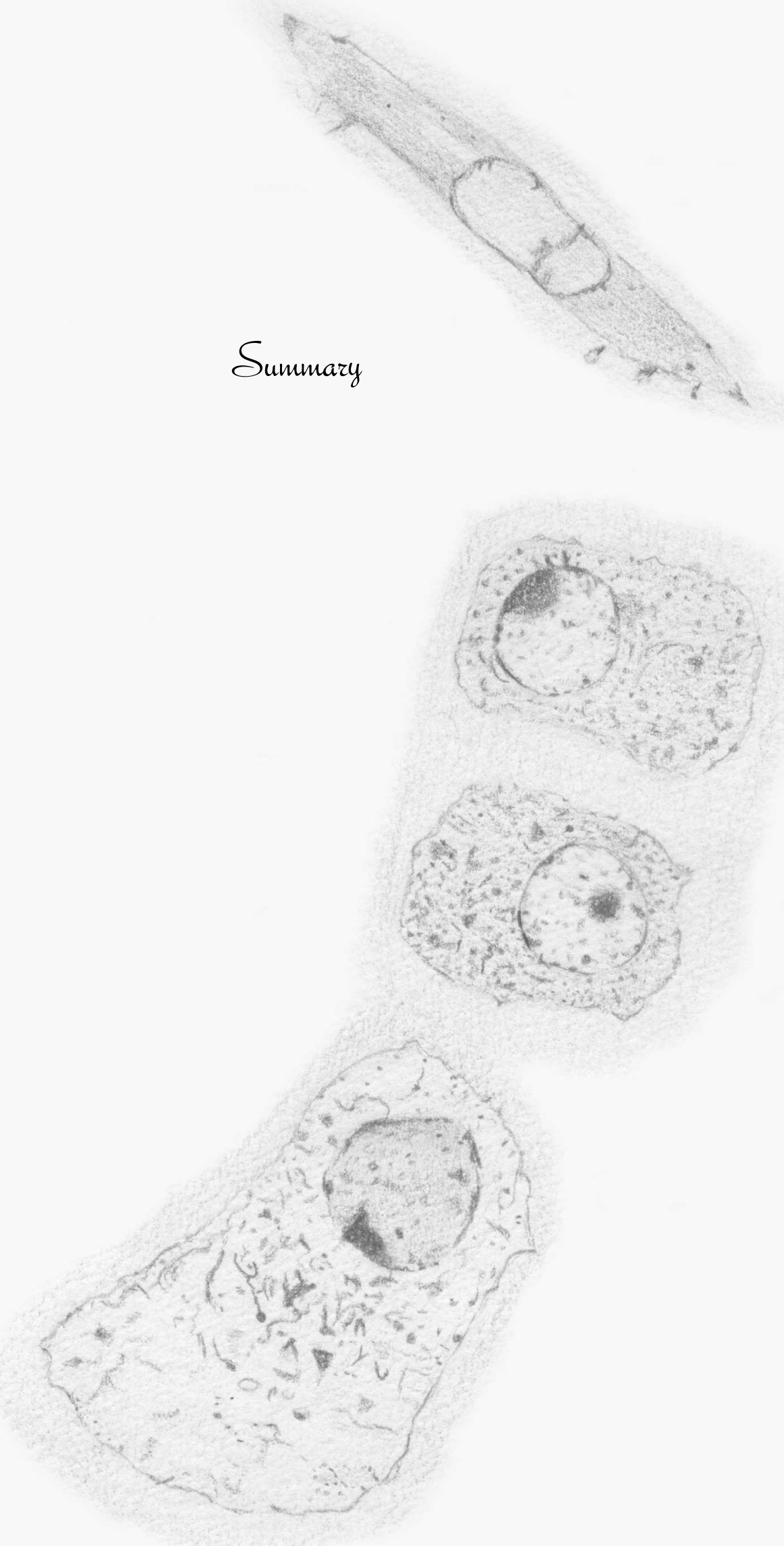

Chondrogenic differentiation is a tightly regulated process in which many factors regulate the discrete stages of the differentiation program. Understanding the mechanisms of cell commitment and further differentiation into the chondrogenic lineage is of great importance to optimize (progenitor) cell-based cartilage repair techniques. The work described in this thesis aimed to find novel perspectives to increase the chondrogenic differentiation capacity from progenitor cells and modulate their chondrogenic outcome.

Several approaches were explored to enhance the chondrogenic differentiation capacity of progenitor cells. First it was found that inflammatory mediators as NF- $\mathrm{kB} / \mathrm{p} 65$ have a role in the onset of chondrogenic differentiation from progenitor cells. Modulating the activity of NF- $\mathrm{kB} / \mathrm{p} 65$ alters the chondrogenic outcome via signalling through key chondrogenic transcription factor Sox9. In addition, expression of several growth factors, including BMP -2 , is co-regulated in differentiating chondrocytes by NF- $\mathrm{KB} / \mathrm{p} 65$ and in turn BMP-2 is able to regulate $\mathrm{NF}-\kappa \mathrm{B} / \mathrm{p} 65$ signalling which could, at least in part, explain the prochondrogenic effect of BMP-2. As an alternative approach to optimize chondrogenic differentiation from progenitor cells we found that chondrogenic marker expression of differentiating chondroprogenitor cells in vitro can be enhanced by increasing the osmolarity levels of culture medium by $100 \mathrm{mOsm}$. The osmolarity responsive gene Nfat5 is part of the mechanism that underlies this effect and might directly influence chondrogenic differentiation via controlling the expression of key chondrogenic transcription factor Sox9.

Equally important for optimal chondrogenic differentiation is not only to stimulate the progenitor cells towards chondrocytes but also keeping them chondrocytes and prevent them from hypertrophy. Several new leads were found to suppress chondrocyte hypertrophy during chondrogenic differentiation of progenitor cells. We found that BMP-2 and BMP-7 have differential effects on the chondrogenic outcome of differentiating chondroprogenitor cells: BMP-2 not only induced chondrogenic differentiation but even more chondrocyte hypertrophy, while BMP-7 appears to increase or maintain chondrogenic potential and prevent chondrocyte hypertrophy. Studies into the possible underlying mechanism explaining this discrepancy revealed Bapx1/Nkx3.2 signalling as a potential candidate in regulating chondrocyte hypertrophy. Next to BMP-7, we identified a role for COX-2 in chondrocyte hypertrophy. COX-2 is one of the main transcriptional targets of the NF- $\kappa \mathrm{B} / \mathrm{p} 65$ complex and we found that inhibition of COX -2 activity by COX -2 selective non-steroidal anti-inflammatory drugs (NSAIDs) specifically decreases the level of chondrocyte hypertrophic differentiation in different progenitor-based 
chondrogenic differentiation models, while leaving chondrogenic differentiation unaltered. Also, systemic inhibition of COX-2 activity in vivo resulted in significantly impaired chondrocyte hypertrophic differentiation in rabbit growth plates. Next to COX-2-specific NSAIDs, other NSAIDs specifically inhibit COX-1 or both COX-enzymes. It was determined that, as opposed to COX-2 inhibition, COX-1-specific NSAIDs inhibit extracellular matrix production during chondrogenic differentiation from progenitor cells. A possible explanation for the discrepancy in the chondrogenic outcome influenced by COX-1- and COX-2- specific inhibitors might lie in differential prostaglandin synthesis under these circumstances.

Especially for progenitor cell-based cartilage repair technologies, our novel insights could be employed to increase the differentiation potential of progenitor cells toward engineered cartilaginous tissue in vitro and in vivo. To also be able to test the above candidates on human articular chondrocytes in an hypertrophic differentiation context in the future, we set up a human in vitro culture system suitable of displaying hypertrophic potential. Using this $2 \mathrm{D}$ model requires protein analysis as an important read out parameter and once again stresses on the mutual analysis of mRNA and protein in chondrocyte molecular biology.

Taken together, the findings described in this thesis on molecular pathways involved in regulating the chondrogenic phase of endochondral ossification may not only be a logical way to stimulate cartilage (and endochondral bone) (re)generation, but is also expected to provide valuable information how to keep differentiating progenitor cells or mature chondrocytes in the desired differentiation phase and will contribute to our understanding of skeletal developmental processes, fracture healing and diseases like OA. 

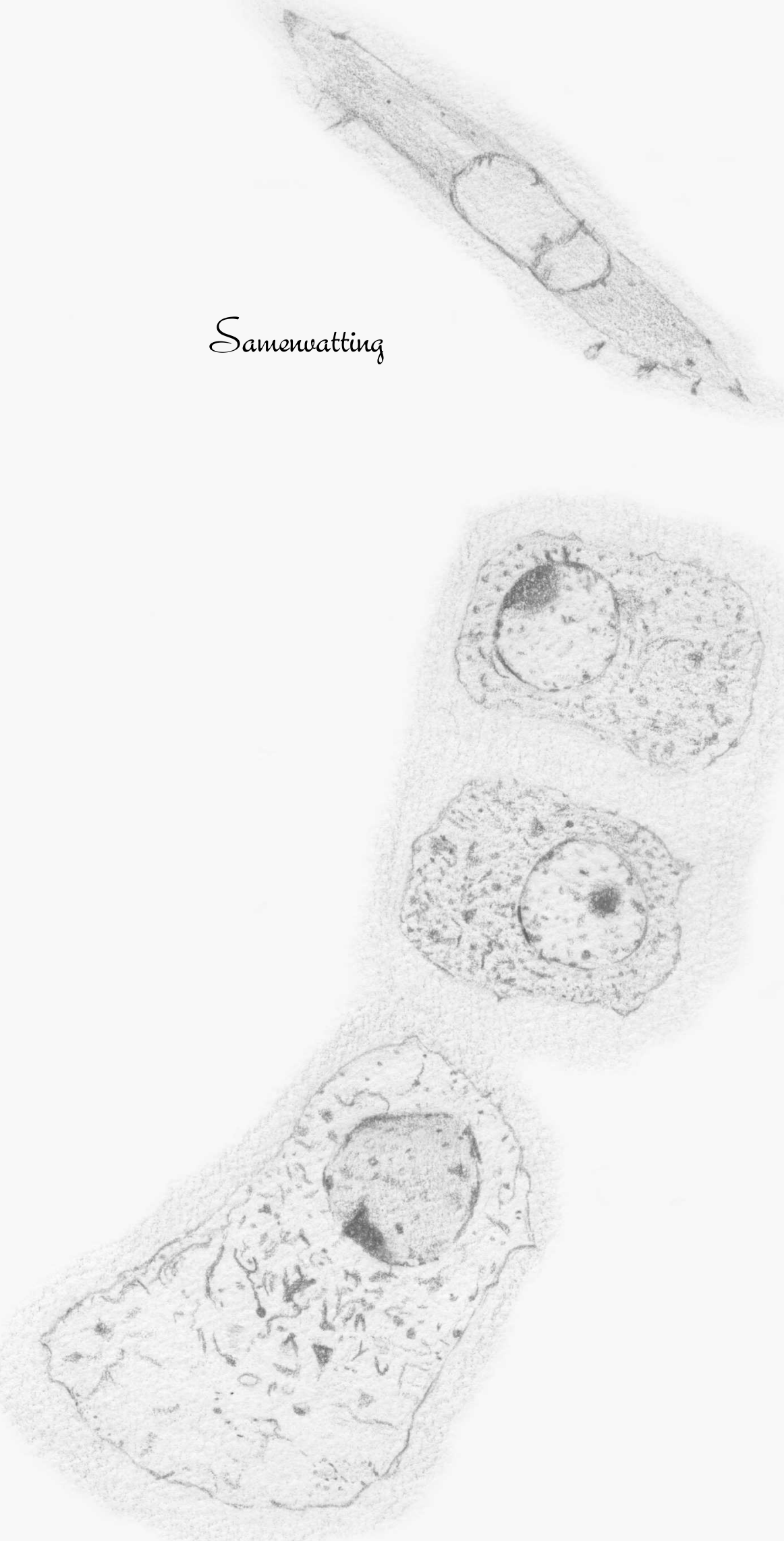

Kraakbeen differentiatie is een strak gereguleerd proces waarbij vele factoren betrokken zijn die de afzonderlijke fasen van het differentiatie programma reguleren. Het begrijpen van de moleculaire mechanismen die betrokken zijn bij de differentiatie in de chondrogene lijn zijn van groot belang voor de optimalisatie van op (voorloper/stam) cellen gebaseerde kraakbeen herstel technieken. Het werk beschreven in dit proefschrift is gericht op het vinden van nieuwe perspectieven om de chondrogene differentiatie capaciteit van kraakbeen voorloper cellen te verhogen en de chondrogene uitkomst te moduleren.

Verschillende benaderingen werden bestudeerd om de chondrogene differentiatie capaciteit van voorlopercellen te verbeteren. Allereerst hebben we vastgesteld dat ontstekings-mediatoren als NF-kB/p65 een rol spelen bij de initiatie van chondrogene dif-

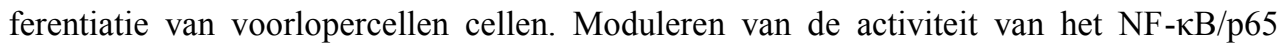
complex resulteert in een veranderde chondrogene differentiatie via signalering via een van de belangrijkste chondrogene transcriptiefactoren: Sox9. Daarnaast is de expressie van verschillende groeifactoren, waaronder BMP-2, tijdens differentiatie mede gereguleerd

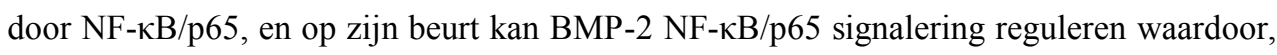
althans gedeeltelijk, het pro-chondrogene effect van BMP-2 kan worden verklaard. Als een alternatieve benadering voor de optimalisatie van chondrogene differentiatie vanuit kraakbeen voorloper cellen hebben we gevonden dat kraakbeen gen-expressie in vitro kan worden verbeterd door de osmolariteit van het kweekmedium te verhogen met $100 \mathrm{mOsm}$. $\mathrm{Nfat} 5$, het gen dat gevoelig is voor osmolariteit, makt deel uit van het mechanisme dat ten grondslag ligt dit effect en kan mogelijk chondrogene differentiatie direct beinvloeden via de het reguleren van de expressie van Sox9.

Even belangrijk voor optimale chondrogene differentiatie is niet alleen de stimulatie van kraakbeen voorloper cellen naar chondrocyten, maar ook om vervolgens het chondrocyt fenotype te behouden en kraakbeen hypertrofie te voorkomen. Verschillende nieuwe leads bleken chondrocyt hypertrofie te onderdrukken tijdens de chondrogene differentiatie van voorlopercellen. We vonden dat BMP-2 en BMP-7 differentiële effecten hebben op de uitkomst van chondrogene differentiatie chondroprogenitor cellen: BMP-2 induceerde niet alleen chondrogene differentiatie maar ook chondrocyte hypertrofie, terwijl BMP-7 het chondrogene vermogen lijkt te verhogen of handhaven en tevens chondrocyt hypertrofie te voorkomen. In de studies naar een mogelijke onderliggend mechanisme ter verklaring van deze discrepantie kwam Bapx1/Nkx3.2 signalering naar voren als een potentiële kandidaat in het reguleren van chondrocyten hypertrofie. Naast BMP-7 identificeerden we ook een rol voor COX-2 in chondrocyte hypertrofie. $\mathrm{COX}-2$ is een van de belangrijkste 
transcriptionele targets van het $\mathrm{NF}-\kappa \mathrm{B} / \mathrm{p} 65$ complex, en het bleek dat remming van COX-2 activiteit van door COX-2 selectieve niet-steroïdale anti-inflammatoire geneesmiddelen (NSAIDs) specifiek de hoogte van chondrocyte hypertrofie kon voorkomen in verschillende voorlopercel-gebaseerde chondrogene differentiatie modellen, terwijl chondrogene differentiatie ongewijzigd bleef. Ook systemische remming van COX-2 activiteit in vivo leidde tot een significante vermindering van chondrocyt hypertrofe differentiatie in groeischijven van jonge konijnen. Naast de COX-2-specifieke NSAIDs, zijn er ook andere NSAIDs die specifiek COX-1 of beide COX-enzymen remmen. Er werd vastgesteld dat, in tegenstelling tot COX-2 specifieke NSAIDs, COX-1-specifieke NSAIDs de kraakbeen extracellulaire matrix productie remmen tijdens chondrogene differentiatie van kraakbeen voorloper cellen. Een mogelijke verklaring voor deze discrepantie in de chondrogene uitkomst kan liggen in differentiële prostaglandinesynthese onder deze omstandigheden.

Speciaal voor voorlopercel-gebaseerde kraakbeenherstel technologieën kunnen onze nieuwe inzichten worden gebruikt om zo het differentiatiepotentieel van voorlopercellen te verhogen voor ge-engineered kraakbeenweefsel in vitro en in vivo. Om ook de bovengenoemde kandidaten te testen op humane articulaire chondrocyten in een hypertrofe context hebben we een in vitro differentiatie systeem opgezet voor het induceren van chondrocyt hypertrofie in humane adulte chondrocyten. Dit 2D-model vereist wel eiwit analyse als een belangrijke parameter en benadrukt zo nogmaals op het analyseren van zowel eiwit als RNA in de moleculaire chondrocyt biologie.

Tezamen kunnen de bevindingen beschreven in dit proefschrift de moleculaire pathways die betrokken zijn bij het reguleren van de chondrogene fase van endochondrale ossificatie niet alleen op een logische manier gebruikt worden om kraakbeen (en endochondrale bot) (re) generatie te stimuleren, maar zal ook naar verwachting waardevolle informatie verstrekken over hoe differentiërende stamcellen of volwassen chondrocyten in de gewenste differentiatie fase te houden en zal daarnaast bijdragen aan ons begrip van skeletaire ontwikkelingsprocessen, fractuurgenezing en ziekten zoals artrose. 


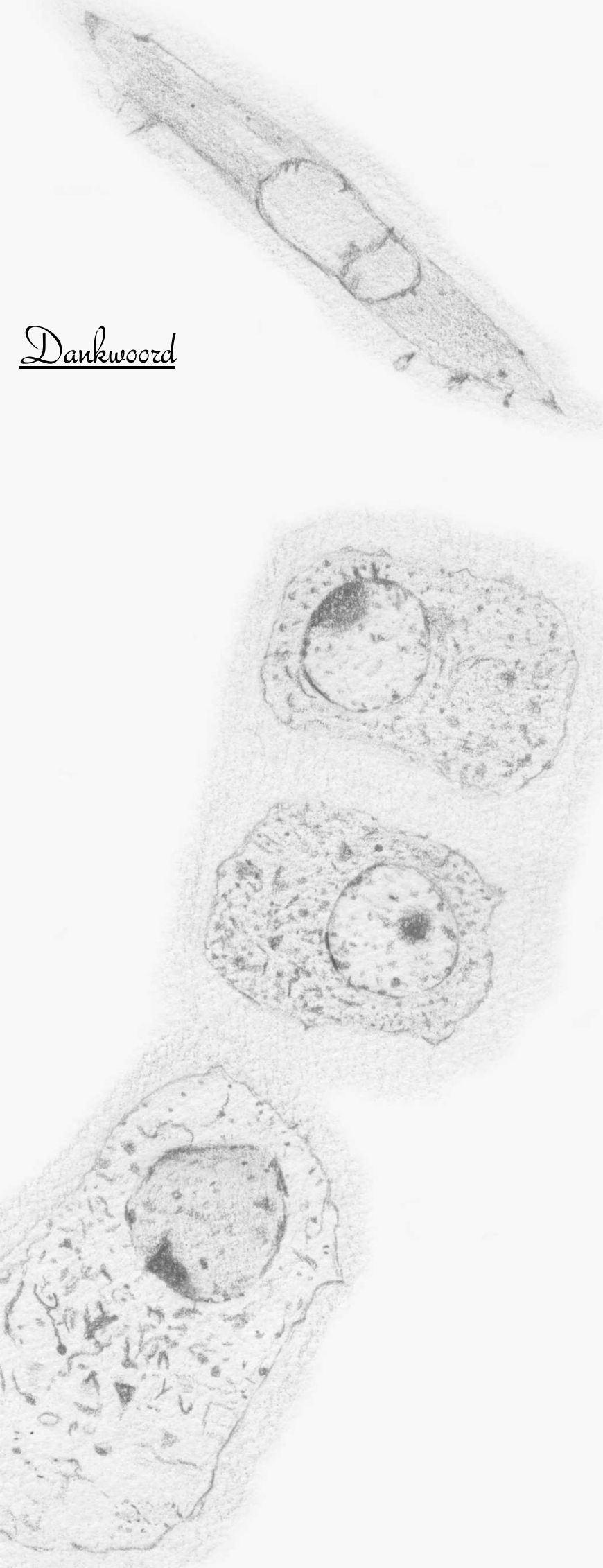



Daar is het dan, mijn proefschrift. Eindelijk! Ik denk dat dit proefschrift een mooie weerspiegeling is van wat ik de afgelopen jaren heb geleerd en hoe we samen met het laboratorium zijn gegroeid tot wat het nu is. Van een lab met alleen een centrifuge in de koelkast ('gekoeld'), een zwartgallige vortex en een golfslagbad naar een volledig ingericht laboratorium met alle proffesionele apparatuur, technieken en protocollen die we maar kunnen wensen binnen handbereik. Hopelijk gaan we zo door!

Natuurlijk heb ik dit onderzoek niet allemaal alleen gedaan en heb ik tijdens de afgelopen vier jaar hulp en sturing gehad van anderen die ik graag wil bedanken voor dit mooie resultaat.

Als allereerste mijn promotie-team: Prof. Dr. L.W. van Rhijn, Dr. T.J.M. Welting en Dr. P.J. Emans. Beste Lodewijk, als eerste wil ik je bedanken voor de kans om op deze afdeling te promoveren en voor al de adviezen en steun die je me hebt gegeven tijdens deze periode. Ik vergeet nooit meer de grote repen chocolade als troost nadat een van onze eerste 'grote' experimenten mis was gegaan.

Beste Tim, ontzettend bedankt! Als co-promotor stond je zeer dicht bij mijn onderzoek, je steeds weer nieuwe ideeen en kritische blik tijdens alle fasen van het onderzoek hebben me enorm geholpen. Ik ben erg dankbaar voor de kans die je me hebt gegeven om zelf experimenten en onderzoek te doen, technieken op te zetten, te discussieren, te presenteren op congressen over de hele wereld, onderwijs te geven en manuscripten te schrijven, kortom om een goede wetenschapp(st)er te worden. Ondanks je protocollen uit Nijmegen en je voorliefde voor RNA ;-) kunnen we het toch goed vinden met elkaar, en ik hoop dat we nog lang 'good science' kunnen doen.

Beste Pieter, ook zonder jou was dit proefschrift niet geworden wat het nu is. Ik wil je bedanken voor je bijdrage, de geboden mogelijkheden, en het laten inzien dat klinische relevantie ook erg belangrijk is bij het onderzoek doen (ook al vind Tim van niet;-) ). Ik heb veel van je geleerd tijdens het opzetten en uitvoeren onze dierproef. Je enthousiasme en ambitie voor onderzoek werken aanstekelijk en ik hoop dat we nog lang samen onderzoek kunnen doen. $\mathrm{Nu}$ heb ik dadelijk eindelijk tijd om 'ons geheime inflammatieproefje' in te zetten.

I would also gratefully acknowledge the members of the assessment committee; Prof. Dr. Ir. L.H. Koole, Dr. F.A. van Nieuwenhoven, Prof. Dr. G.J.V.M. van Osch, Dr. M. Poeze and Prof. Dr. B. Zabel for their time and effort to critically judge this thesis. 
Don en Andy, ik wil jullie ook graag bedanken voor alle hulp bij de experimenten. Jullie staan altijd meteen voor iedereen klaar om te helpen, zorgen dat alles altijd op voorraad is en het lab geen grote chaos wordt ook al doen we daar zo ons best voor. Don, ik wil jou ook bedanken voor alle Don-koffietjes en momenten om te ventileren of gewoon lekker te kletsen. We gaan snel nog een keer eten en een ravanche doen met bowlen, deze keer heb ik geen pols-blessure... Andy, ik je ook bedanken voor zo strak opgeruimd houden van het lab en de $-80^{\circ} \mathrm{C}$ (stiekem werkt het toch best goed), het gewillig in mijn grapjes trappen en voor het regelen van de cup-a-soup carroussel! We kunnen nu al niet meer zonder.

Jim, Laura, Guus, Shennah, Alex, Maarten, Mandy, Ilona, Marielle, Michiel en Pieter ook jullie wil ik bedanken voor alle wetenschappelijke en minder wetenschappelijke discussies, jullie hulp en steun, voor het bijkletsen en de ontspannende momenten tijdens de cake-vande-week en bier-van-de-maand! Ook wil ik jullie bedanken voor alle uitstapjes tijdens de congressen, ze hebben al een hoop sterke verhalen opgeleverd. Who's next ?? ....

Chris en Liesbeth wil ik ook graag bedanken voor hun bijdrage aan dit proefschrift. Chris, als mede-brabander wil ik je bedanken voor de gezellige momenten tijdens alle congressen, voor je je kritische commentaar over mijn 'peppie-en-kokkie' onderzoekjes en het uitvinden 'de vork'- statement. Liesbeth, dankjewel dat je vaak een luisterend oor wilde zijn en voor alle gezellige momenten tijdens de afgelopen 4,5 jaar.

Ook wil ik hier Peter, Daan, Kathleen, Monique, Matheus, Fay en Olaf bedanken voor hun bijdrage aan de verschillende onderzoeken. Ik heb het erg leuk en leerzaam gevonden om jullie te begeleiden en hoop dat ik jullie heb kunnen helpen tijdens de stageperiodes.

Natuurlijk wil ik ook alle andere medewerkers van de afdeling Orthopedie bedanken, ik heb me altijd erg thuis gevoeld in deze groep.

Het Nfat5/osmolariteits onderzoek was niet mogelijk geweest dankzij de samenwerking met Dr. Holger Jahr en Drs. Anna van der Windt van het Erasmus MC in Rotterdam. Dankjewel voor deze mooie samenwerking en ik hoop dat we het in de toekomst voort kunnen zetten!

Ik ben ook zeer erkentelijk aan alle deelnemers en in het speicaal Jan-Willen Voncken van de WORK /Maastro/Bone meetings voor hun constructieve kritische blik en goede ideeen voor ons onderzoek.

Hier wil ik ook graag de research schools GROW en CAPHRI bedanken voor hun support tijdens dit promotie-traject. 
Voor de mooie tekening op de kaft wil ik graag Lindi Bronneberg bedanken, je hebt het geweldig gedaan!

Promotie-onderzoek is niet mogelijk als je niet af en je verhaal kwijt kan bij vriend(inn)en die hetzelfde doormaken. Caroline en Yvette, dankjewel voor jullie vriendschap en steun. Ook al moet het vaak via de email of telefoon, ik ben blij dat jullie er voor me zijn en dat ik niet de enige ben die dit doormaakt! Ik hoop dat we nog heel lang onze vriendschap kunnen doorzetten en gezellig door het hele land kunnen afspreken! Nina, wat heb ik ook veel steun gehad al onze korte koffie-pauzetjes en etentjes waarbij we onze verhalen even kwijt konden. Jammer dat 'onze' proefjes nog steeds niet van de grond zijn gekomen. Ik vind het super dat je als mijn paranimf naast me wil staan op mijn promotie! Ook wil ik hier Brenda bedanken, ik ben blij dat je als mijn 'oudste' vriendin er altijd voor me bent, ook al lukt het niet om zo vaak afspreken als we dat graag zouden willen.

Papa en Mama bedankt dat jullie er altijd voor me zijn. Jullie hebben altijd in me gelooft, me gestimuleerd om verder te leren en 'door te pakken' wanneer het nodig was. Zie hier het resultaat. Jan, Renske en Nick ook dankjewel voor jullie steun en alle momenten van onstpanning tijdens verjaardagen, winkel/kerstmarkt/musical/bierproef/ski -middagjes en avondjes enz. Die zijn erg belangrijk voor me. Mama en Renske moet ik hier ook bedanken voor het maken van de List of Abbriviations in dit proefschrift. Ook wil ik graag Laura, Peter, Ria en mijn toekomstige schoonfamilie bedanken voor hun steun en aanmoedigingen de afgelopen jaren. Daarnaast wil ik hier ook Frans noemen, waar ik zichtbaar en onzichtbaar veel steun aan heb gehad om te beginnen en door te gaan met universiteit en de promotie. Ik ben blij dat je mijn paranimf wilt zijn!

Lieve Toon, dit proefschrift had hier niet gelegen als jij er niet was geweest. Ik ben zo blij dat ik je heb gevonden, en dat bewijst maar weer eens dat je je liefde op de meest onverwachte plaatsen tegenkomt (het bureau schuinachter je). Je humor, relativeringsvermogen, wetenschappelijk inzicht en onvoorwaardelijke steun en liefde zijn onmisbaar voor me geweest de afgelopen jaren. Ik hoop dat we dit nog lang met elkaar mogen delen, ik hou van je ! 


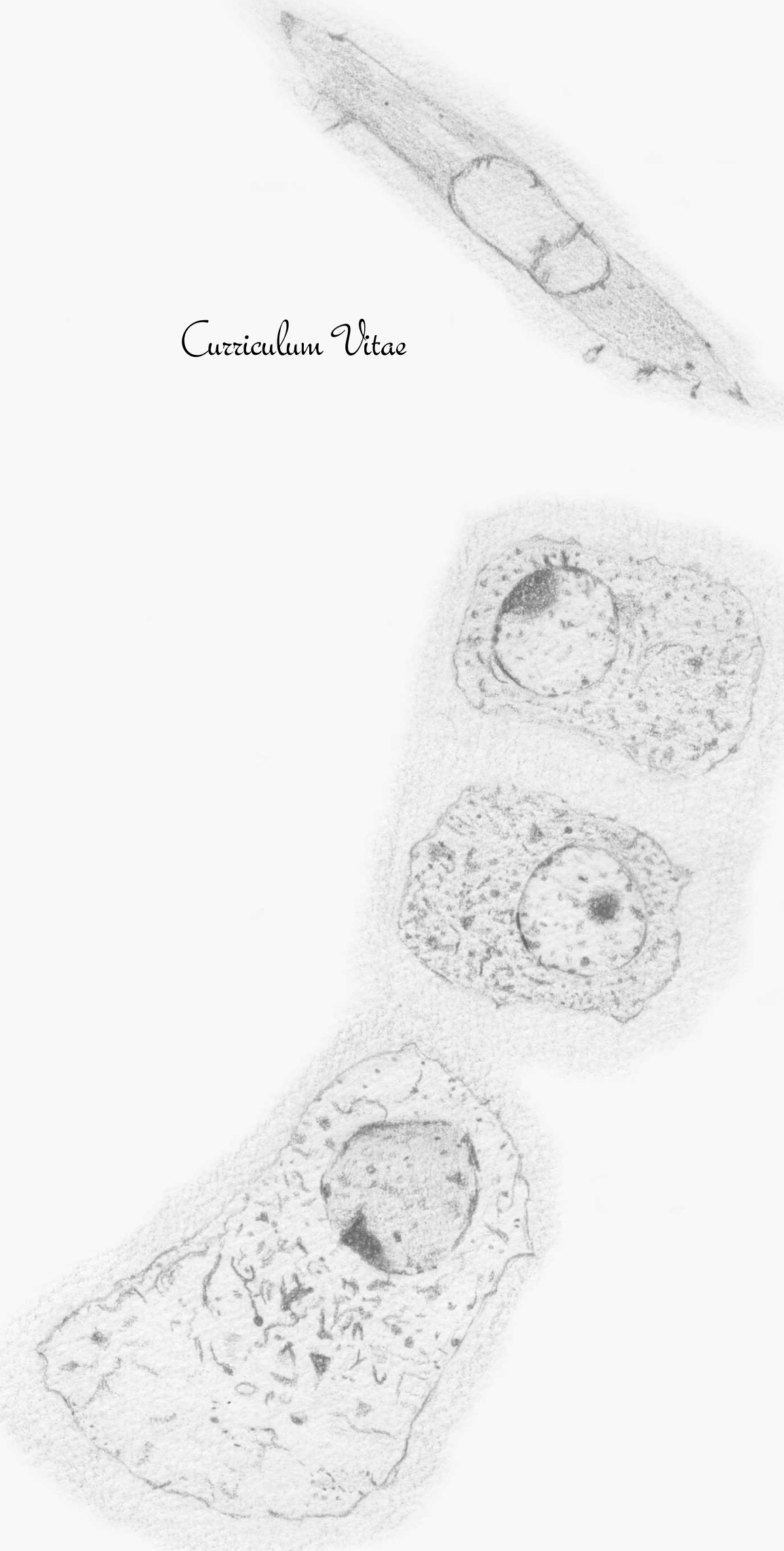



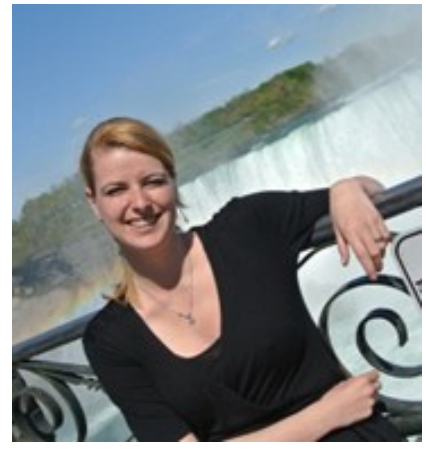

Marjolein Caron was born on May 2nd 1985 in Dongen, the Netherlands. In 2003 she received her diploma for preuniversity education (VWO) from the Cambreur College in Dongen. In September 2003 she started the study of General Health Sciences at Maastricht University, the Netherlands. Bioregulation and Health was her major program and Human Movement Sciences her minor program. She performed an internship at the department of Anatomy and Embryology at the Maastricht University where she studied the pathogenesis of spina bifida in murine Axd-embryo's. In August 2006 she received her Bachelor of Science degree with merit.

In September 2006, she started studying for a Master's degree in Clinical Molecular Science at the transnational University Limburg. During this study she performed an internship at the department of Nephrology, Technical University of München, Germany. She completed her master project at the departments of Molecular Genetics and Orthopaedic Surgery at the Maastricht University Medical Center which was focussed on hypoxia and epigenetics in chondrogenesis, and graduated in August of 2008.

In June of 2008 she got the opportunity to start her PhD-project on manipulation of the in situ incubator for cartilage and bone regeneration, described in this thesis, at the department of Orthopaedic Surgery at the Maastricht University Medical Center under supervision of Prof. Dr. L.W. van Rhijn, Dr. T.J.M. Welting and Dr. P.J. Emans. She presented parts of the work described in this thesis on various national and international conferences, and received the award for the best poster presentation at the 2008 NBTE (Nederlandse vereniging voor Biomaterialen en Tissue Engineering) annual meeting, the award for best oral presentation in basic science at the EORS (European Orthopaedic Research Society) $201220^{\text {th }}$ annual meeting and the NIRA award (New Investigator Recognition Award) on the ORS (Orthopaedic Research Society) 2013 annual meeting. She will continue her scientific career as a post-doc researcher at the department of Orthopaedic Surgery at the Maastricht University Medical Center. During this period she will focus on further studying molecular pathways in chondrogenic differentiation for the optimization of (progenitor) cell-based cartilage regenerative medicine. 



\section{List of Publications}
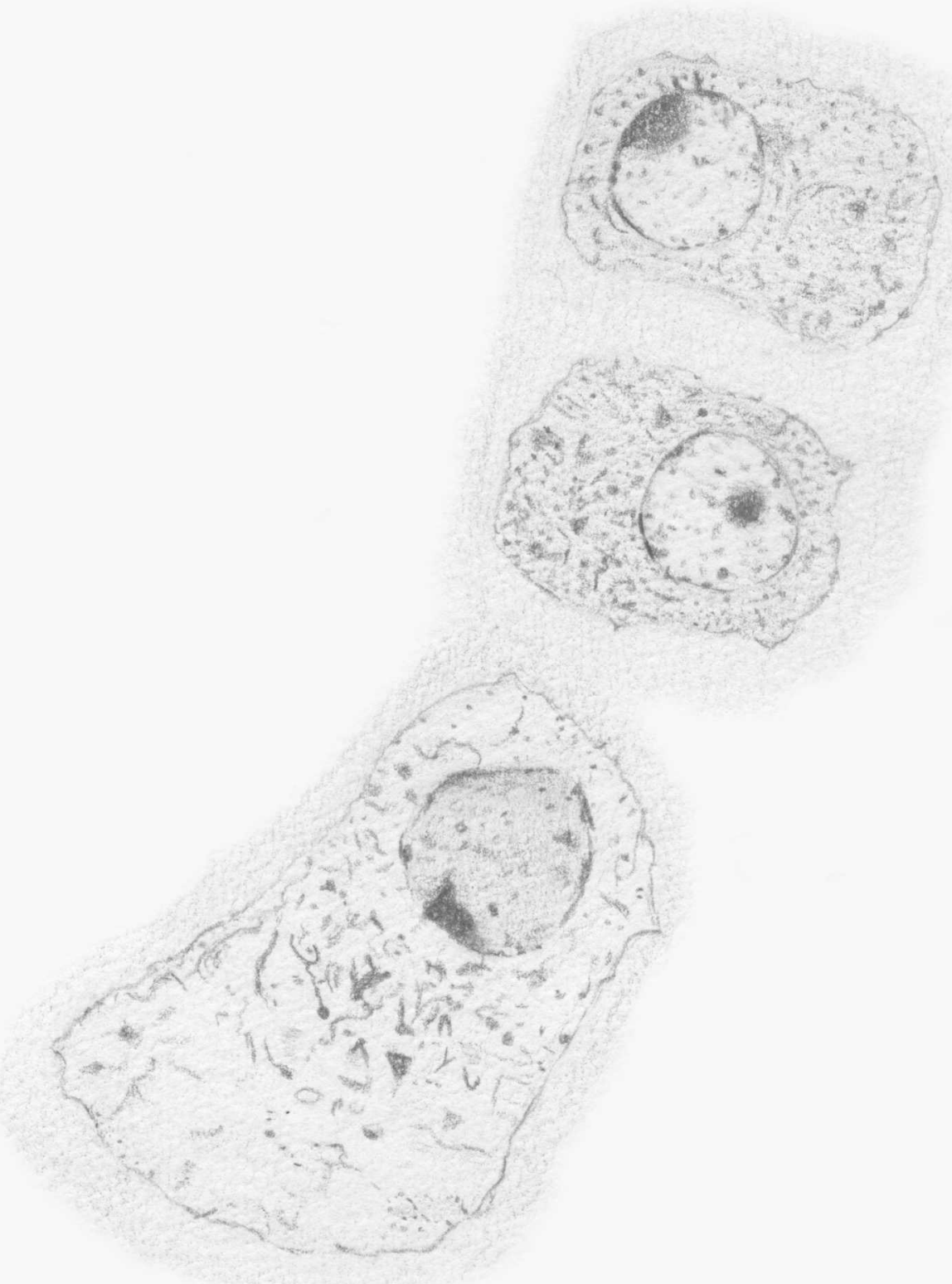

Peer reviewed pullications

Marjolein M.J. Caron, Pieter J. Emans, Don A. M. Surtel, Andy Cremers, Jan-Willem Voncken, Tim J.M. Welting and Lodewijk W. van Rhijn. (2012) Activation of NF-kB/p65 Facilitates Early Chondrogenic Differentiation during Endochondral Ossification. PLoS ONE 7 (3): e33467.

Marjolein M.J. Caron, Anna E. van der Windt, Pieter J. Emans, Lodewijk W. van Rhijn, Holger Jahr and Tim J.M. Welting. (2013) Tonicity Enhances the in vitro Chondrogenic Differentiation Capacity of Progenitor Cells. Bone 53(1): p.94-102

Marjolein M.J. Caron, Pieter J. Emans, Andy Cremers, Don A. M. Surtel, Marielle M.E. Coolsen, Lodewijk W. van Rhijn and Tim J.M. Welting. (2013) Differential Effects of BMP2 and BMP7 on Chondrogenic Differentiation of Progenitor Cells. Osteoarthritis and Cartilage (In press)

Tim J.M. Welting, Marjolein M.J. Caron, Pieter J. Emans, Maarten P.F. Janssen, Kathleen Sanen, Marielle M.E. Coolsen, Laura Voss, Don A.M. Surtel, Andy Cremers, JanWillem Voncken and Lodewijk W. van Rhijn. (2012) Inhibition of Cyclooxygenase-2 Impacts Chondrocyte Hypertrophic Differentiation During Endochondral Ossification. European Cells and Materials 22: p.420-436

Marjolein M.J. Caron, Pieter J. Emans, Don A.M. Surtel, Andy Cremers, Kathleen Sanen, Daan Ophelders, Lodewijk W. van Rhijn and Tim J.M. Welting. Differential Effects of Cyclooxygenase-1 and -2 Specific NSAIDs on Chondrogenic Differentiation. (In preparation)

Marjolein M.J. Caron, Pieter J. Emans, Marielle M.E. Coolsen, Laura Voss, Don A.M. Surtel, Andy Cremers, Lodewijk W. van Rhijn and Tim J. M. Welting. (2012) Redifferentiation of Dedifferentiated Human Articular Chondrocytes: Comparison of 2D and 3D Cultures. Osteoarthritis and Cartilage 20: p.1170-1178

Anna E. van der Windt, Esther Haak, Ruud H.J. Das, Nicole Kops, Tim J.M. Welting, Marjolein M.J. Caron, Niek P. van Til, Jan A.N. Verhaar, Harrie Weinans, Holger Jahr. (2010) Physiological Tonicity Improves Human Chondrogenic Marker Expression Through Nuclear Factor of Activated T-cells 5 in vitro. Arthritis research \& therapy 12 (3):R100

Marjolein M.J. Caron, Mandy M.F. Steinbusch, Kerstin Reichterer, Sandy Mattijssen, Don A.M. Surtel, E. Lausch, B. Zabel, Ger J.M. Pruijn, Lodewijk W. van Rhijn and Tim J.M. Welting. RNase MRP is regulated during skeletal development. (In preparation)

Frank Spaapen, Guus G.H. van den Akker, Marjolein M.J. Caron, Peggy Prickaerts, Vivian E.H. Dahlmans, Don A.M. Surtel, Celine Rofel, Yvette Paulis, Finja Schweizer, Tim J.M. Welting, Lars M. Eijssen and Jan-Willem Voncken. The immediate early gene EGR1 controls epigenetic reprogramming through Polycomb Group proteins during chondrogenesis. (Accepted for publication) 
Marcus Baumann, Marjolein M.J. Caron, Christoph Schmaderer, Christian Schulte, Ondreij Viklicky, Claus W. H. von Weyhern, Jens Lutz and Uwe Heemann. (2008) Renal $\mathrm{N}^{\varepsilon}$-Carboxymethyllysine Deposition After Kidney Transplantation. Transplantation 86:330 $-335$

Maarten P.F. Janssen, Marjolein M.J. Caron, Robert-jan R.B. van der Vorm, Bert van Rietbergen, Don A.M. Surtel, Andy Cremers, Lodewijk W. van Rhijn, Tim J.M. Welting and Pieter J. Emans. Systemic Inhibition of Cyclooxygenase-2 by Celecoxib Delays Endochondral Bone Fracture Healing. (In preparation)

\section{Revien}

Pieter J. Emans, Marjolein M.J. Caron, Lodewijk W. van Rhijn, Prasad V. Shastri and Tim J.M. Welting. (2011) Cartilage Tissue Engineering; Lessons Learned From Periosteum. J Tissue Sci Eng S2:002

Bookchapters

Pieter J. Emans, Marjolein M.J. Caron, Lodewijk W. van Rhijn and Tim J.M. Welting. (2012) Endochondral Bone Formation as Blueprint for Regenerative Medicine. Tissue Regeneration - From Basic Biology to Clinical Application. P.J. Davies (Ed.), InTech

Marjolein M.J. Caron, Tim J.M. Welting, Lodewijk W. van Rhijn, Pieter J. Emans. Targetting Inflammatory Processes for Optimization of Cartilage Homeostasis and Tissue Repair Techniques. ICRS: Developing Insights in Cartilage Repair. L. Peterson and P.J. Emans (Ed.), Springer-Verlag (Accepted for publication) 

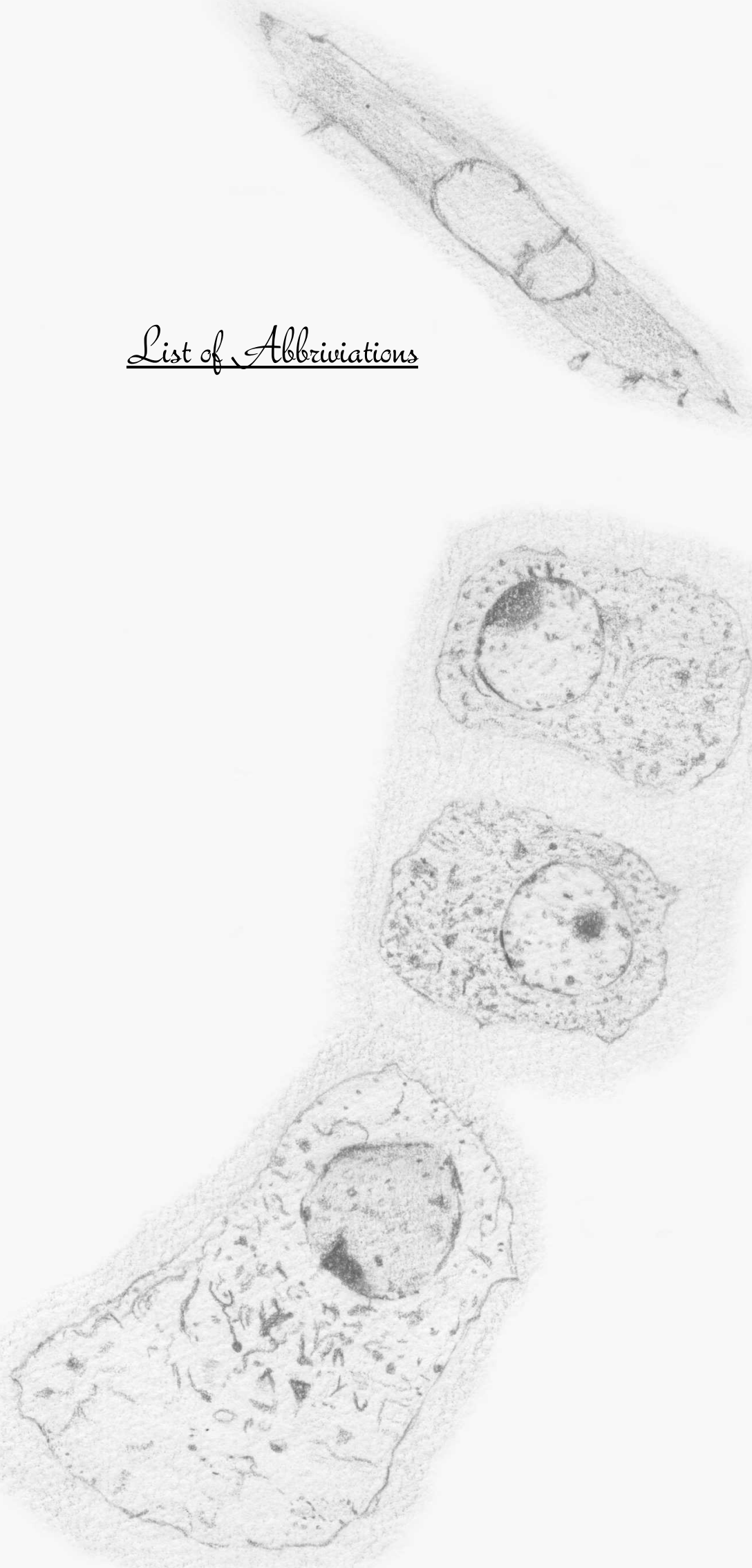



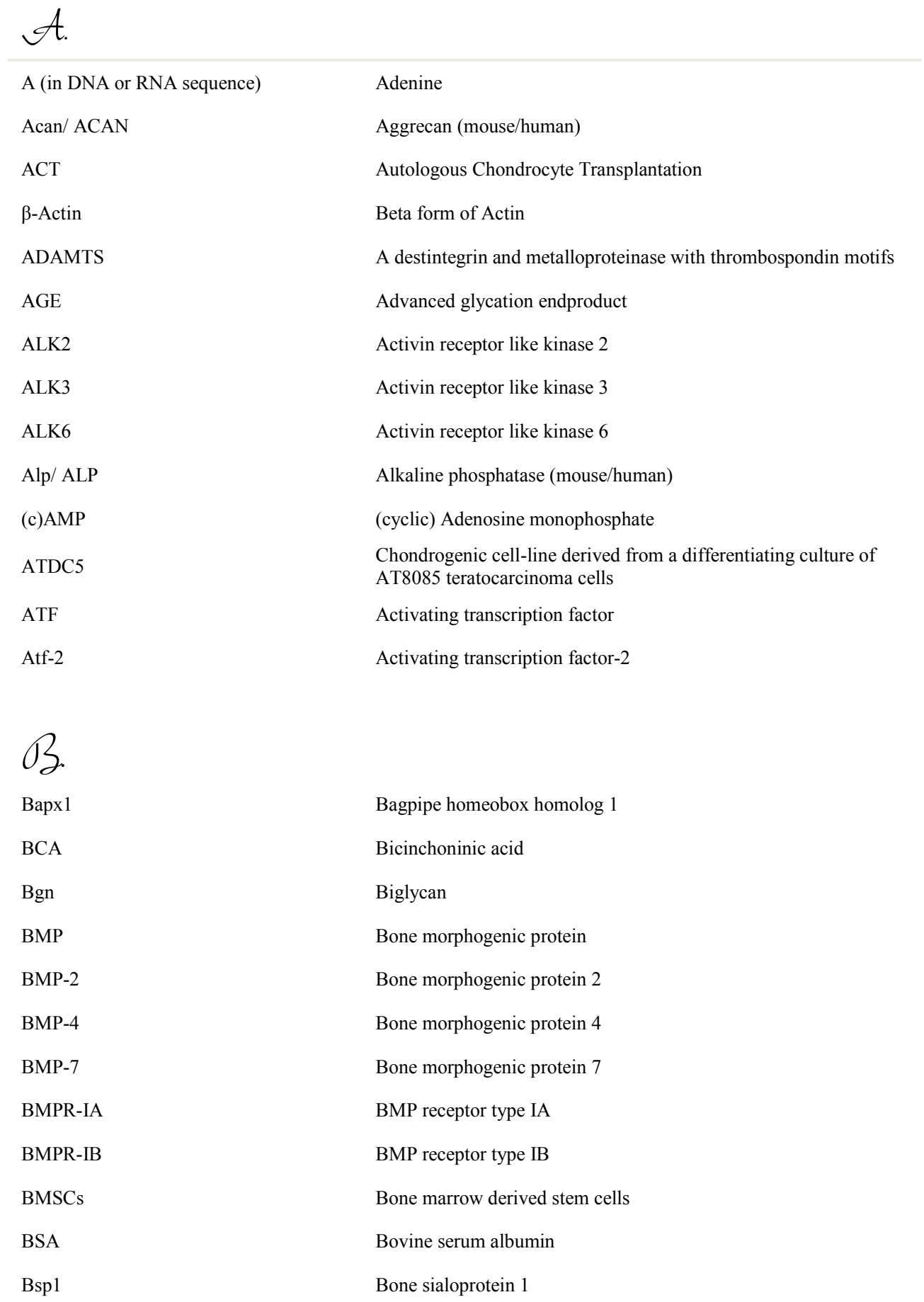




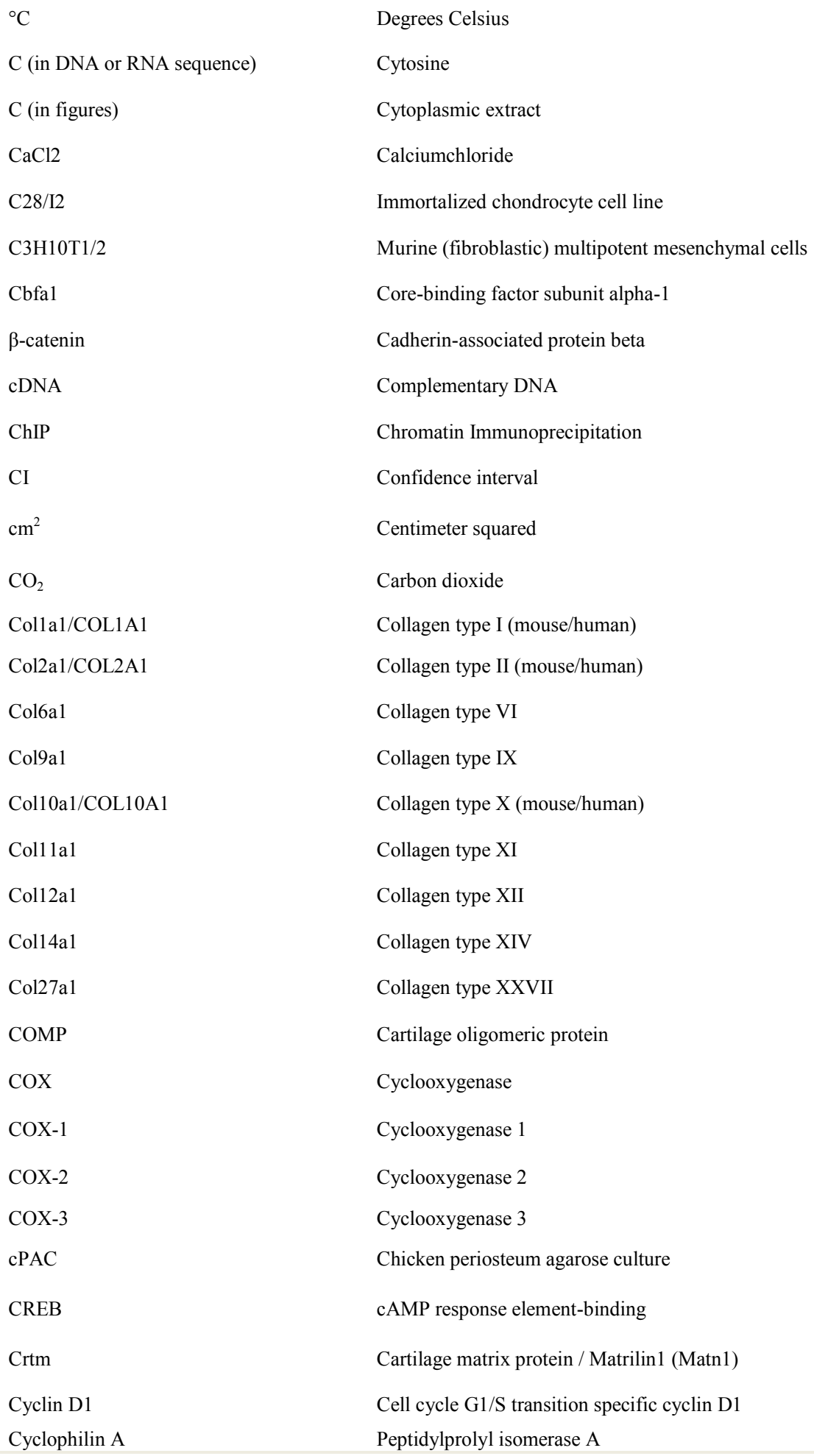




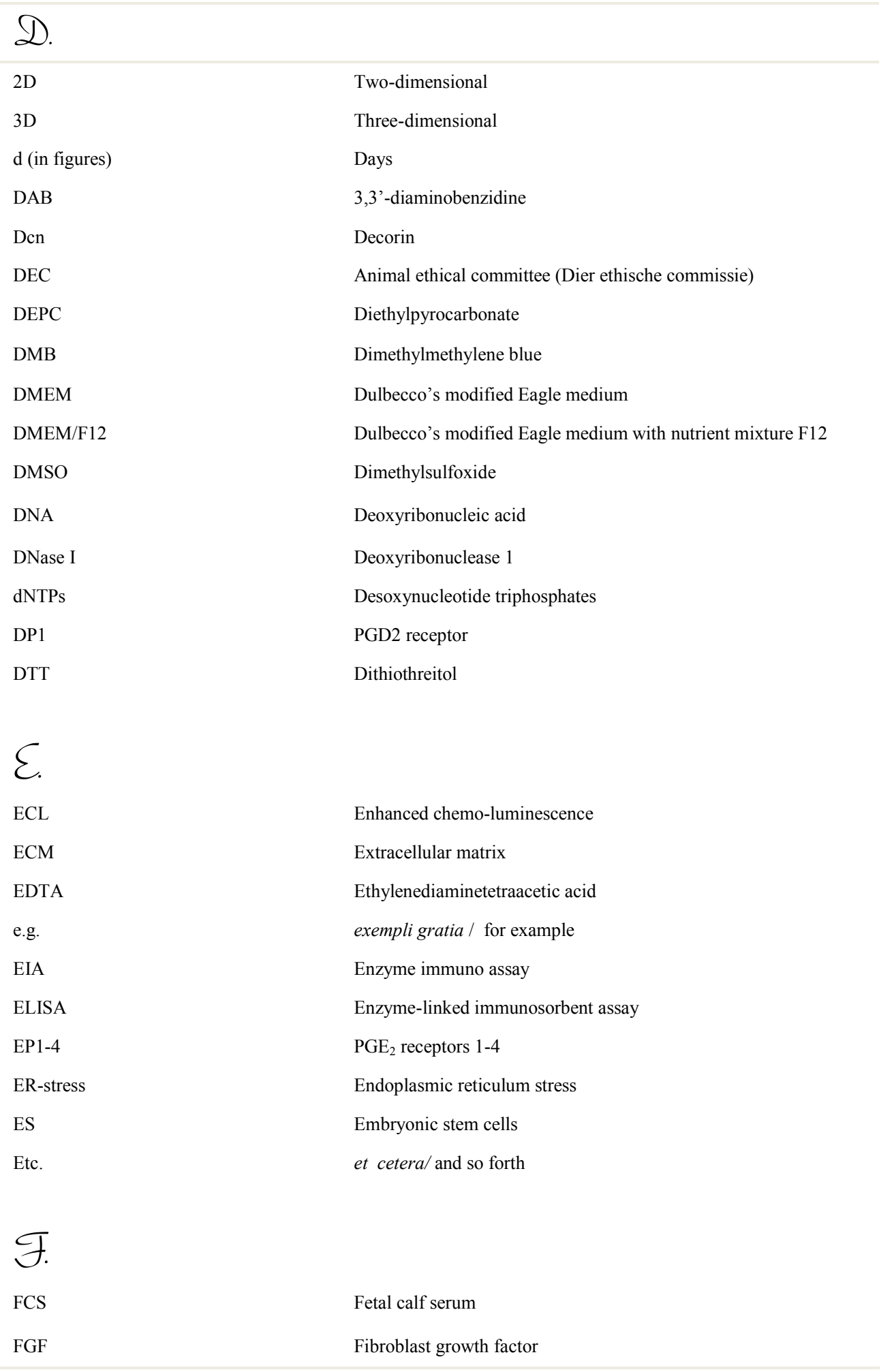




\section{FGF-2}

FGF-3

FGFR-3

Fmod

FP<smiles>C1CC[Te]C1</smiles>

G (in DNA or RNA sequence)

g

G-protein

GAG

GAPDH

GSK-3 $\beta$

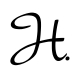

$\mathrm{h}$ (in figures)

H3

HAC

hBMSCs

$\mathrm{HCl}$

HEPES

HGF

Hif- $1 \alpha$

HMG

HRP

hrs

Hspg2

$\mathrm{HZ}$

9

ID

IGF
Fibroblast growth factor 2

Fibroblast growth factor 3

FGF receptor 3

Fibromodulin

$\mathrm{PGF}_{2 \alpha}$ receptor

Guanine

Gravitational force

Guanine nucleotide-binding protein

Glycosaminoglycan

Glyceraldehyde 3-phosphate dehydrogenase

Glycogen synthase kinase 3 beta

Hours

Histone 3

Human articular chondrocyte

Human bone marrow stem cells

Hydrochloric acid

4-(2-hydroxyethyl)-1-piperazineethanesulfonic acid

Hepatocyte growth factor

Hypoxia-inducible factor 1, alpha subunit

High mobility group

Horseradish peroxidase

Hours

Heparan sulphate proteoglycan 2 / perlecan

Hypertrophic zone of the growth plate

Identification

Insulin-like growth factor 


\begin{tabular}{|c|c|}
\hline IGF-1 & Insulin-like growth factor 1 \\
\hline IGFR & IGF receptor \\
\hline $\operatorname{IgG}$ & Immunoglobulin $\mathrm{G}$ \\
\hline IgG1 & Immunoglobulin G1 \\
\hline $\operatorname{IgG} 2 \mathrm{a}$ & Immunoglobulin G2a \\
\hline $\mathrm{IHC}$ & Immunohistochemistry \\
\hline Ihh & Indian hedgehog \\
\hline Il & Interleukin \\
\hline Il-1 & Interleukin 1 \\
\hline Il-1 $\beta$ & Interleukin 1 beta \\
\hline Il-6 / IL-6 & Interleukin 6 (mouse/human) \\
\hline iNOS & Inducible nitric oxide synthase \\
\hline IP & $\mathrm{PGI}_{2}$ receptor \\
\hline iPS & Induced pluripotent stem cells \\
\hline ITS & Insulin-transferrin-sodium selenite media supplement \\
\hline IVB & In vivo bioreactor \\
\hline \multicolumn{2}{|l|}{ K. } \\
\hline $\mathrm{KCl}$ & Potassium chloride \\
\hline $\mathrm{Kd}$ & Knockdown \\
\hline $\mathrm{kDa}$ & Kilodalton \\
\hline $\mathrm{KO}$ & Knockout \\
\hline \multicolumn{2}{|l|}{$\mathcal{L}}$. \\
\hline LPS & Lipopolysaccharide \\
\hline M & Molar \\
\hline MACT & Matrix-assisted chondrocyte transplantation \\
\hline MAPK & Mitogen-activated protein kinase \\
\hline $\mathrm{MCT}$ & $\begin{array}{l}\text { Mouse chondrocytes immortalized by SV } 40 \text { temperature-sensitive } \\
\text { large T antigen }\end{array}$ \\
\hline
\end{tabular}




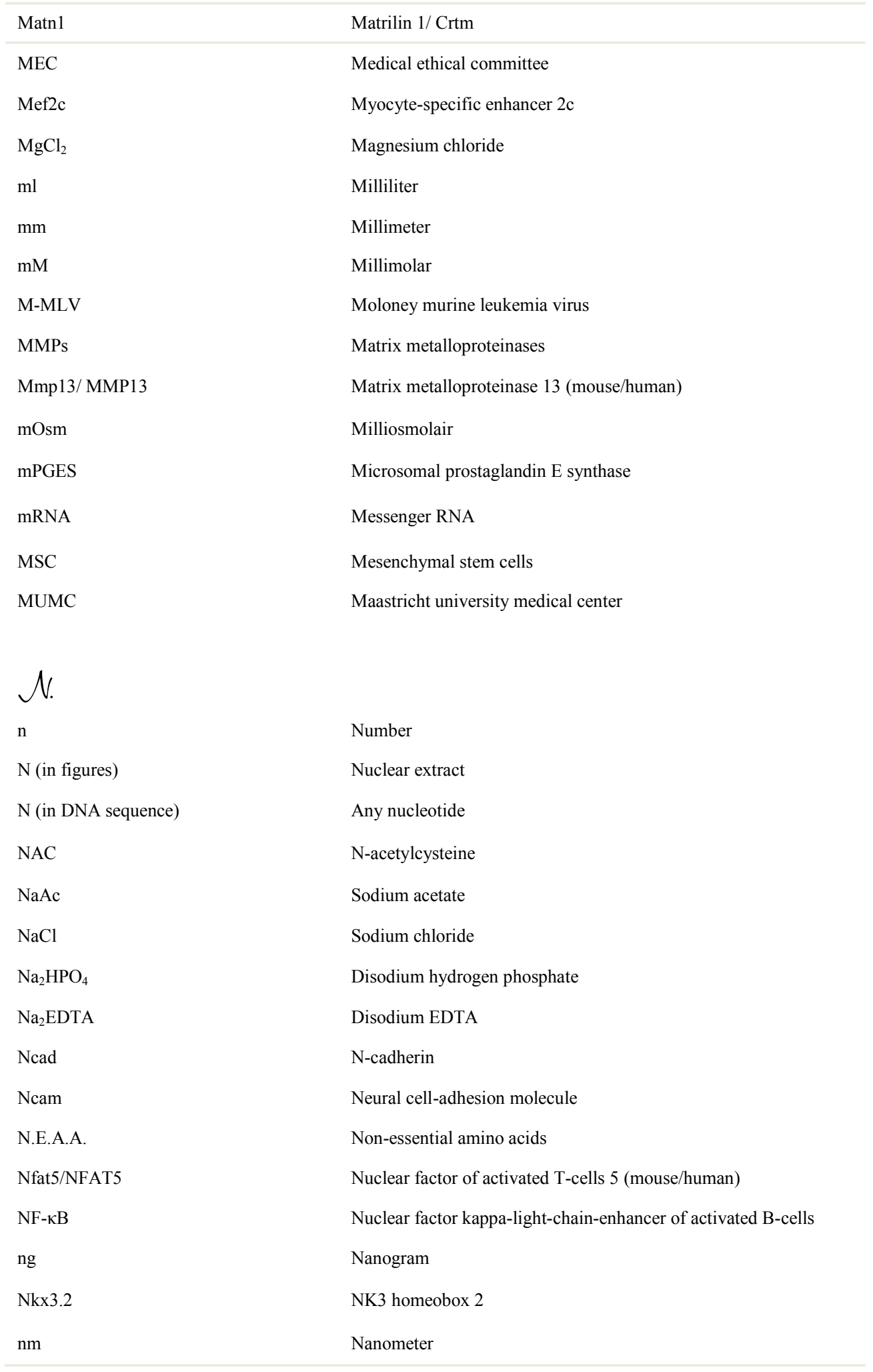




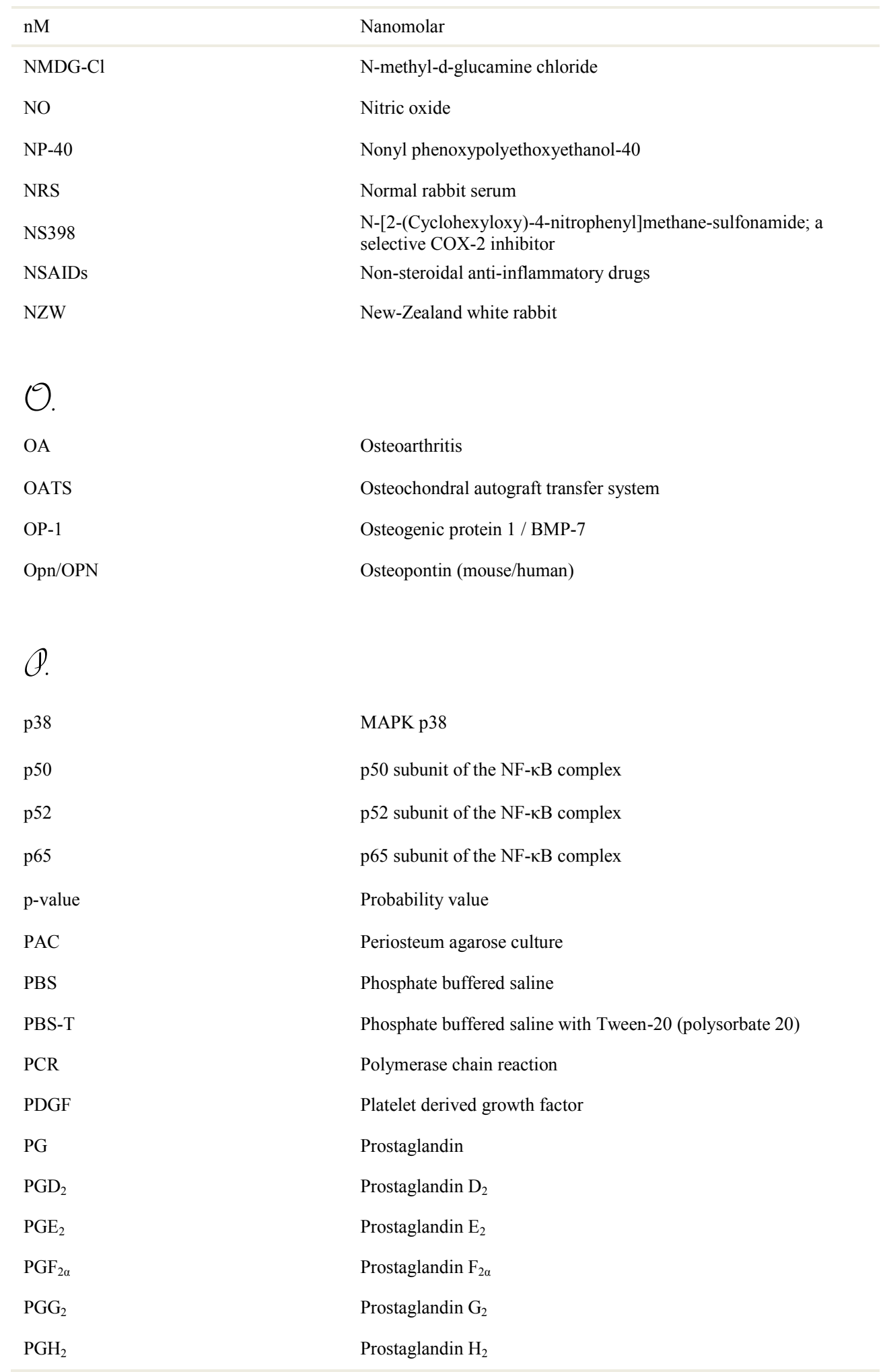




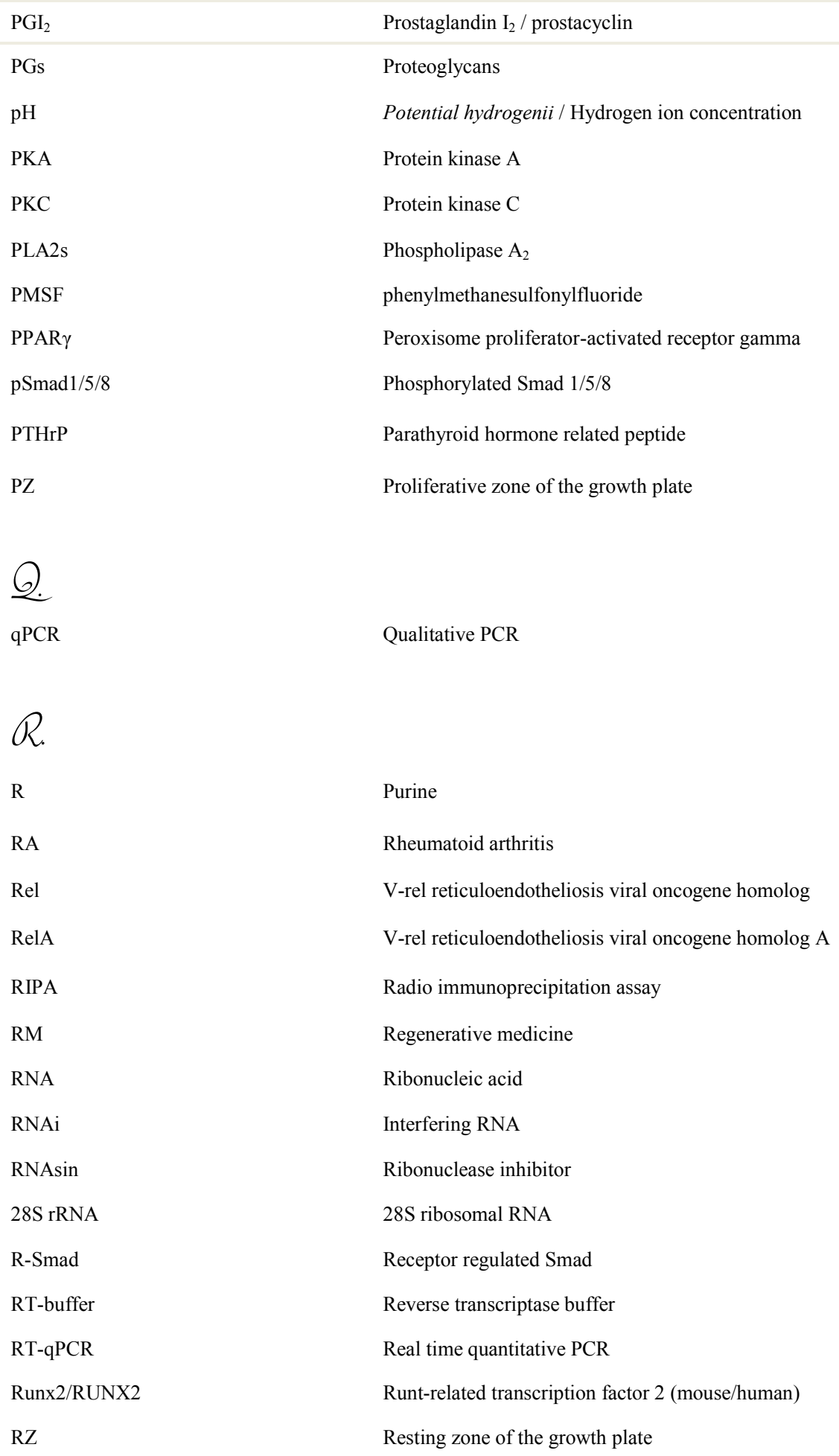




\begin{tabular}{|c|c|}
\hline S100A4 & S100 calcium binding protein 4 \\
\hline SC-560 & $\begin{array}{l}\text { 5-(4-chlorophenyl)-1-(4-methoxyphenyl)-3-(trifluoromethyl)-1H- } \\
\text { pyrazole, a selective COX-1 inhibitor }\end{array}$ \\
\hline Sdc3 & Syndecan 3 \\
\hline SDS & Sodium dodecyl sulphate \\
\hline SDS-PAGE & Sodium dodecyl sulphate - polyacrylamide gel electrophoresis \\
\hline s.e.m. & Standard error of mean \\
\hline SiRNA & Small-interfering RNA \\
\hline Slc5a3 & Solute carrier 5 (sodium/myoinositol cotransporter) member 3 \\
\hline Smad & $\begin{array}{l}\text { Homolog of Drosophila MAD (mothers against decapentaplegic) } \\
\text { and C. elegans SMA (small) }\end{array}$ \\
\hline Sox & SRY box \\
\hline L-Sox 5 & SRY (sex determining region $\mathrm{Y}$ ) box 5 , long form \\
\hline Sox6 & SRY (sex determining region Y) box 6 \\
\hline Sox9/SOX9 & SRY (sex determining region Y) box 9 (mouse/human) \\
\hline Sp1 & Specificity Protein 1 \\
\hline SRY & Sex determining region $\mathrm{Y}$ \\
\hline SW1353 & Human humerus chondrosarcoma cell line \\
\hline$T$. & \\
\hline $\mathrm{t}$ & Time point \\
\hline $\mathrm{T}$ (in DNA sequence) & Thymine \\
\hline $\mathrm{T}$ (in figures) & Total extract \\
\hline $\mathrm{T} / \mathrm{C} 28 \mathrm{a} 2$ & Immortalized human chondrocyte cell line \\
\hline $\mathrm{T} / \mathrm{C} 28 \mathrm{a} 4$ & Immortalized human chondrocyte cell line \\
\hline TBD & Triazabicyclodecene \\
\hline TBS-T & Tris buffered saline with Tween 20 (polysorbate 20) \\
\hline TBS-BSA & Tris buffered saline with Bovine serum albumin \\
\hline $\mathrm{TCF} / \mathrm{LEF}$ & Transcription factor/lymphoid enhancer-binding factor \\
\hline $\mathrm{TE}$ & Tissue Engineering \\
\hline TE buffer & Tris/EDTA buffer \\
\hline TGF- $\beta$ & Transforming growth factor beta \\
\hline
\end{tabular}


TGF- $\beta 1$

TGF- $\beta 2$

TGF- $\beta 3$

TGFR

\section{TLCK}

TLR-2

TLR-4

Tnc

$\mathrm{TNF} \alpha$

TNFR

TonEBP

$\alpha$-Tubulin

$\mathrm{TXA}_{2}$

$\mathrm{TXB}_{2}$

$q$.

$\mathrm{U}$ (in RNA sequence)

U

USAC

UV

$q$

Vcan

VEGF

VEGF-A

qe.

$\mathrm{w} / \mathrm{v}$

Wnt

$\mathscr{X}$
Transforming growth factor beta 1

Transforming growth factor beta 2

Transforming growth factor beta 3

TGF- $\beta$ receptor

$\mathrm{N}$-alpha-p-tosyl-1-lysinechloromethyl ketone hydrochloride, inhibitor of NF-kB

Toll-like receptor 2

Toll-like receptor 4

Tenascin C

Tumor necrosis factor alpha

$\mathrm{TNF} \alpha$ receptor

Tonicity responsive enhancer binding protein

Tubulin; alpha chain

Thomboxane $\mathrm{A}_{2}$

Thomboxane $\mathrm{B}_{2}$

Uracil

Units

Human chondrogenic osteosarcoma cell-line

Ultraviolet

Versican

Vascular endothelial growth factor

Vascular endothelial growth factor A

Weight/volume

Wingless-type MMTV integration-site family

$\mathrm{X}$ and $\mathrm{Y}$ chromosome 
y.

Y

2.

$\mathrm{ZnCl}_{2}$

Other.

$\mu \mathrm{g}$

$\mu 1$

$\mu \mathrm{m}$

$\mu \mathrm{M}$
Pyrimidine

Zinc chloride

Microgram

Microliter

Micrometer

Micromolar 


\section{GRO}

School for Oncology \&

Developmental Biology

\section{caphri}

School for Public Health and Primary Care
Reumafonds

Dutch Arthritis Foundation
Anna Fonds
Nederlands

Orthopedisch

Research en

Educatie

Fonds

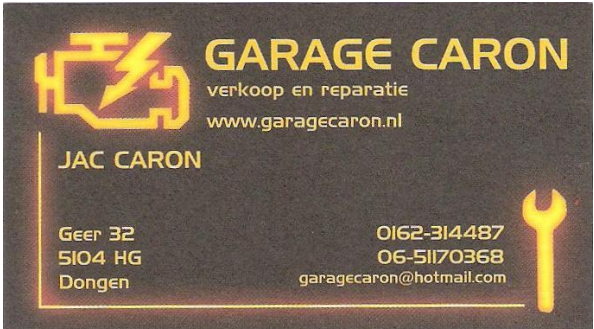



